OPEN

\title{
European Guideline Craniofacial Microsomia
}

\section{Ruben W. Renkema, MD and the ERN CRANIO Working Group on Craniofacial Microsomia}

Index

Summary

Chapter 1. Introduction 2393

1.1 Incentive for making the guideline 2393

1.2 Purpose of the guideline 2393

1.3 Scope of the guideline 2393

1.4 Relationship to other congenital facial malformations 2394

1.5 Intended users of the guideline 2394

1.6 About craniofacial microsomia 2394

1.7 European Reference Networks 2394

Chapter 2. Methodology 2394

2.1 Validity of the guideline 2394

2.2 General information 2394

2.3 Aim and target audience guideline 2394

2.3.1 Aim of the Guideline 2394

2.3.2 Target audience 2395

2.3.3 Patient population 2395

2.4 Steering group 2395

2.5 Conflicts of interest 2395

2.6 Patient perspectives 2396

2.7 Implementation 2397

2.8 Methods 2397

2.8.1 Bottleneck analysis 2397

2.8.2 Questions and outcomes 2397

2.8.3 Literature search and selection of literature 2397

2.8.4 Quality assessment of individual studies 2398

2.8.5 Summary of literature 2398

2.8.6 Quality of evidence 2398

2.8.7 Formulating conclusions 2398

2.8.8 Considerations 2398

2.8.9 Formulating recommendations 2400

2.8.10 Conditions (organisation of care) 2400

2.8.11 Knowledge gap 2400

2.8.12 Evaluation and authorisation phase 2400

Chapter 3. Diagnostic criteria for craniofacial microsomia 2400

3.1 Based on which criteria is a child or adult with craniofacial microsomia diagnosed? 2401

Chapter 4. Screening, monitoring and indication for treatment 2403

4.1 Breathing difficulties in craniofacial microsomia 2403

From the Department of Plastic and Reconstructive Surgery and Hand Surgery and Department of Maxillofacial Surgery, Dutch Craniofacial Center, Erasmus Medical Center, Rotterdam, The Netherlands.

Received March 29, 2020.

Accepted for publication April 21, 2020.

Address correspondence and reprint requests to Ruben W. Renkema, Dutch Craniofacial Center, Erasmus Medical Center, Rotterdam, The Netherlands; E-mail: r.renkema@erasmusmc.nl

Supplemental digital contents are available for this article. Direct URL citations appear in the printed text and are provided in the HTML and PDF versions of this article on the journal's Web site (www.jcraniofacialsurgery.com).

This is an open access article distributed under the terms of the Creative Commons Attribution-Non Commercial-No Derivatives License 4.0 (CCBY-NC-ND), where it is permissible to download and share the work provided it is properly cited. The work cannot be changed in any way or used commercially without permission from the journal.

Copyright $\odot 2020$ The Author(s). Published by Wolters Kluwer Health, Inc. on behalf of Mutaz B. Habal, MD.

ISSN: 1049-2275

DOI: $10.1097 /$ SCS.0000000000006691 
4.1.1 What is the type, prevalence and severity of breathing difficulties in craniofacial microsomia?

4.1.2 What is the policy for screening and monitoring of breathing difficulties (OSA) in patients with craniofacial microsomia?

4.1.3 What are the indications and policy for treatment of breathing difficulties (OSA) in patients with craniofacial microsomia?

4.2 Feeding difficulties in craniofacial microsomia

4.2.1 What is the type, prevalence and severity of feeding difficulties in craniofacial microsomia?

4.2.2 What is the policy for screening and monitoring of feeding difficulties in patients with craniofacial microsomia?

4.2.3 What are the indications and policy for treatment of feeding difficulties in patients with craniofacial microsomia?

4.3 Speech and Language difficulties in craniofacial microsomia

4.3.1 What is the type, prevalence and severity of speech difficulties in craniofacial microsomia?

4.3.2 What is the policy for screening and monitoring of speech and language difficulties in patients with craniofacial microsomia?

4.3.3 What are the indications and policy for treatment of speech difficulties in patients with craniofacial microsomia?

4.4 Hearing difficulties in craniofacial microsomia

4.4.1 What is the type, prevalence and severity of hearing difficulties in craniofacial microsomia?

4.4.2 What is the policy for screening and monitoring of hearing difficulties in patients with craniofacial microsomia?

4.4.3 What are the indications and policy for treatment of hearing difficulties in patients with craniofacial microsomia?

4.5 Eye anomalies in craniofacial microsomia

4.5.1 What is the type, prevalence and severity of eye anomalies in craniofacial microsomia?

4.5.2 What is the policy for screening and monitoring of eye anomalies in patients with craniofacial microsomia?

4.5.3 What are the indications and policy for treatment of eye anomalies in patients with craniofacial microsomia?

4.6 Dental deformities in craniofacial microsomia

4.6.1 What is the type, prevalence and severity of dental deformities in craniofacial microsomia?

2432

2432

4.6.2 What is the policy for screening and monitoring of dental deformities in patients with craniofacial microsomia?

2433

4.6.3 What are the indications and policy for treatment of dental deformities in patients with craniofacial microsomia?

4.7 Vertebral anomalies in craniofacial microsomia

4.7.1 What is the type, prevalence and severity of vertebral anomalies in craniofacial microsomia?

4.7.2 What is the policy for screening and monitoring of vertebral anomalies in patients with craniofacial microsomia?

4.7.3 What are the indications and policy for treatment of vertebral anomalies in patients with craniofacial microsomia?

4.8.1 What are the type, prevalence and severity of psychosocial difficulties in craniofacial microsomia?

2442

2443

4.8.2 What is the policy for the screening and monitoring of psychosocial difficulties in patients with craniofacial microsomia?

2445

4.8.3 What are the indications and policies for the treatment of psychosocial difficulties in patients with craniofacial microsomia?

5.1.1 What is the indication for surgical treatment of mandibular and maxillary deformity in patients with craniofacial microsomia?

5.1.2 What is the most optimal treatment modality and its timing for mandibular/maxillary deformity in patients with craniofacial

5.2.1 What is the indication for surgical treatment of facial nerve anomaly in patients with craniofacial microsomia?

5.2.2 What is the most optimal treatment modality for facial nerve anomaly in patients with craniofacial microsomia

5.3.1 What is the indication for surgical treatment of soft tissue deficiency in patients with craniofacial microsomia?

5.3.2 What is the most optimal treatment modality for soft tissue deficiency in patients with craniofacial microsomia 


\section{SUMMARY}

$\mathrm{T}^{\mathrm{h}}$ his guideline provides the following chapters:

Chapter 3 Diagnostic criteria for craniofacial microsomia Chapter 4 Screening, monitoring and indication for treatment

4.1 Breathing difficulties in craniofacial microsomia

4.2 Feeding difficulties in craniofacial microsomia

4.3 Speech difficulties in craniofacial microsomia

4.4 Hearing difficulties in craniofacial microsomia

4.5 Eye anomalies in craniofacial microsomia

4.6 Dental deformities in craniofacial microsomia

4.7 Vertebral anomalies in craniofacial microsomia

4.8 Psychosocial difficulties in craniofacial microsomia

Chapter 5 Surgical treatment of craniofacial microsomia

5.1 Mandible \& Maxilla

5.2 Facial nerve

5.3 Soft tissues

5.4 Microtia

Chapter 6 Organisation of care

The following recommendations were agreed on:

Chapter 3. Diagnostic criteria for craniofacial microsomia

3.1 On which criteria is a child or adult with craniofacial microsomia diagnosed?

\section{Terminology}

- It is advised to exclusively use the term craniofacial microsomia. Discard the use of other terms such as Goldenhar syndrome, hemifacial microsomia or auriculo-oculo-vertebral spectrum.

\section{Diagnostic criteria}

- It is advised to use the diagnostic criteria for craniofacial microsomia developed by the ICHOM Craniofacial Microsomia group.

CFM is defined by:

2 major criteria, or

1 major +1 minor criteria, or

$3+$ minor criteria

Major criteria

Mandibular hypoplasia

Microtia

Orbital / facial bone hypoplasia

Asymmetric facial movement

Minor criteria

Facial soft tissue deficiency

Pre-auricular tags

Macrostomia

Clefting

Epibulbar dermoids

Hemivertebrae

Chapter 4.1 Breathing difficulties in craniofacial microsomia

4.1.1 What is the type, prevalence and severity of breathing difficulties in craniofacial microsomia?

Since this question does not relate to interventions or diagnostics, only conclusions without any considerations, rationale or recommendations are provided.
4.1.2 What is the policy for screening and monitoring of breathing difficulties (OSA) in patients with craniofacial microsomia?

- All patients with craniofacial microsomia should be screened with a questionnaire biannually, at least up to the age of six, in the outpatient department for a clinical history of obstructive sleep apnoea.

- If there is a suspicion of obstructive sleep apnoea based on a questionnaire, a polysomnography (sleep study) has to be performed.

- All patients who have Pruzansky-Kaban IIb or III mandibles and/or are bilaterally affected have to undergo a polysomnography (sleep study) to screen for obstructive sleep apnoea in the first year of life.

4.1.3 What are the indications and policy for treatment of breathing difficulties (OSA) in patients with craniofacial microsomia?

- Treatment of children with craniofacial microsomia and obstructive sleep apnoea has to be discussed in a multidisciplinary team.

- Treatment of children with craniofacial microsomia and obstructive sleep apnoea depends on the age of the child, the severity of symptoms and the level of obstruction.

- In older children with mild to severe obstructive sleep apnoea, adenotonsillectomy (ATE) may be the treatment of first choice.

- In young infants and children with craniofacial microsomia and obstructive sleep apnoea non-surgical respiratory support has to be considered to treat obstructive sleep apnoea.

- In children with craniofacial microsomia and severe obstructive sleep apnoea a tracheostomy has to be considered at all ages. 


\section{Chapter 4.2 Feeding difficulties in craniofacial microsomia}

4.2.1 What is the type, prevalence and severity of feeding difficulties in craniofacial microsomia?

Since this question does not relate to interventions or diagnostics, only conclusions without any considerations, rationale or recommendations are provided.

4.2.2 What is the policy for screening and monitoring of feeding difficulties in patients with craniofacial microsomia?

- Children with craniofacial microsomia should be screened with a questionnaire biannually, at least up to the age of six, and monitored regularly for feeding difficulties by a paediatrician or multidisciplinary team.

- The WHO or national Growth Charts can be used to monitor growth and screen for feeding difficulties.

- A speech and language therapist should be involved in patients who require tube feeding.

4.2.3 What are the indications and policy for treatment of feeding difficulties in patients with craniofacial microsomia?

- Children with craniofacial microsomia with feeding difficulties should be treated by a multidisciplinary team.

- Feeding strategies are guided by the severity of feeding difficulties.

\section{Chapter 4.3 Speech difficulties in craniofacial microsomia}

4.3.1 What is the type, prevalence and severity of speech and language difficulties in craniofacial microsomia?

Since this question does not relate to interventions or diagnostics, only conclusions without any considerations, rationale or recommendations are provided.

4.3.2 What is the policy for screening and monitoring of speech and language difficulties in patients with craniofacial microsomia?

- Screen preverbal communication and babbling skills at the age of nine months to decide if intervention is warranted.

- Evaluate receptive and expressive language skills at the age of two years and biannually until the age of eight years in all patients with craniofacial microsomia. Those identified with difficulties should be referred to their community speech and language therapist service for ongoing intervention.

- Oral-facial evaluation of structure and function is recommended at each screening consultation to examine any impact of asymmetry on speech production. This should include examination of facial symmetry, lips, dental occlusion and intra-oral examination of tongue movement, dentition, hard palate and soft palate movement on sustained 'ah' vowel.

- Screen patients with tracheostomy for speaking valve suitability or an augmentative and alternative communication system.

- Social communication skills should be monitored in tangent with all of the afore-mentioned communication skills.

- Children with craniofacial microsomia and associated cleft palate should be screened annually from $2-5$ years by the Cleft-Craniofacial speech and language therapist and should follow the local Cleft Palate Protocol. Velopharyngeal dysfunction should be assessed from the age of two years or when verbal output has emerged.

- Children with craniofacial microsomia without a cleft palate should also be screened at the age of two years to examine for potential risk of velopharyngeal dysfunction related to their asymmetrical structure. If velopharyngeal dysfunction is identified, these children should follow the same pathway as children with a cleft palate.

4.3.3 What are the indications and policy for treatment of speech and language difficulties in patients with craniofacial microsomia?

- Recommend early language stimulation for delayed babble onset from nine months.

- Facilitate receptive and expressive language development using a range of behavioural techniques such as modelling, imitation, repetition and extension.

- Patients with cleft speech characteristics should have articulation therapy when identified. Direct therapy using an articulation approach is recommended from age three onwards

- Monitor patients with tracheostomy and speaking valve use on a regular base.

- Introduce low or high tech augmentative and alternative communication systems to children who are non-verbal or whose speech is unintelligible. These include gestures, signing, symbols, word boards, communication boards and books, as well as Voice Output Communication Aids (VOCAs). A low-tech system such as signing can be introduced from one.

- Intervention for social communication difficulties is recommended; e.g. development of non-verbal communication skills (e.g. eye contact, turn-taking); conversational skills, recognitions of emotions and emotional regulation.

Chapter 4.4 Hearing difficulties in craniofacial microsomia

4.4.1 What is the type, prevalence and severity of hearing difficulties in craniofacial microsomia?

Since this question does not relate to interventions or diagnostics, only conclusions without any considerations, rationale or recommendations are provided.

4.4.2 What is the policy for screening and monitoring of hearing difficulties in patients with craniofacial microsomia? 
- Perform neonatal hearing test in all new-borns with craniofacial microsomia. If indicated, complete audiological evaluation in an experienced audiology centre should be performed before the age of three months to ensure timely treatment.

- Re-evaluate hearing tests in patients with craniofacial microsomia by the age of 24-30 months.

- Regularly perform otoscopy and audiometry in patients with craniofacial microsomia including microtia and/or cleft palate by the ENT doctor/otolaryngologist.

- Audiologic intervention should be initiated before the age of six months in patients with congenital hearing loss.

4.4.3 What are the indications and policy for treatment of hearing difficulties in patients with craniofacial microsomia?

- Treat moderate to severe hearing loss, either with nonsurgical or surgical options.

- Coordinate surgical approach and timing in a multidisciplinary team regarding hearing augmentation and other surgical procedures including ear reconstruction and mandibular surgeries.

Chapter 4.5 Eye anomalies in craniofacial microsomia

4.5.1 What is the type, prevalence and severity of eye anomalies in craniofacial microsomia?

Since this question does not relate to interventions or diagnostics, only conclusions without any considerations, rationale or recommendations are provided.

4.5.2 What is the policy for screening and monitoring of eye anomalies in patients with craniofacial microsomia?

- All patients with craniofacial microsomia should be screened at least once during the visual development (before the age of five) by an orthoptist and ophthalmologist. Depending on the results, follow-up visits need to be scheduled on a regular basis.

4.5.3 What are the indications and policy for treatment of eye anomalies in patients with craniofacial microsomia?

- Children with ocular disturbances need to be evaluated by a specialised orthoptist and ophthalmologist during the visual development (before the age of five).

- Optimal spectacle correction should be provided in case of a refractive error.

- Amblyopia should be treated before the age of six.

- When surgery is considered this has to be discussed in a multidisciplinary team, carefully evaluating the harms and the benefits, especially in the case of young children in whom vision is still developing.

- Ultrasound imaging of the ocular dermoid needs to be conducted if extension posteriorly and into the orbit is suspected.
Chapter 4.6 Dental deformities in craniofacial microsomia

4.6.1 What is the type, prevalence and severity of dental deformities in craniofacial microsomia?

Since this question does not relate to interventions or diagnostics, only conclusions without any considerations, rationale or recommendations are provided.

4.6.2 What is the policy for screening and monitoring of dental deformities in patients with craniofacial microsomia?

- Patients with craniofacial microsomia should have routine dental care.

- Patients with craniofacial microsomia should be seen from age five by an orthodontist within a multidisciplinary team to diagnose dental deformities.

- Perform screening for dental deformities by intra-oral inspection and standard dental records.

- Take orthodontic records in a structured schedule, at 6, $9,12,15$ and 18 years of age.

4.6.3 What are the indications and policy for treatment of dental deformities in patients with craniofacial microsomia?

- Dentofacial orthopaedic treatment can be considered appropriate in very mild craniofacial microsomia cases. In severe craniofacial microsomia patients, current evidence does not promote activator treatment.

- Orthodontic treatment should be discussed and coordinated in a multidisciplinary team depending on the decision to conduct orthognathic surgery or not.

\section{Chapter 4.7 Vertebral anomalies in craniofacial} microsomia

4.7.1 What is the type, prevalence and severity of vertebral anomalies in craniofacial microsomia?

Since this question does not relate to interventions or diagnostics, only conclusions without any considerations, rationale or recommendations are provided.

4.7.2 What is the policy for screening and monitoring of vertebral anomalies in patients with craniofacial microsomia?

- Screening questions and clinical examinations related to neck/back symptoms should be undertaken at initial consultation and as part of pre-operative workup.

- All patients with craniofacial microsomia who have neurologic symptoms (e.g., paraesthesia, numbness, or weakness) or neck pain suggestive of neuronal injury should be evaluated as soon as possible by a (paediatric) neurologist.

- Patients should be referred appropriately and attention to the cervical spine should be payed when patients are undergoing general anaesthesia.

4.7.3 What are the indications and policy for treatment of vertebral anomalies in patients with craniofacial microsomia? 
- Surgical fusion and/or bracing in patients with vertebral anomalies may be necessary to obtain spinal stability and to prevent secondary injury of the spinal structures.

- A multidisciplinary approach in treatment and timing is warranted to optimise outcomes for these patients.

Chapter 4.8 Psychosocial difficulties in craniofacial microsomia

4.8.1 What is the type, prevalence and severity of psychosocial difficulties in craniofacial microsomia?

Since this question does not relate to interventions or diagnostics, only conclusions without any considerations, rationale or recommendations are provided.

4.8.2 What is the policy for screening and monitoring of psychosocial difficulties in patients with craniofacial microsomia?

- All craniofacial microsomia patients should have access to a clinical psychology service with appropriate professional expertise and knowledge of craniofacial microsomia.

- Time points for reviews and screening should observe key life transitions such as birth, starting school, transition to secondary school, etc.

- To measure psychosocial wellbeing and family stress, validated self-reported psychological outcome measures should be obtained from to all craniofacial microsomia patients as a matter of routine to screen for the presence of behavioural, emotional, social and/or learning difficulties. This includes the CleftQ, CFEQ, YP-CORE, HADS and Distress Thermometer for Parents and should be performed at age 2, 5, 8 and 22. Elevated scores should alert clinicians to the potential need for further assessment or support. Standardised measures should assess levels of emotional distress as well as evaluate difficulties related to visible differences.

4.8.3 What are the indications and policy for treatment of psychosocial difficulties in patients with craniofacial microsomia?

\section{Parental adjustment and support}

- Parents of newly diagnosed children with craniofacial microsomia should have access to a specialist clinical psychology service with expertise and knowledge of the condition.

- Information on support groups and organisations should be provided, both at initial contact and at regular review.

\section{Behavioural and/or learning difficulties}

- When appropriate, clinicians should liaise with local services and schools to discuss the child's support needs.

- Cognitive assessment may be offered if warranted

\section{Coping with visible difference}

- Patients with craniofacial microsomia should have access to specialist psychological support, particularly those who are presenting with low self-esteem, depression/low mood, anxiety, appearance- or treatment-related concerns, including adjustment difficulties or trauma as a result of surgical/medical interventions.

- Clinicians with appropriate professional expertise in craniofacial microsomia should consider liaising with local schools to offer advice on how to support children who have visible differences.

- Information about support groups and organisations should be provided.

- Psychological input is required pre- and post- facial surgery to monitor expectation and acceptance.

- The psychologist is part of the coordinated care in the multidisciplinary team. See recommendations in Chapter 6.

\section{Chapter 5.1 Mandible \& Maxilla}

5.1.1 What is the indication for surgical treatment of mandibular and maxillary deformity in patients with craniofacial microsomia?

- Consider surgical management (tracheostomy, adenotonsillectomy, mandibular and/or maxillary surgery) in patients with craniofacial microsomia for the treatment of breathing problems if non-surgical therapy fails or to end non-surgical therapy.

- Inform patients and parents about of the uncertainty of respiratory outcomes following mandibular and/or maxillary surgery for OSA in patients with CFM.

- If surgical treatment of the mandibular/maxillary deformity in patients with craniofacial microsomia is indicated to prevent or treat psychosocial problems, it is important to inform the patient about the potential benefits and harms and to ensure that the patients/ parents have a realistic view of what can be expected.

- It is advised to integrate the (surgical) treatment of the mandibular/maxillary deformity in patients with craniofacial microsomia in the planning of other surgeries, especially for those that affect facial symmetry, palsy, soft tissue augmentation and treatment of atresia or microtia.

5.1.2 What is the most optimal treatment modality and its timing for mandibular/maxillary deformity in craniofacial microsomia regarding severity, breathing problems, occlusal problems and aesthetics?

\section{Obstructive sleep apnoea}

- Start with non-surgical treatment for the management (e.g. oxygen, CPAP) of mild-moderate OSA in infants with craniofacial microsomia. See Chapter 4.1 Breathing difficulties for recommendations.

- Perform a tracheostomy or mandibular distraction osteogenesis in infants with mandibular hypoplasia 
and severe OSA who do not respond to nonsurgical treatment.

- If the aim of surgical treatment is to end non-surgical treatment (e.g. CPAP), perform elective mandibular distraction osteogenesis.

- Mandibular reconstruction with costochondral bone grafts should be performed after the age of six.

\section{Occlusal problems}

- For patients with craniofacial microsomia and severe occlusal problems, perform mandibular distraction osteogenesis in mixed dentition phase.

- A combined orthodontic and orthognathic surgery plan is mandatory to achieve and optimise stable longterm outcomes.

- Perform secondary orthognathic surgery to correct occlusion at skeletal maturity.

\section{Aesthetic problems}

- Postpone surgical correction of the mandibular/maxillary deformity for aesthetic reasons in patients with craniofacial microsomia until skeletal maturity.

- The implications of early surgery (i.e. repeat surgery) for psychosocial reasons should be discussed within the multidisciplinary team and with patient and caregivers.

- Psychological input is required pre- and post-operatively to monitor expectation and acceptance.

\section{$3 D$ planning}

- Use 3D planning to optimise surgical outcome of mandibular and maxillary surgery in patients with CFM.

\section{Chapter 5.2 Facial nerve}

5.2.1 What is the indication for surgical treatment of facial nerve anomaly in patients with craniofacial microsomia?

\section{Indications for treatment}

- Provide all patients with craniofacial microsomia with psychosocial support.

- Refer all craniofacial microsomia patients with lagophthalmos to an ophthalmologist.

- Surgical treatment of the upper or lower eyelids should be considered in patients with craniofacial microsomia and loss of function of the upper facial nerve branches.

- Coordinate the timing of facial reanimation surgery in patients with craniofacial microsomia in the planning of other major surgeries.

- Facial movement should by assessed with the CleftQ Appearance at age 8, 12, and 22 .
5.2.2 What is the most optimal treatment modality for facial nerve anomaly in patients with craniofacial microsomia related to functional deficits and aesthetics?

Eye

- Correct lagophthalmos due to facial palsy in patients with craniofacial microsomia with placement of a gold weight or platinum chain, muscle transfers and/or tendon slings, or cross-facial nerve grafting.

- Tarsorrhaphy as a treatment for lagophthalmos in patient with craniofacial microsomia is discouraged.

Oral

- Start with the injection of botulinum toxin in the nonaffected depressor labii inferioris muscle if therapy is indicated in patients with craniofacial microsomia and asymmetrical lip depression due to facial palsy.

- Consider myomectomy of the non-affected depressor labii inferioris muscle if the outcome of treatment with botulinum toxin injections are satisfactory.

- Consider the use of dynamic techniques such as digastric muscle transfers if the outcome of treatment with botulinum toxin injections are not satisfactory.

- Perform imaging of the digastric muscle prior to surgical muscle transfer due to the high prevalence of agenesis of the anterior belly of the digastric muscle.

\section{Aesthetics}

- Psychological input is required pre- and post-operatively to monitor expectation and acceptance.

- Strive for spontaneous facial animations by using a cross-facial nerve graft with a free flap.

- Consider functional muscle transfer from the age of four onwards.

- Reserve the use of the masseteric nerve to innervate the free muscle transfer for patients in whom cross-facial nerve grafting is not favourable, in bilateral cases, or as a babysit procedure.

- Consider the use of regional muscle transfers to achieve facial animation in patients with craniofacial microsomia and facial palsy if cross-facial nerve grafting with free muscle transfers is not preferred.

- A facial physical therapist is part of the multidisciplinary team.

- Collect clinician- and patient-reported outcome measures pre- and posttreatment.

\section{Chapter 5.3 Soft tissues}

5.3.1 What is the indication for surgical treatment of soft tissue deficiency in patients with craniofacial microsomia?

- The indication for surgical treatment of soft tissue deficiency in patients with craniofacial microsomia is mainly aesthetic. Inform the patient about the potential 
benefits and harms to ensure that the patient has a realistic view of what can be expected.

- Patients' difficulties with facial form/asymmetry should be assessed with the CleftQ Appearance at age 8, 12, and 22 .

5.3.2 What is the most optimal treatment modality for soft tissue deficiency in patients with craniofacial microsomia related to severity and its timing?

- Psychological input is required pre- and post-operatively to monitor expectations and acceptance.

- Reconstruct soft tissue deficiencies in patients with craniofacial microsomia with fat grafting from childhood.

- Free tissue transfer is only considered in patients with a very severe soft tissue deficiency.

- Alloplastic implants to correct soft tissue deficiency in patients with craniofacial microsomia are ideally performed at skeletal maturity.

- The use of pedicled flaps for correction of soft tissue deficiency in patients with craniofacial microsomia is strongly discouraged.

- Coordinate the timing of surgical treatment of soft tissue deficiency in patients with craniofacial microsomia with the planning of other surgeries, especially for surgeries that affect facial symmetry such as mandibular surgeries or placement of facial implants.

\section{Chapter 5.4 Microtia}

5.4.1 What is the indication for surgical treatment of ear deformity in patients with craniofacial microsomia?

- The indication for auricular reconstruction in patients with craniofacial microsomia is aesthetic and psychosocial. Inform the patient about the potential benefits and harms to ensure that the patient has a realistic view of what can be expected.

- Provide all patients with craniofacial microsomia with psychosocial support.

- Use the PROM Ear-Q pre- and postoperatively to assess benefit of treatment.

5.4.2 What is the most optimal treatment modality for ear deformity in patients with craniofacial microsomia related to its timing?

- Patients should be treated within a multidisciplinary team setting.

- Discuss the advantages and disadvantages of the various treatment modalities with the patient and base the choice for treatment on patients' preferences.

- Psychological input is required pre- and postoperatively to monitor expectation and acceptance.

- Ear reconstruction with rib grafts is the first choice of treatment.

- Perform ear reconstruction with rib from the age of eight onwards.
- Treatment before the age of eight is not recommended, but if chosen, use external silicone prosthesis attached with adhesives.

- If chosen, place polyethylene implants (Medpore) from the age of six onwards.

- Osseointegrated implants are an option for salvage procedures.

- Outcome measures should be obtained pre- and postoperatively with all techniques and interventions.

Chapter 6 What are the minimal care standards to treat patients with craniofacial microsomia and how should outcomes of care be monitored?

\section{Information}

- The multidisciplinary team should provide information regarding the condition and treatment options based on the present craniofacial microsomia guideline in their own language.

\section{Referral}

- Patients should be referred to the multidisciplinary craniofacial microsomia team in a timely manner.

\section{Collaboration}

- Care for patients with craniofacial microsomia should be delivered by the multidisciplinary team.

- The clinical pathway based on this guideline should be followed.

\section{Communication}

- Communication between and within teams (also in different hospitals) should be initiated to facilitate the best possible treatment. A contact person in each centre - a care coordinator - clarify and facilitate communication between different institutions and within her/his own institution.

\section{Conditions}

- A craniofacial centre has the following care providers:

- Maxillofacial surgeon

- Plastic surgeon

- ENT/audiology

- Psychology

- Orthodontics

- Ophthalmologist

- Paediatric anaesthesiologists 
○

○

$\circ$

(1)

- Neurosurgeon and/or orthopaedic surgeon for spinal anomalies

- Paediatric radiologist

- Social worker

- Speech therapists

- Pedagogical worker

- (Facial) physical therapist

Prosthetist

- Respiratory team

A craniofacial centre has access to the following care - facilities:

- (3D)photography, roentgen, CT, MRI, 3D-planning facility

- Paediatric ICU

- Sleep study facility

- Audiological evaluation

- Dental lab

\section{Transitional care}

- Continuity of care should be ensured for patients with craniofacial microsomia who reach adulthood.

\section{Centralisation}

- Patients with craniofacial microsomia are only treated for craniofacial microsomia-related difficulties in a centres that meets the criteria (including volume of care) defined by the ERN-CRANIO.

\section{Monitoring}

- Patient measure should be performed as stated in each chapter.

- Adhere to the ERN-CRANIO registry.

\section{CHAPTER 1. INTRODUCTION}

\subsection{Incentive for making the guideline}

Craniofacial microsomia (CFM) is estimated to occur in 1:3000 to 1:5000 live births and is the second most common congenital disorder of the face after cleft lip and palate (1). Diagnosis, treatment and outcome assessment is challenging due to a wide phenotypic spectrum (1). The diagnosis is based on clinical assessment, and no clear diagnosis criteria exist. As a result, treatment options vary within and among different European countries and are often based on expert opinion. So far, no international guideline has been developed. Since practice and expert opinions vary, it is relevant to discuss the available literature, current practice and current experiences with different healthcare professionals in Europe. An international guideline will result in a more aligned and uniform organisation of care for patients with CFM European countries.

\subsection{Purpose of the guideline}

There is a need to establish an international guideline regarding patients with CFM in collaboration with a number of European countries due to the wide phenotypic spectrum and variety of diagnostic criteria and treatment options for CFM. The guideline should fit the current practice in the countries involved and will give healthcare professionals tools to align and standardise healthcare in their own country and in other European countries.

The guideline can support healthcare professionals in discussing the use of certain techniques or instruments with other healthcare professionals or their national council. In addition, this guideline will provide CFM patients (and their parents) and healthcare professionals with an overview of the optimal care concerning the various and multidisciplinary aspects of craniofacial microsomia.

\subsection{Scope of the guideline}

The guideline focusses on all patients with CFM. This includes patients with Goldenhar syndrome, hemifacial microsomia, oculoauriculo-vertebral spectrum/dysplasia and facio-auriculo-vertebral sequence. The guideline will focus on the various and multidisciplinary aspects of CFM.

Recommendations on the following questions are provided in this guideline:

Chapter 3 - Diagnostic criteria for craniofacial microsomia

3.1 Based on which criteria is a child or adult with craniofacial microsomia diagnosed?

Chapter 4 - Screening, monitoring and indication for treatment

4.1 Breathing difficulties in craniofacial microsomia

4.2 Feeding difficulties in craniofacial microsomia

4.3 Speech difficulties in craniofacial microsomia

4.4 Hearing difficulties in craniofacial microsomia

4.5 Eye anomalies in craniofacial microsomia

4.6 Dental deformities in craniofacial microsomia

4.7 Vertebral anomalies in craniofacial microsomia

4.8 Psychosocial difficulties in craniofacial microsomia

Each chapter includes the following questions:

4.-.1 What is the type, prevalence and severity of .. difficulties in craniofacial microsomia?

4.-.2 What is the policy for screening and monitoring of .. difficulties in patients with craniofacial microsomia?

4.-.3 What are the indications and policy for treatment of .. difficulties in patients with craniofacial microsomia?

Chapter 5 - Surgical treatment of craniofacial microsomia 5.1 Mandible \& Maxilla

5.1.1 What is the indication for surgical treatment of mandibular and maxillary deformity in patients with craniofacial microsomia? 
5.1.2 What is the most optimal treatment modality and its timing for mandibular/maxillary deformity in craniofacial microsomia regarding severity, breathing difficulties, occlusal problems and aesthetics?

\subsection{Facial nerve}

5.2.1 What is the indication for surgical treatment of facial nerve anomaly in patients with craniofacial microsomia?

5.2.2 What is the most optimal treatment modality for facial nerve anomaly in patients with craniofacial microsomia related to functional deficits and aesthetics?

5.3 Soft tissues

5.3.1 What is the indication for surgical treatment of soft tissue deficiency in patients with craniofacial microsomia?

5.3.2 What is the most optimal treatment modality for soft tissue deficiency in patients with craniofacial microsomia related to severity and its timing?

\subsection{Microtia}

5.4.1 What is the indication for surgical treatment of ear deformity in patients with craniofacial microsomia?

5.4.2 What is the most optimal treatment modality for ear deformity in patients with craniofacial microsomia related to its timing?

Chapter 6 - Organisation of care

6.1 Minimal care standards and monitoring outcomes

6.1.1 What are the minimal care standards to treat patients with craniofacial microsomia and how should outcomes of care be monitored?

\subsection{Relationship to other congenital facial malformations}

The facial characteristics of patients with CFM show an overlap with other craniofacial anomalies, such as facial clefts or Treacher Collins (mandibulofacial dysostosis). These patients experience similar difficulties due to the underdevelopment of craniofacial structures, such as the mandible, midface, eyes and/or ears (2). This may include difficulties with breathing, feeding, speech, hearing, and/or developmental delay. Potential screening and treatment and the multidisciplinary approach needed for these patients has overlap with the policy for patients with CFM. This guideline might be helpful to organise and optimise care for patients with similar craniofacial characteristics.

\subsection{Intended users of the guideline}

This guideline is primarily written for all healthcare professionals involved in the care for patients with CFM, including: paediatricians, oral and maxillofacial surgeons, plastic surgeons, orthodontists, otorhinolaryngologists, neurosurgeons, orthopaedic surgeons, ophthalmologists, anaesthesiologists, geneticists, psychologists, and speech therapists. Secondly, this guideline is made to provide patients and parents or other persons who are involved in the medical care of adults or children with CFM with more information about the care process.

\subsection{About craniofacial microsomia}

Craniofacial microsomia (CFM) is one of the most common congenital conditions treated in craniofacial centres worldwide. It is a heterogeneous congenital disorder which is characterised by a unilateral or bilateral underdevelopment of the structures arising from the first and second pharyngeal arch. The mandible, zygoma, ears, facial soft tissue, orbits, and facial nerve may be underdeveloped in patients with CFM and extracranial anomalies such as vertebral, renal or cardiac anomalies may be present. The cause of this condition is unknown, though CFM has been associated with prenatal exposures and genetic abnormalities (1). No clear diagnostic criteria exist. Although microtia is common in patients with CFM, it is still debated in literature whether isolated microtia is a separate entity or part of the CFM 'spectrum' $(3,4)$.

\subsection{European Reference Networks}

European Reference Networks (ERNs) are virtual networks of healthcare providers from across Europe. The networks aim to pool expertise on complex and rare diseases and concentrate knowledge and resources. There are 24 ERNs, each focusing on a particular disease area. ERN-CRANIO focuses on rare and/or complex craniofacial anomalies and ear, nose and throat (ENT) disorders. More information and updates can be found on the website of the ERNCRANIO: https://ern-cranio.eu/

ERN-CRANIO seeks to facilitate cooperation between multidisciplinary experts across Europe to support the provision of highquality care. It is a multidisciplinary network of highly specialised healthcare professionals. The name 'subgroup' will be used when referring to all healthcare professionals incorporated in the ERNCRANIO.

\section{References}

1. Birgfeld CB, Heike C. Craniofacial microsomia. Semin Plast Surg. 2012;26(2):91-104.

2. Franceschetti A, Klein D. The mandibulofacial dysostosis; a new hereditary syndrome. Acta Ophthalmol (Copenh). 1949;27(2):143-224.

3. Bennun RD, Mulliken JB, Kaban LB, Murray JE. Microtia: a microform of hemifacial microsomia. Plast Reconstr Surg. 1985;76(6):859-65.

4. Barisic I, Odak L, Loane M, Garne E, Wellesley D, Calzolari E, et al. Prevalence, prenatal diagnosis and clinical features of oculo-auriculo-vertebral spectrum: a registry-based study in Europe. Eur J Hum Genet. 2014;22(8):1026-33.

\section{CHAPTER 2. METHODOLOGY}

\subsection{Validity of the guideline}

The board of the ERN-CRANIO will determine whether the guideline is still up to date in 2025 . If necessary, a new workgroup will be installed to review the guideline or some of its chapters. The validity of the guideline will expire sooner if new developments are of large influence on the current guideline.

The ERN-CRANIO is primarily responsible for the validity of the guideline. The cooperating associations share the responsibility and inform the ERN-CRANIO when new developments are of influence on the guideline.

\subsection{General information}

The development of this guideline was supported by Qualicura, an independent consultancy firm that develops medical guidelines.

\subsection{Aim and target audience guideline 2.3.1 Aim of the Guideline}

The aim of this guideline is to provide healthcare professionals and patients (and parents of patients) with craniofacial microsomia (CFM) with an overview of the optimal care concerning the various and multidisciplinary aspects of CFM and offer with recommendations to improve health outcomes and organisation of care. 


\subsubsection{Target audience}

- Healthcare professionals dealing with craniofacial microsomia

- Patients with craniofacial microsomia and parents of patients

\subsubsection{Patient population}

Patients with CFM or syndromes that are considered to be a variant of CFM. These are: hemifacial microsomia, Goldenhar syndrome, oculo-auriculo-vertebral spectrum/dysplasia or facioauriculo-vertebral sequence. According to the Orphanet, Snomed and ICD10 coding systems, this considers:

Orphanet: ORPHA:374; ORPHA:141132; ORPHA:141136; ORPHA: 2549

Snomed: 367462009; 109393007; 15557005; 205418005; 254026007; 254025006; 254027003

ICD10: Q75.8; Q87.0; Q75.9

\subsection{Steering group}

A multidisciplinary steering group was appointed to develop the guideline in November 2018. The members of the steering group are primarily members of the subgroup ERN-CRANIO. The guideline steering group consisted of eight professionals specialised in maxillofacial surgery and plastic surgery. Professionals represented the following countries: the Netherlands, the United Kingdom, France, Spain, and Finland. The guideline steering group was chaired by a maxillofacial surgeon. The literature search and the grading of the literature was performed by a research fellow, R.W. Renkema. The literature review and its conclusions were also written by R.W. Renkema. The review of literature and discussion with the steering group led to the final recommendations and considerations. These were written by R.W. Renkema. Qualicura was responsible for the coordination and methodological quality of the guideline development process. The steering group members were mandated by their professional organisation.

\section{Steering group}

- Prof. E.B. Wolvius, MD, DMD, PhD, maxillofacial surgeon, Erasmus Medical Center, Rotterdam, the Netherlands

- Prof. I.M.J. Mathijssen, MD, PhD, plastic surgeon, Erasmus Medical Center, Rotterdam, the Netherlands

- Dr. A.I. Romance, MD, DMD, maxillofacial surgeon, Hospital 12 de Octobre, Madrid, Spain
- Dr. M.S.M. Muradin, MD, DMD, PhD, Maxillofacial surgeon, Utrecht Medical Center, Utrecht, the Netherlands

- Dr. R.H. Khonsari, MD, PhD, maxillofacial surgeon, Hôpital Necker des Enfants Malades, Paris, France

- Dr. N.W. Bulstrode, MBBS, BSc(Hons), MD, FRCS(Plast), Plastic surgeon, Great Ormond Street Hospital, London, United Kingdom

- Dr. T. Pihlamaa, MD, PhD, plastic surgeon, Helsinki University Hospital, Helsinki, Finland

- Drs. R.W. Renkema, MD, research fellow, Erasmus Medical Center, Rotterdam, the Netherlands

Supported by

- Dr. L.F.J. Welling - van Overveld, MSc, PhD, guideline methodologist, Qualicura, Breda, the Netherlands

- Drs. V.R. Krones, MSc, guideline methodologist, Qualicura, Breda, the Netherlands

- Drs. E.L. Weissbach, nurse specialist, Erasmus Medical Center, Rotterdam, the Netherlands

Experts on each topic of the guideline were consulted to review the chapters and write recommendations:

- Dr. K. Joosten, MD, PhD, pediatrician, Erasmus Medical Center, Rotterdam, the Netherlands

- N. Prendeville, MRes, MSc, MRCSLT, Speech and Language therapist, Great Ormond Street Hospital NHS Foundation Trust, London, United Kingdom

- N. Behari, N. Behari, MECI, MRCSLT, speech and language therapist, Great Ormond Street Hospital NHS Foundation Trust, London, United Kingdom

- Dr. M.P. Van der Schroeff, MD, PhD, otolaryngologist, Erasmus Medical Center, Rotterdam, the Netherlands

- Dr. S.E. Loudon, MD, PhD, ophthalmologist, Erasmus Medical Center, Rotterdam, the Netherlands

- Dr. E. Ongkosuwito, DDS, PhD, orthodontist, Radboud University Medical Centre, Nijmegen, the Netherlands

- Dr. B.S. Harhangi, MD, PhD, neurosurgeon, Erasmus Medical Center, Rotterdam, the Netherlands

- Drs. C. Moffat, clinical psychologist, NHS Lothian, Scotland, United Kingdom

- Drs. N. Rooney, clinical psychologist, Great Ormond Street Hospital, London, United Kingdom

\subsection{Conflicts of interest}

All members of the steering group declared their conflicts of interest. An overview is given in Table 1.

\section{Table 1. Overview of conflicts of interest.}

\begin{tabular}{|c|c|c|c|c|c|c|c|}
\hline Name & Function & Ancillary activities & $\begin{array}{l}\text { Personal } \\
\text { financial } \\
\text { interests }\end{array}$ & $\begin{array}{l}\text { Personal } \\
\text { relations }\end{array}$ & $\begin{array}{l}\text { Externally } \\
\text { financed } \\
\text { research }\end{array}$ & $\begin{array}{l}\text { Intellectual } \\
\text { interest } \\
\text { and reputation }\end{array}$ & $\begin{array}{l}\text { Additional } \\
\text { interests }\end{array}$ \\
\hline Prof. E.B. Wolvius & Maxillofacial surgeon & $\begin{array}{l}\text { Part-time position at St. Anna } \\
\text { Hospital, Geldrop, The } \\
\text { Netherlands. } \\
\text { Chair of AOCMF Research \& } \\
\text { Development Commission, AO } \\
\text { Foundation }\end{array}$ & None & None & None & None & None \\
\hline Prof. I.M.J. Mathijssen & Plastic surgeon & Coordinator of ERN-CRANIO & None & None & None & None & None \\
\hline Dr. A.I. Romance & Maxillofacial surgeon & None & None & None & None & None & None \\
\hline
\end{tabular}




\begin{tabular}{|c|c|c|c|c|c|c|c|}
\hline \multicolumn{8}{|l|}{ (continued) } \\
\hline Name & Function & Ancillary activities & $\begin{array}{l}\text { Personal } \\
\text { financial } \\
\text { interests }\end{array}$ & $\begin{array}{l}\text { Personal } \\
\text { relations }\end{array}$ & $\begin{array}{l}\text { Externally } \\
\text { financed } \\
\text { research }\end{array}$ & $\begin{array}{l}\text { Intellectual } \\
\text { interest } \\
\text { and reputation }\end{array}$ & $\begin{array}{l}\text { Additional } \\
\text { interests }\end{array}$ \\
\hline Dr. M.S.M. Muradin & Maxillofacial surgeon & $\begin{array}{l}\text { President of Auditing Committee } \\
\text { of Dutch Society of Cleft Palate } \\
\text { and craniofacial surgery } \\
\text { (NVSCA) } \\
\text { Treasurer of NVSCA } \\
\text { (nov 2019) } \\
\text { Treasurer ECPCA } \\
\text { Instructor Human Cadaver } \\
\text { Course, Implant } \\
\text { college }\end{array}$ & None & None & None & None & None \\
\hline Dr. R.H. Khonsari & Maxillofacial surgeon & None & None & None & None & None & None \\
\hline Dr. N.W. Bulstrode & Plastic surgeon & Will follow & Will follow & Will follow & Will follow & Will follow & Will follow \\
\hline Dr. T. Pihlamaa & Plastic surgeon & $\begin{array}{l}\text { Private practice, Plastic } \\
\text { surgeon, Pihlajalinna } \\
\text { Tilkka Hospital \& Plastic } \\
\text { Surgery Center, Helsinki, } \\
\text { Finland }\end{array}$ & None & None & None & None & None \\
\hline Drs. R.W. Renkema & Research fellow & None & None & None & None & None & None \\
\hline $\begin{array}{l}\text { Dr. L.F.J. Welling - van } \\
\text { Overveld }\end{array}$ & $\begin{array}{l}\text { Guideline } \\
\text { methodologist }\end{array}$ & None & None & None & None & None & None \\
\hline Drs. V.R. Krones & Guideline methodologist & None & None & None & None & None & None \\
\hline Prof. dr. K. Joosten & Paediatric-intensivist & None & None & None & None & None & None \\
\hline N. Prendeville & $\begin{array}{c}\text { Specialist speech and } \\
\text { language therapist }\end{array}$ & None & None & None & None & None & None \\
\hline N. Behari & $\begin{array}{c}\text { Specialist speech and } \\
\text { language therapist }\end{array}$ & None & None & None & None & None & None \\
\hline $\begin{array}{l}\text { Dr. M.P. Van der } \\
\text { Schroeff }\end{array}$ & Otolaryngologist & None & None & None & None & None & None \\
\hline Dr. S.E. Loudon & $\begin{array}{l}\text { Paediatric } \\
\text { ophthalmologist }\end{array}$ & None & None & None & None & None & None \\
\hline Dr. E. Ongkosuwito & Orthodontist & $\begin{array}{l}\text { Deputy director of the } \\
\text { Postgraduate training in } \\
\text { Orthodontics and section of } \\
\text { Orthodontics and Craniofacial } \\
\text { Biology, Department of } \\
\text { Dentistry, Radboud University } \\
\text { Medical Center, Nijmegen, the } \\
\text { Netherlands } \\
\text { Private practice in Orthodontics, } \\
\text { Orthopraktijk Capelle, } \\
\text { Capelle aan den ljssel, The } \\
\text { Netherlands }\end{array}$ & None & None & None & None & None \\
\hline Dr. B.S. Harhangi & Neurosurgeon & None & None & None & None & None & None \\
\hline Dr. C. Moffat & Clinical psychologist & Will follow & Will follow & Will follow & Will follow & Will follow & Will follow \\
\hline Dr. N. Rooney & Clinical psychologist & None & None & None & None & None & None \\
\hline
\end{tabular}




\subsection{Patient perspectives}

Since the guideline will be developed for patients and parents of patients, the patient perspective will be of major importance in this guideline. The perspective of patients was included by analysing relevant bottlenecks from the online survey (see Chapter 2.8.1).

\subsection{Implementation}

The implementation of the guideline and the practical feasibility of the recommendations were taken into account during the different phases of guideline development. In doing so, explicit consideration was given to factors that could promote or hinder the implementation of the guideline in practice.

\subsection{Methods}

\subsubsection{Bottleneck analysis}

A draft list of bottlenecks from a professional perspective was written by the chair and vice chair. Members of the steering group were asked to give feedback on the draft bottleneck analysis. The first set of bottlenecks were discussed during the first international conference in February 2019. No additional chapters were added regarding the bottlenecks of the professionals.

All doctors included in the ERN-CRANIO, subgroup 'craniofacial microsomia', were asked to approach their CFM patients. This led to the identification of a group of 32 interviewees: 14 from Italy, 13 from Germany, 4 from the Netherlands, and 1 from Sweden, including 9 patients and 23 parents of patients. An online survey was set up with open and closed questions. All patients and parents of patients were asked what difficulties they (had) experienced in the healthcare process and in their lives. The questionnaire was built up according to the proposed guideline chapters and the healthcare process, namely diagnosis and referral, organisation of care, communication and information, breathing difficulties, feeding difficulties or speech difficulties, surgical treatments, care for microtia, orthodontic treatment, vertebral anomalies, psychosocial aspects of care, and follow-up. Additionally, all patients were asked to name the top three difficulties they experienced in the care process. Results were analysed by the research fellow (R.W. Renkema) and nurse specialist (E.L. Weissbach). Most frequently mentioned difficulties included difficulties in receiving adequate information on the diagnosis and the treatment, difficulties in getting referred to an experienced medical centre, and absence of psychological care. Based on the patient perspectives, a chapter on psychosocial difficulties was added. Other relevant bottlenecks were included in the chapter on organisation of care.

Bottlenecks of both patients and specialists were forwarded to relevant people in participating countries in March 2019, giving them the opportunity to give feedback on the bottlenecks. Afterwards, a draft framework of the guideline was set up and questions and outcomes were formulated.

\subsubsection{Questions and outcomes}

The bottleneck analysis formed the basis for the questions for the guideline. The questions were formed according to the PICOT Framework and presented in each guideline chapter. To maintain a clear and readable chapter, questions in the guideline were formulated in a broad and clinically relevant way. The terms for and more specified questions to facilitate the literature search were specified in the summary of literature and Appendix 1, http://links.lww.com/ SCS/B697.

The guideline is divided into a non-surgical and surgical part. Questions for the non-surgical chapters (Chapter 4 (4.1 to 4.8)) are formulated in a similar way. Likewise, questions for the surgical chapters (Chapter 5 (5.1 to 5.4)) are also formulated in a similar way.

For questions in the surgical chapters specific patient outcomes such as aesthetic results, quality of life, or complications were formulated. The patient outcomes were described in the summary of literature. Patient outcomes were taken into account when formulating the recommendations. In addition, patients' outcomes were included in the summary of literature when they were reported in literature.

\subsubsection{Literature search and selection of literature}

One systematic search of literature was performed to identify all available literature on craniofacial microsomia and synonyms. The search was conducted in Embase, Pubmed/Medline Ovid. The full search strategy is reported in the supplementary material.

Inclusion and exclusion criteria:

Type of studies

- Original articles

- Systematic review of sufficient quality:

- The question in the systematic review matches the question of the guideline.

- The search of the systematic review was conducted in at least two relevant databases, such as the Cochrane Library, Medline/Pubmed.

- The full search strategy was reported.

- No relevant items were missing in the search strategy.

Type of patients

- Patients with craniofacial microsomia

Subject

- Psychosocial functioning/ difficulties, neurodevelopment

Exclusion criteria

- Original studies with $<10$ included patients

- Articles published before 1980

- Case reports

- Expert opinion

- Letters

- Editorials

- Narrative reviews

A total of 1,747 articles were screened on title and abstract. Most articles $(1,488)$ were excluded and 259 articles were reviewed on full text. A total of 101 articles were included in the guideline.

The selected studies were categorised according to the framework of the guideline.

No narrative reviews were taken into account except for Chapter $4.5,5.2$, and 5.3. Since there was hardly any evidence, the available narrative review was of importance.

For a couple of chapters, hardly no evidence was found. In these cases an additional literature search was performed, regarding a specific treatment in a different patient population for example. The full extra search strategy is reported in the supplementary material. Table 5 gives an overview of the chapters for which an additional literature search was performed. In a couple of chapters, additional literature is only included in the considerations and/or the rationale. 


\subsubsection{Quality assessment of individual studies}

Individual studies were systematically assessed, based on preestablished methodological quality criteria, in order to estimate the risk of biased study results. The evidence table of all individual studies is displayed in Appendix 6, http://links.lww.com/SCS/ B697.

\subsubsection{Summary of literature}

The most important findings from the literature were described in the summary of literature. Literature with a high risk of bias was found for a number of chapters and hardly any evidence was found for a couple of chapters. Percentages were rounded in the conclusions. The steering group decided to include expert opinions for the chapters with hardly any evidence. Therefore, specific experts on each topic of the guideline were consulted to review the chapter. In addition, experts were asked to write considerations and recommendations to initiate the discussion during the meeting in September 2019. In the end, all written text was discussed during the meeting in September 2019.

\subsubsection{Quality of evidence}

The quality of evidence for included studies was assessed using the EBRO method. The methodological quality of individual studies was categorised in five levels (Table 2), the level of evidence was categorised in four levels (Table 3 ) and grading of the study was categorised in four levels (Table 4). The level of conclusion was not assessed for studies referring to prevalence.

Table 2. Classification of methodological quality

\begin{tabular}{|c|c|c|c|}
\hline & Intervention & $\begin{array}{l}\text { Diagnostic accuracy } \\
\text { research }\end{array}$ & $\begin{array}{l}\text { Side effects", aetiology, } \\
\text { prognosis }\end{array}$ \\
\hline A1 & \multicolumn{3}{|c|}{ Systematic review of at least two independent studies of the level A2 } \\
\hline A2 & $\begin{array}{l}\text { Randomised, double } \\
\text { blind, comparative } \\
\text { clinical research of } \\
\text { good quality and } \\
\text { with adequate size. }\end{array}$ & $\begin{array}{l}\text { Research compared } \\
\text { with a reference } \\
\text { test (golden } \\
\text { standard) with } \\
\text { predefined cut-off } \\
\text { values and } \\
\text { independent rating } \\
\text { of results and the } \\
\text { golden standard, } \\
\text { with an adequate } \\
\text { number of patients } \\
\text { who have all had } \\
\text { the index and the } \\
\text { reference test. }\end{array}$ & $\begin{array}{l}\text { Prospective cohort } \\
\text { research of adequate } \\
\text { size and follow-up } \\
\text { with adequate control } \\
\text { for confounding and } \\
\text { selective follow-up is } \\
\text { sufficiently excluded. }\end{array}$ \\
\hline $\mathrm{B}$ & $\begin{array}{l}\text { Comparative } \\
\text { research but not } \\
\text { with all the } \\
\text { characteristics } \\
\text { included in A2 } \\
\text { (including patient- } \\
\text { control research } \\
\text { and cohort } \\
\text { research). }\end{array}$ & $\begin{array}{l}\text { Research compared } \\
\text { with a reference } \\
\text { test but not with all } \\
\text { characteristics } \\
\text { included in A2. }\end{array}$ & $\begin{array}{l}\text { Prospective cohort } \\
\text { research but not with } \\
\text { all characteristics } \\
\text { included in A2 or a } \\
\text { retrospective cohort } \\
\text { research or patient- } \\
\text { control research. }\end{array}$ \\
\hline $\mathrm{C}$ & \multicolumn{3}{|c|}{ Not comparative research } \\
\hline $\mathrm{D}$ & \multicolumn{3}{|c|}{ Opinion of experts } \\
\hline
\end{tabular}

*This classification only applies in situations were controlled trials are not possible for ethical reasons. If they are possible, then the classification applies to interventions
Table 3. Level of conclusion

\begin{tabular}{ll}
\hline & Conclusion based on \\
\hline 1 & $\begin{array}{c}\text { Research of level A1 or at least two independent } \\
\text { studies of level A2 }\end{array}$ \\
\hline 3 & $\begin{array}{c}\text { One study of level A2 or at least two independent } \\
\text { studies of level B }\end{array}$ \\
\hline 4 & One study of level B or C \\
\hline
\end{tabular}

Table 4. Grading of qualitative research

\begin{tabular}{ll}
\hline Level & Study \\
\hline+ & $\begin{array}{c}\text { Credible meta-synthesis (meta-ethnography, qualitative meta-analysis, } \\
\text { meta-study) of qualitative studies }\end{array}$ \\
\hline+ & Credible studies \\
\hline$+/-$ & Studies in which the credibility is questionable \\
\hline & Studies of low credibility \\
\hline
\end{tabular}

\subsubsection{Formulating conclusions}

For each chapter a conclusion was given when literature was available. Conclusions are drawn on the basis of all studies combined. If no literature was available, no conclusions were drawn.

\subsubsection{Considerations}

To determine the strength and direction of a recommendation, the following aspects were examined in addition to the quality of evidence:

- Balance of benefits and harms

- Outcome importance

- Costs and resources

- Inequity of the recommendation

- Feasibility of the recommendation

- Acceptability of the recommendation

Conclusions were mostly written by the fellow (R.W. Renkema) based on the available literature. When no evidence was available, experts were consulted to write draft considerations. The draft considerations were discussed in the meeting in September 2019.

The steering group decided to include expert opinions for the chapters with hardly any evidence. Therefore, specific experts on each topic of the guideline were consulted to review the chapters and to write recommendations and considerations to initiate the discussion in September 2019. In the end, all written text was reviewed and discussed by the steering group. Table 5 gives an overview of the chapters with recommendations written by an expert. For the remaining chapters, the draft recommendations were written by the fellow (R.W. Renkema). 
Table 5. Overview of the source of information for each chapter.

Chapter

3. Diagnostic criteria

3.1 Based on which criteria is a child or adult with craniofacial microsomia diagnosed?

4.1 Breathing difficulties

4.1.1 What is the type, prevalence and severity of breathing difficulties in craniofacial microsomia?

4.1.2 What is the policy for screening and monitoring of breathing difficulties (OSA) in patients with craniofacial microsomia?

4.1.3 What are the indications and policy for treatment of breathing difficulties (OSA) in patients with craniofacial microsomia?

4.2 Feeding difficulties

4.2.1 What is the type, prevalence and severity of feeding difficulties in craniofacial microsomia?

4.2.2 What is the policy for screening and monitoring of feeding difficulties (OSA) in patients with craniofacial microsomia?

4.2.3 What are the indications and policy for treatment of feeding difficulties (OSA) in patients with craniofacial microsomia?

4.3 Speech difficulties

4.3.1 What is the type, prevalence and severity of speech difficulties in craniofacial microsomia?

4.3.2 What is the policy for screening and monitoring of speech difficulties in patients with craniofacial microsomia?

4.3.3 What are the indications and policy for treatment of speech difficulties in patients with craniofacial microsomia?

4.4 Hearing difficulties

4.4.1 What is the type, prevalence and severity of hearing difficulties in craniofacial microsomia?

4.4.2 What is the policy for screening and monitoring of hearing difficulties in patients with craniofacial microsomia?

4.4.3 What are the indications and policy for treatment of hearing difficulties in patients with craniofacial microsomia?

4.5 Eye anomalies

4.5.1 What is the type, prevalence and severity of eye anomalies in craniofacial microsomia?

4.5.2 What is the policy for screening and monitoring of eye anomalies in patients with craniofacial microsomia?

4.5.3 What are the indications and policy for treatment of eye anomalies in patients with craniofacia microsomia?

4.6 Dental deformities

4.6.1 What is the type, prevalence and severity of dental deformities in craniofacial microsomia?

4.6.2 What is the policy for screening and monitoring of dental deformities in patients with craniofacial microsomia?

4.6.3 What are the indications and policy for treatment of dental deformities in patients with craniofacial microsomia?

4.7 Vertebral anomalies

4.7.1 What is the type, prevalence and severity of vertebral deformities in craniofacial microsomia?

4.7.2 What is the policy for screening and monitoring of vertebral deformities in patients with craniofacial microsomia?

4.7.3 What are the indications and policy for treatment of vertebral deformities in patients with craniofacial microsomia?

4.8 Psychosocial difficulties

4.8.1 What is the type, prevalence and severity of psychosocial difficulties in craniofacial microsomia?

4.8.2 What is the policy for screening and monitoring of psychosocial difficulties in patients with craniofacial microsomia?

4.8.3 What are the indications and policy for treatment of psychosocial difficulties in patients with craniofacial microsomia?

5.1 Mandible and Maxilla

5.1.1 What is the indication for surgical treatment of mandibular and maxillary deformity in patients with craniofacial microsomia?

5.1.2 What is the most optimal treatment modality and its timing for mandibular/maxillary deformity in craniofacial microsomia regarding severity, breathing problems, occlusal problems and aesthetics?

5.2 Facial nerve

5.2.1 What is the indication for surgical treatment of facial nerve anomaly in patients with craniofacial microsomia?

5.2.2 What is the most optimal treatment modality for facial nerve anomaly in patients with craniofacial microsomia related to functional deficits and aesthetics?

5.3 Soft tissue

5.3.1 What is the indication for surgical treatment of soft tissue deficiency in patients with craniofacial microsomia?

5.3.2 What is the most optimal treatment modality for soft tissue deficiency in patients with craniofacial microsomia related to severity and its timing?

5.4 Microtia

5.4.1 What is the indication for surgical treatment of ear deformity in patients with CFM?

5.4.2 What is the most optimal treatment modality for ear deformity in patients with CFM related to its timing?

$\begin{aligned} & \text { Literature } \\ & \text { available? } \\ & \text { Yes }\end{aligned}$
Yes, except for type of
breathing difficulties
No
Yes, except for indications of
breathing difficulties. That
question is answered by
including literature about
treatment modalities.

Draft considerations and recommendations written by experts

Additional search performed

Dr. K. Joosten

Dr. K. Joosten$$
\text { Yes }
$$

No

Yes

Yes

No

No

Yes

Yes

No

Yes

No

Additional literature

was included

Yes

No

Yes

Yes

No
Yes

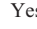

No

Yes

Yes

Dutch guideline was used Yes

No

Yes
N. Prendeville, MRes, MSc, MRCSLT

N. Behari, MECI, MRCSLT

Dr. M.P. van der Schroeff

Dr. S.E. Loudon

epibulbar

dermoids and variants
Dr. E. Ongkosuwito

Dr. B.S. Harhangi

Drs. C. Moffat

Drs. N. Rooney
Additional literature search on the treatment for retro/ micrognathia was performed

Dutch guideline on peripheral facial palsy was additionally included combined with an extra literature search 


\subsubsection{Formulating recommendations}

The recommendations provide an answer to the basic question and are based on the best available scientific evidence and the most important considerations. The strength of the scientific evidence and the weight that the working group assigns to the considerations together determine the strength of the recommendation. In accordance with the EBRO method, a low probative value of conclusions in the systematic literature analysis does not exclude a strong recommendation in advance, and weak recommendations are also possible with a high probative value. The strength of the recommendation is always determined by weighing all relevant arguments.

Recommendations were mostly written by the fellow (R.W. Renkema) based on the available literature. When no evidence was available, experts were consulted to write draft recommendations. The draft recommendations were discussed in the meeting in September 2019.

\subsubsection{Conditions (organisation of care)}

The bottleneck analysis and the development of the guideline explicitly take into account the aspects related to organisation of care. This contains all aspects that are preconditions for providing care, such as coordination, communication, (financial) resources, manpower and infrastructure. More general, overarching, and additional aspects of the organisation of care are discussed in Chapter 6.

\subsubsection{Knowledge gap}

During the development of this guideline a systematic literature search was performed to answer the questions. For each question the steering group investigated whether (additional) scientific research is necessary. An overview of recommendations for further research can be found in Chapter 7 .

\subsubsection{Evaluation and authorisation phase}

The draft guideline was submitted to all craniofacial centres involved in the care for patients with CFM and affiliated with the ERN-CRANIO. The comments were collected and discussed with the steering group. As a result of the comments, the draft guideline was adapted and finally adopted by the steering group.

\section{CHAPTER 3. DIAGNOSTIC CRITERIA FOR CRANIOFACIAL MICROSOMIA}

\section{Introduction}

In patients with craniofacial microsomia (CFM), the facial structures arising from the first and second pharyngeal arches may be underdeveloped. The characteristic features are malformation, hypoplasia or aplasia of the outer and inner ear, orbit, zygoma, maxilla, mandible, facial musculature, facial nerve, and/or soft tissue, but extracraniofacial anomalies may be present as well $(1,2)$. The variety in type and severity of underdevelopment of these structures make CFM a heterogeneous disorder. The diagnosis of CFM is based on clinical assessment, though no clear diagnostic criteria exist.

Various classification models have been proposed to categorise patients with CFM based on its severity (3-8). The most commonly used system is the O.M.E.N.S. classification, which describes the degree of hypoplasia of the Orbit (O), Mandible (M), Ears (E), Facial Nerve $(\mathrm{N})$, and Soft Tissue $(\mathrm{S})(2,3)$. Extracraniofacial anomalies, facial clefting, canting of the occlusal plane, and detailed assessment of eye and ear anomalies is included in this classification as well $(2,9)$. A more detailed classification of the mandibular deformity, based on radiography, was proposed by Pruzansky and later subcategorised by $\operatorname{Kaban}(5,10,11)$. In this classification model the level of underdevelopment of the mandible is graded as I, IIA, IIB, and III.

A wide range of terminology is used to refer to patients with CFM: hemifacial microsomia, Goldenhar syndrome, oculo-auriculo-vertebral spectrum or dysplasia, first and second branchial arch syndrome, or facio-auriculo-vertebral syndrome or sequence.

The term CFM is preferred for two main reasons. Firstly, as slightly more than $10 \%$ of the patients with CFM are bilaterally affected the term hemifacial microsomia (HFM), which has been commonly used, is not considered to cover the extensiveness of the syndrome $(9,12)$. Secondly, the side that has the more significant hypoplasia causes deformity on the contralateral side making this a condition that affects both sides. Therefore CFM is a more precise term.

Microtia is an important and common aspect of patients with CFM. Whether patients with isolated microtia fit the diagnosis CFM is still being debated $(1,4,13,14)$. Some authors advocate the inclusion of patients with isolated microtia as CFM, as the cause and types of ear malformations are similar in both groups. In addition, the incidence of ear malformations found in family members of patients with CFM is much higher than in the general population (15). However, no consensus has been reached.

Goldenhar syndrome was originally regarded as an association of mandibular dysostosis, ear malformations and epibulbar dermoids (16). Although there are no clear diagnostic criteria for Goldenhar syndrome, currently the diagnosis is often seen as CFM with the additional presence of epibulbar dermoids and vertebral/cervical spine anomalies (the triad of Goldenhar). Some consider the diagnosis Goldenhar syndrome to be an entity distinct from CFM, while others include Goldenhar syndrome as a variant of CFM $(15,17)$. However, no consensus exists.

Another term used to describe patients with CFM is the oculoauriculo-vertebral spectrum or dysplasia (OAVS) (18). OAVS is often used to describe the expanded spectrum of CFM including facial malformations such as macrostomia or epibulbar dermoids, and extracraniofacial anomalies. Again, no consensus on diagnostic criteria exists.

The use of different terminology and lack of diagnostic criteria is confusing for patients and healthcare professionals. Therefore, the following question was posed:

\subsection{On which criteria is a child or adult with craniofacial microsomia diagnosed?}

Uniform terminology would be helpful to provide better information to patients and increase awareness of the disorder. Additionally, it would be beneficial for the communication between professionals and patients, between healthcare professionals, and in research projects.

\section{Literature search}

A systematic search was performed to identify all available literature on craniofacial microsomia and synonyms. The search was conducted in Embase, Pubmed/Medline Ovid. The full search strategy is reported in the supplementary material.

\footnotetext{
Inclusion and exclusion criteria:

Type of studies - Original articles

- Systematic review of sufficient quality:

- The question in the systematic review matches the question of the guideline.

- The search of the systematic review was conducted in at least two relevant databases, such as the Cochrane Library, Medline/Pubmed.

- The full search strategy was reported.

- No relevant items were missing in the search strategy.

Type of patients - Patients with craniofacial microsomia
} 


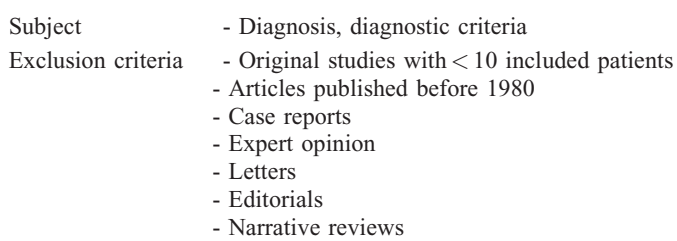

All articles were screened on title and abstract. Literature on the diagnosis of CFM or synonyms was included. A total of four articles were included: a case-control study by Heike et al. (19), a retrospective cohort study by Caron et al. (20), and two retrospective cohort studies by Tuin et al. $(21,22)$. Additional documents on diagnostic criteria available in the International Consortium for Health Outcomes Measurement (ICHOM) Craniofacial Microsomia were included as well (23).

\subsection{Based on which criteria is a child or adult with craniofacial microsomia diagnosed?}

The literature search for this question was directed towards more specific questions related to classification scores, the correlation of abnormalities, clusters within CFM, and Goldenhar syndrome (Appendix 1, http://links.lww.com/SCS/B697).

\section{Review of literature Classification score and clusters within craniofacial microsomia}

A case-control study, set up by Heike et al. (19), aimed to develop a standardised approach to assess and describe facial characteristics of a study cohort. A total of 142 patients with CFM and 316 healthy controls were included. The assessment of the O.M.E.N.S. score was performed in both groups. This led to the observation that orbital displacement $(\mathrm{O})$ was found in $40 \%$ of the patients with CFM, but in $20 \%$ of the controls as well. Abnormal orbital size was less frequently found in controls (1\%), but in $13 \%$ of the patients with CFM. The authors advocate a threshold for an 'affected' orbit $(\mathrm{O})$ that requires both displacement and an abnormal size, consistent with an ' $\mathrm{O}-3$ ' score. Mild mandibular hypoplasia (M) was present in $25 \%$ of the cases and $12 \%$ of the controls. Therefore, the threshold for mandibular hypoplasia (M) was set on grade 2 or higher. Similarly, mild ear malformations (E) were present in $15 \%$ of the cases and $5 \%$ of the controls and the threshold for microtia (E) was set on grade 2 or higher. The threshold for soft tissue deformity (S), which was present in $34 \%$ of the patients and $5 \%$ of the controls, was set on grade 2 or higher. These results show that mild facial deformities are also present in the normal population, which should be taken into account in diagnosing CFM. The authors define four groups of CFM (19). First, the patients with microtia only. Secondly the patients with microtia and mandibular hypoplasia. Thirdly, patients with more than two CFM features (i.e. epibulbar dermoid, facial tags, macrostomia, orbital deformity, facial nerve palsy), but not with the combination of both microtia and mandibular hypoplasia. And lastly, an atypical CFM group that has fewer than two CFM features and no microtia.

The components of the O.M.E.N.S score in patients with CFM were studied by Tuin et al. (21). Their retrospective study included 105 patients. Most of the included patients had an ear deformity (92\%), mandibular abnormality (88\%) and/or soft tissue deficiency (77\%). Facial nerve abnormalities and orbital deficiency or displacement were less common, and present in $39 \%$ and $28 \%$ of the patients, respectively. Logistic regression showed a significant correlation between the orbit, mandible and soft tissue score; between the soft tissue score and the ear deformity; and between the ear deformity and facial nerve. This study shows that there may be clusters of patients within CFM. The authors suggest that this may be due to their shared origin, since the orbit, mandible and soft tissue originate from the first pharyngeal arch, and the ear and facial nerve from the second pharyngeal arch (21).

To study any clusters within CFM, a large retrospective study was performed by Caron et al. (20). A total of 755 patients were included. In this study, mandible ( $91 \%$ of the patients), soft tissue ( $82 \%$ ) and ear $(83 \%)$ deformities were most common. Orbital malformation was present in $45 \%$ of the patients. The facial nerve score could only be assessed in 238 patients (32\%), of which $34 \%$ had a paresis. The authors investigated the presence of any clusters within the studied cohort by using principle component analysis. A correlation between the severity of the mandibular deformity, orbital deformity and soft tissue deficiency was found. The analysis did not show any specific clusters within CFM. Interestingly, an analysis on patients with data on the 'triad of Goldenhar' (mandibular hypoplasia, epibulbar dermoids and vertebral anomalies) showed no correlation among these three variables. Therefore, the authors conclude that the term Goldenhar syndrome should be discarded. Also, the absence of clusters suggest that CFM is a continuum of anomalies including different types and severities (20).

\section{Goldenhar syndrome}

The use of the term Goldenhar syndrome in general practice was assessed in more detail by Tuin et al. (22). A total of 138 patients with CFM were retrospectively included. Thirty-four percent of the patients had vertebral anomalies, $17 \%$ epibulbar dermoids and $7.2 \%$ a combination of these. Although only $7.2 \%$ of the patients fit the 'criteria' for Goldenhar syndrome, $32 \%$ were diagnosed with the syndrome according to clinical records. Eighty percent of the patients with epibulbar dermoids and vertebral anomalies were diagnosed with Goldenhar syndrome. The authors found that the term Goldenhar syndrome was used significantly more often for patients with bilateral CFM, or a more severely affected mandible or soft tissue deformity. Patients with epibulbar dermoids and vertebral anomalies had no differences in severity of the facial deformities compared to the patients without these anomalies. The authors conclude that the term Goldenhar syndrome is often misused for patients with a more severe form of CFM and show that Goldenhar syndrome is part of the CFM spectrum instead of a distinct condition (22).

\section{International criteria}

The International Consortium for Health Outcomes Measurement (ICHOM) started an initiative to gather prospective information on patients with CFM on set time points (23). The members of the ICHOM Craniofacial Microsomia working group consisted of healthcare professionals from around the world active in the care of patients with CFM. This group reached consensus on diagnostic criteria for CFM, to be used to include patients in their dataset. The following diagnostic criteria are used:

Table 1. Diagnostic criteria CFM, by ICHOM Craniofacial Microsomia (23)

\begin{tabular}{ll}
\hline CFM defined as: & 2 major criteria, or \\
& 1 major +1 minor criteria, or \\
& $3+$ minor criteria \\
Major criteria & Mandibular hypoplasia \\
& Microtia \\
& Orbital / facial bone hypoplasia \\
& Asymmetric facial movement \\
Minor criteria & Facial soft tissue deficiency \\
& Pre-auricular tags \\
& Macrostomia \\
& Clefting \\
& Epibulbar dermoids \\
& Hemivertebrae \\
\hline
\end{tabular}




\section{Conclusions}

Level 3 Correlation and clusters
$\begin{aligned} & \text { In patients with craniofacial microsomia the mandibular } \\ & \text { deformity, orbital deformity and soft tissue deficiency are } \\ & \text { correlated. However, no specific clusters of patient groups } \\ & \text { within craniofacial microsomia are present, indicating } \\ & \text { craniofacial microsomia is a continum of anomalies. } \\ & \operatorname{Ref}(20,21)\end{aligned}$

Level 3

Goldenhar syndrome
Goldenhar syndrome is not a separate diagnosis from craniofacial
microsomia but is part of the phenotypic spectrum of
craniofacial microsomia:
• Clinically, patients diagnosed with Goldenhar syndrome
have a more severe facial deformity compared to patients with
craniofacial microsomia.
• Objectively, by using the O.M.E.N.S. score, no
differences are present in the severity of facial anomalies in
patients with craniofacial microsomia compared to patients
with the triad of Goldenhar syndrome (craniofacial
microsomia + epibulbar dermoids + vertebral anomalies).
• No correlation exist between the triad of Goldenhar
syndrome (craniofacial microsomia + epibulbar dermoids +
vertebral anomalies).
• A substantial number of patients with the triad of
Goldenhar syndrome (craniofacial microsomia + epibulbar
dermoids + vertebral anomalies) are not diagnosed with
Goldenhar syndrome.
Ref (20, 22)

\section{Considerations}

\section{- Overall quality of evidence}

Two studies reported on correlations of facial deformities in patients with CFM, based on the O.M.E.N.S. classification. The correlation between the facial deformities was reported in both studies. The studies are descriptive retrospective cohort studies and were therefore graded on level $3(20,21)$.

Concerning the conclusions on Goldenhar syndrome, two studies were included as well. Both studies showed the absence of a correlation between the triad of Goldenhar syndrome and concluded that Goldenhar syndrome is part of CFM, instead of a variant. The studies are descriptive retrospective cohort studies and were therefore graded on level $3(20,22)$.

The diagnostic criteria proposed by the ICHOM Craniofacial Microsomia group were based on consensus between healthcare professionals (23). No research has been done to test the criteria. Since CFM is a clinical diagnosis and no specific criteria exist for CFM, verifying the criteria may be difficult. Since the criteria are based on consensus and expert opinion, the evidence is graded on level 5.

\section{- Balance of benefits and harms}

No harm is produced for the patients with CFM by applying the diagnostic criteria. Unambiguous use of diagnostic criteria and terminology promotes clarity for both patients and healthcare professionals. Standardised use of the term CFM, by using clear diagnostic criteria, helps patients receive the right diagnosis and treatment, and improves access to information about the disease. In research, clear use of terminology promotes the exchange of ideas and findings.

\section{- Outcome importance}

The use of diagnostic criteria for CFM is considered to be of importance to healthcare professionals to improve communication, research, and provision of information to patients. Several recent studies confirm that CFM is a continuum of anomalies and discourage the use of the term Goldenhar syndrome. The consensus of this steering group is to solely use the term CFM.

\section{- Costs and resources}

The impact on costs and resources of the given recommendations will vary per member state, depending on the available care providers and facilities. The recommendations concern the essential requirements for adequate treatment of patients with CFM and should thus be implemented. Costs are lowest and resources are most efficiently used when care for craniofacial disorders is centralised in a limited number of expert centres per member state. A general rule that can be applied is one expert centre per 10 million inhabitants.

\section{- Inequity of the recommendation}

The goal of the European Reference Networks (ERNs) is to eliminate inequality within Europe with regard to care for patients with rare diseases. At present, not every member state offers an expert centre for CFM, or the level of provided care does not (yet) meet all the requirements that are outlined in this guideline. By defining the baseline of required care for CFM, this guideline will help these member states to reach the appropriate level. The ERN on craniofacial anomalies and ENT disorders (ERN-CRANIO) can guide a patient in Europe to the available centres of expertise (www.ern-cranio.eu) and can support care providers with diagnosis and treatment advice.

\section{- Feasibility of the recommendation}

Recommendations refer to the general requirements for delivering optimal healthcare and are discussed with members from participating European countries. Quality of care was paramount in the discussions. Centralisation is proposed as one of the core values. However, in some countries the national organisation of healthcare might impede centralisation. National implementation of the ERNs that fits the situation of each country is necessary. For the member states with the lowest number of inhabitants, the establishment of a craniofacial centre might not be feasible, and collaboration with a craniofacial centre in the surrounding countries can be an option.

\section{- Acceptability of the recommendation}

It is expected that all stakeholders want to apply and will be applying the recommendations, because they are employed in ERNacknowledged institutions. National implementation plans are necessary to ensure that recommendations fit the situation in each country. In addition, not all countries participating in the ERNCRANIO are represented in the guideline development group, and new members will join within the coming years. For these countries, acceptance and implementation of ERN guidelines such as this guideline on CFM is obligatory.

\section{Rationale of the recommendations}

Since the evidence suggests no difference between CFM and Goldenhar syndrome, OAVS, or hemifacial microsomia, the steering group advocate the use of a single term: CFM. To increase awareness of this diagnosis and provide clarity for patients and healthcare professionals, the diagnostic criteria agreed on by the 'ICHOM Craniofacial Microsomia' working group are recommended for use in practice. 


\section{Recommendations}

Terminology

- It is advised to exclusively use the term craniofacial microsomia. Discard the use of other terms such as Goldenhar syndrome, hemifacial microsomia or auriculo-oculo-vertebral spectrum.

\begin{tabular}{ll}
$\begin{array}{l}\text { Diagnostic criteria } \\
\text { It is advised to use the diagnostic criteria for craniofacial microsomia developed } \\
\text { by the ICHOM Craniofacial Microsomia group. }\end{array}$ \\
\hline CFM is defined by: & $\begin{array}{l}2 \text { major criteria, or } \\
1 \text { major }+1 \text { minor criteria, or } \\
3+\text { minor criteria }\end{array}$ \\
\hline Major criteria & $\begin{array}{l}\text { Mandibular hypoplasia } \\
\text { Microtia } \\
\text { Orbital / facial bone hypoplasia } \\
\text { Asymmetric facial movement }\end{array}$ \\
\hline Minor criteria & Facial soft tissue deficiency \\
Pre-auricular tags \\
Macrostomia \\
Clefting \\
Epibulbar dermoids \\
Hemivertebrae
\end{tabular}

\section{Research gap \\ None}

\section{References}

1. Birgfeld CB, Heike C. Craniofacial microsomia. Semin Plast Surg. 2012;26(2):91-104.

2. Horgan JE, Padwa BL, LaBrie RA, Mulliken JB. OMENSPlus: analysis of craniofacial and extracraniofacial anomalies in hemifacial microsomia. Cleft Palate Craniofac J. 1995;32(5):405-12.

3. Vento AR, LaBrie RA, Mulliken JB. The O.M.E.N.S. classification of hemifacial microsomia. Cleft Palate Craniofac J. 1991;28(1):68-76; discussion 7.

4. Tasse C, Bohringer S, Fischer S, Ludecke HJ, Albrecht B, Horn D, et al. Oculo-auriculo-vertebral spectrum (OAVS): clinical evaluation and severity scoring of 53 patients and proposal for a new classification. Eur J Med Genet. 2005;48(4):397-411.

5. Pruzansky S. Not all dwarfed mandibles are alike. Birth defects: Original Article Series. 1969;5:120-9.

6. Converse JM, Wood-Smith D, McCarthy JG, Coccaro PJ, Becker MH. Bilateral facial microsomia. Diagnosis, classification, treatment. Plast Reconstr Surg. 1974;54(4): 413-23.

7. David DJ, Mahatumarat C, Cooter RD. Hemifacial microsomia: a multisystem classification. Plast Reconstr Surg. 1987;80(4):525-35.

8. Lauritzen C, Munro IR, Ross RB. Classification and treatment of hemifacial microsomia. Scand J Plast Reconstr Surg. 1985;19(1):33-9.

9. Birgfeld CB, Luquetti DV, Gougoutas AJ, Bartlett SP, Low DW, Sie KC, et al. A phenotypic assessment tool for craniofacial microsomia. Plast Reconstr Surg. 2011;127(1): 313-20.
10. Kaban LB, Moses MH, Mulliken JB. Correction of hemifacial microsomia in the growing child: a follow-up study. Cleft Palate J. 1986;23 Suppl 1:50-2.

11. Kaban LB, Mulliken JB, Murray JE. Three-dimensional approach to analysis and treatment of hemifacial microsomia. Cleft Palate J. 1981;18(2):90-9.

12. Xu S, Zhang Z, Tang X, Yin L, Liu W, Shi L. The influence of gender and laterality on the incidence of hemifacial microsomia. J Craniofac Surg. 2015;26(2):384-7.

13. Bennun RD, Mulliken JB, Kaban LB, Murray JE. Microtia: a microform of hemifacial microsomia. Plast Reconstr Surg. 1985;76(6):859-65.

14. Rollnick BR, Kaye CI, Nagatoshi K, Hauck W, Martin AO. Oculoauriculovertebral dysplasia and variants: phenotypic characteristics of 294 patients. Am J Med Genet. 1987;26(2):361-75.

15. Rollnick BR, Kaye CI. Hemifacial microsomia and variants: pedigree data. Am J Med Genet. 1983;15(2):233-53.

16. Goldenhar M. Associations malformatives de l'oeil et de l'oreille, en particulier le syndrome dermoïde épibulbaire appendices auriculaires - fistula auris congenita et ses relations avec la dysostose mandibulofaciale. J Genet Hum. 1952; 1:243-82.

17. Gorlin RJ PJ. Syndromes of the head and neck. New York: McGraw-Hill. 1964:546-52.

18. Gorlin RJ, Jue KL, Jacobsen U, Goldschmidt E. Oculoauriculovertebral Dysplasia. J Pediatr. 1963;63:991-9.

19. Heike CL, Wallace E, Speltz ML, Siebold B, Werler MM, Hing AV, et al. Characterizing facial features in individuals with craniofacial microsomia: A systematic approach for clinical research. Birth Defects Res A Clin Mol Teratol. 2016;106(11):915-26.

20. Caron C, Pluijmers BI, Wolvius EB, Looman CWN, Bulstrode $\mathrm{N}$, Evans RD, et al. Craniofacial and extracraniofacial anomalies in craniofacial macrosomia: A multicenter study of 755 patients. J Craniomaxillofac Surg. 2017;45(8):1302-10.

21. Tuin AJ, Tahiri Y, Paine KM, Paliga JT, Taylor JA, Bartlett SP. Clarifying the relationships among the different features of the OMENS + classification in craniofacial microsomia. Plast Reconstr Surg. 2015;135(1):149e-56e.

22. Tuin J, Tahiri Y, Paliga JT, Taylor JA, Bartlett SP. Distinguishing Goldenhar Syndrome from Craniofacial Microsomia. J Craniofac Surg. 2015;26(6):1887-92.

23. Measurement ICfHO. ICHOM Craniofacial Microsomia Data Collection Reference Guide. 2017.

\section{CHAPTER 4. SCREENING, MONITORING AND INDICATION FOR TREATMENT}

\section{1 Breathing difficulties in craniofacial microsomia \\ Introduction}

Obstructive sleep disordered breathing (SDB) can be considered to be a syndrome of upper airway dysfunction during sleep (1). It is characterised by snoring and/or increased respiratory effort due to increased airway resistance and pharyngeal collapsibility $(2,3)$. Various clinical entities with intermittent upper airway obstruction are included in obstructive SDB, such as: primary snoring, upper airway resistance syndrome, obstructive hypoventilation, and obstructive sleep apnoea (OSA) (4, 5). Polysomnography (sleep study) is the golden standard to diagnose presence and severity of sleep disordered breathing (SDB). Of the obstructive SDB entities, OSA in particular is known to be of clinical significance as it relates 
to failure to thrive, morning headache, excessive daytime sleepiness, decline of school achievements, feeding difficulties, upper airway infections, hypertension, and may lead to metabolic, cognitive and cardiovascular consequences (6). OSA is defined by a disruption of the normal oxygenation, ventilation and sleep pattern due to recurrent upper airway obstructions. The severity of OSA is described by the Apnoea-Hypopnea Index (AHI).

The prevalence of OSA in children in the healthy population is $2-4 \%$, and is thought to be caused by adenotonsillar hypertrophy, obesity, or decrease in neuromuscular tone (7-10). Patients with craniofacial microsomia (CFM) are at increased risk for obstructive SDB and OSA due to the underdevelopment of the mandible, which leads to obstruction of the upper airway. In addition, pharyngeal and/or laryngeal anomalies may play a role in causing OSA in patients with CFM $(11,12)$. Since OSA in patients with CFM relates to congenital malformations, symptoms occur shortly after birth or during childhood.

The European Respiratory Society provided a stepwise approach to diagnose, manage and treat obstructive SDB and OSA in children $(1,13)$. Treatment of OSA in patients with major craniofacial abnormalities, such as CFM, is reported as a priority. Obstructive SDB, including OSA, is treated by a stepwise approach and can require pharmacological treatment such as nasal corticosteroids, non-surgical treatment by continuous positive airway pressure (CPAP) or non-invasive positive-pressure ventilation (NPPV), or surgical treatment such as adenotonsillectomy or craniofacial surgery. In addition to the regular treatment modalities, patients with CFM may require surgical correction of the mandible by using mandibular distraction, costochondral grafting or osteotomy (11).

To describe the policy for breathing difficulties in patients with CFM the following questions were included in this chapter:

\subsubsection{What is the type, prevalence and severity of breathing difficulties in craniofacial microsomia? \\ 4.1.2 What is the policy for screening and monitoring of breathing difficulties (OSA) in patients with craniofacial microsomia? \\ 4.1.3 What are the indications and policy for treatment of breathing difficulties (OSA) in patients with craniofacial microsomia?}

Describing the policy for breathing difficulties in patients with CFM is of importance since these patients are at increased risk for OSA. Untreated OSA may lead to metabolic, cognitive and/or cardiovascular pathology and can have a negative impact on the quality of life. Therefore, timely diagnosis and treatment of OSA in patients with CFM is considered of importance to patients.

\section{Definitions}

The definitions of apnoea's, hypopnea and OSA severity are displayed in Appendix 4, http://links.lww.com/SCS/B697. This is based on the Manual for the Scoring of Sleep and Associated Events of the American Academy of Sleep Medicine (14). The severity of OSA is graded according to the New Zealand Guideline for obstructive sleep apnoea syndrome in children (15).

\section{Literature search}

A systematic search of literature was performed to identify all available literature on CFM and synonyms. The search was conducted in Embase, Pubmed/Medline Ovid. The full search strategy is reported in the supplementary material.

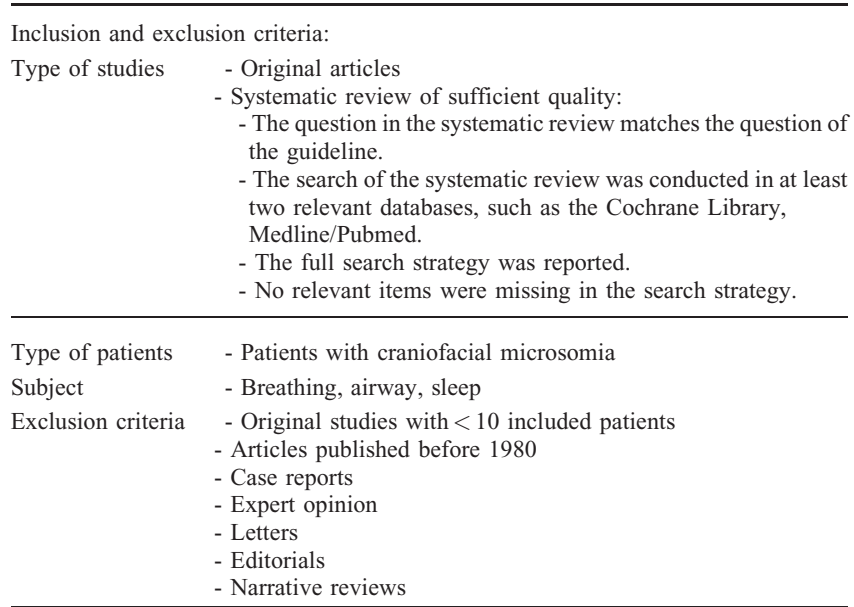

Literature was screened on title and abstract. A total of eight articles were included. One systematic review by Caron et al. was included (11). Five studies were retrospective cohort studies: Szpalski et al. (6), Cohen et al. (16), Sculerati et al. (17), Sher et al. (18), and Caron et al. (19). The other two included studies were a cross-sectional study by D'Antonio et al. (12) and a case-control study by Cloonan et al. (20).

4.1.1 What is the type, prevalence and severity of breathing difficulties in craniofacial microsomia?

The literature search for this question was directed towards more specific questions related to the type (primary snoring, upper airway resistance syndrome, obstructive hypoventilation, and OSA), prevalence and severity (mild, moderate or severe) of breathing difficulties in CFM (Appendix 1, http://links.lww.com/SCS/B697). Unfortunately, none of the included studies reported information on the type of breathing difficulties in CFM. Studies only reported on one of the types of breathing difficulties, OSA. Since this question does not relate to interventions or diagnostics, no considerations, rationale or recommendations are provided.

Review of literature

A systematic review on obstructive sleep apnoea by Caron et al. was published in 2015. The aim of the study was to report the prevalence and treatment modalities of OSA in patients with CFM. Six studies describing the prevalence of OSA in CFM were included in the systematic review. However, one study included fewer than ten patients and was not taken into account in this guideline (21). The prevalence of OSA in CFM varied from $7 \%$ to $24 \%(12,16-18,20)$. The number of patients included in these studies varied from 38 to 124 patients. Most studies were retrospective cohort studies and the diagnosis of OSA was based on chart review and polysomnography. Although some studies defined the used diagnostic criteria for $\operatorname{OSA}(16,18)$, other studies did not $(12,17,20)$. Sculerati et al. included 41 patients of which 9 patients required a tracheostomy (17). Therefore, the prevalence of OSA in this study, which can be considered to be severe, is found to be $22 \%$. However, no additional data is available on the presence of OSA in the other included patients. One of the other included studies analysed 124 patients with CFM and 349 controls (20). Patients with CFM had a higher frequency of SDB $(23 \%$ vs $9 \%$, OR: 3.0 , CI: $1.5-5.1$, $\mathrm{p}=0.001$ ), which includes OSA, compared to controls. None of the included studies reported information on the type of breathing difficulties in CFM. The severity of OSA and risk factors for OSA in patients with CFM were assessed by multiple studies. Cloonan et al. reported that patients with moderate/severe CFM presented more often with snoring and SDB than patients with mild CFM. In this study, the diagnosis mild, moderate or severe CFM was based on specialists' 
assessment of craniofacial anomalies. The prevalence of OSA was found to be significantly higher in patients with a more severe form of CFM, i.e. severe mandibular hypoplasia and/or bilateral involvement $(16,20)$. Bilateral hearing loss or a more severe orbital deformity was found to be a risk factor for OSA too (16).

Besides patients with bilateral CFM, patients with unilateral CFM may be at risk for OSA too. Szpalski et al. included 62 patients with unilateral CFM and retrospectively studied the prevalence of OSA and any significant associations (6). Polysomnography was only assessed in patients with signs or symptoms of OSA. The severity of OSA was scored as defined in this guideline. The reported prevalence of OSA in unilateral CFM was $11.3 \%$ (7 out of 62 patients). Severe OSA was found in three patients, moderate OSA in two patients, and two patients had mild OSA. Interestingly, all patients with OSA had a mandibular deformity Pruzansky IIB or higher. Of the six patients with unilateral CFM and a Pruzansky III deformity only one patient did not have OSA.

A large, multicentre, retrospective study on obstructive sleep apnoea in CFM was undertaken by Caron et al. in 2017 (19). This study aimed to report the prevalence of OSA in CFM, study the relationship of OSA and CFM severity and analyse treatment modalities for OSA in CFM. The severity of OSA in children was defined as follows: mild OSA, obstructive apnoea-hypopnea index (oAHI) 1-5; moderate OSA, oAHI 5-24; severe OSA, oAHI >24. In adults the criteria were: mild OSA, AHI 5-15; moderate OSA, AHI 15-30; severe OSA, AHI $>30$. Of the 755 patients included, a total of 133 were reported to have OSA. Thus, the prevalence of OSA in CFM was $17.6 \%$. OSA was diagnosed at a median age of 2.4 years (range $0-$ 25.8 years). Only five patients were adult patients ( $>18$ years) at the time of diagnosis. The severity of OSA was mild in 25 patients, moderate in 22 patients, severe in 48 patients, and in 38 patients the severity of OSA was unknown. Patients with bilateral CFM had a higher prevalence of OSA than unilateral patients. Patient with a Pruzansky IIB/III mandibular deformity, both uni- and bilateral, had a higher prevalence of OSA than patients with Pruzansky I or IIA. Severe OSA was thus found more frequently in patients with a Pruzansky IIB/III deformity. Of the patients with a Pruzansky III and OSA, OSA was severe in $52 \%$ of the cases.

Moraleda-Cibrián studied patients with various craniofacial malformations who underwent PSG (22). Of the 153 patients included, 18 patients were diagnosed with CFM. After PSG, one patient did not have OSA, 11 patients were diagnosed with mild OSA, 5 patients had moderate OSA and one patient had severe OSA. No further information on patient characteristics or specification of breathing difficulties of these patients was provided (Figure 1).

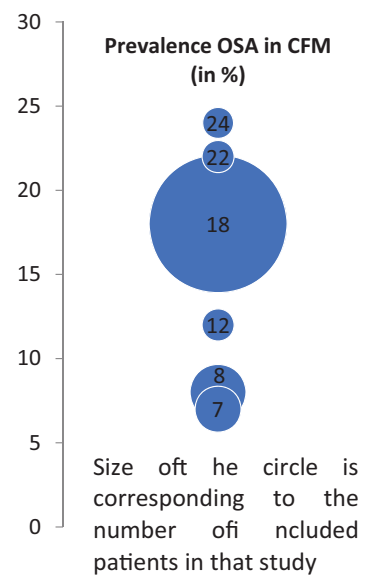

Figure 1. Prevalence of OSA in CFM relative to sample size.

\section{Conclusions}

Prevalence
$\begin{aligned} & \text { The prevalence of obstructive sleep apnoea in craniofacial microsomia } \\ & \text { is } 7-24 \% \text {, presumably approximating } 18 \% .\end{aligned}$
$\operatorname{Ref}(6,12,16-20)$

Level $3 \quad \begin{aligned} & \text { Prevalence } \\ & \text { Patients with bilateral craniofacial microsomia have a higher } \\ & \text { prevalence of obstructive sleep apnoea than patients with } \\ & \text { unilateral craniofacial microsomia. }\end{aligned}$
$\operatorname{Ref}(16,19)$

Level 3

Prevalence

Patients with a Pruzansky grade IIB or III mandibular deformity, both unilateral or bilateral, have a higher prevalence of obstructive sleep apnoea compared to patients with a Pruzansky grade I or IIA.

$\operatorname{Ref}(6,16,19)$

\begin{tabular}{l} 
Level $32 \begin{array}{c}\text { Prevalence } \\
\text { The prevalence of severe obstructive sleep apnoea is higher in } \\
\text { patients with a Pruzansky grade IIB or III mandibular } \\
\text { deformity. } \\
\operatorname{Ref}(6,19)\end{array}$ \\
\hline
\end{tabular}

\begin{tabular}{l} 
Level $3 \quad \begin{array}{c}\text { Severity } \\
\text { The severity of obstructive sleep apnoea was mild in } 19-29 \% \text { of } \\
\text { cases, moderate in } 17-29 \% \text {, severe in } 36-43 \% \text {, and in } 29 \% \\
\text { the severity of obstructive sleep apnoea was unknown. } \\
\operatorname{Ref}(6,19)\end{array}$ \\
\hline
\end{tabular}

4.1.2 What is the policy for screening and monitoring of breathing difficulties (OSA) in patients with craniofacial microsomia?

This question was focussed on OSA since none of the included studies reported information on other types of breathing difficulties in CFM. Additionally, OSA is a diagnosis that is confirmed with objective measures and is important to diagnose and/or treat due to its clinical significance and potential consequences. The literature search for this question was directed towards more specific questions related to the consequences and impact of screening and monitoring, the available screening tests and procedures for monitoring, and responsibilities for screening and monitoring (Appendix 1, http://links.lww.com/SCS/B697). Unfortunately, no literature is available on the policy for screening and/ or monitoring of OSA in patients with CFM. Therefore no conclusions were written. Considerations for this question were only based on expert opinion.

\section{Considerations \\ - Overall quality of evidence}

The overall quality of evidence is low because there is no literature available and therefore the recommendations are based on expert opinion with a low quality of evidence.

\section{- Balance of benefits and harms}

Due to the high prevalence of breathing disorders all patients with CFM should be screened for OSA. Patients who have Pruzansky-Kaban IIb or III mandibles and/or are bilaterally affected should undergo a polysomnography because of the high risk of developing OSA. Because OSA is associated with adverse outcome, the benefits of screening outweigh the harms. 


\section{- Outcome importance}

Untreated OSA may have severe consequences in growth, daily functioning and (cognitive) development. Therefore, screening and monitoring at regular times is necessary.

\section{- Costs and resources}

The impact on costs and resources of the given recommendations will vary per member state, depending on the available care providers and facilities. The recommendations concern the essential requirements for adequate treatment of patients with CFM and should thus be implemented. Costs are lowest and resources are most efficiently used when care for craniofacial disorders is centralised in a limited number of expert centres per member state. A general rule that can be applied is one expert centre per 10 million inhabitants.

\section{- Inequity of the recommendation}

The goal of the European Reference Networks (ERNs) is to eliminate inequality within Europe with regard to care for patients with rare diseases. At present, not every member state offers an expert centre for CFM, or the level of provided care does not (yet) meet all the requirements that are outlined in this guideline. By defining the baseline of required care for CFM, this guideline will help these member states to reach the appropriate level. The ERN on craniofacial anomalies and ENT disorders (ERN-CRANIO) can guide a patient in Europe to the available centres of expertise (www.ern-cranio.eu) and can support care providers with diagnosis and treatment advice.

\section{- Feasibility of the recommendation}

Recommendations refer to the general requirements for delivering optimal healthcare and are discussed with members from participating European countries. Quality of care was paramount in the discussions. Centralisation is proposed as one of the core values. However, in some countries the national organisation of healthcare might impede centralisation. National implementation of the ERNs that fits the situation of each country is necessary. For the member states with the lowest number of inhabitants, the establishment of a craniofacial centre might not be feasible, and collaboration with a craniofacial centre in the surrounding countries can be an option.

\section{- Acceptability of the recommendation}

It is expected that all stakeholders want to apply and will be applying the recommendations, because they are employed in ERNacknowledged institutions. National implementation plans are necessary to ensure that recommendations fit the situation in each country. In addition, not all countries participating in the ERNCRANIO are represented in the guideline development group, and new members will join within the coming years. For these countries, acceptance and implementation of ERN guidelines such as this guideline on CFM is obligatory.

\section{Rationale of the recommendations}

Undiagnosed or untreated OSA has been associated with learning impairment and behavioural problems such as daytime hyperactivity, aggression or attention deficit hyperactivity disorder (ADHD). More serious complications include neurologic and developmental delay, failure to thrive, cardiovascular disease, including pulmonary hypertension and right ventricular hypertrophy, and sudden death. Patients with OSA are at increased risk for feeding difficulties (23). Several authors, including the European Respiratory Society, have emphasised early and accurate diagnosis of OSA by routinely screening patients who are at higher risk for OSA such as patients with CFM. Biannual screening should be performed by using the Paediatric Sleep Questionnaire (PSQ), which is agreed on in the ICHOM Standard Set for Craniofacial Microsomia (24). Screening should be performed at least up to the age of six in the outpatient department. This questionnaire is free for all healthcare organisations and a license is not needed.

Recommendations

- All patients with craniofacial microsomia should be screened with a questionnaire biannually, at least up to the age of six, in the outpatient department for a clinical history of obstructive sleep apnoea.

- If there is a suspicion of obstructive sleep apnoea based on a questionnaire, a polysomnography (sleep study) has to be performed.

- All patients who have Pruzansky-Kaban IIb or III mandibles and/or are bilaterally affected have to undergo a polysomnography (sleep study) to screen for obstructive sleep apnoea in the first year of life.

\section{Research gap}

Studies on the consequences of mild, moderate or severe OSA in patients with CFM are not available. Future studies on detailed assessment of PSG's and the consequences of OSA in CFM are needed.

4.1.3 What are the indications and policy for treatment of breathing difficulties (OSA) in patients with craniofacial microsomia?

The literature search for this question was directed towards more specific research questions related to treatment options, symptoms, requirements, complications, and advantages and disadvantages of treatment of breathing difficulties (Appendix 1, http://links.lww.$\mathrm{com} / \mathrm{SCS} / \mathrm{B} 697)$. Unfortunately, no literature on the indications to treat OSA based on its severity in patients with CFM is present. However, descriptive studies on treatment modalities used for OSA in patients with CFM are available. These studies may provide more insight on the indications and policy for treatment of OSA in patients with CFM and are thus included in this chapter. Therefore, conclusions were only written for the policy for treatment of breathing difficulties. Considerations for these questions were based on both literature and expert opinion.

\section{Review of literature}

A systematic review by Caron et al. included twelve studies involving treatment modalities for OSA in CFM (11). Most of these studies were case reports or case series including fewer than ten patients. Two of the included studies were included in this guideline $(17,18)$. Sculerati et al. studied 41 patients, of which nine needed tracheostomy (17). No additional information on follow-up treatment of OSA was provided. In the study by Sher et al., 84 patients with CFM were included; six patients were diagnosed with OSA (18). All patients with OSA underwent nasopharyngoscopic examination of the upper airway. In three patients there was a circular constriction of the pharynx. Two of these patients required tracheotomy and one patient refused treatment. Obstruction of the upper airway due to posterior movement of the tongue to the posterior pharyngeal wall was observed in two patients. Both patients had a mandibular advancement. The last patient also had an obstruction of the upper airway due to movement of the tongue, but in this case the tongue compressed the soft palate 
causing closure in the oropharynx. This patient also required tracheostomy. Caron et al. concluded that good literature on non-surgical and/or surgical treatment modalities for OSA in CFM is not available (11). Due to the lack of information no ideal treatment plan for OSA in patients with CFM could be proposed.

One of the aims of the retrospective study by Caron et al. was to analyse the treatment modalities of OSA and their outcomes in patients with CFM (19). Of the 133 patients with OSA included, 102 required treatment, 20 patients were not treated, and of 11 patients the treatment status was unknown. Different treatment modalities were chosen for initial treatment: adenotonsillectomy (ATE) (47\%), tracheostomy (31\%), continuous positive airway pressure (CPAP) (13\%), placement of a nasal pharyngeal airway (4\%), prone positioning (1\%), mandibular distraction osteogenesis (MDO) $(1 \%)$, or other surgical procedures such as nasal septum correction (3\%). In 84 of the 102 patients $(80 \%)$ requiring treatment, a surgical procedure such as ATE, tracheostomy or MDO was treatment of first choice. Of the 48 patients receiving ATE as initial treatment for OSA, 41 patients $(85 \%)$ did not receive additional treatment. Thirty-two patients did not have a post-treatment PSG, in eight patients the post-treatment PSG was normal and in one patient the ATE had no effect on the severity of OSA. Of the patients treated by ATE, seven patients did receive additional treatment: CPAP in three patients, placement of a nasal pharyngeal airway in two patients, one patient had a tracheotomy and one patient had other therapy not specified. ATE was the initial therapy for most patients with mild and moderate OSA but was applied in patients with severe OSA too. Tracheostomy was the initial therapy in 32 patients and the first choice of treatment for $67 \%$ of the patients with severe OSA. Eighteen patients received additional treatment, nine patients with the tracheostomy still in situ and nine patients without tracheostomy. Of all patients with a tracheostomy, $51.4 \%$ were decannulated or their posttreatment PSG was normal. CPAP was the first choice of treatment in thirteen patients $(13 \%)$ and the secondary choice of treatment in thirteen patients too. After additional therapy with CPAP, $46.2 \%$ of the patients needed additional therapy. Although eleven patients (unilateral) were treated by mandibular distraction osteogenesis, it was the initial therapy in only one patient. The outcome of MDO on OSA was good in four patients, having a normal post-treatment PSG. In five patients no post-treatment PSG was performed. Two patients still required CPAP and one patient had a persistent tracheostomy.

Conclusions

Level $3 \quad$ Treatment modalities
Treatment modalities for obstructive sleep apnoea in craniofacial
microsomia are: prone positioning, nasal pharyngeal airway
tube, continuous positive airway pressure (CPAP),
adenotonsillectomy (ATE), tracheostomy, mandibular
distraction osteogenesis, and mandibular osteotomy.
$\operatorname{Ref}(11,18,19)$

\begin{tabular}{l}
\hline Level 3 Policy \\
In patients with mild to severe obstructive sleep apnoea and \\
adenotonsillar hypertrophy, adenotonsillectomy (ATE) may be the \\
treatment of first choice. Most patients do not require additional \\
therapy for their obstructive sleep apnoea. \\
Continuous positive airway pressure (CPAP) appears to be a good \\
treatment for moderate to severe obstructive sleep apnoea in \\
craniofacial microsomia. \\
In patients with severe obstructive sleep apnoea, tracheostomy is often \\
considered the first choice of treatment. \\
Mandibular distraction osteogenesis (MDO) is often applied as \\
secondary treatment for obstructive sleep apnoea caused by \\
obstruction at the tongue base in craniofacial microsomia. \\
Additional therapy after MDO is indicated in some patients. \\
Ref (19)
\end{tabular}

\section{Considerations \\ - Overall quality of evidence}

The treatment modalities for OSA in CFM were described in two retrospective studies $(18,19)$ and the systematic review by Caron et al (11). The studies are descriptive retrospective cohort studies and were therefore graded on level 3 .

The choice of treatment for OSA in CFM was analysed in the retrospective study by Caron et al (19). Treatment modalities and outcomes were retrospectively studied and no controls were used. The quality of evidence was graded on level 3.

\section{- Balance of benefits and harms}

Untreated OSA may lead to serious morbidity and worse outcomes. Treatment of OSA will be beneficial for the child suffering from OSA and outweigh the harms.

\section{- Outcome importance}

Untreated OSA will impair outcome, specially growth and development. Therefore OSA has to be treated.

\section{Costs and resources}

The impact on costs and resources of the given recommendations will vary per member state, depending on the available care providers and facilities. The recommendations concern the essential requirements for adequate treatment of patients with CFM and should thus be implemented. Costs are lowest and resources are most efficiently used when care for craniofacial disorders is centralised in a limited number of expert centres per member state. A general rule that can be applied is one expert centre per 10 million inhabitants.

\section{- Inequity of the recommendation}

The goal of the European Reference Networks (ERNs) is to eliminate inequality within Europe with regard to care for patients with rare diseases. At present, not every member state offers an expert centre for CFM, or the level of provided care does not (yet) meet all the requirements that are outlined in this guideline. By defining the baseline of required care for CFM, this guideline will help these member states to reach the appropriate level. The ERN on craniofacial anomalies and ENT disorders (ERN-CRANIO) can guide a patient in Europe to the available centres of expertise (www.ern-cranio.eu) and can support care providers with diagnosis and treatment advice.

\section{- Feasibility of the recommendation}

Recommendations refer to the general requirements for delivering optimal healthcare and are discussed with members from participating European countries. Quality of care was paramount in the discussions. Centralisation is proposed as one of the core values. However, in some countries the national organisation of healthcare might impede centralisation. National implementation of the ERNs that fits the situation of each country is necessary. For the member states with the lowest number of inhabitants, the establishment of a craniofacial centre might not be feasible, and collaboration with a craniofacial centre in the surrounding countries can be an option.

\section{- Acceptability of the recommendation}

It is expected that all stakeholders want to apply and will be applying the recommendations, because they are employed in ERNacknowledged institutions. National implementation plans are necessary to ensure that recommendations fit the situation in each country. In addition, not all countries participating in the ERN- 
CRANIO are represented in the guideline development group, and new members will join within the coming years. For these countries, acceptance and implementation of ERN guidelines such as this guideline on CFM is obligatory.

\section{Rationale of the recommendations}

Untreated OSA has been associated with major impairments to growth and (cognitive) development and may lead to serious complications. Several authors, including the European Respiratory Society, have emphasised the need to treat OSA. There is no uniform protocol or treatment algorithm available for treatment of OSA in children with CFM. Non-surgical treatment for breathing difficulties should always be considered in children with OSA. Treatment modalities depend on the experience of a centre, the age of the child, the possibility to perform an endoscopy to detect the level of obstruction and the severity of symptoms. Therefore, because of the complexity of the treatment, a multidisciplinary team has to discuss clinical findings and results of additional investigations to decide on the appropriate treatment. As described in the literature, MDO should be considered to prevent a tracheostomy or aim for decannulation in patients with severe OSA.

\section{Recommendations}

- Treatment of children with craniofacial microsomia and obstructive sleep apnoea has to be discussed in a multidisciplinary team.

- Treatment of children with craniofacial microsomia and obstructive sleep apnoea depends on the age of the child, the severity of symptoms and the level of obstruction.

- In older children with mild to severe obstructive sleep apnoea, adenotonsillectomy (ATE) may be the treatment of first choice.

- In young infants and children with craniofacial microsomia and obstructive sleep apnoea non-surgical respiratory support has to be considered to treat obstructive sleep apnoea.

- In children with craniofacial microsomia and severe obstructive sleep apnoea a tracheostomy has to be considered at all ages.

- Mandibular distraction osteogenesis (MDO) should be considered to treat patients with severe obstructive sleep apnoea who have a tracheostomy or to reduce the necessity for a tracheostomy or respiratory support.

\section{Research gap}

The literature on treatment modalities for OSA in CFM is retrospective and no comparative studies have been done. The preferred type of treatment for mild, moderate or severe OSA in CFM has not been studied. Future studies on this subject, with longterm follow-up and patient-specific outcomes, are needed.

\section{References}

1. Kaditis AG, Alonso Alvarez ML, Boudewyns A, Alexopoulos EI, Ersu R, Joosten K, et al. Obstructive sleep disordered breathing in 2- to 18-year-old children: diagnosis and management. Eur Respir J. 2016;47(1):69-94.

2. Kaditis A, Kheirandish-Gozal L, Gozal D. Algorithm for the diagnosis and treatment of pediatric OSA: a proposal of two pediatric sleep centers. Sleep Med. 2012;13(3):217-27.

3. Katz ES, D'Ambrosio CM. Pathophysiology of pediatric obstructive sleep apnea. Proc Am Thorac Soc. 2008;5(2):25362 .

4. Standards and indications for cardiopulmonary sleep studies in children. American Thoracic Society. Am J Respir Crit Care Med. 1996;153(2):866-78.

5. Dayyat E, Kheirandish-Gozal L, Gozal D. Childhood Obstructive Sleep Apnea: One or Two Distinct Disease Entities? Sleep Med Clin. 2007;2(3):433-44.

6. Szpalski C, Vandegrift M, Patel PA, Appelboom G, Fisher M, Marcus J, et al. Unilateral Craniofacial Microsomia: Unrecognized Cause of Pediatric Obstructive Sleep Apnea. J Craniofac Surg. 2015;26(4):1277-82.

7. Anuntaseree W, Kuasirikul S, Suntornlohanakul S. Natural history of snoring and obstructive sleep apnea in Thai schoolage children. Pediatr Pulmonol. 2005;39(5):415-20.

8. Bixler EO, Vgontzas AN, Lin HM, Liao D, Calhoun S, VelaBueno A, et al. Sleep disordered breathing in children in a general population sample: prevalence and risk factors. Sleep. 2009;32(6):731-6.

9. Lumeng JC, Chervin RD. Epidemiology of pediatric obstructive sleep apnea. Proc Am Thorac Soc. 2008;5(2): 242-52.

10. Ishman SL. Evidence-based practice: pediatric obstructive sleep apnea. Otolaryngol Clin North Am. 2012;45(5):1055-69.

11. Caron CJJM, Pluijmers BI, Joosten KFM, Mathijssen IMJ, Van Der Schroeff MP, Dunaway DJ, et al. Obstructive sleep apnoea in craniofacial microsomia: A systematic review. Int J Oral Maxillofac Surg. 2015;44(5):592-8.

12. D'Antonio LL, Rice Jr RD, Fink SC. Evaluation of pharyngeal and laryngeal structure and function in patients with oculoauriculo-vertebral spectrum. Cleft Palate-Craniofac J. 1998;35(4):333-41.

13. Kaditis AG, Alonso Alvarez ML, Boudewyns A, Abel F, Alexopoulos EI, Ersu R, et al. ERS statement on obstructive sleep disordered breathing in 1- to 23-month-old children. Eur Respir J. 2017;50(6).

14. Berry RB, Budhiraja R, Gottlieb DJ, Gozal D, Iber C, Kapur $\mathrm{VK}$, et al. Rules for scoring respiratory events in sleep: update of the 2007 AASM Manual for the Scoring of Sleep and Associated Events. Deliberations of the Sleep Apnea Definitions Task Force of the American Academy of Sleep Medicine. J Clin Sleep Med. 2012;8(5):597-619.

15. New Zealand Guidelines Group Obstructive sleep apnea syndrome in children. 2006.

16. Cohen SR, Levitt CA, Simms C, Burstein FD. Airway disorders in hemifacial microsomia. Plast Reconstr Surg. 1999;103(1):27-33.

17. Sculerati N, Gottlieb MD, Zimbler MS, Chibbaro PD, McCarthy JG. Airway management in children with major 
craniofacial anomalies. Laryngoscope. 1998;108(12):180612.

18. Sher AE, Shprintzen RJ, Thorpy MJ. Endoscopic observations of obstructive sleep apnea in children with anomalous upper airways: predictive and therapeutic value. Int $\mathrm{J}$ Pediatr Otorhinolaryngol. 1986;11(2):135-46.

19. Caron CJJM, Pluijmers BI, Maas BDPJ, Klazen YP, Katz ES, Abel F, et al. Obstructive sleep apnoea in craniofacial microsomia: analysis of 755 patients. Int J Oral Maxillofac Surg. 2017;46(10):1330-7.

20. Cloonan YK, Kifle Y, Davis S, Speltz ML, Werler MM, Starr JR. Sleep outcomes in children with hemifacial microsomia and controls: A follow-up study. Pediatrics. 2009;124(2): e313-e21.

21. Luna-Paredes C, Anton-Pacheco JL, Garcia Hernandez G, Martinez Gimeno A, Romance Garcia AI, Garcia R, II. Screening for symptoms of obstructive sleep apnea in children with severe craniofacial anomalies: assessment in a multidisciplinary unit. Int J Pediatr Otorhinolaryngol. 2012;76(12): 1767-1770.

22. Moraleda-Cibrián M, Edwards SP, Kasten SJ, Buchman SR, Berger M, O'Brien LM. Obstructive sleep apnea pretreatment and posttreatment in symptomatic children with congenital craniofacial malformations. J Clin Sleep Med. 2015;11(1):3743.

23. Caron C, Pluijmers BI, Joosten KFM, Dunaway D, Padwa BL, Wolvius EB, et al. Feeding difficulties in craniofacial microsomia: A multicenter retrospective analysis of 755 patients. J Craniomaxillofac Surg. 2018;46(10):1777-82.

24. Measurement ICHOM. ICHOM Craniofacial Microsomia Data Collection Reference Guide. 2017.

\section{CHAPTER 4. SCREENING, MONITORING AND INDICATION FOR TREATMENT}

\subsection{Feeding difficulties in craniofacial microsomia \\ Introduction}

Besides aesthetic malformations, patients with craniofacial microsomia (CFM) also have functional difficulties. Characteristic features of patients with CFM, such as mandibular hypoplasia, facial nerve and/or masticatory muscle weakness, or anomalies of the oropharynx and larynx may all play a contributing factor in the feeding difficulties. Feeding difficulties may relate to difficulties in suckling, mastication, dysphagia, and/or failure to thrive. In 15$22 \%$ of the cases a cleft lip/palate is diagnosed in patients with CFM, which may further enhance the risk for feeding difficulties $(1,2)$.

Potential factors that may cause feeding difficulties in patients with CFM were discussed in a systematic review on this issue (3). Mandibular hypoplasia may cause a restricted mouth opening and limited sucking efficiency, causing feeding difficulties. Facial nerve weakness and/or - musculature hypoplasia may lead to limited cheek and lip movements, causing sucking and feeding difficulties. Also, anomalies of the tongue, oropharynx, and/or larynx may cause feeding difficulties in patients with CFM. Besides the facial malformations, extracraniofacial anomalies such as cardiac- or gastrointestinal anomalies may also play an important role in the presence of feeding difficulties.

To describe the policy for feeding difficulties in patients with craniofacial microsomia the following questions were included in this chapter:

\subsubsection{What is the type, prevalence and severity of feeding difficulties in craniofacial microsomia?}

4.2.2 What is the policy for screening and monitoring of feeding difficulties in patients with craniofacial microsomia? 4.2.3 What are the indications and policy for treatment of feeding difficulties in patients with craniofacial microsomia?

Diagnosis and treatment of feeding difficulties in an early phase is essential to prevent further harm. Especially in children with CFM, who may be at increased risk for these difficulties, vigilance for feeding difficulties is essential.

Literature search

A systematic search of literature was performed to identify all available literature on craniofacial microsomia and synonyms. The search was conducted in Embase, Pubmed/Medline Ovid. The full search strategy is reported in the supplementary material.

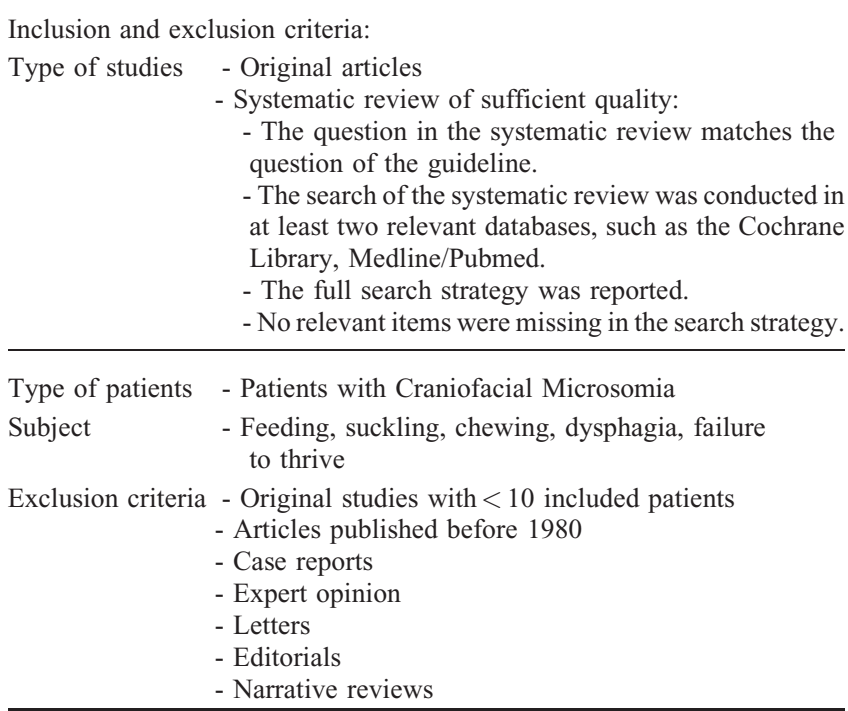

Literature was screened on title and abstract. Six articles were included in this chapter. A systematic review by Caron et al. (3) and cross-sectional study by Cohen et al. (4) were included. The other four articles were al retrospective cohort studies: Strömland et al. (5), Caron et al. (6), Van de Lande et al. (7), and Brotto et al. (8).

4.2.1 What is the type, prevalence and severity of feeding difficulties in craniofacial microsomia?

The literature search for this question was directed towards more specific questions related to the type (suckling, mastication, dysphagia, and/or failure to thrive), prevalence and severity (mild, moderate or severe) of feeding difficulties in CFM (Appendix 1, http://links.lww.com/SCS/B697). Since this question is not relate to interventions or diagnostics, no considerations, rationale or recommendations are provided.

\section{Review of literature \\ Type and prevalence}

In 2015 a systematic review of literature was undertaken by Caron et al. to provide an overview of literature on the prevalence and treatment of feeding difficulties in patients with CFM (3). A total of eight articles were included in this review. Six of the eight articles were excluded in this chapter due to inclusion of less than ten patients or publication before 1980 . The other studies included 24 and 18 patients, respectively $(4,5)$. Of the 24 patients studied by 
Cohen et al. (4), ten patients (42\%) had feeding difficulties. The type of feeding difficulties were: swallowing dysfunction, feeding difficulties not specified and/or failure to thrive. Of the patients with feeding difficulties, three patients had bilateral CFM, seven patients had unilateral CFM, and nine patients had extracraniofacial anomalies. In addition, nine of the ten patients with feeding difficulties had orofacial hypotonia, which was associated with poor expressive language skills. A similar outcome was found by Strömland et al. (5). Of the eighteen patients included, twelve patients (63\%) had feeding difficulties. Tube feeding was indicated in all patients. Three patients $(25 \%)$ required gastrostomy. It is unclear whether the nine patients who were tube fed were also able to feed orally. Dysphagia was reported in nine patients of the twelve patients with feeding difficulties. The type of feeding difficulties in the other three patients is unknown, although six patients complained of drooling. Of the nine patients with dysphagia, seven patients had impaired speech. All patients with cleft palate $(n=5)$ had feeding difficulties. The authors state that dysphagia may be the result of oral motor impairment and orofacial dysmorphology, but cardiac, gastrointestinal, or breathing difficulties may also cause feeding difficulties (5).

\section{Severity}

A large retrospective multicentre study to analyse feeding difficulties in CFM was performed by Caron et al. (6). The authors graded the severity of feeding difficulties based on type of treatment and scored as 'mild', 'moderate', or 'severe':

1. Mild feeding difficulties: patients able to feed pureed or solid foods orally regardless of minor adjustments such as the use of a bottle or nipple.

2. Moderate feeding difficulties: patients able to feed orally, but also dependent on additional tube feeding.

3. Severe feeding difficulties: patients dependent on tube feedings, i.e. nasopharyngeal, percutaneous, or parenteral.

A total of 755 patients with CFM were included. Feeding difficulties were present in $26 \%$ of the patients $(n=199)$ : 92 patients $(46 \%)$ with mild, 25 patients $(13 \%)$ with moderate, and 82 patients $(41 \%)$ with severe feeding difficulties. Feeding difficulties were diagnosed before the age of six months in $60 \%$ of the patients. Patients presented with difficulties in suckling, swallowing, chewing, complaints of reflux and/or a restricted mouth opening. Of the 120 patients with a cleft lip/ palate, 62 patients had feeding difficulties. Patients with obstructive sleep apnoea (OSA) were significantly more frequently diagnosed with feeding difficulties compared to non-OSA patients (Pearson's $\left.\chi^{2}(\mathrm{df} 1)=47.084 ; \mathrm{p}<0.001\right)$. The authors studied the risk factors for feeding difficulties and found that patients with cleft lip/palate, bilateral CFM, extracraniofacial anomalies such as heart or gastrointestinal anomalies, obstructive sleep apnoea, and/or more severe mandibular hypoplasia had a higher risk for feeding difficulties. Of the patients with bilateral CFM, $49 \%$ had feeding difficulties, $50 \%$ of the patients with extracraniofacial anomalies had feeding difficulties, and $60 \%$ of the patients with obstructive sleep apnoea had feeding difficulties. Patients with macrostomia, which is part of the CFM 'spectrum', did not have a higher risk for feeding difficulties compared to patients without macrostomia. Patient-specific outcomes of feeding difficulties, such as growth or developmental delay, have not been assessed in this study. The authors conclude that all patients with CFM should be screened for feeding difficulties by a speech and language therapist before the age of one. After the age of one, patients should be monitored by a feeding specialist on a regular basis (6).

\section{Swallowing difficulties}

Feeding difficulties may be the result of swallowing difficulties. To study swallowing difficulties and their incidence in patients with CFM, videofluoroscopic swallowing studies were evaluated by Van de Lande et al. (7). A total of 755 patients with CFM were included. Feeding difficulties were present in 208 patients (27.5\%) and 102 patients $(13.5 \%)$ had swallowing difficulties. A total of 42 patients with swallowing difficulties who underwent videofluoroscopic swallowing studies were analysed. swallowing studies were separated in an oral and pharyngeal phase. Patients younger than six months $(n=11)$ showed difficulties in all phases, but especially nasopharyngeal reflux (75\%), bolus formation (62.5\%), and aspiration $(62.5 \%)$ were seen frequently. Patients older than six months $(\mathrm{n}=31)$ mostly had difficulties in bolus formation $(55 \%)$, delayed/ variable swallow trigger (47.4\%), and post-swallow stasis (47.1\%). Aspiration occurred less frequently in patients older than six months $(23.8 \%)$. Patients with a more severe mandibular deformity, Pruzansky-Kaban III malformation, had difficulties in appropriate bolus formation significantly more often. In the pharyngeal phase, no difference was found between more and less severely affected patients. No difference in outcome of the videofluoroscopic swallowing studies was seen between unilateral and bilateral CFM patients. The authors recommend screening for swallowing difficulties by a speech and language therapist in all patients with CFM. In addition, patients who have a Pruzansky-Kaban III mandibular deformity or are at high risk for swallowing difficulties should be screened using videofluoroscopic swallowing studies (7).

\section{Hypoplasia and aplasia}

Saliva is required for adequate swallowing function and digestion. Loss of salivary flow may therefore contribute to feeding difficulties. Brotto et al. studied the rate of parotid and submandibular gland abnormalities in CFM (8). They found that 21 of their 25 included patients had hypoplasia or aplasia of the parotid gland. Aplasia was reported in eight patients: three patients had bilateral aplasia and five patients unilateral aplasia of the parotid gland. Of the 21 patients with parotid gland hypoplasia/aplasia, six patients had submandibular salivary gland hypoplasia too. No patient had isolated hypoplasia of the submandibular salivary gland. A significant association was found between parotid gland hypoplasia/aplasia and ipsilateral trigeminal and/or facial nerve abnormalities. Although most patients had hypoplasia/aplasia of the salivary glands, no patients had complaints of decreased saliva production. Besides salivation deficits, other factors such as jaw deformities and oral apraxia presumably play a more significant role in feeding difficulties (8).

\section{Conclusions}

Prevalence

The prevalence of feeding difficulties in craniofacial microsomia varies from $26 \%$ to $63 \%$, presumably approximating $26 \%$. $\operatorname{Ref}(3-7)$

\section{Level 3 Prevalence}

Patients with craniofacial microsomia and obstructive sleep apnoea are at increased risk for feeding difficulties (studied in 755 patients).

$\operatorname{Ref}(6)$

Level 3 Type

Type of feeding difficulties encountered in craniofacial microsomia are: difficulties in suckling, swallowing, chewing, complains of reflux, a restricted mouth opening, failure to thrive. $\operatorname{Ref}(4-6)$ 


\begin{tabular}{l}
\hline Level $3 \quad$ Incidence \\
The incidence of feeding difficulties is higher in craniofacial \\
microsomia patients with: \\
- Bilateral craniofacial microsomia \\
- Cleft lip/palate \\
- Cardiac or gastrointestinal anomalies \\
- Obstructive sleep apnoea \\
- Severe mandibular hypoplasia, i.e. Pruzansky-Kaban III \\
- Orofacial hypotonia \\
Ref (4-7)
\end{tabular}

\section{- Outcome importance}

In none of the studies the indication for treatment, treatment outcome and follow-up were described; however, this should be of the utmost importance in children because feeding difficulties can lead to impaired growth and development.

\section{- Costs and resources}

The impact on costs and resources of the given recommendations will vary per member state, depending on the available care providers and facilities. The recommendations concern the essential requirements for adequate treatment of patients with CFM and should thus be implemented. Costs are lowest and resources are most efficiently used when care for craniofacial disorders is centralised in a limited number of expert centres per member state. A general rule that can be applied is one expert centre per 10 million inhabitants.

\section{- Inequity of the recommendation}

The goal of the European Reference Networks (ERNs) is to eliminate inequality within Europe with regard to care for patients with rare diseases. At present, not every member state offers an expert centre for CFM, or the level of provided care does not (yet) meet all the requirements that are outlined in this guideline. By defining the baseline of required care for CFM, this guideline will help these member states to reach the appropriate level. The ERN on craniofacial anomalies and ENT disorders (ERN-CRANIO) can guide a patient in Europe to the available centres of expertise (www.ern-cranio.eu) and can support care providers with diagnosis and treatment advice.

\section{- Feasibility of the recommendation}

Recommendations refer to the general requirements for delivering optimal healthcare and are discussed with members from participating European countries. Quality of care was paramount in the discussions. Centralisation is proposed as one of the core values. However, in some countries the national organisation of healthcare might impede centralisation. National implementation of the ERNs that fits the situation of each country is necessary. For the member states with the lowest number of inhabitants, the establishment of a craniofacial centre might not be feasible, and collaboration with a craniofacial centre in the surrounding countries can be an option.

\section{- Acceptability of the recommendation}

It is expected that all stakeholders want to apply and will be applying the recommendations, because they are employed in ERNacknowledged institutions. National implementation plans are necessary to ensure that recommendations fit the situation in each country. In addition, not all countries participating in the ERNCRANIO are represented in the guideline development group, and new members will join within the coming years. For these countries, acceptance and implementation of ERN guidelines such as this guideline on CFM is obligatory.

\section{Rationale of the recommendations}

Considering the high prevalence of feeding difficulties in CFM and potential negative consequences of these difficulties, biannual screening for feeding difficulties is indicated in children with CFM. The role of the paediatrician, speech and language therapist and dietician is hardly described in existing research but should be standard care in children with feeding difficulties and growth failure. Paediatricians will screen for symptoms affecting the general condition of patients, growth and development. The WHO or national Growth Charts can be used to monitor growth 
and screen for feeding difficulties. In case of feeding difficulties, speech and language therapy should be the first step to improve oral-motor behaviour and swallowing difficulties. When children need tube feeding, a speech and language therapist has to be involved in follow-up of oral-motor behaviour. Therefore, close cooperation between the paediatrician and speech and language therapist is required at all times for the treatment of feeding difficulties in patients with CFM.

\section{Recommendations}

- Children with craniofacial microsomia should be screened with a questionnaire biannually, at least up to the age of six, and monitored regularly for feeding difficulties by a paediatrician or multidisciplinary team.

- The WHO or national Growth Charts can be used to monitor growth and screen for feeding difficulties.

- A speech and language therapist should be involved in patients who require tube feeding.

\section{Research gap}

No literature is available regarding the policy of screening and monitoring of feeding difficulties in patients with craniofacial microsomia. Availability of studies could help to develop better screening and monitoring.

4.2.3 What are the indications and policy for treatment of feeding difficulties in patients with craniofacial microsomia?

The literature search for this question was directed towards more specific research questions related to treatment options, symptoms, requirements, complications, and advantages and disadvantages of treatment of feeding difficulties (Appendix 1, http://links.lww.com/ $\mathrm{SCS} / \mathrm{B} 697)$. Literature was found on the indications of treatment options.

\section{Review of literature}

Treatment modalities for feeding difficulties in patients with CFM were further delineated in the retrospective study by Caron et al. (6). Table 1. shows the reported symptoms and treatment options in patients with mild, moderate, and severe feeding difficulties. Results were based on retrospective evaluation without comparison between treatment modalities based on patients specific outcomes, such as growth or developmental delay. Patients with mild feeding difficulties presented with chewing difficulties more frequently than with swallowing difficulties. Of the patients with moderate feeding difficulties, most had a Pruzansky-Kaban III mandibular deformity. Patients receiving tube feeding needed this for a median duration of 11.7 months (range 1.1 month -16.4 years). Patients with severe feeding difficulties were more frequently diagnosed with a Pruzansky-Kaban III mandibular deformity too. Four patients needed parenteral feeding, which was always followed by tube feedings. Tube feeding in patients with severe feeding difficulties was indicated for a median duration of 7.1 months (range 2 days -12.9 years). The authors discussed that in patients with bilateral CFM, and/or a severe mandibular deformity (i.e. Pruzansky-Kaban III), the presence of OSA, cleft lip/palate or extracraniofacial anomalies, surgical therapy such as cleft lip repair or mandibular distraction osteogenesis, may be indicated to treat feeding difficulties. In other patients, conservative treatment by using bottle/nipple feeding, supplemented (breast)milk, and/or antireflux medication, may be sufficient to treat feeding difficulties (6).

Table 2. Feeding difficulties in CFM (6)

\begin{tabular}{|c|c|c|c|}
\hline & $\begin{array}{l}\text { Mild* feeding } \\
\text { difficulties }(n=92)\end{array}$ & $\begin{array}{l}\text { Moderate* feeding }^{*} \\
\text { difficulties }(n=25)\end{array}$ & $\begin{array}{l}\text { Severe* feeding } \\
\text { difficulties }(n=82)\end{array}$ \\
\hline Cleft lip/palate & 16 patients & 8 patients & 38 patients \\
\hline $\mathrm{OSA}^{\#}$ & 24 patients & 14 patients & 42 patients \\
\hline Symptoms & $\begin{array}{l}\text { Swallowing difficulties } \\
\text { Suckling difficulties } \\
\text { Chewing difficulties } \\
\text { Restricted mouth opening }\end{array}$ & Not reported & $\begin{array}{l}\text { Difficulties in } \\
\text { swallowing } \\
\text { and/or reflux }\end{array}$ \\
\hline Treatment & $\begin{array}{l}\text { Bottle/nipple feeding } \\
\text { Pureed foods } \\
\text { Anti-reflux medication } \\
\text { Speech and } \\
\quad \text { language therapy }\end{array}$ & $\begin{array}{l}\text { Tube feeding } \\
\text { Gastrostomy }(\mathrm{n}=4) \\
\text { Adjustment of oral } \\
\text { feeding by same } \\
\text { therapy as patients } \\
\text { with mild feeding } \\
\text { difficulties }\end{array}$ & $\begin{array}{l}\text { Tube feeding } \\
\text { Parenteral } \\
\text { feeding }(\mathrm{n}=4) \\
\text { Gastrostomy } \\
\quad(\mathrm{n}=11)\end{array}$ \\
\hline
\end{tabular}

*based on the criteria applied by Caron et al (6). ${ }^{\#}$ OSA: obstructive sleep apnoea.

Conclusion

Level 3 Indications
Surgical treatment, such as cleft lip/palate repair or
mandibular distraction osteogenesis, may be indicated
for treatment of feeding difficulties in craniofacial
microsomia.
Conservative treatment, such as bottle/nipple feeding,
supplemented milk, or anti-reflux medication may be
sufficient in some patient for treatment of feeding
difficulties in craniofacial microsomia.
Ref (6)

\section{Considerations \\ - Overall quality of evidence}

Data on treatment modalities was based on a single retrospective study without comparison between treatment modalities based on patient specific outcomes (6). The indication for a certain type of treatment was not described in this study. Conclusions on CFMspecific factors of importance for a certain type of treatment could not be drawn. The quality of evidence was graded on level 3 .

\section{- Balance of benefits and harms}

Difficulties with swallowing, with chewing and choking are the most frequently described types of feeding difficulties necessitating different nutritional strategies depending on the severity of the feeding difficulties. Because most patients are diagnosed with feeding difficulties before the age of one and this is the period of rapid growth and development, the benefits of appropriate and early nutritional intervention outweigh the possible harms.

\section{- Outcome importance}

With regard to the optimal growth and development of young children it is of the utmost importance to focus on nutritional therapy, growth and follow-up. 


\section{- Costs and resources}

The impact on costs and resources of the given recommendations will vary per member state, depending on the available care providers and facilities. The recommendations concern the essential requirements for adequate treatment of patients with CFM and should thus be implemented. Costs are lowest and resources are most efficiently used when care for craniofacial disorders is centralised in a limited number of expert centres per member state. A general rule that can be applied is one expert centre per 10 million inhabitants.

\section{- Inequity of the recommendation}

The goal of the European Reference Networks (ERNs) is to eliminate inequality within Europe with regard to care for patients with rare diseases. At present, not every member state offers an expert centre for CFM, or the level of provided care does not (yet) meet all the requirements that are outlined in this guideline. By defining the baseline of required care for CFM, this guideline will help these member states to reach the appropriate level. The ERN on craniofacial anomalies and ENT disorders (ERN-CRANIO) can guide a patient in Europe to the available centres of expertise (www.ern-cranio.eu) and can support care providers with diagnosis and treatment advice.

\section{- Feasibility of the recommendation}

Recommendations refer to the general requirements for delivering optimal healthcare and are discussed with members from participating European countries. Quality of care was paramount in the discussions. Centralisation is proposed as one of the core values. However, in some countries the national organisation of healthcare might impede centralisation. National implementation of the ERNs that fits the situation of each country is necessary. For the member states with the lowest number of inhabitants, the establishment of a craniofacial centre might not be feasible, and collaboration with a craniofacial centre in the surrounding countries can be an option.

\section{- Acceptability of the recommendation}

It is expected that all stakeholders want to apply and will be applying the recommendations, because they are employed in ERNacknowledged institutions. National implementation plans are necessary to ensure that recommendations fit the situation in each country. In addition, not all countries participating in the ERNCRANIO are represented in the guideline development group, and new members will join within the coming years. For these countries, acceptance and implementation of ERN guidelines such as this guideline on CFM is obligatory.

\section{Rationale of the recommendations}

The role of a multidisciplinary team to guide nutritional therapy has been advocated by many societies to improve nutritional intake and to optimise growth. In case of children with CFM a paediatrician or a paediatric gastroenterologist, speech and language therapist and dietician are involved in a multidisciplinary team. When children need tube feeding, a speech and language therapist has to be involved in follow-up of oral-motor behaviour. The indication for a gastrostomy has to be discussed in close cooperation with a paediatric gastro-enterologist. After discharge from the hospital monitoring of appropriate growth is needed and it should be clear who is responsible for growth and development in follow-up.

Recommendations

- Children with craniofacial microsomia with feeding difficulties should be treated by a multidisciplinary team.
- Feeding strategies are guided by the severity of feeding difficulties.

\section{Research gap}

The literature on treatment modalities for feeding difficulties in CFM is retrospective and no comparative studies have been done. Future prospective studies on this subject, with long-term follow-up and patient specific outcomes, are needed. The ICHOM Craniofacial Microsomia standard set will help to create a good prospective dataset from a large number of patients with CFM (9).

\section{References}

1. Cousley RR, Calvert ML. Current concepts in the understanding and management of hemifacial microsomia. Br J Plast Surg. 1997;50(7):536-51.

2. Adam MP AH, Pagon RA, et al., editors. GeneReviews [Internet]. Seattle (WA): University of Washington, Seattle; 1993-2019.

3. Caron CJ, Pluijmers BI, Joosten KF, Mathijssen IM, van der Schroeff MP, Dunaway DJ, et al. Feeding difficulties in craniofacial microsomia: a systematic review. Int $\mathrm{J}$ Oral Maxillofac Surg. 2015;44(6):732-7.

4. Cohen MS, Samango-Sprouse CA, Stern HJ, Custer DA, Vaught DR, Saal HM, et al. Neurodevelopmental profile of infants and toddlers with oculo-auriculo-vertebral spectrum and the correlation of prognosis with physical findings. Am J Med Genet. 1995;60(6):535-40.

5. Stromland K, Miller M, Sjogreen L, Johansson M, Joelsson BM, Billstedt E, et al. Oculo-auriculo-vertebral spectrum: associated anomalies, functional deficits and possible developmental risk factors. Am J Med Genet A. 2007;143A(12):131725.

6. Caron C, Pluijmers BI, Joosten KFM, Dunaway D, Padwa BL, Wolvius EB, et al. Feeding difficulties in craniofacial microsomia: A multicenter retrospective analysis of 755 patients. J Craniomaxillofac Surg. 2018;46(10):1777-82.

7. van de Lande LS, Caron C, Pluijmers BI, Joosten KFM, Streppel M, Dunaway DJ, et al. Evaluation of Swallow Function in Patients with Craniofacial Microsomia: A Retrospective Study. Dysphagia. 2018;33(2):234-42.

8. Brotto D, Manara R, Vio S, Ghiselli S, Cantone E, Mardari R, et al. Salivary glands abnormalities in oculo-auriculo-vertebral spectrum. Clin Oral Investig. 2018;22(1):395-400.

9. International Consortium for Health Outcomes Measurement. ICHOM Craniofacial Microsomia Data Collection Reference Guide. 2017.

\section{CHAPTER 4. SCREENING, MONITORING AND INDICATION FOR TREATMENT}

\subsection{Speech and Language difficulties in craniofacial microsomia \\ Introduction}

Speech, language and communication are crucial to the development of children and young people which impact on educational achievement; emotional, social and mental wellbeing, and opportunities in life. Individuals with speech, language and communication needs (SLCN) present with a range of receptive and expressive difficulties. These include: verbal, written or non-verbal expression; understanding spoken or written word, body language and facial expressions; listening and remembering; expressing feelings and 
emotions in an appropriate and adaptive way; or being able to relate to others in socially acceptable ways (1).

Oral-facial features of CFM such as mandibular hypoplasia, tongue anomalies, facial asymmetry, facial weakness, teeth anomalies, dental malocclusion, cleft palate and macrosomia may all contribute to speech difficulties, in particular articulation difficulties $(2,3)$. Presentation may range from mild difficulties affecting the appearance of speech but not intelligibility to severe articulatory errors making speech unintelligible. Cleft lip/palate is reported in $15-22 \%$ of the patients with CFM (3-5). This may result in velopharyngeal dysfunction (VPD) and atypical articulatory patterns knowns as cleft speech characteristics (CSCs), both of which can compromise speech intelligibility. VPD is characterised by hypernasal resonance and the nasal airflow features of nasal turbulence and emission, which may warrant surgical intervention if intelligibility is compromised. In the absence of a cleft palate, there remains a risk of VPD due to asymmetry and hypoplasticity on the microsomic side which can affect the velopharyngeal anatomy and function (6). VPD has also been reported to occur with palatal paresis or paralysis (7).

Good hearing is essential for speech and language development. Patients with CFM are at an increased risk of hearing problems due to external and/or internal ear abnormalities, auditory canal atresia, and eustachian tube dysfunction (8). Conclusions of literature and recommendations for care of hearing difficulties in CFM is described in chapter 4.4. The condition of CFM can predispose children to early language delay which may be unrelated to hearing impairment. Language difficulties in older children have been reported.

To gain a deeper understanding of the broad communication profile in CFM and to inform recommendations on screening, assessment and management, the following guiding questions are posed:

4.3.1 What is the type, prevalence and severity of speech and language difficulties in craniofacial microsomia?

4.3.2 What is the policy for screening and monitoring of speech and language difficulties in patients with craniofacial microsomia?

4.3.3 What are the indications and policy for treatment of speech and language difficulties in patients with craniofacial microsomia?

Early detection of delays in the development of speech and language is imperative to facilitate good communication, social interaction and improved quality of life $(9,10)$. As patients with CFM may be of increased risk for and language difficulties, early screening is indicated.

Literature search

A systematic search of the evidence base was performed to identify all available literature on craniofacial microsomia and appropriate synonyms. The search was conducted in Embase, Pubmed/Medline Ovid. The full search strategy is reported in the supplementary material.

Inclusion and exclusion criteria:

$\begin{array}{ll}\text { Type of studies } & \text { - Original articles } \\ & \text { - Systematic review of sufficient quality: } \\ & \text { - The question in the systematic review matches the question of } \\ \text { the guideline. } & \\ \text { - The search of the systematic review was conducted in at least } & \text { two relevant databases, such as the Cochrane Library, } \\ & \text { Medline/Pubmed. } \\ \text { - The full search strategy was reported. } \\ \text { - No relevant items were missing in the search strategy. } \\ \text { Type of patients } \quad \text { - Patients with craniofacial microsomia }\end{array}$

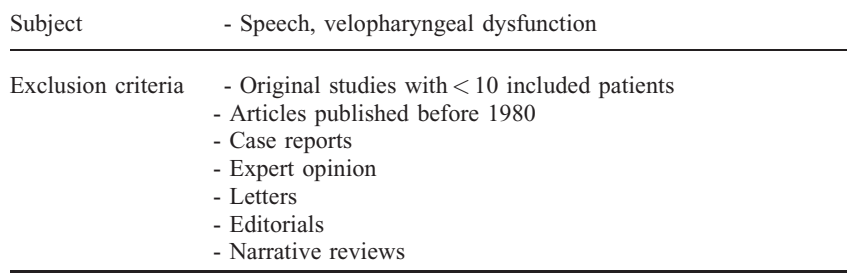

Literature was screened on title and abstract. Five articles were included: a cross-sectional study by Funayama et al. (11), a retrospective cohort study by Strömland et al. (12), a cross-sectional study by D'Antonio et al. (13), a cross-sectional study by Cohen et al. (14), and a retrospective cohort study by Chen et al. (15).

4.3.1 What is the type, prevalence and severity of speech difficulties in craniofacial microsomia?

The literature was specifically examined for evidence on the type of speech and language difficulties, including resonance disorders, articulation difficulties voice disorders, the prevalence and severity (rating scales) in CFM (Appendix 1, http://links.lww.$\mathrm{com} / \mathrm{SCS} / \mathrm{B} 697)$. Since this question does not relate to interventions or diagnostics, no considerations, rationale or recommendations are provided.

\section{REVIEW OF LITERATURE}

A range of communication difficulties and their associated prevalence in CFM was reported inconsistently across the included studies, specifically language difficulties, velopharyngeal dysfunction, articulation difficulties secondary to tongue abnormalities, facial nerve palsy, social communication difficulties and voice disorder. Oral motor difficulties were referred to in one study, while a further paper reported on tracheostomy management for airway difficulties, with no reference to the impact on communication. Many of these communication features were not systematically reported using validated assessment tools, with inadequate descriptive reporting as well as the use of subjective terms and ratings. Furthermore, the description of the individual communication difficulties was inaccurately described in a number of papers; e.g. non-verbal children described as having speech articulation difficulties, when in fact, this is a language deficit. 'Speech difficulties' is not defined in any study, making it unclear if the reported speech difficulties are structural, developmental or disordered. This is important in terms of the nature of the communication difficulty reported and the intervention that is recommended.

\section{Receptive and expressive language skills}

Cohen et al. studied communication disorders in patients with CFM (14). In this study, receptive- and expressive language skills were found to be -2 standard deviations below the normal population in one-third of the cohort $(n=24)$. Receptive and expressive language was assessed using the Early Language Milestone Scales for children aged one month to three. This is a norm-referenced language screen that uses parental report, clinical observation and direct testing. The Expressive One-Word Picture Vocabulary Test or Peabody Picture Vocabulary Test was used with older children aged three to five years to assess expressive and receptive vocabulary respectively.

The same study also found that the children with oral-facial hypotonia, diagnosed with neurological examination, had a significantly lower expressive language score with an average of 69 versus 100 in the "normal tone" group. (14). Oral-facial hypotonia was diagnosed following a neurological examination, which was not further specified. In addition, receptive and expressive language 
scores scores were $2 \mathrm{~s}$.d. below the mean of the normal population in $32 \%(n=6)$ and $37 \%(n=7)$ of the patients respectively. Patients with unilateral CFM scored significantly higher on the receptive and expressive language scores compared to bilateral patients (respectively 100 versus $58, \mathrm{p}=0.003 ; 100$ versus $64, \mathrm{p}=0.02$ ) (14).

\section{Speech articulation difficulties}

Most facial structures are underdeveloped in patients with CFM, including tongue anomalies which can contribute to atypical speech articulation (15). Chen and colleagues' (15) retrospective case-note review of 167 patients revealed that only eight patients $(4.8 \%)$ had a tongue anomaly, e.g. ankyloglossia, tongue hypoplasia or a bifid tongue. Seven of these 8 cases had speech delay or deficits, though the nature or severity of the articulation difficulties is not described. Resolution of these speech patterns occurred in seven cases, five of whom had speech therapy, while the eighth patient was non-verbal. Alternative communication modes for this patient were not reported. The study also included a prospective arm, in which 55 patients with CFM underwent evaluation for tongue anomalies. Patients with a higher Mandible or Soft tissue score on the O.M.E.N.S. scale were found to be at increased risk of tongue anomalies: a correlation coefficient of $0.527, \mathrm{p}=<0.001$ was found for the Mandible score while a correlation coefficient $0.464, p=<0.001$ was confirmed for the Soft Tissue score. The presence of tongue anomalies was much higher in this prospective sample, yielding a $44 \%$ prevalence. The type of speech evaluation is not described. However, speech difficulties were reported in 21 patients (38\%) while four children were described as being deaf and non-verbal. As nineteen patients were too young to be evaluated, the incidence of speech difficulties in this cohort may indeed be higher. It is unclear what proportion of children with a tongue deformity had a speech deficit.

Strömland et al reported on oral-motor function and speech characteristics using the Mun-H-Center Observation chart (12). This observation chart is described as a record chart to collect clinical observations made not only about speech and oral motor function, but also orofacial morphology and odontology. This is not a validated assessment tool nor is reference made to the use of a formal speech assessment with a standardised speech sample, making it difficult to interpret speech findings and to appreciate the impact upon intelligibility. Of the 15 patients aged between $2-$ 17 years old, $53 \%$ were found to have articulation difficulties. A general description of the severity of the speech difficulty is provided, i.e. five children were non-verbal, one child had 'severe' speech difficulties while two children had speech articulation difficulties considered to be 'slight'. No information is provided regarding the articulatory patterns or phonetic deviations is provided, underscoring the limited detail of the nature of the speech sound disorder. The study alludes to a potential association between these speech and language difficulties and the children's cognitive function. Six children were reported to present with drooling, with no further information provided.

\section{Velopharyngeal dysfunction}

D'Antonio and colleagues (13) conducted a cross-sectional study by means of a retrospective chart review and a prospective examination of a portion of the same patients to examine the pharyngeal and laryngeal structure and function of patients with CFM. Cleft palate was a reported feature in $15 \%$ of the sample of 41 patients, while $10 \%$ had a submucous cleft palate. Hypernasality was noted in $39 \%$ of the cohort with less than half of these having a cleft palate ( 9 patients without a palatal cleft and 6 patients with a cleft palate). Of cases without a cleft palate, $29 \%$ presented with hypernasal resonance or nasal emission. Two patients with cleft palate had oral resonance.

In the prospective arm of the study (13), perceptual evaluation of velopharyngeal function for speech of 19 patients demonstrated hypernasality in five patients $(26 \%)$ and hyponasality in three patients $(16 \%)$. Of the five patients with hypernasality, two had a cleft palate. Twenty-three patients in this study arm underwent nasendoscopic evaluation of the velopharyngeal mechanism during speech, though four of these were later excluded due to the inability to interpret the speech findings as they were too young or non-verbal at the time of investigation. Nasendoscopic view revealed asymmetric velopharyngeal function in $35 \%$ of this cohort. $13 \%$ presented with incomplete velopharyngeal closure during speech, with anteroposterior narrowing of the velopharynx reported in $9 \%$ of the sample. Aerodynamic evaluations were also completed for 18 of the 23 cases, adding to the rigorous investigative protocol. These aerodynamic evaluations supported the perceptual findings in all cases but one, which was felt to have been influenced by the patient's variable performance between tests. The study concludes that there may be a relationship VPD and the severity of the mandibular deformity in this condition, given that the entire cohort with stage III mandibular deviation presented with asymmetrical velopharyngeal function. By contrast, less than a fifth of the group with stage I mandibular dysmorphology had an asymmetrical velopharynx.

Articulation, resonance and intelligibility were reported using a 7-point scale, however there is no reported validation of this, nor description of the scale (13). Impaired articulation was reported in $74 \%$ of the sample, with five cases in the severe range, four considered to have moderate difficulty and five having mild difficulty. However, no transcription of the phonetic or phonological deviations are provided. Intelligibility was reported to be impaired in $56 \%$ of cases, with $5 / 23$ cases rated as severely unintelligible.

Funayama et al. studied 52 patients with CFM and reported on the correlation between VPD and the velopharyngeal phenotypes (11). 48 patients with unilateral CFM and isolated microtia were included, with an additional 4 patients with a repaired cleft palate in CFM evaluated for comparison. 50\% $(n=24)$ of the patients without a cleft lip/palate had a unilateral hypodynamic palate, though interestingly, only seven of these 24 patients (29\%) had VPD. Velopharyngeal dysfunction was found to correlate with mandibular deficiency (Spearman's rank correlation coefficient: $1.00, \mathrm{p}=<0.01$ ) and Soft Tissue score (Spearman's rank correlation coefficient: $0.74, p=<0.05$ ) on the O.M.E.N.S. classification (orbit, mandible, ear, nerve, soft tissue) (11). Correlations between VPD and macrostomia ( 3 out of 7 patients had VPD, $\mathrm{p}=<0.05$ ) and cognitive function ( 5 of the 5 patients had VPD, $\mathrm{p}=<0.01$ ) were analysed. Of the 4 patients with cleft lip/palate, all presented with VPD. Their post mandibular distraction nasendoscopic investigations revealed decreased unilateral velopharyngeal movement on the microsomic side. Two of these patients, both of whom had UCLP, underwent secondary speech surgery to manage their hypernasality.

\section{Voice}

D'Antonio and colleagues (13) reported hoarseness in 24\% of the retrospective cohort. This study examined laryngeal function under endoscopy in 22 patients in the prospective study arm. A range of observations were reported, occurring in $86 \%$ of cases, including infantile larynges, anteroposterior narrowing, asymmetrical arytenoidal movement, significant asymmetrical laryngeal movement. Vocal cord and epiglottic abnormalities were also reported (13). The authors did not report a relationship between 
these laryngeal pathologies and mandibular hypoplasia, though no statistical testing is reported.

\section{Impact of hearing difficulties on communication development}

Chapter 4.4 Hearing focuses on hearing loss in CFM. However, two articles described that hearing loss may have an effect on speech sound and language development in patients with CFM. Hearing loss was observed in 22 of the 24 children with CFM in the Neurodevelopmental Profile Study by Cohen et al. (14). They hypothesized that this may have contributed to the low language scores. This however was not analysed due to the small size of the cohort. In another study by Strömland et al. (12), 15 of the 18 patients were documented as having a hearing loss, bilateral hearing loss being most prevalent $(\mathrm{N}=11)$. Seven patients with bilateral hearing loss presented with speech difficulties. Information on hearing intervention i.e. hearing aid use or surgical intervention was not included. No statistical tests were performed to assess the relationship between speech difficulties and hearing defects. In this study, speech difficulties may not have arisen exclusively due to hearing loss, but were likely to have been influenced by the comorbid factors of cognitive impairment and oral facial malformation.

The authors of both studies recommend screening for hearing problems early in childhood and repeat hearing tests if the results are inconclusive $(12,14)$.

\section{Social communication difficulties}

Strömland et al. reported severe social communication difficulties and limited communication, rendering a diagnosis of autistic spectrum disorder in $11 \%$ of children (12).

\section{Conclusions}

\section{Prevalence \& Type}

Communication difficulties that may present in craniofacial microsomia include: velopharyngeal dysfunction; dysphonia; impaired speech articulation; receptive and expressive language difficulties and social communication difficulties

Prevalence rates of speech difficulties reported in CFM vary from $38 \%-74 \%$.

Receptive and expressive language difficulties are reported in $33 \%$ of the reported cohort of 24 patients.

Dysphonia was reported in $24 \%$ of a retrospective cohort of 41 patients.

Social Communication difficulties including autistic spectrum disorder, were reported in $11 \%$ of the cohort.

$\operatorname{Ref}(11-15)$

\section{Prevalence}

The reported prevalence of velopharyngeal dysfunction in CFM without cleft palate is $15 \%$. In a sample of 48 patients, submucous cleft palate was not reported.

Velopharyngeal dysfunction was observed in $35 \%$ of a cohort due to asymmetric velopharyngeal closure. No comparison with a healthy control group was made.

$\operatorname{Ref}(11,13)$
4.3.2 What is the policy for screening and monitoring of speech and language difficulties in patients with craniofacial microsomia?

\section{Review of literature}

The literature was specifically examined for evidence on the consequences and impact of screening and monitoring, the available screening tests and procedures for monitoring, and responsibilities for screening and monitoring (appendix 1, http://links.lww.com/ SCS/B697). Unfortunately, no literature is available on the policy for screening and/or monitoring of speech and language difficulties in patients with CFM. Considerations for this question were based on the evidence and expert opinion. Therefore no conclusions were written. Considerations for this question were only based on expert opinion.

\section{Considerations}

\section{- Overall quality of evidence}

The evidence currently available on speech difficulties in CFM is limited. Only five studies met the inclusion criteria, all of which profiled different speech and language characteristics. Three studies had prospective designs $(11,12,14)$, a further study had both a retrospective chart review and a prospective arm (15), while the remaining study was a retrospective cross-sectional chart review. A range of methodological weaknesses were found across the studies, ranging from a lack of detail about consecutiveness and comparison groups, to ill-defined and incorrect use of terminology relating to the communication aspects being profiled. Furthermore, all but one study did not report the use of a validated assessment tool. These weaknesses are reflective of the inherent potential for bias within the studies. Thus, these studies represent Level III evidence.

\section{- Balance of benefits and harms}

Children with CFM warrant early screening of their preverbal and early language skills due to the risk of visual and hearing impairment as well as oral-facial difference that can impact on early social development and receptive and expressive language skills. Children with the condition are at increased risk of receptive and expressive language difficulties. Conti-Ramsden and colleagues (16) found that when these language difficulties persist into adolescence and adulthood in the non-CFM population, lower academic and vocational qualifications than their peers were found. Therefore, early screening and intervention for language difficulties is advised. Articulation difficulties, velopharyngeal dysfunction and voice disorders and can all result in reduced intelligibility which impacts on self-esteem and compromises an individual's QoL.

\section{- Outcome importance}

To date, patient reported outcomes for speech and language in CFM have not been systematically reported in the literature. However, expert opinion indicates that patients attending CFM clinics report positive improvements in interactions with family, peers, and the wider community following speech and language intervention. Increased communication confidence is reported, demonstrating the key strands of the ICF (International Classification of Functioning) of participation, functioning, impairment and wellbeing (17).

\section{- Costs and resources}

The impact on costs and resources of the given recommendations will vary per member state, depending on the available care 
providers and facilities. The recommendations concern the essential requirements for adequate treatment of patients with CFM and should thus be implemented. Costs are lowest and resources are most efficiently used when care for craniofacial disorders is centralised in a limited number of expert centres per member state. A general rule that can be applied is one expert centre per 10 million inhabitants.

\section{- Inequity of the recommendation}

The goal of the European Reference Networks (ERNs) is to eliminate inequality within Europe with regard to care for patients with rare diseases. At present, not every member state offers an expert centre for CFM, or the level of provided care does not (yet) meet all the requirements that are outlined in this guideline. By defining the baseline of required care for CFM, this guideline will help these member states to reach the appropriate level. The ERN on craniofacial anomalies and ENT disorders (ERN-CRANIO) can guide a patient in Europe to the available centres of expertise (www.ern-cranio.eu) and can support care providers with diagnosis and treatment advice.

\section{- Feasibility of the recommendation}

Recommendations refer to the general requirements for delivering optimal healthcare and are discussed with members from participating European countries. Quality of care was paramount in the discussions. Centralisation is proposed as one of the core values. However, in some countries the national organisation of healthcare might impede centralisation. National implementation of the ERNs that fits the situation of each country is necessary. For the member states with the lowest number of inhabitants, the establishment of a craniofacial centre might not be feasible, and collaboration with a craniofacial centre in the surrounding countries can be an option.

\section{- Acceptability of the recommendation}

It is expected that all stakeholders want to apply and will be applying the recommendations, because they are employed in ERNacknowledged institutions. National implementation plans are necessary to ensure that recommendations fit the situation in each country. In addition, not all countries participating in the ERNCRANIO are represented in the guideline development group, and new members will join within the coming years. For these countries, acceptance and implementation of ERN guidelines such as this guideline on CFM is obligatory.

\section{Rationale of the recommendations}

Individuals with CFM are at risk of communication difficulties due to oral-facial anomalies, cleft palate and velopharyngeal dysfunction, and/or hearing impairment. Other features that can adversely impact communication skills in CFM are: facial nerve palsy which can affect facial expression (18), velopharyngeal function (7); airway difficulties requiring tracheostomy intervention and its impact on speech articulation, language development and voice; cognitive impairment ranging from neurotypical to severely impaired with associated specific language difficulties $(12,14)$ some of the time; and visual impairment as it may affect play, motor, cognitive, social and communication skills in children $(12,19)$.

Individuals with CFM are at risk of communication difficulties due to the following inter-related predisposing factors which are shown in the diagram below:

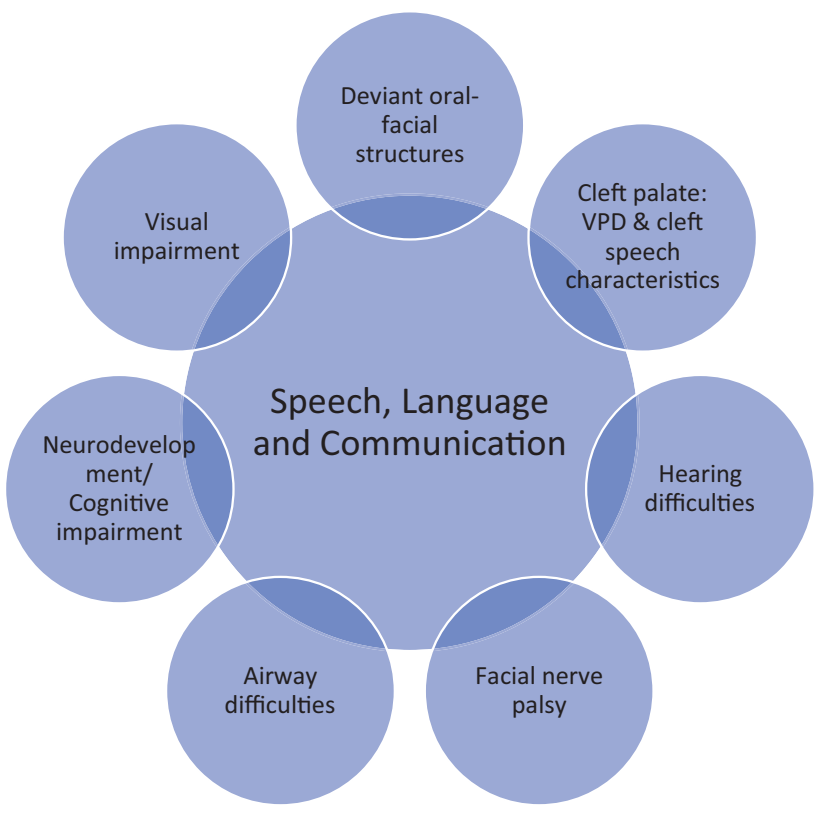

At the age of two years, the child's phonetic repertoire should be initially assessed to examine his/her velopharyngeal function for speech due to the potential risk of VPD related to a unilateral hypodynamic palate and/or cleft palate. If there are concerns about velopharyngeal dysfunction (VPD), a referral to the specialist Velopharyngeal Investigations clinic attached to a cleft centre is recommended from age three onwards. If there are concerns regarding cleft speech characteristics, therapy is recommended. The specialist Cleft-Craniofacial SLT may deliver this or support the community SLT in providing this intervention. After five years, bi-annual screening of velopharyngeal function for speech is recommended until age 20 . Speech screening should follow the guidelines set out in the Universal Parameters for Reporting Speech Outcomes in Individuals with Cleft Palate (20). This consists of obtaining a spontaneous speech sample, rote speech elicitation (e.g. counting, nursery rhyme), single words for younger children and sentence repetition for all speech sounds to examine the phonetic repertoire and the features of resonance and nasal airflow (Table 1 in Appendix 5, http://links.lww.com/SCS/B697). The parameters of resonance and nasal airflow can be rated according to severity (Table 1 in Appendix 5, http://links.lww.com/SCS/B697).

In the case of non-cleft CFM, speech requires ongoing monitoring throughout childhood as the child's speech and language skills mature, to ensure that any emerging velopharyngeal dysfunction is detected. In later childhood, as the nasopharyngeal tract alters with maturity and with adenoidal involution, previously absent features of VPD, including hypernasality and nasal airflow errors, may emerge. It is thus important that the Speech and Language Therapist acknowledges the changing profile of resonance and speech over time and does not discharge the patient in early childhood. Examination of oral-facial structure and function is strongly recommended when evaluating speech. Features of CFM such as tongue anomalies, orofacial hypotonia, macrostomia, dental malocclusion, mandibular hypoplasia contribute to articulation difficulties. Individuals with tracheostomy should be screened for speaking valve suitability or an augmentative and alternative communication system (communication methods used to supplement or replace speech or writing). Given the reported social communication difficulties that may present in this population, 
either occurring in isolation or related to visual impairment, early screening and monitoring of social interaction skills are recommended from the age of 9 months onwards. It is important to acknowledge that social communication difficulties may become more apparent in later childhood as abstract language features more in social interactions and the educational curriculum.

\section{Recommendations}

- Screen preverbal communication and babbling skills at the age of nine months to decide if intervention is warranted.

- Evaluate receptive and expressive language skills at the age of two years and biannually until the age of eight years in all patients with craniofacial microsomia. Those identified with difficulties should be referred to their community speech and language therapist service for ongoing intervention.

- Oral-facial evaluation of structure and function is recommended at each screening consultation to examine any impact of asymmetry on speech production. This should include examination of facial symmetry, lips, dental occlusion and intra-oral examination of tongue movement, dentition, hard palate and soft palate movement on sustained 'ah' vowel.

- Screen patients with tracheostomy for speaking valve suitability or an augmentative and alternative communication system.

- Social communication skills should be monitored in tangent with all of the afore-mentioned communication skills.

- Children with craniofacial microsomia and associated cleft palate should be screened annually from $2-5$ years by the Cleft-Craniofacial speech and language therapist and should follow the local Cleft Palate Protocol. Velopharyngeal dysfunction should be assessed from the age of two years or when verbal output has emerged.

- Children with craniofacial microsomia without a cleft palate should also be screened at the age of two years to examine for potential risk of velopharyngeal dysfunction related to their asymmetrical structure. If velopharyngeal dysfunction is identified, these children should follow the same pathway as children with a cleft palate.

\section{Research gap}

No literature is available regarding the policy of screening and monitoring of speech difficulties in patients with craniofacial microsomia. Availability of studies could help to develop a better screening and monitoring.

4.3.3 What are the indications and policy for treatment of speech difficulties in patients with craniofacial microsomia?

The literature was specifically examined for evidence on treatment options, symptoms, requirements, complications, and advantages and disadvantages of treatment of speech problems (appendix 1, http://links.lww.com/SCS/B697). Unfortunately, no literature is available on the indications and policy for treatment of speech and language difficulties in patients with CFM. However, there is evidence for the management of VPD in the cleft palate only population which can be applied to CFM. In addition, the local policies and guidelines that have been developed in different countries (21) for the management of receptive and expressive language difficulties can also be used with individuals CFM. Considerations for these questions were based on expert opinion and on the wider literature on management of speech and language difficulties.

\section{Considerations \\ - Overall quality of evidence}

Because there is no literature available the recommendations are based on expert opinion with a low quality of evidence.

\section{- Balance of benefits and harms}

Early treatment of speech and language difficulties is crucial to minimise lower school achievement and psychosocial sequelae (22). Early intervention can facilitate improved parent awareness of their child's communication difficulty, enabling them to support their child appropriately in their everyday interactions.

\section{- Outcome importance}

Early intervention and ongoing therapy for language, articulation and social communication difficulties contributes to enhanced patient QoL. Likewise, improving the child's VP function for speech, can contribute to this. The community SLT working with a child with CFM may not be aware of the management of VP function; therefore, regular review by a specialist craniofacial/cleft SLT will ensure good clinical practice.

\section{- Costs and resources}

The impact on costs and resources of the given recommendations will vary per member state, depending on the available care providers and facilities. The recommendations concern the essential requirements for adequate treatment of patients with CFM and should thus be implemented. Costs are lowest and resources are most efficiently used when care for craniofacial disorders is centralised in a limited number of expert centres per member state. A general rule that can be applied is one expert centre per 10 million inhabitants.

\section{- Inequity of the recommendation}

The goal of the European Reference Networks (ERNs) is to eliminate inequality within Europe with regard to care for patients with rare diseases. At present, not every member state offers an expert centre for CFM, or the level of provided care does not (yet) meet all the requirements that are outlined in this guideline. By defining the baseline of required care for CFM, this guideline will help these member states to reach the appropriate level. The ERN on craniofacial anomalies and ENT disorders (ERN-CRANIO) can guide a patient in Europe to the available centres of expertise (www.ern-cranio.eu) and can support care providers with diagnosis and treatment advice.

\section{- Feasibility of the recommendation}

Recommendations refer to the general requirements for delivering optimal healthcare and are discussed with members from participating European countries. Quality of care was paramount in the discussions. Centralisation is proposed as one of the core values. However, in some countries the national organisation of healthcare might impede centralisation. National implementation of the ERNs that fits the situation of each country is necessary. For the member states with the lowest number of inhabitants, the establishment of a craniofacial centre might not be feasible, and collaboration with a craniofacial centre in the surrounding countries can be an option. 


\section{- Acceptability of the recommendation}

It is expected that all stakeholders want to apply and will be applying the recommendations, because they are employed in ERNacknowledged institutions. National implementation plans are necessary to ensure that recommendations fit the situation in each country. In addition, not all countries participating in the ERNCRANIO are represented in the guideline development group, and new members will join within the coming years. For these countries, acceptance and implementation of ERN guidelines such as this guideline on CFM is obligatory.

\section{Rationale of the recommendations}

Individuals with CFM can present with a range of communication difficulties, not least, early language delay, articulation disorders and velopharyngeal dysfunction. These may be related to one or a combination of the following: cleft palate, asymmetrical oralfacial structures, hearing difficulties and visual impairment. In addition, this population can also present with difficulties in receptive and expressive language skills, requiring alternative communication modes such as signing in early childhood. Where the screening/evaluation has highlighted difficulties, any of the following recommendations may apply and should be considered on an individual basis. The duration and intensity of treatment is not stipulated, as this will depend on the needs of the child, engagement of the child and family in the intervention process, available resources and local policies for speech and language therapy services. These recommendations have been made to facilitate the best long-term outcome for the individual and to decrease the burden of care for all agencies involved, as the literature shows that poor outcomes for literacy, employability and mental health in adults (23).

\section{Recommendations}

- Recommend early language stimulation for delayed babble onset from nine months.

- Facilitate receptive and expressive language development using a range of behavioural techniques such as modelling, imitation, repetition and extension.

- Patients with cleft speech characteristics should have articulation therapy when identified. Direct therapy using an articulation approach is recommended from age three onwards

- Monitor patients with tracheostomy and speaking valve use on a regular base.

- Introduce low or high tech augmentative and alternative communication systems to children who are non-verbal or whose speech is unintelligible. These include gestures, signing, symbols, word boards, communication boards and books, as well as Voice Output Communication Aids (VOCAs). A low-tech system such as signing can be introduced from one.

- Intervention for social communication difficulties is recommended; e.g. development of non-verbal communication skills (e.g. eye contact, turn-taking); conversational skills, recognitions of emotions and emotional regulation.

\section{Research gap}

No literature is available regarding indications and policy for treatment of speech difficulties in patients with craniofacial microsomia. Availability of studies could help determine an optimal treatment strategy.

\section{References}

1. RCSLT. Resource Manual For Commissioning And Planning Services For SLCN: Speech and Language Impairment. London: RCSLT. 2009.

2. Fan WS, Mulliken JB, Padwa BL. An association between hemifacial microsomia and facial clefting. J Oral Maxillofac Surg. 2005;63(3):330-4.

3. Caron C, Pluijmers BI, Wolvius EB, Looman CWN, Bulstrode $\mathrm{N}$, Evans RD, et al. Craniofacial and extracraniofacial anomalies in craniofacial microsomia: a multicenter study of 755 patients'. J Craniomaxillofac Surg. 2017;45(8):130210.

4. Cousley RR, Calvert ML. Current concepts in the understanding and management of hemifacial microsomia. Br J Plast Surg. 1997;50(7):536-51.

5. Adam MP AH, Pagon RA, et al., editors. GeneReviews [Internet]. Seattle (WA): University of Washington, Seattle; 1993-2019.

6. Shprintzen RJ, Croft CB, Berkman MD, Rakoff SJ. Velopharyngeal insufficiency in the facio-auriculo-vertebral malformation complex. Cleft Palate J. 1980;17(2):132-7.

7. Luce EA, McGibbon B, Hoopes JE. Velopharyngeal insufficiency in hemifacial microsomia. Plast Reconstr Surg. 1977;60(4):602-6.

8. Mitchell RM, Saltzman BS, Norton SJ, Harrison RG, Heike CL, Luquetti DV, et al. Hearing Loss in Children With Craniofacial Microsomia. Cleft Palate Craniofac J. 2017;54 (6):656-63.

9. Birgfeld CB, Heike C. Craniofacial microsomia. Semin Plast Surg. 2012;26(2):91-104.

10. Roberts RM, Shute R. Children's experience of living with a craniofacial condition: perspectives of children and parents. Clin Child Psychol Psychiatry. 2011;16(3):317-34.

11. Funayama E, Igawa HH, Nishizawa N, Oyama A, Yamamoto Y. Velopharyngeal insufficiency in hemifacial microsomia: analysis of correlated factors. Otolaryngol Head Neck Surg. 2007;136(1):33-7.

12. Stromland K, Miller M, Sjogreen L, Johansson M, Joelsson BM, Billstedt E, et al. Oculo-auriculo-vertebral spectrum: associated anomalies, functional deficits and possible developmental risk factors. Am J Med Genet A. 2007;143A(12): 1317-25.

13. D'Antonio LL, Rice RD, Jr., Fink SC. Evaluation of pharyngeal and laryngeal structure and function in patients with oculo-auriculo-vertebral spectrum. Cleft Palate Craniofac J. 1998;35(4):333-41.

14. Cohen MS, Samango-Sprouse CA, Stern HJ, Custer DA, Vaught DR, Saal HM, et al. Neurodevelopmental profile of infants and toddlers with oculo-auriculo-vertebral spectrum and the correlation of prognosis with physical findings. Am J Med Genet. 1995;60(6):535-40.

15. Chen EH, Reid RR, Chike-Obi C, Minugh-Purvis N, Whitaker LA, Puchala J, et al. Tongue dysmorphology in craniofacial microsomia. Plast Reconstr Surg. 2009;124(2):583-9.

16. Conti-Ramsden G, Durkin K, Toseeb U, Botting N, Pickles A. Education and employment outcomes of young adults with a history of developmental language disorder. Int J Lang Commun Disord. 2018;53(2):237-55.

17. WHO. Towards a Common Language for Functioning, Disability and Health ICF. 2002.

18. Hammal Z, Cohn JF, Wallace ER, Heike CL, Birgfeld CB, Oster H, et al. Facial Expressiveness in Infants With and Without Craniofacial Microsomia: Preliminary Findings. Cleft Palate Craniofac J. 2018;55(5):711-20. 
19. Chen D. Visual impairment in young children: A review of the literature with implications for working with families of diverse cultural and linguistic backgrounds. Early Childhood Research Institute on Culturally and Linguistically Appropriate Services, University of Illinois at Urbana-Champaign. 2001; Technical Report No 7:1-29.

20. Henningsson G, Kuehn DP, Sell D, Sweeney T, TrostCardamone JE, Whitehill TL, et al. Universal parameters for reporting speech outcomes in individuals with cleft palate. Cleft Palate Craniofac J. 2008;45(1):1-17.

21. Document IPPaG. Supporting Children with Developmental Language Disorder in Ireland. 2017.

22. Eadie P, Conway L, Hallenstein B, Mensah F, McKean C, Reilly S. Quality of life in children with developmental language disorder. Int J Lang Commun Disord. 2018;53(4): 799-810.

23. Law J, Rush R, Schoon I, Parsons S. Modeling developmental language difficulties from school entry into adulthood: literacy, mental health, and employment outcomes. J Speech Lang Hear Res. 2009;52(6):1401-16.

\section{CHAPTER 4. SCREENING, MONITORING AND INDICATION FOR TREATMENT}

\subsection{Hearing difficulties in craniofacial microsomia \\ Introduction}

Hearing is a sensory experience that facilitates communication and social interaction. Hearing impairment may lead to difficulties in learning, language and cognitive development, academic achievements, and can have a negative social impact on children (1). Hearing loss may be either conductive and/or sensorineural. Conductive hearing loss is based on any obstruction in the passage of sound through the outer ear, ear canal and/or middle ear. The inner ear, with the cochlea and vestibulocochlear nerve, processes the sound wave. Pathology in this system leads to sensorineural hearing loss. Mixed hearing loss is a combination of sensorineural and conductive hearing loss.

The degree of hearing loss is divided into mild, moderate, severe and profound hearing loss. Mild hearing loss is defined as a hearing loss of 26-40 decibels, moderate hearing loss as 41-60 decibels, severe hearing loss as 61-80 decibels, and profound hearing loss as over 81 decibels (2).

In the general population, the prevalence of hearing difficulties in childhood is approximately $0.08 \%$ (3). The incidence of sensorineural hearing loss is found to be $0.001 \%$ to $0.004 \%$, but in patients with craniofacial syndromes this increases to $3 \%$ to $4 \%$ (4).

Patients with craniofacial microsomia (CFM) may be at increased risk for reduced hearing due to various congenital anomalies, such as microtia, and outer and middle ear hypoplasia/ dysplasia. Also, patients with CFM are prone to have Eustachian tube dysfunction and recurrent ear infections, which increases the risk for hearing loss too (5). Besides conductive hearing loss, patients with CFM may be at increased risk for sensorineural hearing loss due to inner ear malformations.

Early detection and intervention of hearing loss is associated with improved outcomes in all children, hence the implementation of neonatal hearing screening programmes worldwide. To obtain more knowledge on hearing loss and impairment in CFM and to offer potential recommendations on (additional) screening and treatment, the following questions were posed:
4.4.1 What is the type, prevalence and severity of hearing difficulties in craniofacial microsomia?

4.4.2 What is the policy for screening and monitoring of hearing difficulties in patients with craniofacial microsomia?

4.4.3 What are the indications and policy for treatment of hearing difficulties in patients with craniofacial microsomia?

Literature search

A systematic search of literature was performed to identify all available literature on craniofacial microsomia and synonyms. The search was conducted in Embase, Pubmed/Medline Ovid. The full search strategy is reported in the supplementary material.

Inclusion and exclusion criteria:

Type of studies - Original articles

- Systematic review of sufficient quality:

- The question in the systematic review matches the question of the guideline.

- The search of the systematic review was conducted in at least two relevant databases, such as the Cochrane Library, Medline/Pubmed.

- The full search strategy was reported.

- No relevant items were missing in the search strategy.

\begin{tabular}{ll}
\hline Type of patients & - Patients with craniofacial microsomia \\
Subject & - Hearing, ear anomalies \\
Exclusion criteria & - Original studies with $<10$ included \\
& patients \\
& - Articles published before 1980 \\
& - Case reports \\
& - Expert opinion \\
& - Letters \\
& - Editorials \\
& - Narrative reviews \\
\hline
\end{tabular}

Literature was screened on title and abstract. A total of eighteen articles were included. Most were retrospective cohort studies: Bassila et al. (6), Carvalho et al. (4), Cousley et al. (7), Heike et al. (8), Hennersdorf et al. (9), Jacobsson et al. (10), Llano-Rivas et al. (11), Mitchell et al. (5), Rahbar et al. (12), Strömland et al. (13), and Wan et al. (14). Three studies were case series: Bisdas et al. (15), Engiz et al. (16), and Rosa et al. (17). Four crosssectional studies were included, by Cohen et al. (18), Davide and Renzo et al. (19), Goetze et al. (20), and Sleifer et al. (21). No articles on the indication or outcomes of treatment of hearing difficulties in patients with CFM were available.

4.4.1 What is the type, prevalence and severity of hearing difficulties in craniofacial microsomia?

The literature search for this question was directed towards more specific questions related to the type of hearing loss (conductive, sensorineural) and type of congenital malformations (outer, middle, or inner ear anomalies), prevalence and severity (mild, moderate, severe or profound hearing loss) of hearing difficulties in CFM (Appendix 1, http://links.lww.com/SCS/B697). Since this question does not relate to interventions or diagnostics, no considerations, rationale or recommendations are provided. 


\section{Review of literature}

The prevalence of unspecified, conductive, sensorineural and mixed hearing loss reported in literature is shown in Figure 1. The percentages in Figure 1 correspond to the prevalences reported in the included studies.

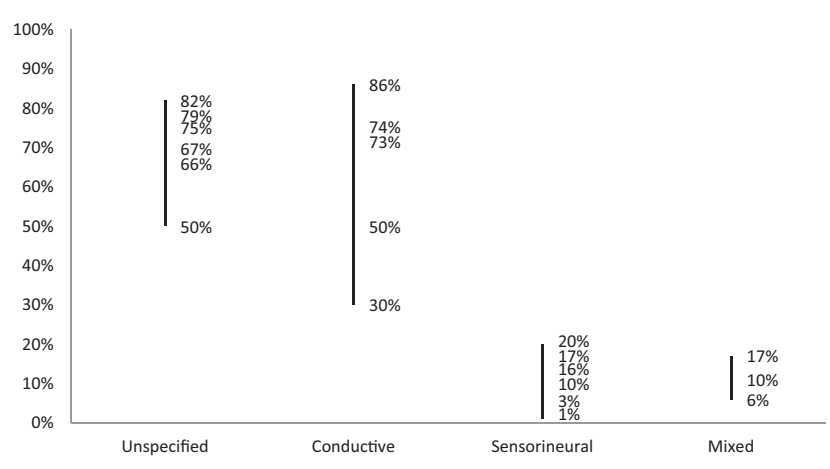

Figure 2. Prevalence of hearing loss in patients with craniofacial microsomia.

Mitchell et al. evaluated facial malformations and hearing loss in patients with CFM (5). A total of 79 patients with CFM were included: 39 patients had bilateral CFM and 40 patients had unilateral CFM. Microtia was present in $94 \%$ of the patients and hearing loss in $82 \%(n=65)$ of the patients. Of the patients with hearing loss, twelve patients had bilateral hearing loss and 53 patients had unilateral hearing loss. Interestingly, two patients with bilateral hearing loss had a normal ear phenotype and two patients had unilateral microtia. The severity of hearing loss was mild in $7 \%$, moderate in $3 \%$, moderately severe in $30 \%$, severe in $10 \%$, and profound in $2 \%$ of the ears. A positive correlation was present between the severity of hearing loss and the severity of ear hypoplasia (Spearman rank correlation coefficient 0.755 , $\mathrm{p}=<0.001$ ). Conductive hearing loss was found in $73 \%$ of the ears, mixed hearing loss in $10 \%$ and sensorineural hearing loss in $1 \%$. In $16 \%$ of the ears the type of hearing loss could not be identified. In $78 \%$ of the ears, conductive or mixed hearing loss was based on stenosis or atresia of the external auditory canal. Other causes for conductive or mixed hearing loss were middle ear effusion $(n=4)$, negative pressure $(n=2)$, or tympanic membrane perforations $(n=1)$. Hearing loss was frequently associated with ipsilateral hypoplasia of the ear or mandible, although $8 \%$ of the patients had contralateral hearing loss. Of the patients with mandibular hypoplasia and microtia, $90 \%$ had hearing loss, whereas in patients with mandibular hypoplasia and normal ears, only $33.5 \%$ had hearing loss. The authors emphasise the importance of early diagnostic hearing assessment and close audiologic follow-up in patients with CFM.

Rahbar et al. retrospectively evaluated 40 patients with CFM to evaluate the clinical, audiologic and temporal bone CT findings (12). Seven patients had bilateral CFM and 33 patients had unilateral CFM. Auricular anomalies were found in $95 \%$ of the patients, conductive hearing loss in $86 \%$ of the patients $(n=35)$ and sensorineural hearing loss in $10 \%$ of the patients $(n=4)$. Ten patients were successfully using hearing aids: five patients with unilateral CFM and five patients with bilateral CFM. The severity of conductive hearing loss in unilateral CFM was mild in $6 \%$, moderate in $3 \%$, moderately severe in $15 \%$, severe in $42 \%$, and profound in $9 \%$ of the patients. Profound sensorineural hearing loss was found in $6 \%$ of the patients with unilateral CFM. Two patients with unilateral CFM $(6 \%)$ had bilateral moderately severe conductive hearing loss. In patients with bilateral CFM, bilateral conductive hearing loss was moderately severe in $43 \%(n=3)$ and severe in $29 \%(n=2)$ patients. Chronic otitis media was reported frequently: 20 patients with unilateral CFM $(60 \%)$ and three patients with bilateral CFM $(43 \%)$ had a history of chronic otitis media. Fifteen patients with unilateral CFM (45\%) and one patient with bilateral CFM (14\%) required tympanostomy tube placement. Unilateral atresia or stenosis of the external auditory canal were found in respectively $61 \%$ and $12 \%$ of the patients with unilateral CFM and in respectively $57 \%$ and $28 \%$ of the patients with bilateral CFM. Hypoplasia or atresia of the middle ear was present in respectively $70 \%$ and $18 \%$ of the patients with unilateral CFM. All patients with bilateral CFM had hypoplastic middle ears. The ossicles were dysplastic in $73 \%$ of the patients with unilateral CFM and in $86 \%$ of the patients with bilateral CFM. The most common inner ear abnormality was a hypoplastic oval window and was found in $36 \%$ of the patients with unilateral CFM and in $29 \%$ of the patients with bilateral CFM. One unilateral and one bilateral patient had an abnormal cochlea. Other reported anomalies were: hypoplastic vestibule $(n=2)$, hypoplastic semicircular canals $(n=1)$, atretic facial nerve recess $(n=1)$, and an atretic round window $(\mathrm{n}=2)$. Patients with unilateral CFM and severe ear malformations (i.e. a high $\mathrm{E}$ of the O.M.E.N.S. score) had a significantly higher risk for inner ear anomalies (Spearman rank correlation coefficient $0.57, \mathrm{p}=<0.001$ ). Both unilateral and bilateral patients with a higher total O.M.E.N.S. score have a higher risk for inner ear malformations (Spearman rank correlation coefficient 0.47 and $0.83, p=0.006$ and $p=<0.001)$. No correlation was present between the O.M.E.N.S. score and the degree or type of hearing loss. The authors strongly recommend complete audiologic evaluation of every child with CFM, regardless of the type or severity of the facial malformations. If a CT scan for mandibular surgery is performed this should include a scan of the temporal bone to examine inner ear anomalies.

Systemic and functional difficulties in a group of eighteen patients were retrospectively studied by Strömland et al (13). Fifteen patients had hearing loss: four patients unilateral conductive hearing loss, five patients bilateral conductive hearing loss, three patients bilateral mixed hearing loss, and three patients bilateral sensorineural hearing loss. Seventeen patients had external ear malformations. Twelve patients had middle ear malformations, which were bilateral in five patients. A fusion of the malleus and incus or a hypoplastic middle ear cavity occurred most frequently, although no numbers were given. Inner ear malformations were found in three patients, which was bilateral in a single patient. All patients with inner ear malformations had a defect vestibular organ. The authors stress the importance of hearing tests in patients with CFM early in childhood that should be repeated if not conclusive. In addition, a CT-scan to evaluate the middle and inner ear and thorough otolaryngological investigation should be performed in patients with CFM.

To investigate intracranial anomalies in CFM a cross-sectional study was set up by Davide and Renzo et al (19). Thirty-five patients with CFM underwent CT imaging of the head; additional MRI of the head was performed in 32 patients. Nineteen patients fulfilled the criteria for Goldenhar syndrome which was defined by having ocular or cervical vertebral abnormalities additional to CFM and microtia. Inner ear malformations, such as atretic oval windows, abnormal facial nerve bony canal, or dysplasia of the semicircular canals, were found in $31 \%$ of the patients $(n=11)$. Inner ear abnormalities were correlated with ipsilateral seventh and eighth cranial nerve abnormalities.

In 1989, Bassila et al. retrospectively studied 50 patients with CFM to gain more knowledge of the clinical features of CFM, with special interest in facial nerve palsy and hearing loss (6). Thirtyeight unilateral and twelve bilateral patients were included. Auricular anomalies such as microtia or preauricular skin tags or pits were 
present in $92 \%$ of the patients. Facial nerve paralysis or paresis was reported in eleven patients. A total of eight patients had sensorineural hearing loss (prevalence of $16 \%$ ). In six patients the sensorineural hearing loss was accompanied by facial nerve palsy and in two patients it was not. All eight patients with sensorineural hearing loss had unilateral CFM. Interestingly, four of the eight patients with hearing loss had bilateral sensorineural hearing loss. In two patients with sensorineural hearing loss a CT scan was obtained which showed an absent cochlea and abnormal vestibulum in one patient, and a normal cochlea and vestibulum in the other patient.

The relationship between auditory and facial nerve dysfunction was assessed by Carvalho et al. too, in a retrospective cohort study of 99 patients with CFM (4). Thirty patients had bilateral CFM and 69 unilateral CFM. Auricular anomalies, including microtia and ear tags/pits, were present in 91 patients. A total of $75 \%$ of the patients $(n=74)$ had hearing loss. Fourteen of the 74 patients with hearing loss (19\%) required rehabilitation with auditory amplification. Conductive hearing loss was found in $74 \%(n=73)$ patients. Patients with auricular anomalies had conductive hearing loss twice as often $(\mathrm{p}=0.03)$. Sensorineural hearing loss was present in eleven patients, ten of these patients had conductive hearing loss too. In eight of the eleven patients, the sensorineural hearing loss was unilateral and in three patients it was bilateral. Patients with auricular anomalies had a higher incidence of sensorineural hearing loss compared to patients without auricular anomalies $(p=0.04)$. Twenty-two patients had facial nerve dysfunction (all unilateral CFM). No correlation was found between facial nerve dysfunction and sensorineural hearing loss or auricular anomalies. Patients with a more severe form of CFM or bilateral CFM were not found to have a higher risk for sensorineural hearing loss or facial nerve dysfunction. The authors strongly recommend a complete audiologic evaluation of every child in which the diagnosis of CFM is considered.

Wan et al. retrospectively studied the clinical, temporal bone and audiological findings in 70 patients with CFM (14). Seventeen patients $(24 \%)$ had bilateral CFM and 53 patients $(76 \%)$ unilateral CFM. Almost all patients (99\%) had auricular deformities. Hearing aids were used by 19 patients $(27 \%)$. A total of 87 ears were evaluated: conductive hearing loss in $82 \%$, mixed hearing loss in $15 \%$, sensorineural hearing loss in $3 \%$ of the ears. The external auditory canal was normal in $8 \%$, stenotic in $10 \%$ and atretic in $81 \%$ of the 87 ears. The middle ear was hypoplastic in $67 \%$ and atretic in $7 \%$ of the 87 ears. The ossicles were fused in $59 \%$ and absent in $23 \%$ of the 87 ears. The inner ear was abnormal in $8 \%$ of the 87 ears. The facial nerve was displaced in $40 \%$ and not identified in $15 \%$ of the 87 ears. The severity of the facial malformations, displayed by the total O.M.E.N.S. score, was correlated with the malformation on radiography of the temporal bone $(\mathrm{p}=<0.001)$. The severity and type of hearing loss was not related to the total O.M.E.N.S. score or the malformations on temporal bone radiography. This emphasises the importance of audiological testing in all patients with CFM.

A cross-sectional study of 89 patients with CFM was undertaken by Cohen et al. to study the type and frequency of maxillofacial and systemic malformations in CFM (18). All patients were evaluated for hearing impairment. External ear anomalies (microtia or anotia) were present in $83 \%$ of the patients of which $15 \%$ had bilateral ear malformations. Atresia of the auditory canal was found in $58 \%$ of the patients, and $21 \%$ of the patients had stenosis of the auditory canal. Hearing impairment, which is associated with auditory canal malformations, was present in $79 \%(n=69)$ of the patients. Bilateral deafness due to cochlea or acoustic nerve damage was found in five patients $(6 \%)$.

Cousley et al. included 50 CFM patients in a retrospective cohort study to compare two classification systems (SAT and O.M.E.N.S.) (7). Data on hearing difficulties was included in this study: $66 \%$ of the patients had hearing difficulties. Three patients had unilateral conductive hearing loss combined with bilateral sensorineural hearing loss. No further data on hearing loss or auricular anomalies was provided.

A large retrospective cohort study was set up by Heike et al. to develop a standardised approach to assess and describe facial characteristics in patients with CFM and controls (8). A total of 142 patients with CFM and 290 controls without a craniofacial syndrome were included. Sixty-seven percent $(n=91)$ of the patients with CFM and four percent $(n=9)$ were reported to have hearing difficulties. Hearing devices were used by $21 \%$ of the patients with CFM and $0.3 \%$ of the controls. No further information on hearing difficulties was available in this study.

Engiz et al. studied 31 patients with CFM to describe the phenotypic features (16). In 21 patients the hearing status was known. Eighteen of these patients had hearing loss: conductive in twelve patients, mixed- in two patients, and sensorineural hearing loss in a single patient. Three patients with an atretic ear had normal hearing in the other ear.

A cross-sectional study on hearing difficulties in a group of ten patients with CFM was performed by Goetze et al (20). All included patients had microtia. Two patients had unilateral conductive hearing loss, respectively mild and moderate. One patient had moderate bilateral conductive hearing loss. Sensorineural hearing loss was present in two patients, both bilateral. One patient had profound sensorineural hearing loss in both ears and the other patient had moderate and mild sensorineural hearing loss.

Sleifer et al. studied audiological findings in CFM in a crosssectional study (21). All ten included patients with CFM underwent tonal and vocal hearing evaluations. Conductive hearing loss was present in $40 \%$ of the left ears and $60 \%$ of the right ears. Sensorineural hearing loss was found in five ears, mild $(n=2)$, moderate $(\mathrm{n}=1)$, and profound $(\mathrm{n}=2)$. It is unclear if any patient had isolated sensorineural hearing loss. External ear malformations were found in $70 \%$ of the left ears $(n=7)$ and in $80 \%$ of the right ears $(n=8)$. No correlation was found between the severity of ear malformation and hearing loss.

To assess inner ear anomalies in Goldenhar syndrome, a retrospective review of temporal bone changes in 21 patients with Goldenhar syndrome was undertaken by Hennersdorf et al (9). Ninety percent $(n=19)$ of the patients had atresia or stenosis of the external auditory canal. Dysplasia of the ossicles was present in $90 \%$ of the patients; absence of ossicles was found in one patient (5\%). A narrowed or opacified tympanic cavity was found in $67 \%$ $(n=14)$ of the patients. The antrum mastoidium was opacified in $57 \%(n=12)$ of the cases. Thirty-three percent of the patients had inner ear anomalies of the cochlea, semicircular canals or vestibule. Cochlear hypoplasia and a common cavity deformity were both found in a single patient. Anomalies of the semicircular canals were found in five patients $(24 \%)$ and were associated with dysplasia of the vestibule, which was seen in four patients $(19 \%)$. Information on hearing difficulties was available for seven patients: four patients had isolated severe conductive hearing loss and three patients had combined hearing loss, which was correlated to severe inner ear malformations in a single patient. Interestingly, the other two patients with mixed hearing loss had no apparent inner ear malformations on imaging. The authors state that inner ear deformities are not always correlated to external and middle ear malformations, which stresses the importance of a high-resolution CT or MRI of the temporal bone in all CFM patients.

Jacobsson et al. studied the clinical appearance of syndromes that affect the structures originated from the first and second pharyngeal arches (10). This retrospective cohort study included 26 patients with unilateral CFM. Twenty-five patients had atretic external ear canals. One patient with unilateral CFM had bilateral 
middle ear deformities. Dysplasia of the ossicles was common in the studied population: malleus malformation in 25 ears, malleus fusion in three ear, incus malformation in 24 ears, incus fusion in three ears, stapes malformation in 21 ears, and a missing stapes in five ears. Two patients had a cochlear malformation, one of these patients had sensorineural hearing loss. No information on hearing in the other patients was available.

A case series of twelve patients with CFM to describe ear anomalies was initiated by Rosa et al (17). All twelve patients had external ear anomalies, eight patients had middle ear anomalies and three patients inner ear anomalies. Type of middle ear anomalies: displacement $(n=2)$, opacification $(n=2)$, and malformation $(n=2)$ of the ossicles. Agenesis of the inner ear canal was found in two patients. No information about hearing difficulties was reported.

A case series of fourteen patients with Goldenhar syndrome was presented by Bisdas et al. to study inner ear malformations in patients with Goldenhar syndrome (15). No clear criteria were used to include patients; no information on laterality of Goldenhar syndrome was available. Atresia of the external auditory canal was present unilaterally in ten patients and bilaterally in two patients. Inner ear malformations were seen in five (36\%) patients: dysplastic or absent ossicles in 13 patients, absence or dysplasia of the semicircular canals in four patients, enlarged vestibule in four patients, enlarged internal auditory canal in two patients, and a common cavity deformity in one patient. No information about hearing difficulties in these patients was provided.

Llano-Rivas et al. included 87 patients with isolated microtia and 58 patients with CFM, to study the clinical and genetic behaviour of microtia (11). A normal external auditory canal was present in $48 \%$ of the patients with CFM $(n=28) ; 47 \%$ $(n=27)$ of the patients had a unilateral atretic external auditory canal and $5 \%(\mathrm{n}=3)$ of the patients had a bilateral atretic external auditory canal. The prevalence of a normal or unilateral atretic external auditory canal was equal in patients with CFM and isolated microtia. However, patients with isolated microtia were found to have a higher prevalence of bilateral atretic external auditory canal $(16 \%$ versus $5 \%, p=0.05)$. Data on hearing loss was only available for all 145 patients and not specified for patients with CFM. Eightyfive percent of the patients had conductive hearing loss $(n=123)$. Five patients $(3 \%)$ had sensorineural hearing loss and six patients (4\%) had mixed hearing loss.

The types of ear anomalies and its prevalence found in patients with CFM are displayed in Table 1.

Table 3. Types of ear anomalies.

\begin{tabular}{llll}
\hline & Type of malformation & Prevalence & Reference \\
\hline \multirow{2}{*}{ Outer ear } & $\begin{array}{l}\text { Atresia or stenosis external } \\
\text { auditory canal }\end{array}$ & $90 \%$ & $(9)$ \\
& Atresia external auditory canal & $52 \%-96 \%$ & $(10-12,14,15,18)$ \\
& Stenosis external auditory canal & $10 \%-35 \%$ & $(12,14,18)$ \\
\hline \multirow{2}{*}{ Middle ear } & Dysplastic or absent ossicles & $93 \%$ & $(15)$ \\
& Dysplastic ossicles & $17 \%-90 \%$ & $(9,12,17)$ \\
& Agenesis ossicles & $5 \%-23 \%$ & $(9,14)$ \\
& Opacification of ossicles & $17 \%$ & $(17)$ \\
& Displacement of ossicles & $17 \%$ & $(17)$ \\
& Fusion of ossicles & $59 \%$ & $(14)$ \\
& Malleus malformation & $96 \%$ & $(10)$ \\
& Malleus fusion & $12 \%$ & $(10)$ \\
& Incus malformation & $92 \%$ & $(10)$ \\
& Incus fusion & $12 \%$ & $(10)$ \\
Stapes malformation & $81 \%$ & $(10)$ \\
Stapes aplasia & $19 \%$ & $(10)$ \\
Hypoplastic oval window & $35 \%$ & $(12)$
\end{tabular}

\begin{tabular}{llll}
\hline (continued ) & & \\
\hline Type of malformation & Prevalence & Reference \\
\hline Agenesis round window & $5 \%$ & $(12)$ \\
$\quad$ Agenesis facial nerve recess & $3 \%$ & $(12)$ \\
$\quad$ Narrowed or opacified tympanic & $67 \%$ & $(9)$ \\
$\quad$ cavity & & \\
$\quad$ Opacification of antrum & $57 \%$ & $(9)$ \\
$\quad$ mastoideum & & \\
$\quad$ Hypoplastic middle ear & $67 \%$ & $(14)$ \\
$\quad$ Agenesis middle ear & $7 \%$ & $(14)$ \\
\hline $\begin{array}{l}\text { Inner ear } \\
\quad \text { Inner ear malformations not } \\
\quad \text { specified }\end{array}$ & $8 \%-31 \%$ & $(14,19)$ \\
$\quad$ Cochlear anomaly & $5 \%-10 \%$ & $(9,12)$ \\
$\quad$ Dysplastic or absent semicircular & $3 \%-29 \%$ & $(9,12,15)$ \\
$\quad$ canals & & \\
$\quad$ Vestibule deformity & $5 \%-29 \%$ & $(9,12,15)$ \\
$\quad$ Enlarged internal auditory canal & $14 \%$ & $(15)$ \\
$\quad$ Common cavity deformity & $7 \%$ & $(15)$ \\
$\quad$ Agenesis inner ear canal & $17 \%$ & $(17)$ \\
\hline
\end{tabular}

\section{Conclusions}

\begin{tabular}{|c|c|}
\hline \multirow[t]{7}{*}{-} & Prevalence \\
\hline & $\begin{array}{l}\text { The prevalence of hearing difficulties in patients with craniofacial } \\
\text { microsomia is: }\end{array}$ \\
\hline & - Unspecified hearing loss: $\quad 50 \%-82 \%$ \\
\hline & - Conductive hearing loss: \\
\hline & - Sensorineural hearing loss: $\quad 1 \%-20 \%$ \\
\hline & - Mixed hearing loss: $\quad 6 \%-17 \%$ \\
\hline & $\operatorname{Ref}(4-8,12-14,16,18,20,21)$ \\
\hline
\end{tabular}

\begin{tabular}{l}
\hline Level $3 \quad \begin{array}{l}\text { Prevalence } \\
\text { In } 6 \%-10 \% \text { of the patients with unilateral craniofacial } \\
\text { microsomia, hearing difficulties can be present bilaterally or } \\
\text { solely on the contralateral side. }\end{array}$ \\
$\operatorname{Ref}(5,6,12)$
\end{tabular}

\begin{tabular}{|c|c|}
\hline Level 3 & $\begin{array}{l}\text { Risk factors } \\
\text { Severe microtia or anotia is positively correlated with hearing } \\
\text { loss. } \\
\text { Although a higher total O.M.E.N.S. score was found to be } \\
\text { correlated with inner ear malformation, no correlation was } \\
\text { present between the total O.M.E.N.S. score and the degree or } \\
\text { type of hearing loss. } \\
\text { No correlation was present between facial nerve dysfunction and } \\
\text { sensorineural hearing loss or auricular anomalies. However, } \\
\text { facial and vestibulocochlear nerve dysfunction were } \\
\text { correlated with ipsilateral inner ear anomalies. } \\
\text { Patients with bilateral craniofacial microsomia were not found to } \\
\text { have a higher risk for hearing difficulties compared to patients } \\
\text { with unilateral craniofacial microsomia. } \\
\text { Ref }(4,5,12,14,19)\end{array}$ \\
\hline
\end{tabular}


4.4.2 What is the policy for screening and monitoring of hearing difficulties in patients with craniofacial microsomia?

The literature search for this question was directed towards more specific questions related to the consequences and impact of screening and monitoring, the available screening tests and procedures for monitoring, and responsibilities for screening and monitoring (Appendix 1, http://links.lww.com/SCS/B697).

\section{Review of literature}

Various manuscripts included in Chapter 4.4.1. conclude that every child diagnosed with CFM should have complete audiologic evaluation $(4,5,9,11-14,20)$. Imaging of the temporal bone by using high-resolution CT or MRI to assess middle and inner ear anomalies is recommended by various authors as well $(9,12,13$, 17). Although screening is recommended, timing and frequency were not further specified.

\section{Conclusion}

No conclusions based on CFM-specific literature could be made.

\section{Considerations}

\section{- Overall quality of evidence}

Because there is no applicable literature available the recommendations are based on expert opinion with a low quality of evidence.

\section{- Balance of benefits and harms}

The steering group reasons that most parents of patients want their children to be screened for hearing difficulties, to ensure optimal speech and language development.

\section{- Outcome importance}

A delay in detecting or failure to detect hearing loss could have considerable consequences for speech, and language development (bilateral hearing loss) and/or social interaction (unilateral or bilateral hearing loss), while a hearing test is a low burden for the patient or parents. The Joint Committee on Infant Hearing emphasise the importance of early detection of hearing difficulties. A guideline for early hearing detection and intervention was published in 2007 (22).

\section{- Costs and resources}

The impact on costs and resources of the given recommendations will vary per member state, depending on the available care providers and facilities. The recommendations concern the essential requirements for adequate treatment of patients with CFM and should thus be implemented. Costs are lowest and resources are most efficiently used when care for craniofacial disorders is centralised in a limited number of expert centres per member state. A general rule that can be applied is one expert centre per 10 million inhabitants.

\section{- Inequity of the recommendation}

The goal of the European Reference Networks (ERNs) is to eliminate inequality within Europe with regard to care for patients with rare diseases. At present, not every member state offers an expert centre for CFM, or the level of provided care does not (yet) meet all the requirements that are outlined in this guideline. By defining the baseline of required care for CFM, this guideline will help these member states to reach the appropriate level. The ERN on craniofacial anomalies and ENT disorders (ERN-CRANIO) can guide a patient in Europe to the available centres of expertise (www.ern-cranio.eu) and can support care providers with diagnosis and treatment advice.

\section{- Feasibility of the recommendation}

Recommendations refer to the general requirements for delivering optimal healthcare and are discussed with members from participating European countries. Quality of care was paramount in the discussions. Centralisation is proposed as one of the core values. However, in some countries the national organisation of healthcare might impede centralisation. National implementation of the ERNs that fits the situation of each country is necessary. For the member states with the lowest number of inhabitants, the establishment of a craniofacial centre might not be feasible, and collaboration with a craniofacial centre in the surrounding countries can be an option.

\section{- Acceptability of the recommendation}

It is expected that all stakeholders want to apply and will be applying the recommendations, because they are employed in ERNacknowledged institutions. National implementation plans are necessary to ensure that recommendations fit the situation in each country. In addition, not all countries participating in the ERNCRANIO are represented in the guideline development group, and new members will join within the coming years. For these countries, acceptance and implementation of ERN guidelines such as this guideline on CFM is obligatory.

\section{Rationale of the recommendations}

All new-born infants that do not pass the neonatal hearing test should have an auditory brainstem response (ABR) test included as part of their screening. Referral to an otolaryngologist experienced in paediatric hearing loss should be made to have complete screening of both ears. Complete audiological evaluation should be done within three months of age in patients who do not pass the initial hearing test. Audiologic intervention should be initiated before the age of six months in these patients. Children who pass the neonatal hearing test but have a higher risk for hearing difficulties should be re-evaluated in 24 to 30 months. Since patients with hearing loss are at risk for a delay in speech and language development, regular monitoring for speech and language development at six months intervals should be initiated.

Screening for hearing loss will incur costs but outweighs the risk of not screening for hearing difficulties. The main aspects in determining these risks are the age of the patients and severity of CFM. Overall, all young patients in whom speech and language skills are developing should be examined by a hearing test yearly.

\section{Recommendations}

- Perform neonatal hearing test in all new-borns with craniofacial microsomia. If indicated, complete audiological evaluation in an experienced audiology centre should be performed before the age of three months to ensure timely treatment.

- Re-evaluate hearing tests in patients with craniofacial microsomia by the age of $24-30$ months.

- Regularly perform otoscopy and audiometry in patients with craniofacial microsomia including microtia and/or cleft palate by the ENT doctor/otolaryngologist.

- Audiologic intervention should be initiated before the age of six months in patients with congenital hearing loss. 


\section{Research gap}

The available literature mainly focuses on the prevalence of hearing loss but provides little information on the severity of hearing loss, types of treatment, and results of treatment.

4.4.3 What are the indications and policy for treatment of hearing difficulties in patients with craniofacial microsomia?

The literature search for this question was directed towards more specific research questions related to treatment options, symptoms, requirements, complications, and advantages and disadvantages of treatment of hearing difficulties (Appendix 1, http://links.lww.com/ SCS/B697). Unfortunately, no literature is available on the indications and policy for treatment of hearing difficulties in patients with CFM. Therefore no conclusions were written. Considerations for this question were only based on expert opinion.

\section{Considerations}

\section{- Overall quality of evidence}

Because there is no literature available the recommendations are based on expert opinion with a low quality of evidence.

\section{- Balance of benefits and harms}

The steering group reasons that most parents of patients want their children to be screened for hearing difficulties, to ensure optimal speech and language development.

\section{- Outcome importance}

Untreated hearing loss could have significant consequences for the well-being of the patient, his or her speech and language development, and his or her social participation.

\section{- Costs and resources}

The impact on costs and resources of the given recommendations will vary per member state, depending on the available care providers and facilities. The recommendations concern the essential requirements for adequate treatment of patients with CFM and should thus be implemented. Costs are lowest and resources are most efficiently used when care for craniofacial disorders is centralised in a limited number of expert centres per member state. A general rule that can be applied is one expert centre per 10 million inhabitants.

\section{- Inequity of the recommendation}

The goal of the European Reference Networks (ERNs) is to eliminate inequality within Europe with regard to care for patients with rare diseases. At present, not every member state offers an expert centre for CFM, or the level of provided care does not (yet) meet all the requirements that are outlined in this guideline. By defining the baseline of required care for CFM, this guideline will help these member states to reach the appropriate level. The ERN on craniofacial anomalies and ENT disorders (ERN-CRANIO) can guide a patient in Europe to the available centres of expertise (www.ern-cranio.eu) and can support care providers with diagnosis and treatment advice.

\section{- Feasibility of the recommendation}

Recommendations refer to the general requirements for delivering optimal healthcare and are discussed with members from participating European countries. Quality of care was paramount in the discussions. Centralisation is proposed as one of the core values. However, in some countries the national organisation of healthcare might impede centralisation. National implementation of the ERNs that fits the situation of each country is necessary. For the member states with the lowest number of inhabitants, the establishment of a craniofacial centre might not be feasible, and collaboration with a craniofacial centre in the surrounding countries can be an option.

\section{- Acceptability of the recommendation}

It is expected that all stakeholders want to apply and will be applying the recommendations, because they are employed in ERNacknowledged institutions. National implementation plans are necessary to ensure that recommendations fit the situation in each country. In addition, not all countries participating in the ERNCRANIO are represented in the guideline development group, and new members will join within the coming years. For these countries, acceptance and implementation of ERN guidelines such as this guideline on CFM is obligatory.

\section{Rationale of the recommendations}

The Joint Committee on Infant Hearing states that the initiation of early intervention for hearing loss should begin as soon as possible after the diagnosis of hearing loss but no later than the age of six months (22). Intervention before the age of six months gives significantly better outcomes on language, speech and socialemotional development compared to later-identified children. Personal amplification devices should be provided by an experienced audiologist soon after the diagnosis of hearing loss. Surgical treatment may be indicated in some patients to reconstruct malformed outer and/or middle ears and includes bone-anchored hearing aids. Cochlear implantation should be considered in patients who have limited benefits from (bone-anchored) hearing aids.

The International Microtia and Atresia Workgroup advises that all patients with microtia and external auditory atresia should be seen in a multidisciplinary team (23). It is important for patients with bilateral aural atresia to be treated with bone conduction devices or cochlear implants to support language and speech development (23). Timing of placement should be discussed with a microtia surgeon to ensure the possibility of microtia reconstruction in the future (23).

\section{Recommendations}

- Treat moderate to severe hearing loss, either with nonsurgical or surgical options.

- Coordinate surgical approach and timing in a multidisciplinary team regarding hearing augmentation and other surgical procedures including ear reconstruction and mandibular surgeries.

\section{Research gap}

No literature is available regarding indications and policy for treatment of hearing difficulties in patients with craniofacial microsomia. Availability of studies could help determine an optimal treatment strategy.

References

1. Tonn CR, Grundfast KM. What an otolaryngologist should know about evaluation of a child referred for delay in speech development. JAMA Otolaryngol Head Neck Surg. 2014; 140(3):259-65.

2. Etienne Krug AC, Shelly Chadha, Laura Sminkey, Ricardo Martinez, Gretchen Stevens, Karl White, Katrin Neumann, Bolajoko Olusanya, Paige Stringer, Mohan Kameswaran, Glyn Vaughan, Ruth Warick, Andrea Bohnert, Lillian 
Henderson, Irving Basanez, Maeva LeGeoff, Vincent Fougner, Tess Bright, Sebastian Brown. Childhood hearing loss: strategies for prevention and care. WHO Library Cataloguing-in-Publication Data. 2016.

3. Lin CY, Tseng YC, Guo HR, Lai DC. Prevalence of childhood hearing impairment of different severities in urban and rural areas: a nationwide population-based study in Taiwan. BMJ Open. 2018;8(3):e020955.

4. Carvalho GJ, Song CS, Vargervik K, Lalwani AK. Auditory and facial nerve dysfunction in patients with hemifacial microsomia. Arch Otolaryngol Head Neck Surg. 1999;125(2): 209-12.

5. Mitchell RM, Saltzman BS, Norton SJ, Harrison RG, Heike CL, Luquetti DV, et al. Hearing Loss in Children With Craniofacial Microsomia. Cleft Palate Craniofac J. 2017;54(6):656-63.

6. Bassila MK, Goldberg R. The association of facial palsy and/ or sensorineural hearing loss in patients with hemifacial microsomia. Cleft Palate J. 1989;26(4):287-91.

7. Cousley RR. A comparison of two classification systems for hemifacial microsomia. $\mathrm{Br} \mathrm{J}$ Oral Maxillofac Surg. 1993;31(2):78-82.

8. Heike CL, Wallace E, Speltz ML, Siebold B, Werler MM, Hing AV, et al. Characterizing facial features in individuals with craniofacial microsomia: A systematic approach for clinical research. Birth Defects Res A Clin Mol Teratol. 2016;106(11):915-26.

9. Hennersdorf F, Friese N, Lowenheim H, Tropitzsch A, Ernemann U, Bisdas S. Temporal bone changes in patients with Goldenhar syndrome with special emphasis on inner ear abnormalities. Otol Neurotol. 2014;35(5):826-30.

10. Jacobsson C, Granstrom G. Clinical appearance of spontaneous and induced first and second branchial arch syndromes. Scand J Plast Reconstr Surg Hand Surg. 1997;31(2):125-36.

11. Llano-Rivas I, Gonzalez-del Angel A, del Castillo V, Reyes R, Carnevale A. Microtia: a clinical and genetic study at the National Institute of Pediatrics in Mexico City. Arch Med Res. 1999;30(2):120-4.

12. Rahbar R, Robson CD, Mulliken JB, Schwartz L, Dicanzio J, Kenna MA, et al. Craniofacial, temporal bone, and audiologic abnormalities in the spectrum of hemifacial microsomia. Arch Otolaryngol Head Neck Surg. 2001;127(3):265-71.

13. Stromland K, Miller M, Sjogreen L, Johansson M, Joelsson BM, Billstedt E, et al. Oculo-auriculo-vertebral spectrum: associated anomalies, functional deficits and possible developmental risk factors. Am J Med Genet A. 2007;143A(12): 1317-25.

14. Wan J, Meara JG, Kovanlikaya A, Nelson MD, Don D. Clinical, radiological, and audiological relationships in hemifacial microsomia. Ann Plast Surg. 2003;51(2):161-6.

15. Bisdas $\mathrm{S}$, Lenarz $\mathrm{M}$, Lenarz $\mathrm{T}$, Becker $\mathrm{H}$. Inner ear abnormalities in patients with Goldenhar syndrome. Otol Neurotol. 2005;26(3):398-404.

16. Engiz O, Balci S, Unsal M, Ozer S, Oguz KK, Aktas D. 31 cases with oculoauriculovertebral dysplasia (Goldenhar syndrome): clinical, neuroradiologic, audiologic and cytogenetic findings. Genet Couns. 2007;18(3):277-88.

17. Rosa RF, Silva AP, Goetze TB, Bier Bde A, Almeida ST, Paskulin GA, et al. Ear abnormalities in patients with oculoauriculo-vertebral spectrum (Goldenhar syndrome). Braz J Otorhinolaryngol. 2011;77(4):455-60.

18. Cohen N, Cohen E, Gaiero A, Zecca S, Fichera G, Baldi F, et al. Maxillofacial features and systemic malformations in expanded spectrum Hemifacial Microsomia. Am J Med Genet A. $2017 ; 173(5): 1208-18$
19. Davide B, Renzo M, Sara G, Elisa L, Rodica M, Irene T, et al. Oculo-auriculo-vertebral spectrum: going beyond the first and second pharyngeal arch involvement. Neuroradiology. 2017;59(3):305-16.

20. Goetze TB, Sleifer P, Rosa RF, da Silva AP, Graziadio C, Zen PR. Hearing characterization in oculoauriculovertebral spectrum: A prospective study with 10 patients. Am J Med Genet A. 2017; 173(2):309-14.

21. Sleifer P, Gorsky Nde S, Goetze TB, Rosa RF, Zen PR. Audiological findings in patients with oculo-auriculo-vertebral spectrum. Int Arch Otorhinolaryngol. 2015;19(1):5-9.

22. American Academy of Pediatrics JCoIH. Year 2007 position statement: Principles and guidelines for early hearing detection and intervention programs. Pediatrics. 2007;120 (4):898-921.

23. Zhang TY, Bulstrode N, Chang KW, Cho YS, Frenzel H, Jiang $\mathrm{D}$, et al. International Consensus Recommendations on Microtia, Aural Atresia and Functional Ear Reconstruction. J Int Adv Otol. 2019;15(2):204-8.

\section{CHAPTER 4. SCREENING, MONITORING AND INDICATION FOR TREATMENT}

\subsection{Eye anomalies in craniofacial microsomia Introduction}

Originally, the triad of Goldenhar syndrome, now believed to be part of the craniofacial microsomia (CFM) 'spectrum', consisted of mandibular dysostosis, ear malformations and epibulbar dermoids (1). This illustrates the prominent role eye anomalies have in CFM. Patients with CFM regularly present with epibulbar dermoids or other eye anomalies, such as colobomata. This chapter focusses on eye anomalies in CFM. Orbital malformations will not be taken into account.

Colobomata are caused by incomplete closure of embryonic fissures and can clinically present as gaps or notches of the eyelids, lens, and/or choroid and retina. Epibulbar dermoids are benign, ectopic, congenital neoplasms that vary in composition (dermoid or lipodermoid) or location (corneal or limbal) (2). It is the most common congenital benign tumour of the eye, occurring in 1-3 per 10,000 births in the general population (3). The aetiology of epibulbar dermoids remains unclear.

Epibulbar dermoids have been classified in three grades $(4,5)$ :

Grade I: $\quad$ Superficial dermoids measuring less than $5 \mathrm{~mm}$, located on the limbus

Grade II: Limbal dermoid covering most of the cornea, down into the stroma up to Descemet's membrane, without involving the membrane.

Grade III: $\quad$ Large dermoids covering the whole cornea extending up to the pigmented epithelium of the iris.

To develop recommendations on screening, monitoring and treatment of eye anomalies in CFM the following questions were posed:

4.5.1 What is the type, prevalence and severity of eye anomalies in craniofacial microsomia?

4.5.2 What is the policy for screening and monitoring of eye anomalies in patients with craniofacial microsomia?

4.5.3 What are the indications and policy for treatment of eye anomalies in patients with craniofacial microsomia? 
An overview of the type, prevalence and severity of eye anomalies seen in patients with CFM makes it possible to develop recommendations on screening and treatment of these anomalies. Commonly seen eye anomalies in patients with CFM, such as epibulbar dermoids and colobomata, can lead to various patient relevant consequences. Limbal dermoids, including grade I dermoids, can cause amblyopia, difficulties with eyelid closure, irritation, corneal erosion, and/or aesthetic difficulties. This may be an indication for surgical resection. Other eye anomalies, as colobomata, can lead to exposure keratopathy, corneal ulceration, retinal detachment and/or cataract (6). Since timely treatment is important to maintain optimal vision, recommendations on screening and monitoring should be made.

\section{Literature search}

A systematic search of literature was performed to identify all available literature on craniofacial microsomia and synonyms. The search was conducted in Embase, Pubmed/Medline Ovid. The full search strategy is reported in the supplementary material. The inclusion and exclusion criteria are as follows:

Inclusion and exclusion criteria:

Type of studies

\section{- Original articles}

- Systematic review of sufficient quality: - The question in the systematic review matches the question of the guideline.

- The search of the systematic review was conducted in at least two relevant databases, such as the Cochrane

Library, Medline/Pubmed.

- The full search strategy was reported.

- No relevant items were missing in the search strategy.

Type of patients

- Patients with craniofacial microsomia

- Patients with epibulbar dermoids

Subject - Eye anomalies, epibulbar dermoids, coloboma

Exclusion criteria

- Original studies with $<10$ included patients

- Articles published before 1980

- Case reports

- Expert opinion

- Letters

- Editorials

Literature was screened on title and abstract. All literature on eye anomalies in CFM that was available was included in this chapter. A total of five manuscripts were included: a large retrospective cohort study by Caron et al. (7), a cross-sectional study by Cohen et al. (8), and four retrospective cohort studies by Hertle et al. (9), Ewart-Toland et al. (10), Strömland et al. (11), and Mansour et al (3).

Since no literature on the indication for treatment was available, an additional search was done. This search contained epibulbar dermoids and variants and was performed in Medline/Pubmed. Literature on indications for treatment for epibulbar dermoids in general was included in this chapter. The full search strategy is included in the supplementary material. A total of 254 articles were identified; exclusion based on title and abstract led to the inclusion of two articles. Although narrative reviews were excluded in previous chapters, a review by Pirouzian et al. (5) was included in this chapter due to the extensive nature of the review and the good link with the research question. A case series by Matsuo was included too (12).

4.5.1 What is the type, prevalence and severity of eye anomalies in craniofacial microsomia?

The literature search for this question was directed towards more specific questions related to the type (epibulbar dermoids and colobomata), prevalence and severity (mild, moderate or severe) of eye anomalies in CFM (Appendix 1, http://links.lww.com/SCS/ B697). Since this question does not relate to interventions or diagnostics, no considerations, rationale or recommendations are provided.

\section{Review of literature}

In 1985, Mansour et al. aimed to report ocular findings in a large cohort of patients with CFM (3). A total of 57 patients were included in this retrospective cohort study. Half of the patients $(51 \%)$ had ocular anomalies: $32 \%(\mathrm{n}=18)$ epibulbar (lipo)dermoids, $19 \%(n=11)$ ocular motility disorders, $12 \%(n=7)$ blepharoptosis, $11 \%(n=6)$ eyelid coloboma, and $11 \%(n=6)$ lacrimal duct or gland anomalies. Two-thirds of the epibulbar (lipo)dermoids were unilateral, the others were bilateral. All patients with an eyelid coloboma had epibulbar (lipo)dermoids too, although in one case the coloboma was located in the other eye than the (lipo)dermoid. Ocular motility disorders included esotropia in six patients, exotropia in three patients, a single patient with Duane's syndrome, a patient with abduction deficiency, and a patient with unspecified strabismus. It is unclear whether the ocular motility disorders were caused by epibulbar (lipo)dermoids. Interestingly, data on anomalies of the posterior pole of the eye was present in nineteen patients and three patients were found to have anomalies: a patient with a choroidal coloboma, one patient with a large peripapillary hypopigmentation, and a patient with a retinal vascular tortuosity.

Hertle et al. reported the ocular and adnexal findings of 49 patients with CFM (44 unilateral and 5 bilateral CFM) who all had ophthalmologic evaluation (9). Two-thirds $(67 \%)$ of the patients had some ocular or adnexal anomaly. Frequently seen ocular anomalies included: astigmatism (18\%), amblyopia (16\%) and anisometropia ( $8 \%$ ). Amblyopia was caused by multiple factors including strabismus, refractive errors, and ptosis. Another frequently seen ocular anomaly was strabismus $(22 \%, \mathrm{n}=11)$. Six of these patients had noncomitant strabismus (Duane syndrome $(\mathrm{n}=2)$, sixth nerve palsies $(\mathrm{n}=2)$ and bilateral superior oblique palsies $(n=2)$ ). Comitant exotropia was reported in three patients and esotropia in two patients. No globe or optic nerve anomalies were seen, although a single patient had tortuous retinal vasculature with normal vision in both eyes. Lid or adnexal anomalies were seen in $41 \%$ of the patients and included: dacryostenosis (15\% of all patients), blepharoptosis ( $12 \%$ of all patients), canthal dystopia $(8 \%$ of all patients), lagophthalmos ( $8 \%$ of all patients), entropion ( $4 \%$ of all patients), lipodermoids ( $4 \%$ of all patients), lid retraction ( $2 \%$ of all patients), and telecanthus ( $2 \%$ of all patients). This study shows a high frequency of ocular and adnexal anomalies in patients with CFM, which according to the authors necessitates ophthalmologic examination for all patients (9). It is important to diagnose and treat strabismus, amblyopia, and adnexal anomalies early to prevent visual loss (9).

Caron et al. reviewed 755 patients with CFM retrospectively to analyse typical phenotypes within CFM (7). Most patients had unilateral CFM $(88.6 \%)$ and $11.4 \%$ had bilateral CFM. A total of $13.4 \%$ of patients had ophthalmic anomalies such as epibulbar dermoids and/or colobomata. The location of the colobomata were 
not further specified. Patients with bilateral CFM had a significantly higher risk for ocular anomalies (Pearson $\mathrm{X} 2=27.191$, $\mathrm{p}=<0.001)$. Epibulbar dermoids were present in 81 patients (10.7\%): $74 \%$ of these patients had unilateral CFM and $26 \%$ patients had bilateral CFM. Colobomata were less frequently seen: in eleven patients $(1.5 \%)$ colobomata were present together with epibulbar dermoids and nine patients $(1.2 \%)$ solely had colobomata. No further information on ocular anomalies was reported.

Other studies reported briefly on ocular anomalies. Cohen et al. reported in a cross-sectional study of 89 patients with CFM that 41 patients (46\%) had ocular anomalies: 23 dermoid cysts $(56 \%), 15$ blepharoptosis (37\%), 13 ocular and/or eyelid coloboma (32\%), 6 dermolipoma (15\%), and 12 other eye defects (29\%) (8). A microphtalmic eye was present in 29 patients (71\%) and two patients had anophtalmia (5\%). Ewart-Toland et al. studied 21 patients with CFM (10). Two patients had epibulbar dermoids; other anomalies reported in single patients: bilateral conjunctival dermoids, corneal opacity, eye lipoma, microphthalmus, and hypoplastic tear duct. Strömland et al. retrospectively studied 18 patients with CFM (11). Eleven patients $(61 \%)$ had lipodermal dermoids of the eye and eight patients (44\%) had epibulbar dermoids. All patients with epibulbar dermoids had lipodermal dermoids too. Three patients had unilateral microphthalmus and one patient bilateral microphthalmus. Other eye anomalies that were reported: upper eyelid coloboma $(\mathrm{n}=3)$, unilateral ptosis $(\mathrm{n}=1)$, and distichiasis (two rows of eye lashes) $(\mathrm{n}=1)$.

Conclusions

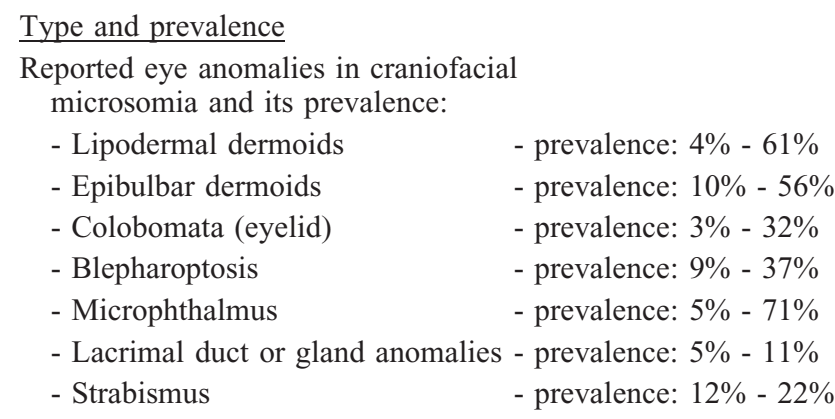

- Reported eye anomalies in CFM

in a single study

- Astigmatism

- Amblyopia

- Anisometropia

- prevalence: $18 \%$

- prevalence: $16 \%$

- prevalence: $8 \%$

- Anomalies of the posterior pole prevalence: $5 \%$

of the eye

- Anophtalmia

- prevalence: $5 \%$

Colobomata are often accompanied

by epibulbar dermoids in patients with

craniofacial microsomia.

$\operatorname{Ref}(3,7-11)$

Level 3

Prevalence

Patients with bilateral craniofacial microsomia have a significant higher risk for eye anomalies (studied in 755 patients, $\mathrm{p}=<0.001)$.

$\operatorname{Ref}(7)$

\begin{tabular}{l} 
Level $3 \quad \begin{array}{l}\text { Severity } \\
\text { Visual loss could be seen in } 8 \% \text { of the patients } \\
\text { with craniofacial microsomia, although this } \\
\text { was reported in a single study with small } \\
\text { sample size }(\mathrm{n}=49) . \\
\text { Ref }(9)\end{array}$ \\
\hline
\end{tabular}

4.5.2 What is the policy for screening and monitoring of eye anomalies in patients with craniofacial microsomia?

The literature search for this question was directed towards more specific questions related to the consequences and impact of screening and monitoring, the available screening tests and procedures for monitoring, and responsibilities for screening and monitoring (Appendix 1, http://links.lww.com/SCS/B697). Unfortunately, no literature on screening and/or monitoring of eye anomalies in patients with CFM is available.

\section{Considerations}

\section{- Overall quality of evidence}

Because there is no literature available the recommendations are based on expert opinion with a low quality of evidence.

\section{- Balance of benefits and harms}

CFM is found to be associated with a high percentage of ocular anomalies $(67 \%)$. The main concern is the risk of permanent visual impairment caused by a treatable obstacle/anomaly. Therefore, all children with CFM should be screened regarding visual development at an early stage. Persistent amblyopia causes a reduced quality of life especially, if the better eye is lost later in life and a significant (financial) burden on society. Therefore, the benefits of screening outweigh the harms.

\section{- Outcome importance}

The reduced quality of life in patients with poor visual acuity requires adequate screening, monitoring and treatment, especially if the visual acuity in the better eye is lost later in life.

\section{- Costs and resources}

The impact on costs and resources of the given recommendations will vary per member state, depending on the available care providers and facilities. The recommendations concern the essential requirements for adequate treatment of patients with CFM and should thus be implemented. Costs are lowest and resources are most efficiently used when care for craniofacial disorders is centralised in a limited number of expert centres per member state. A general rule that can be applied is one expert centre per 10 million inhabitants.

\section{- Inequity of the recommendation}

The goal of the European Reference Networks (ERNs) is to eliminate inequality within Europe with regard to care for patients with rare diseases. At present, not every member state offers an expert centre for CFM, or the level of provided care does not (yet) meet all the requirements that are outlined in this guideline. By defining the baseline of required care for CFM, this guideline will help these member states to reach the appropriate level. The ERN on craniofacial anomalies and ENT disorders (ERN-CRANIO) can guide a patient in Europe to the available centres of expertise (www.ern-cranio.eu) and can support care providers with diagnosis and treatment advice. 


\section{- Feasibility of the recommendation}

Recommendations refer to the general requirements for delivering optimal healthcare and are discussed with members from participating European countries. Quality of care was paramount in the discussions. Centralisation is proposed as one of the core values. However, in some countries the national organisation of healthcare might impede centralisation. National implementation of the ERNs that fits the situation of each country is necessary. For the member states with the lowest number of inhabitants, the establishment of a craniofacial centre might not be feasible, and collaboration with a craniofacial centre in the surrounding countries can be an option.

\section{- Acceptability of the recommendation}

It is expected that all stakeholders want to apply and will be applying the recommendations, because they are employed in ERNacknowledged institutions. National implementation plans are necessary to ensure that recommendations fit the situation in each country. In addition, not all countries participating in the ERNCRANIO are represented in the guideline development group, and new members will join within the coming years. For these countries, acceptance and implementation of ERN guidelines such as this guideline on CFM is obligatory.

\section{Rationale of the recommendations}

The main purpose of screening children with CFM is to diagnose risk factors that negatively influence visual development and provide optimal treatment. Therefore, screening of all children with CFM by an orthoptist and ophthalmologist in a specialised centre at an early stage in the visual development, i.e. age three or four, is necessary; or, in case there is an obvious anomaly obstructing the visual pathway, patients should be referred at an earlier age. Depending on the results, follow-up visits need to be scheduled on a regular basis.

Recommendations

- All patients with craniofacial microsomia should be screened at least once during the visual development (before the age of five) by an orthoptist and ophthalmologist. Depending on the results, follow-up visits need to be scheduled on a regular basis.

\section{Research gap}

No literature is available regarding the policy of screening and monitoring of eye anomalies in patients with craniofacial microsomia. Availability of studies could help to develop better screening and monitoring.

4.5.3 What are the indications and policy for treatment of eye anomalies in patients with craniofacial microsomia?

The literature search for this question was directed towards more specific research questions related to treatment options, symptoms, requirements, complications, and advantages and disadvantages of treatment of eye anomalies (Appendix 1, http://links.lww.com/SCS/ B697). Unfortunately, no literature on the indications and policy for treatment of eye anomalies in patients with CFM is available. However, since epibulbar dermoids/lipodermoids is the most commonly seen eye anomaly in patients with CFM, literature on treatment of these anomalies in non-CFM patients was included in this chapter.

\section{Review of literature}

Matsuo (12) described the process of clinical decision making in ocular dermoids or dermolipomas by retrospectively studying thirteen patients with epibulbar dermoids. Eight of the thirteen patients underwent surgical resection of the dermoids and the other five patients were observed. None of the patients had complaints of irritation or redness of the eye. The indication for surgery was not reported. Of the eight patients that had surgical resection, six patients had elevated, dome-shaped limbal dermoids and two patients had conjunctival fornix dermolipomas. No complications were seen after surgery, although all six patients that had the limbal dermoid removed showed superficial flat corneal opacification and minimal neovascularization at the place of removal. The patients and families were satisfied with the surgical outcome. The degree of postoperative astigmatism was $0-7$ diopters (median 2.9) in patients with limbal dermoids and $0-1$ diopters in the two patients with conjunctival fornix dermolipomas. Surgical resection of the limbal dermoids did not significantly influence the degree of astigmatism $(\mathrm{p}=>0.05)$. Of the five patients that did not undergo surgery, three had flat limbal dermoids, one a dome-shaped limbal dermoid, and one patient a conjunctival fornix dermolipoma. In the observation group, the patient with the dome-shaped limbal dermoids showed astigmatism. No changes in size of the dermoids was found during observation. The authors conclude that dome-shaped limbal dermoids were associated with a large degree of astigmatism and were more frequently resected, and that flat-shaped limbal dermoids and conjunctival fornix dermolipomas were not associated with astigmatism and were more frequently observed. All patients with ocular surface dermoids or dermolipomas should have a visual acuity check-up yearly. Dermoids and dermolipomas not affecting the visual axis and not impairing visual acuity can be observed at first. Surgical resection may also be indicated because of cosmetic appearance. Timing of surgery could be based on patients' preferences, although combination with other surgical procedures such as skin tag removal if indicated, should be considered in mind to reduce the risk of general anaesthesia. Ultrasound imaging could help in decision making and conducting surgery in patients with ocular dermoids that do not affect the visual axis.

A review of literature on medical and surgical management of paediatric limbal dermoids was published by Pirouzian in 2013 (5). Removal of dermoids may lead to scarring and development of pseudopterygium. Therefore, it is discouraged to remove small, asymptomatic grade I limbal dermoids. These patients should be monitored on a regular basis, once every two-three months, when the visual acuity and absence or presence of amblyopia should be examined. If the patient develops amblyopia or anisometropia, dermoid growth resulting in harm or defects of the cornea, and/ or aesthetic difficulties, surgical removal of the dermoid may be indicated. In Table 1 the indications for surgical removal of grade I limbal dermoids in children proposed by the authors are shown. For grade II or grade III limbal dermoids surgical removal is commonly indicated since these dermoids often cause amblyopia. In some dermoids a tail extending into the orbit may be present, in which case simple excision can result in perforation of the globe or incomplete resection (13). Careful evaluation by using imaging is indicated in dermoids suspected of posterior tail extension.

Table 1. Indications for surgical removal of grade I limbal dermoids, from Pirouzian (5).

Chronic eye rubbing due to irritation and recurrent conjunctivitis Amblyopia unresponsive to medical management

Progressive dellen, with corneal surface decompensation Growth and encroaching into pupillary area or optical zone Aesthetic considerations

Induction of irregular astigmatism

Inadequate lid closure 
Simple excision of corneal dermoids can lead to scar formation, persistent epithelial defects, or corneal vascularisation at the dermoid excision site, presumably due to focal limbal stem cell deficiency at the resection site. To prevent complications of simple excision, various additional techniques have been proposed. Use of autologous limbal stem cell appear to prevent complications (14). In patients with proven limbal stem cell deficiency with a partial limbal deficiency, amniotic membrane transplantation was found to be superior to allograft limbal transplantation (15). Except for two of the 31 studied eyes, all amniotic membrane-covered defects showed rapid epithelialisation and reduced inflammation, scarring and vascularisation (15). A pericardial patch graft after grade II limbal dermoid excision can be used too and is found to give good cosmetic results (16). The use of fibrin glue-assisted multi-layered amniotic membranes for reconstruction of the defect after lamellar dissection in grade I perilimbal dermoids, has been shown to be clinically successful (17). The membrane leads to volumetric filling of the defective area and should be in height with the surrounding corneal tissue. In patients with a grade III dermoid, in which the cornea and anterior chamber are involved, reconstruction of the anterior segment of the eye should be anticipated. Enucleation or evisceration with orbital reconstruction has been proposed for microphtalmic patients. A summary of the proposed treatment options is presented in Table 2 .

Table 2. Recommendations for surgical removal of ocular dermoids. From Pirouzian (5).

\begin{tabular}{ll}
\hline $\begin{array}{l}\text { Grade of paediatric limbal/ } \\
\text { corneal dermoid }\end{array}$ & Recommended techniques \\
\hline $\begin{array}{l}\text { Grade I: }<50 \mu \text { m thickness } \\
\text { and }<1 \mathrm{~mm} \text { diameter }\end{array}$ & Simple excision \\
$\begin{array}{l}\text { Grade I: }<100 \mu \mathrm{m} \text { thickness } \\
\text { and }<1 \mathrm{~mm} \text { diameter }\end{array}$ & Keratectomy + AMT + ALSCA \\
Grade II and deeper Grade I & Keratectomy + AMT + LSCA + \\
& PPG \\
& versus anterior or deep anterior \\
& lamellar keratoplasty \pm AMT \\
Grade III & Total anterior segment \\
& reconstruction \\
\hline
\end{tabular}

Abbreviations: AMT, amniotic membrane transplantation (multilayered); ALSCA, autologous limbal stem cell allograft; PPG, pericardial patch graft.

\section{Conclusions}

Level 3 Indications and Policy
Surgical resection of grade I limbal dermoids may be
indicated if complaints of eye irritation,
conjunctivitis, growth, inadequate eyelid closure,
corneal erosion, astigmatism, or aesthetic
difficulties start to occur.
Simple excision of corneal dermoids may lead to scar
formation, epithelial defects, or corneal
vascularization. Surgical resection of small,
asymptomatic grade I limbal dermoids with no
aesthetic complaints is discouraged.
Ref $(5,12)$

\begin{tabular}{l} 
Level $3 \quad \begin{array}{l}\text { Policy } \\
\text { Grade II or grade III limbal dermoids and/or dome- } \\
\text { shaped limbal dermoids lead to astigmatism more } \\
\text { frequently. Therefore, surgical resection of these } \\
\text { dermoids is commonly indicated. } \\
\operatorname{Ref}(5,12)\end{array}$ \\
\hline
\end{tabular}

\begin{tabular}{l} 
Level $32 \begin{array}{l}\text { Policy } \\
\text { Dermoids suspected of for having a tail extending into } \\
\text { the orbit should be carefully evaluated by using } \\
\text { imaging before surgical resection to avoid } \\
\text { incomplete resection or globe perforation. } \\
\operatorname{Ref}(5,12)\end{array}$ \\
\hline
\end{tabular}

\begin{tabular}{|c|c|}
\hline $\begin{array}{l}\text { Grade of paediatric limbal/ } \\
\text { corneal dermoid }\end{array}$ & Recommended techniques \\
\hline $\begin{array}{l}\text { Grade } \mathrm{I}:<50 \mu \mathrm{m} \text { thickness } \\
\text { and }<1 \mathrm{~mm} \text { diameter }\end{array}$ & Simple excision \\
\hline $\begin{array}{l}\text { Grade } \mathrm{I}:<100 \mu \mathrm{m} \text { thickness } \\
\text { and }<1 \mathrm{~mm} \text { diameter }\end{array}$ & $\begin{array}{l}\text { Keratectomy }+ \text { AMT }+ \\
\quad \text { ALSCA }\end{array}$ \\
\hline Grade II and deeper Grade I & $\begin{array}{l}\text { Keratectomy }+ \text { AMT }+ \\
\text { LSCA }+ \text { PPG } \\
\text { versus anterior or deep anterior } \\
\text { lamellar keratoplasty } \pm \\
\text { AMT }\end{array}$ \\
\hline Grade III & $\begin{array}{l}\text { Total anterior segment } \\
\text { reconstruction }\end{array}$ \\
\hline
\end{tabular}

Abbreviations: AMT, amniotic membrane transplantation (multilayered); ALSCA, autologous limbal stem cell allograft; PPG, pericardial patch graft.

$$
\text { Ref (5) }
$$

\section{Considerations \\ - Overall quality of evidence}

The quality of evidence is graded on level 3 or 4 as it is based on non-comparative studies: a case series by Matsuo et al. (12) and a narrative review by Pirouzian et al. (5). In addition, the literature is regarded as indirect as no patients with CFM were included.

\section{- Balance of benefits and harms}

The risks of removing of the dermoid should be carefully evaluated before surgical removal as even 'simple excision' can cause severe complications. Ultrasound imaging needs to be conducted in case extension posteriorly and into the orbit is suspected. When refractive errors, astigmatism and/or anisometropia, are present this should be corrected at all times with optimal spectacle correction and occlusion of the better eye in case of amblyopia. Persistent amblyopia causes a significant burden on society, financially as well as in terms of a reduced quality of life especially if the better eye is lost later in life.

\section{- Outcome importance}

The primary purpose of treatment is to achieve optimal visual outcome and cosmetic appearance. If left untreated, patients with poor visual acuity are a significant concern for society, financially as well as in terms of a reduced quality of life; especially if the visual acuity in the better eye is lost later in life.

\section{- Costs and resources}

The impact on costs and resources of the given recommendations will vary per member state, depending on the available care providers and facilities. The recommendations concern the essential 
requirements for adequate treatment of patients with CFM and should thus be implemented. Costs are lowest and resources are most efficiently used when care for craniofacial disorders is centralised in a limited number of expert centres per member state. A general rule that can be applied is one expert centre per 10 million inhabitants.

\section{- Inequity of the recommendation}

The goal of the European Reference Networks (ERNs) is to eliminate inequality within Europe with regard to care for patients with rare diseases. At present, not every member state offers an expert centre for CFM, or the level of provided care does not (yet) meet all the requirements that are outlined in this guideline. By defining the baseline of required care for CFM, this guideline will help these member states to reach the appropriate level. The ERN on craniofacial anomalies and ENT disorders (ERN-CRANIO) can guide a patient in Europe to the available centres of expertise (www.ern-cranio.eu) and can support care providers with diagnosis and treatment advice.

\section{- Feasibility of the recommendation}

Recommendations refer to the general requirements for delivering optimal healthcare and are discussed with members from participating European countries. Quality of care was paramount in the discussions. Centralisation is proposed as one of the core values. However, in some countries the national organisation of healthcare might impede centralisation. National implementation of the ERNs that fits the situation of each country is necessary. For the member states with the lowest number of inhabitants, the establishment of a craniofacial centre might not be feasible, and collaboration with a craniofacial centre in the surrounding countries can be an option.

\section{- Acceptability of the recommendation}

It is expected that all stakeholders want to apply and will be applying the recommendations, because they are employed in ERNacknowledged institutions. National implementation plans are necessary to ensure that recommendations fit the situation in each country. In addition, not all countries participating in the ERNCRANIO are represented in the guideline development group, and new members will join within the coming years. For these countries, acceptance and implementation of ERN guidelines such as this guideline on CFM is obligatory.

\section{Rationale of the recommendations}

The main purpose of treating children with CFM is to optimise visual acuity outcome and cosmetic appearance. Therefore, children with ocular disturbances need to be evaluated by a specialised orthoptist and ophthalmologist during the visual development (before the age of five). In addition, amblyopia should be treated before the age of six. Surgery has to be discussed in a multidisciplinary team, especially in the case of young children in whom vision is still developing.

\section{Recommendations}

- Children with ocular disturbances need to be evaluated by a specialised orthoptist and ophthalmologist during the visual development (before the age of five).

- Optimal spectacle correction should be provided in case of a refractive error.

- Amblyopia should be treated before the age of six.

- When surgery is considered this has to be discussed in a multidisciplinary team, carefully evaluating the harms and the benefits, especially in the case of young children in whom vision is still developing.

- Ultrasound imaging of the ocular dermoid needs to be conducted if extension posteriorly and into the orbit is suspected.

\section{Research gap}

As only indirect literature was available, future studies on eye anomalies in CFM studying treatment outcomes with long-term follow-up and patient specific outcomes, are needed. The ICHOM Craniofacial Microsomia standard set will help to create a good prospective dataset from a large number of patients with CFM (18).

\section{References}

1. Gorlin RJ, Jue KL, Jacobsen U, Goldschmidt E. Oculoauriculovertebral Dysplasia. J Pediatr. 1963;63:991-9.

2. Walker BA, Saltzman BS, Herlihy EP, Luquetti DV. Phenotypic characterization of epibulbar dermoids. Int Ophthalmol. 2017;37(3):499-505.

3. Mansour AM, Wang F, Henkind P, Goldberg R, Shprintzen R. Ocular findings in the facioauriculovertebral sequence (Goldenhar-Gorlin syndrome). Am J Ophthalmol. 1985;100(4):555559.

4. I M. Developmental Abnormalities of the Eye. Cambridge, UK: Cambridge University Press. 1937.

5. Pirouzian A. Management of pediatric corneal limbal dermoids. Clin Ophthalmol. 2013;7:607-14.

6. Schubert HD. Structural organization of choroidal colobomas of young and adult patients and mechanism of retinal detachment. Trans Am Ophthalmol Soc. 2005;103:457-72.

7. Caron C, Pluijmers BI, Wolvius EB, Looman CWN, Bulstrode $\mathrm{N}$, Evans RD, et al. Craniofacial and extracraniofacial anomalies in craniofacial microsomia: a multicenter study of 755 patients'. J Craniomaxillofac Surg. 2017;45(8):130210 .

8. Cohen N, Cohen E, Gaiero A, Zecca S, Fichera G, Baldi F, et al. Maxillofacial features and systemic malformations in expanded spectrum Hemifacial Microsomia. Am J Med Genet A. 2017;173(5):1208-18.

9. Hertle RW, Quinn GE, Katowitz JA. Ocular and adnexal findings in patients with facial microsomias. Ophthalmology. 1992;99(1):114-9.

10. Ewart-Toland A, Yankowitz J, Winder A, Imagire R, Cox VA, Aylsworth AS, et al. Oculoauriculovertebral abnormalities in children of diabetic mothers. Am J Med Genet. 2000;90(4): 303-9.

11. Stromland K, Miller M, Sjogreen L, Johansson M, Joelsson BM, Billstedt E, et al. Oculo-auriculo-vertebral spectrum: associated anomalies, functional deficits and possible developmental risk factors. Am J Med Genet A. 2007;143A (12):1317-25.

12. Matsuo T. Clinical decision upon resection or observation of ocular surface dermoid lesions with the visual axis unaffected in pediatric patients. Springerplus. 2015;4:534.

13. Bourne RA. Epibulbar dermoid tumours of the corneal limbus treated by lamellar keratoplasty. Trans Can Opthalmolog Soc. 1961;24:153-8.

14. Hong S, Kim EJ, Seong GJ, Seo KY. Limbal Stem Cell Transplantation for Limbal Dermoid. Ophthalmic Surg Lasers Imaging. 2010:1-2.

15. Tseng SC, Prabhasawat P, Barton K, Gray T, Meller D. Amniotic membrane transplantation with or without limbal allografts for corneal surface reconstruction in patients with 
limbal stem cell deficiency. Arch Ophthalmol. 1998;116(4): 431-41.

16. Lazzaro DR, Coe R. Repair of limbal dermoid with excision and placement of a circumlimbal pericardial graft. Eye Contact Lens. 2010;36(4):228-9.

17. Pirouzian A, Holz H, Merrill K, Sudesh R, Karlen K. Surgical management of pediatric limbal dermoids with sutureless amniotic membrane transplantation and augmentation. J Pediatr Ophthalmol Strabismus. 2012;49(2):114-9.

18. Measurement ICHOM. ICHOM Craniofacial Microsomia Data Collection Reference Guide. 2017.

\section{CHAPTER 4. SCREENING, MONITORING AND INDICATION FOR TREATMENT}

\subsection{Dental deformities in craniofacial microsomia \\ Introduction}

The prevalence of teeth agenesis in the general population is around $6.9 \%$ (range $1.4 \%-11.3 \%$ ), excluding third molar agenesis (1). Patients with craniofacial microsomia (CFM) are presumably at increased risk for teeth agenesis or other dental deformities. A relation with mandibular hypoplasia or other characteristics of CFM may be present.

To obtain more knowledge on dental deformities in CFM and offer potential recommendations on screening, monitoring, and treatment the following questions were posed:

4.6.1 What is the type, prevalence and severity of dental deformities in craniofacial microsomia?

4.6.2 What is the policy for screening and monitoring of dental deformities in patients with craniofacial microsomia? 4.6.3 What are the indications and policy for treatment of dental deformities in patients with craniofacial microsomia?

Awareness of dental deformities in patients with CFM is essential to identify problems in an early phase and start treatment if needed. Occlusal problems may occur due to dental anomalies, which could lead to oral health damage and/or feeding difficulties. Orthodontic treatment may be indicated to treat these deformities.

Literature search

A systematic search of literature was performed to identify all available literature on craniofacial microsomia and synonyms. The search was conducted in Embase, Pubmed/Medline Ovid. The full search strategy is reported in the supplementary material. The inclusion and exclusion criteria are as follows:

\begin{tabular}{|c|c|}
\hline Type of studies & $\begin{array}{l}\text { - Original articles } \\
\text { - Systematic review of sufficient quality: } \\
\text { - The question in the systematic review matches the } \\
\text { question of the guideline. } \\
\text { - The search of the systematic review was conducted in at } \\
\text { least two relevant databases, such as the Cochrane } \\
\text { Library, Medline/Pubmed. } \\
\text { - The full search strategy was reported. } \\
\text { - No relevant items were missing in the search strategy. }\end{array}$ \\
\hline Type of patients & - Patients with craniofacial microsomia \\
\hline Subject & - Dental anomalies \\
\hline Exclusion criteria & $\begin{array}{l}\text { - Original studies with }<10 \text { included patients } \\
\text { - Articles published before } 1980 \\
\text { - Case reports } \\
\text { - Expert opinion } \\
\text { - Letters } \\
\text { - Editorials } \\
\text { - Narrative reviews }\end{array}$ \\
\hline
\end{tabular}

Literature was screened on title and abstract. All available literature on dental anomalies in CFM was included in this chapter. A total of eight manuscripts were included: retrospective cohort studies by Ahiko et al. (2), by Farias et al. (3), by Jacobsson et al. (4), by Maruko et al. (5), and by Silvestri et al. (6), two retrospective cohort studies with a healthy control group by Seow et al. (7) and by Ongkosuwito et al. (8), and lastly a case series by Silvestri et al. (9).

4.6.1 What is the type, prevalence and severity of dental deformities in craniofacial microsomia?

The literature search for this question was directed towards more specific questions related to the type, prevalence and severity of dental deformities in CFM (Appendix 1, http://links.lww.com/SCS/B697). Since this question does not relate to interventions or diagnostics, no considerations, rationale or recommendations are provided.

\section{Review of literature}

The dental development in patients with CFM was studied by Ahiko et al. by comparing the affected side with the unaffected side (2). A total of 24 patients with unilateral CFM were included. Third molars were excluded from the analysis. Frontal and lateral cephalogram analysis showed that the included patients had a short mandibular ramus and body, a steep mandibular plane angle, large gonial angle, and a slightly convex facial structure. Orthopantomograms showed an upward canting of the occlusal plane toward the affected side in $87.5 \%$ of the patients. Eight patients $(33.3 \%)$ had a total of fourteen missing teeth. The lateral incisor of the mandible, on both the affected and unaffected side, was missing most frequently. No significant difference in tooth agenesis on the affected- versus unaffected side was present. However, a delay in tooth calcification of the first and second mandibular molars on the affected side compared to the unaffected side was found. This may be related to the mandibular hypoplasia.

Seow et al. studied dental casts of fifty patients with unilateral CFM to analyse dentitions and compared this with control subject that were matched for dental age (7). Twenty patients had casts of primary dentition $(40 \%), 15$ patients of mixed dentition $(30 \%)$ and 15 patients of permanent dentition (30\%). The mesiodistal diameter of the primary second molar and permanent first molar was found to be significantly smaller on the affected side compared to the unaffected side in patients with CFM $(\mathrm{p}<0.05)$. There were no differences in faciolingual dimensions. Patients with CFM had significantly smaller first and second molars in both maxilla and mandible compared to controls, on both the affected and unaffected side $(p<0.05)$. Although teeth on the unaffected side were smaller compared to controls, this was to a lesser extent compared to teeth on the affected side. No changes in teeth size of the anterior region of the dental arches were present.

Tooth size and morphology in patients with CFM was also studied by Farias et al (3). Plaster casts of 40 patients with CFM, age 8-21, were studied. Patients with severe CFM had significantly smaller mandibular first molars and canines on the affected side compared to patients with less severe CFM. All other teeth were not significantly different in size. The authors suspect that tooth size is closely related to the degree of mandibular hypoplasia. Also, morphological abnormalities of the mandibular first molar, such as cusp asymmetry, was seen more frequently on the affected side compared to the unaffected side $(\mathrm{p}<0.001)$, and mostly in patients with severe CFM.

Silvestri et al. retrospectively studied the incidence of teeth agenesis and failure of teeth eruption in 63 patients with CFM (6). The presence of third molars was included in the analysis for patients age $>$ eight. Almost all included patients had unilateral CFM $(n=61)$; two patients had bilateral CFM. A total of $17.4 \%$ $(n=11)$ had teeth agenesis of in total 29 teeth. All eleven patients had a tooth missing on the affected side and eight patients had a missing tooth on the contralateral side. If third molars were 
excluded from the analysis, the prevalence of teeth agenesis was $7.9 \%$, missing 13 teeth. The teeth missing most frequently was the third molar (16 of the 29), followed by the first and second premolar (7/29), and inferior lateral incisor (3/29). Agenesis of premolars appeared more frequently in patients with severe CFM. Six patients $(7.9 \%)$ had a failure of eruption of teeth, which was associated with dental agenesis in two patients.

Jacobsson et al. studied the clinical appearances of 26 patients with CFM (4). Eight patients (30.8\%) had teeth agenesis. Teeth missing most frequently were the first premolar $(n=5)$, second premolar $(n=4)$, and the third molar $(n=2)$.

Maruko et al. retrospectively studied 125 patients with CFM to describe the patterns of teeth agenesis in CFM and to compare its prevalence with healthy persons (5). Panoramic radiographs were reviewed, and teeth were reported as present if any part of the tooth bud, follicle, or tooth was visible. Third molars were excluded from the analysis. To study missing teeth in CFM, only patients age $>4$ years with presurgical panoramic radiographs were included. A total of 76 patients met these criteria. A third (32.9\%) of the 76 patients had at least one missing tooth, $17.1 \%$ had bilateral absence of teeth, $13.2 \%$ had missing teeth on the ipsilateral side of the mandibular deformity, and $2.6 \%$ of the patients had contralateral absence of teeth. Patients with severe mandibular hypoplasia had a significantly higher prevalence of teeth agenesis $(\mathrm{p}=<0.024)$. A small difference was present between the prevalence of mandibular teeth agenesis $(26 \%)$ and maxillary teeth agenesis $(24.6 \%)$. The number of missing teeth varied from one to ten; most patients $(24 \%)$ had one missing tooth. The most common missing tooth was the second premolar (11 mandibular, 5 maxillary), followed by the second molar ( 7 mandibular, 7 maxillary) and lateral incisor (6 mandibular, 4 maxillary). To determine the prevalence of teeth agenesis in CFM, only patients age $>8$ were included since by this age the enamel has formed in the permanent second molars. A total of 52 patients with CFM and 45 healthy controls were included for this analysis. The prevalence of teeth agenesis in patients with CFM was $26.9 \%$, whereas none of the controls had teeth agenesis $(\mathrm{p}=<0.001)$.

To compare the dental development of the affected and unaffected mandibular sides in patients with CFM, a retrospective cohort study with a healthy control group was initiated by Ongkosuwito et al (8). A total of 84 patients with unilateral CFM and 451 healthy controls were included. Patients with two or more missing mandibular teeth were excluded because no dental maturity score could be determined in those patients. Patients with severe mandibular hypoplasia, Pruzansky-Kaban III, showed a significant delay in dental development on the affected side $(\mathrm{p}<0.05)$. However, no significant difference in dental development between the affected and unaffected side was present in the other patients with CFM. The mean dental age was significantly younger in patients with Pruzansky-Kaban I and significantly older in patients with Pruzansky-Kaban IIb and III, compared to the healthy controls $(\mathrm{p}<0.05)$. Initially, patients with Pruzanksy-Kaban IIb/IIII do have a delay in dental development, which is followed by a catch-up in growth.

\section{Conclusions}

\section{Level 3 Prevalence and type}

Tooth agenesis

The prevalence of tooth agenesis in craniofacial microsomia varies from $8 \%$ to $33 \%$, excluding third molar agenesis, and is thus more frequent than in the general population (range $1 \%-11 \%$ ).

Most frequently missing teeth in patients with craniofacial microsomia are the first and second premolars, followed by the second molar and lateral incisor.

$\operatorname{Ref}(2,4-6)$

\begin{tabular}{|c|c|}
\hline Level 3 & $\begin{array}{l}\text { Prevalence and severity } \\
\text { Affected versus unaffected side in patients with unilateral } \\
\text { craniofacial microsomia } \\
\text { No significant difference in the prevalence of tooth agenesis } \\
\text { was found between the affected and unaffected side in } \\
\text { patients with unilateral craniofacial microsomia. } \\
\text { Patients with a Pruzansky-Kaban type III mandible deformity } \\
\text { showed a significant delay in dental development on the } \\
\text { affected side compared to the unaffected side. This } \\
\text { difference in dental development between sides was not } \\
\text { present in all other patients with craniofacial microsomia } \\
\text { (p }<0.05) \text {. } \\
\text { Patients with severe craniofacial microsomia (Pruzansky- } \\
\text { Kaban type IIb/III) showed a delay in dental development } \\
\text { compared to milder patients (Pruzansky-Kaban type I/IIa) } \\
\text { and to healthy children }(\mathrm{p}<0.05) \text {. } \\
\text { Ref }(2,5,8)\end{array}$ \\
\hline
\end{tabular}

Level 3 Severity and prevalence

Tooth size

Mandibular first molars and canines were significantly smaller in patients with severe craniofacial microsomia compared to mild craniofacial microsomia, and smaller on the affected side compared to the unaffected side $(\mathrm{p}<0.05)$. All other teeth were not significantly different in size in patients with craniofacial microsomia.

The first and second mandibular and maxillary molars on both the affected and unaffected side were found to be significantly smaller in patients with craniofacial microsomia compared to healthy controls $(\mathrm{p}<0.05)$.

Morphological abnormalities of the mandibular first molar, such as cusp asymmetry, were significantly more frequently seen on the affected side compared to the unaffected side, and mostly in patients with severe craniofacial microsomia $(\mathrm{p}<0.001)$.

$\operatorname{Ref}(3,7)$

4.6.2 What is the policy for screening and monitoring of dental deformities in patients with craniofacial microsomia?

The literature search for this question was directed towards more specific questions related to the consequences and impact of screening and monitoring, the available screening tests and procedures for monitoring, and responsibilities for screening and monitoring (Appendix 1, http://links.lww.com/SCS/B697). Unfortunately, no literature is available on the policy for screening and/or monitoring of dental deformities in patients with CFM. Therefore no conclusions were written. Considerations for this questions were only based on expert opinion.

\section{Considerations \\ - Overall quality of evidence}

Because there is no literature available the recommendations are based on expert opinion with a low quality of evidence.

\section{- Balance of benefits and harms}

Screening and monitoring for dental deformities in patients with CFM is needed as the prevalence of these deformities in these patients is high. Diagnosing dental deformities early helps make a timely treatment plan to optimise future treatment. The harms are limited as the assessment consists of intra-oral inspection and an orthopantomogram. 


\section{- Outcome importance}

Screening of outcomes through standard dental records and oral health-related quality of life questionnaires should be performed to monitor growth and treatment. Therefore a follow-up and taking orthodontic records $(5 / 6,9,12,15$ and 18 years) to evaluate growth, timing of treatment and dental deformities are needed.

\section{- Costs and resources}

The impact on costs and resources of the given recommendations will vary per member state, depending on the available care providers and facilities. Intra-oral inspection and orthopantomogram will provide a great deal of insight while the cost is relatively low. The recommendations concern the essential requirements for adequate treatment of patients with CFM and should thus be implemented. Early diagnosis by means of an orthopantomogram to diagnose dental malformations should be done; this diagnostic tool is widely available and at relatively low cost. In the case that a CBCT is taken for other diagnostic reasons, the CBCT should be sufficient and no additional OPT should be taken. However, the use of 3D stereophotogrammetry brings additional costs. Costs are lowered and resources are most efficiently used when care for craniofacial disorders is centralised in a limited number of expert centres per member state. A general rule that can be applied is one expert centre per 10 million inhabitants.

\section{- Inequity of the recommendation}

The goal of the European Reference Networks (ERNs) is to eliminate inequality within Europe with regard to care for patients with rare diseases. At present, not every member state offers an expert centre for CFM, or the level of provided care does not (yet) meet all the requirements that are outlined in this guideline. By defining the baseline of required care for CFM, this guideline will help these member states to reach the appropriate level. The ERN on craniofacial anomalies and ENT disorders (ERN-CRANIO) can guide a patient in Europe to the available centres of expertise (www.ern-cranio.eu) and can support care providers with diagnosis and treatment advice.

\section{- Feasibility of the recommendation}

Recommendations refer to the general requirements for delivering optimal healthcare and are discussed with members from participating European countries. Quality of care was paramount in the discussions. Centralisation is proposed as one of the core values. However, in some countries the national organisation of healthcare might impede centralisation. National implementation of the ERNs that fits the situation of each country is necessary. For the member states with the lowest number of inhabitants, the establishment of a craniofacial centre might not be feasible, and collaboration with a craniofacial centre in the surrounding countries can be an option.

\section{- Acceptability of the recommendation}

It is expected that all stakeholders want to apply and will be applying the recommendations, because they are employed in ERNacknowledged institutions. National implementation plans are necessary to ensure that recommendations fit the situation in each country. In addition, not all countries participating in the ERNCRANIO are represented in the guideline development group, and new members will join within the coming years. For these countries, acceptance and implementation of ERN guidelines such as this guideline on CFM is obligatory.

\section{Rationale of the recommendations}

In order to diagnose dental deformities, an intra oral inspection should be combined with the assessment of an orthopantomogram preferably by an orthodontist and/or a special care dentist who has affinity with craniofacial malformations. Since these dental deformities including growth may alter over time, the craniofacial orthodontist needs to do a follow-up over time. Both the first screening and follow-up should be done in a multidisciplinary team setting. Standard dental records should be obtained for orthodontic management. Screening of outcomes through standard dental records and oral health-related quality of life questionnaires should be performed to monitor growth and treatment. Therefore a followup and taking orthodontic records $(5-6,9-10,12,15$ and $17-18$ years) to evaluate growth, timing of treatment and dental deformities are needed. Screening should start by the age of six as at this age eruption of the first molars occur.

Patients with craniofacial deformities, including CFM, should have routine dental care, as these patients are at increased risk for dental decay.

Recommendations

- Patients with craniofacial microsomia should have routine dental care.

- Patients with craniofacial microsomia should be seen from age five by an orthodontist within a multidisciplinary team to diagnose dental deformities.

- Perform screening for dental deformities by intra-oral inspection and standard dental records.

- Take orthodontic records in a structured schedule, at 5$6,9-10,12,15$ and $17-18$ years of age.

\section{Research gap}

Research should focus on following CFM patients with 3D examinations to determine whether progressiveness is a problem for growth over time. To understand growth and other dental deformities in CFM patients better, research should at least use a design that involves a control group. CFM does not involve the mandible only, therefore research should also look into the complete craniofacial complex. The exact impact on having additional dental malformations in CFM patients is unclear compared to CFM patients that don't have these additional dental malformations. Therefore, a component should be included in any oral healthrelated quality of life research in CFM patients related to the dental malformations described above.

The ICHOM initiative has produced a manual for specific data collection for CFM patients. In these patients they also recommend gathering quality of life data. However, much work is still to be done and it would be worthwhile to investigate which parts of the overall treatment have the most impact on the OHrQol of CFM patients. This can be combined with taking the orthodontic records.

4.6.3 What are the indications and policy for treatment of dental deformities in patients with craniofacial microsomia?

The literature search for this question was directed towards more specific research questions related to treatment options, symptoms, requirements, complications, and advantages and disadvantages of treatment of dental deformities (Appendix 1, http://links.lww.com/ SCS/B697). Only one study that reported on treatment options for dental deformities in CFM and met the inclusion criteria was found in the literature. No literature based conclusions on the policy for treatment of dental deformities could be drawn.

\section{Review of literature}

Silvestri et al. presented a case series of sixteen children with unilateral CFM who were treated with an asymmetrical functional activator (AFA) (9). Before and after therapy, electromyography 
was performed to evaluate neuromuscular deficits of the masticatory muscles on the affected and unaffected sides. Treatment outcomes were evaluated based on panoramic radiographs. Functional therapy, by using an AFA, gave additional mandibular and maxillary growth on the affected side and increased neuromuscular function in seven patients with mild CFM. No additional surgery was needed in these patients. Another five patients with moderate CFM underwent functional therapy followed by conventional orthodontics which induced a growth response. Four of these patients required surgical mandibular repositioning around the age of ten. In another four patients with severe CFM, functional therapy and orthodontic treatment with fixed appliances was initiated to prepare for surgical intervention around the age of ten. The authors conclude that it is possible to induce balanced maxillamandibular growth by using an AFA in children with unilateral CFM. In mild cases, the use of an AFA can induce mandibular growth. Additional orthodontic treatment with fixed appliances can be used to perfect the occlusion. In patients with severe CFM, the use of an AFA is limited. Nevertheless, the authors advocate the use of an AFA in these patients too, to correct dento-alveolar disorders and prepare the neuromuscular system for the new spatial position after surgery. The aim of functional therapy in patients with severe CFM is to lay the basis for surgery and not to stimulate growth.

Conclusions

Level 3 Indications
Dentofacial orthopaedic treatment has been proposed but due
to study limitations no conclusions on mandibular growth
effect can be drawn.
Ref (9)

\section{Considerations \\ - Overall quality of evidence}

The conclusion on treatment with an asymmetrical functional activator was based on a single case series, which studied a very small patient sample $(n=16)(9)$. The reported outcome of treatment, mandibular growth, can be questioned since it was based on comparison of panoramic radiographs. The duration of treatment is thus unknown. The quality of evidence is graded on level 3 because the conclusion was based on a non-comparative study, but is considered to be very weak. No conclusions on the indications and policy for treatment of dental deformities in CFM can be drawn.

\section{- Balance of benefits and harms}

Treatment of dental deformities leads to a better occlusal relationship and is therefore beneficial for the patient. Some patients might require treatment with an activator, which may have a negative influence on the quality of life of the patient, although this hasn't been studied in patients with CFM.

\section{- Outcome importance}

Treatment of dental deformities may be needed to achieve good oral health in the patient and should always be discussed in a multidisciplinary team. It is important to distinguish between a functional and a non-functional condyle or mild versus severe CFM. In mild cases an activator treatment can be started in dialogue with patient and parents. In more severe cases treatment planning should aim to get congruent arches by orthodontic treatment trying to avoid later surgical expansion or implant placement when orthodontic closure would have been an option in collaboration with the surgeon.

\section{- Costs and resources}

The impact on costs and resources of the given recommendations will vary per member state, depending on the available care providers and facilities. The recommendations concern the essential requirements for adequate treatment of patients with CFM and should thus be implemented. Costs are lowest and resources are most efficiently used when care for craniofacial disorders is centralised in a limited number of expert centres per member state. A general rule that can be applied is one expert centre per 10 million inhabitants.

\section{- Inequity of the recommendation}

The goal of the European Reference Networks (ERNs) is to eliminate inequality within Europe with regard to care for patients with rare diseases. At present, not every member state offers an expert centre for CFM, or the level of provided care does not (yet) meet all the requirements that are outlined in this guideline. By defining the baseline of required care for CFM, this guideline will help these member states to reach the appropriate level. The ERN on craniofacial anomalies and ENT disorders (ERN-CRANIO) can guide a patient in Europe to the available centres of expertise (www.ern-cranio.eu) and can support care providers with diagnosis and treatment advice.

\section{- Feasibility of the recommendation}

Recommendations refer to the general requirements for delivering optimal healthcare and are discussed with members from participating European countries. Quality of care was paramount in the discussions. Centralisation is proposed as one of the core values. However, in some countries the national organisation of healthcare might impede centralisation. National implementation of the ERNs that fits the situation of each country is necessary. For the member states with the lowest number of inhabitants, the establishment of a craniofacial centre might not be feasible, and collaboration with a craniofacial centre in the surrounding countries can be an option.

\section{- Acceptability of the recommendation}

It is expected that all stakeholders want to apply and will be applying the recommendations, because they are employed in ERNacknowledged institutions. National implementation plans are necessary to ensure that recommendations fit the situation in each country. In addition, not all countries participating in the ERNCRANIO are represented in the guideline development group, and new members will join within the coming years. For these countries, acceptance and implementation of ERN guidelines such as this guideline on CFM is obligatory.

\section{Rationale of the recommendations}

Since the CFM phenotype is so highly variable, it is very difficult to generalise a study over the whole CFM patient group. Therefore it is advised to use a classification system like the Pruzansky/Kaban or the O.M.E.N.S. Clinicians can then distinguish between cases that have a functional condyle at the affected side versus a non-functional or absent condyle. In very mild cases in which no surgical correction is expected to be necessary at a later age, mild corrections may be gained by an activator. However, the activator should then be activated in a sagittal direction but also in a cranial-caudal direction. The last direction is best addressed during the largest development of the jaws. This is the period in which permanent teeth erupt and can gain height. This also means a long treatment duration with the activator and may be less (cost) efficient in more severe cases. Therefore this should only be done in mild cases, while more severe cases profit more from not providing 
activator treatment, when surgical correction may be necessary in the future. Whether long-duration activator treatment or a policy of 'wait and see' may have a different outcome in terms of OHrQol remains to be answered. Another reason not to use a prolonged activator treatment is that the development of soft tissue is questionable in CFM cases and may affect speech. Additionally, it is important not to lose cooperation of the patient due to prolonged treatment before the moment you really need cooperation. In both mild and severe cases, the solution and planning to solve the problem of dental malformations should be determined early and a clear treatment goal should be described.

The most important aspect is avoiding use of orthodontic resources and the risk of overtreatment and thereby loss of cooperation in the process. Interaction with all members of the multidisciplinary team is needed to provide the best overall treatment.

\section{Recommendations}

- Dentofacial orthopaedic treatment can be considered appropriate in very mild craniofacial microsomia cases. In severe craniofacial microsomia patients, current evidence does not promote activator treatment.

- Orthodontic treatment should be discussed and coordinated in a multidisciplinary team depending on the decision to conduct orthognathic surgery or not.

\section{Research gap}

The ICHOM initiative has produced a manual for specific data collection for CFM patients. In these patients they also recommend gathering quality of life data. However, much work is still to be done and it would be worthwhile to investigate which parts of the overall treatment have the most impact on the OHrQol of CFM patients. This can be combined with taking the orthodontic records.

\section{References}

1. Takehiko Shimizu TM. Prevalence and genetic basis of tooth agenesis. Japanese Dental Science Review. 2009;45(1):52-8.

2. Ahiko N, Baba Y, Tsuji M, Suzuki S, Kaneko T, Kindaichi J, et al. Investigation of maxillofacial morphology and dental development in hemifacial microsomia. Cleft Palate Craniofac J. 2015;52(2):203-9.

3. Farias M, Vargervik K. Tooth size and morphology in hemifacial microsomia. Int J Paediatr Dent. 1998;8(3):197-201.

4. Jacobsson C, Granstrom G. Clinical appearance of spontaneous and induced first and second branchial arch syndromes. Scand J Plast Reconstr Surg Hand Surg. 1997;31(2):125-36.

5. Maruko E, Hayes C, Evans CA, Padwa B, Mulliken JB. Hypodontia in hemifacial microsomia. Cleft Palate Craniofac J. 2001;38(1):15-9.

6. Silvestri A, Natali G, Fadda MT. Dental agenesis in hemifacial microsomia. Pediatr Dent. 1996;18(1):48-51.

7. Seow WK, Urban S, Vafaie N, Shusterman S. Morphometric analysis of the primary and permanent dentitions in hemifacial microsomia: a controlled study. J Dent Res. 1998;77(1):27-38.

8. Ongkosuwito EM, de Gijt P, Wattel E, Carels CE, KuijpersJagtman AM. Dental development in hemifacial microsomia. J Dent Res. 2010;89(12):1368-72.

9. Silvestri A, Natali G, Iannetti G. Functional therapy in hemifacial microsomia: therapeutic protocol for growing children. J Oral Maxillofac Surg. 1996;54(3):271-8; discussion 8-80.

\section{CHAPTER 4. SCREENING, MONITORING AND INDICATION FOR TREATMENT}

\subsection{Vertebral anomalies in craniofacial microsomia \\ Introduction}

The prevalence of vertebral anomalies in the general population is considered to be $0.1 \%$ to $2 \%(1,2)$. In patients with craniofacial microsomia (CFM) these anomalies are more frequently observed. Originally, patients with the facial characteristics of CFM and vertebral anomalies were considered to have Goldenhar syndrome or oculo-auriculo-vertebral spectrum/disorder. These syndromes are now considered to be a variant of CFM. Various types of vertebral anomalies have been reported in patients with CFM, such as hemivertebrae, blockvertebrae, or scoliosis (3-5).

To obtain more knowledge on vertebral anomalies in CFM and offer potential recommendations on screening, monitoring and treatment the following questions were posed:

4.7.1 What is the type, prevalence and severity of vertebral anomalies in craniofacial microsomia?

4.7.2 What is the policy for screening and monitoring of vertebral anomalies in patients with craniofacial microsomia?

4.7.3 What are the indications and policy for treatment of vertebral anomalies in patients with craniofacial microsomia?

Vertebral anomalies may have several negative consequences and be present without symptoms $(6,7)$. Dysplastic cervical vertebrae can lead to cervical spine instability (6). Manipulation of the cervical spine in patients with cervical spine instability can cause compression of the spinal cord or vertebral artery, which can have various neurological consequences (7-9). Another risk of vertebral anomalies is the development of progressive scoliosis or fractures of ankylosed vertebrae. This may be caused by incorrect formation or fusion of vertebrae (6). Progressive scoliosis should be prevented in patients with early-onset scoliosis. This type of scoliosis occurs before the age of ten $(10,11)$. It is relevant to optimise the development of the spine, chest wall and lungs, which could potentially be achieved by surgical treatment. Early identification and treatment of vertebral anomalies is relevant to prevent these potential harms.

\section{Literature search}

A systematic search of literature was performed to identify all available literature on craniofacial microsomia and synonyms. The search was conducted in Embase, Pubmed/Medline Ovid. The full search strategy is reported in the supplementary material. The inclusion and exclusion criteria are as follows:

Inclusion and exclusion criteria:

Type of studies - Original articles

- Systematic review of sufficient quality:

- The question in the systematic review matches the question of the guideline.

- The search of the systematic review was conducted in at least two relevant databases, such as the Cochrane Library, Medline/Pubmed.

- The full search strategy was reported.

- No relevant items were missing in the search strategy.

Type of patients - Patients with craniofacial microsomia Subject - Vertebral anomalies 


\author{
Exclusion criteria - Original studies with $<10$ included patients \\ - Articles published before 1980 \\ - Case reports \\ - Expert opinion \\ - Letters \\ - Editorials \\ - Narrative reviews
}

Literature was screened on title and abstract. All literature available on vertebral anomalies in CFM was included in this chapter. A retrospective cohort study on craniofacial and extracraniofacial anomalies in CFM by Caron et al. (12) was excluded because the same cohort was studied by Renkema et al. (13), with more detail on vertebral anomalies. A total of six manuscripts were included, five on the prevalence and types of vertebral anomalies and one article on treatment: a cross-sectional study by Cohen et al. (14), a systematic review by Renkema et al. (15), and four retrospective cohort studies by Pegler et al. (16), Davide et al. (17), Renkema et al. (13), and Connell et al. (18).

\subsubsection{What is the type, prevalence and severity of vertebral} anomalies in craniofacial microsomia?

The literature search for this question was directed towards more specific questions related to the type (block vertebrae, hemivertebrae, scoliosis, and rib anomalies), prevalence and severity of vertebral anomalies in CFM (Appendix 1, http://links.lww.com/ SCS/B697). Since this question does not relate to interventions or diagnostics, no considerations, rationale or recommendations are provided.

\section{Review of literature}

The prevalence and type of vertebral anomalies in CFM reported in literature was examined in a systematic review by Renkema et al. (15). A systematic search of literature was performed and studies on patients with isolated microtia or case-reports were excluded. A total of 31 articles were included in this review: 26 articles described the prevalence of vertebral anomalies in CFM and 22 articles the type of vertebral anomalies in CFM. In two of the 31 included studies fewer than ten patients were analysed; both studies only assessed the type of vertebral anomalies in CFM. The prevalence of vertebral anomalies in CFM varied from $8 \%$ to $79 \%$. This large variability was due to differences in sample size, study characteristics, patient selection and the level of spine investigation. Studies with larger sample size tended to show prevalences around $25 \%$. Vertebral anomalies were most frequently reported in the cervical and thoracic spine and were less often seen in the lumbar spine. Hemivertebrae, block vertebrae and scoliosis were most often described. Rib anomalies, such as fusion, aplasia, hypoplasia, or extra ribs were commonly seen and reported in ten studies. Some studies found a higher prevalence of additional extracraniofacial anomalies in other tracts in patients with CFM and vertebral anomalies $(19,20)$. Patients with CFM and cervical spine anomalies also had lower cognitive, fine motor, and expressive language scores (21). A more severe facial deformity, by using the sum of the O.M.E.N.S. score, increased the risk for the presence of extracraniofacial, and thus vertebral, anomalies (22). The authors conclude that vertebral anomalies are present in a substantial proportion of patients with CFM. To prevent harmful consequences, careful and extensive physical and neurological examination of each patient with CFM is indicated to diagnose these anomalies at an early stage.

Four studies on vertebral anomalies in CFM were published after publication of the systematic review. Davide et al. found a prevalence of $40 \%$ of vertebral anomalies in 35 studied patients (17). No further information on these anomalies was provided. Cohen et al. reported a prevalence of $47 \%(n=40)$ of vertebral anomalies in their studied cohort of 89 patients (14). The type of vertebral anomalies was hemivertebrae $(n=20)$, scoliosis $(n=18)$, rib anomalies $(n=10)$, vertebral fusion/block vertebrae $(n=9)$, butterfly vertebrae $(n=8)$, vertebral schisis $(n=8)$, and spina bifida $(n=3)$. No correlation between the severity of extracraniofacial anomalies and facial deformities by using the O.M.E.N.S. score was present. Pegler et al. studied 41 patients with CFM and $65.9 \%(n=27)$ had vertebral anomalies (16). Similar vertebral anomalies were reported: scoliosis $(n=13)$, hemivertebrae $(n=10)$, block vertebrae $(n=9)$, incomplete fusion of vertebral arches $(n=4)$, sacral dimple $(n=5)$, spina bifida $(n=4)$, and transitional vertebrae $(n=4)$.

A large, multicentre, retrospective cohort study aiming to study the prevalence, types and symptoms of vertebral anomalies in CFM was performed by Renkema et al. (13). Patients with isolated microtia were excluded in this study. A total of 991 patients were included. Vertebral anomalies were present in $28 \%(n=275)$ of the patients. Patients with bilateral CFM had a higher prevalence of vertebral anomalies compared to unilateral patients (Pearson $\chi^{2}$ $(\mathrm{df1})=15.93$, odds ratio $=2.21, \mathrm{p}=<0.001)$. Furthermore, patients with a more severe facial deformity had a higher risk for vertebral anomalies. A correlation between the Orbit, Mandible, Nerve, and Soft tissue scores on the O.M.E.N.S. scale and the presence of vertebral anomalies was found. The Mandible score on the O.M.E.N.S. scale was based on the Pruzansky-Kaban score in this study. Vertebral anomalies were present in all sections of the spine: cervical spine $(n=158)$, thoracic spine $(n=187)$, lumbar spine (77), and/or in multiple segments of the spine $(n=131)$. The anomalies reported most frequently were block vertebrae, hemivertebrae, rib anomalies, and scoliosis. The curvature of the scoliosis extended to multiple segments in 83 patients. Specific anomalies of the cervical spine such as cervical ribs $(n=12)$, cervical spine instability $(n=7)$, occipitalisation of the atlas $(n=6)$, rotated subluxation of C1-C2 $(n=4)$, or os odontoideum $(n=2)$ were seen in multiple patients. Clinical symptoms of vertebral anomalies were present in $44 \%(n=122)$ of the 275 patients with vertebral anomalies. The symptoms most frequently reported were torticollis $(n=40)$, back or neck pain $(n=28)$, and limited neck movement $(n=22)$. These symptoms were most frequently reported by patients with vertebral anomalies, but also by some patients without vertebral anomalies. Besides vertebral anomalies, other extracraniofacial anomalies were often reported in the studied cohort. A total of 462 (47\%) patients had extracraniofacial anomalies. Of the patients with vertebral anomalies, $61 \%$ had additional extracraniofacial anomalies in other tracts. A significantly higher risk for extracraniofacial anomalies in patients with vertebral anomalies was found for almost all tracts: central nervous system (odds ratio $=4.46$ ), spinal cord anomalies (odds ratio $=77.84)$, cardiac anomalies (odds ratio $=3.01$ ), gastrointestinal anomalies (odds ratio $=3.67$ ), and urogenital anomalies (odds ratio $=3.41$ ). Although the prevalence of pulmonary anomalies was higher in patients with vertebral anomalies and a trend towards significance could be observed, no statistically significant difference was found (odds ratio $=2.17, \mathrm{p}=0.055$ ). The authors advised to screen all patients with CFM for vertebral anomalies by performing physical and neurological examination. Patients suspected of having vertebral anomalies or patients with a higher risk for vertebral anomalies should be assessed by using standard upright posterior-anterior and lateral radiographs. Flexion-extension radiographs of the cervical spine should be made in patients with cervical spine anomalies to rule out cervical spine instability. Since patients with vertebral anomalies are at increased risk for having additional extracraniofacial anomalies, cardiac, renal, and neurological evaluations are indicated in patients with vertebral anomalies, according to the authors. 


\section{Conclusions}

\section{Prevalence}

The prevalence of vertebral anomalies in

craniofacial microsomia is $8-79 \%$

presumably approximating $28 \%$.

$\operatorname{Ref}(13-17)$

\section{Considerations \\ - Overall quality of evidence}

Because there is no literature available the recommendations are based on expert opinion with a low quality of evidence.

\section{- Balance of benefits and harms}

To the best of our knowledge evidence on the harms and benefits of screening for vertebral anomalies do not exist. Some evidence on the harms of screening for adolescent idiopathic scoliosis is available. It has been reported that false-positive screening may cause important potential harms, including unnecessary surgery or bracing. Rates of potential harms are reported from $0.8 \%$ to $21.5 \%$ (23). The direct harms of screening are subject to debate. Potential direct harms of false-positive results include multiple unnecessary followup visits, unnecessary radiation exposure with an increased cancer risk, overtreatment and exposure treatment-related (iatrogenic) risks, or psychosocial effects including hospitalisation and the diagnosis of clinically non-significant scoliosis (23). Children, especially those younger than five years, are not only more prone to radiation-induced malignancies due to increased radio-sensitivity of certain organs but also due to the fact that they have a longer latency period to develop a cancer (24). The use of CT for paediatric spine should be limited because of the $50 \%$ increase in mean radiation dose to the spine in paediatric patients relative to conventional radiography (25). It has been reported that lifetime cancer mortality risks attributable to the radiation exposure from a CT of the cervical spine for a one year old is approximately 0.07 to $0.18 \%$ which is much higher than that for adults who are exposed to a CT of the cervical spine (26).

\section{- Outcome importance}

The main reason to perform screening of the spine in patients with CFM is the early detection of spine anomalies and to prevent scoliosis and/or neurological deficits including spinal cord injury. In patients who are asymptomatic, neurological and radiological imaging should be performed. Patients who develop symptoms should be referred to a neurologist or orthopaedic surgeon.

\section{- Costs and resources}

The impact on costs and resources of the given recommendations will vary per member state, depending on the available care providers and facilities. The recommendations concern the essential requirements for adequate treatment of patients with CFM and should thus be implemented. Costs are lowest and resources are most efficiently used when care for craniofacial disorders is centralised in a limited number of expert centres per member state. A general rule that can be applied is one expert centre per 10 million inhabitants.

\section{- Inequity of the recommendation}

The goal of the European Reference Networks (ERNs) is to eliminate inequality within Europe with regard to care for patients with rare diseases. At present, not every member state offers an expert centre for CFM, or the level of provided care does not (yet) meet all the requirements that are outlined in this guideline. By defining the baseline of required care for CFM, this guideline will help these member states to reach the appropriate level. The ERN on craniofacial anomalies and ENT disorders (ERN-CRANIO) can guide a patient in Europe to the available centres of expertise (www.ern-cranio.eu) and can support care providers with diagnosis and treatment advice. 


\section{- Feasibility of the recommendation}

Recommendations refer to the general requirements for delivering optimal healthcare and are discussed with members from participating European countries. Quality of care was paramount in the discussions. Centralisation is proposed as one of the core values. However, in some countries the national organisation of healthcare might impede centralisation. National implementation of the ERNs that fits the situation of each country is necessary. For the member states with the lowest number of inhabitants, the establishment of a craniofacial centre might not be feasible, and collaboration with a craniofacial centre in the surrounding countries can be an option.

\section{- Acceptability of the recommendation}

It is expected that all stakeholders want to apply and will be applying the recommendations, because they are employed in ERNacknowledged institutions. National implementation plans are necessary to ensure that recommendations fit the situation in each country. In addition, not all countries participating in the ERNCRANIO are represented in the guideline development group, and new members will join within the coming years. For these countries, acceptance and implementation of ERN guidelines such as this guideline on CFM is obligatory.

\section{Rationale of the recommendations}

In CFM a variety of structural developmental anomalies of the vertebral column may occur (block vertebrae, hemivertebrae, scoliosis including rib anomalies). Often, these anomalies are simple with minimal clinical consequence, but sometimes they are or may become complex with structural and neurologic implications.

For screening and monitoring of vertebral anomalies we have to consider some general aspects of the normal spine. It is known that children under the age of eight are more susceptible to injury to the craniocervical junction $(\mathrm{C} 0-\mathrm{C} 2)$ due to specific features of their anatomic development (27). First of all the paediatric body is relatively smaller than the head which may increase the force moment on the CVJ in trauma. Secondly, the cervical musculature is not strong enough to stabilise the head appropriately. Thirdly, due to the increased laxity of the ligaments there is a greater mobility of the upper cervical spine as compared to the spinal cord. Hence spinal cord injury can occur without radiographic abnormalities (SCIWORA). For screening of vertebral abnormalities in CFM patients knowledge about the normal age-specific anatomy is important to distinguish these anomalies from trauma (bipartite atlas versus Jefferson fracture, butterfly vertebra versus burst fracture) especially in acute situations.

Almost half of the patients with CFM show symptoms of vertebral anomalies (13). Trenga et al. have shown a high prevalence of cord abnormalities associated with congenital spinal anomalies (28). Approximately 55\% of children with congenital bony spinal deformities are associated with intraspinal neural axis defects. From these two point estimates we may conclude that one out of four CFM patients will have spinal cord anomalies. Trenga et al recommend to order MRIs of the brainstem and spinal cord for all patients with congenital vertebral malformation. The timing of the MRI remains controversial. Due to the high incidence of underlying neural defects, all patients with congenital spinal deformities should have regular examinations including a neurological survey. For screening of vertebral anomalies in CFM no consensus exists on when, how and why screening should be performed. For adolescent idiopathic scoliosis existing screening guidelines are available (29). To summarise, these guidelines recommend visual inspection of the spine to look for asymmetry of the shoulders, shoulder blades, and hips. In the United States, the forward bend test is commonly used to screen for idiopathic scoliosis. The clinician visually inspects the spine of a patient while the patient is standing upright. Next, the patient stands with feet together and bends forward at the waist with arms hanging and palms touching while visual inspection is repeated. A scoliometer, which measures the angle of trunk rotation, may be used during the forward bend test. An angle of trunk rotation of $5^{\circ}$ to $7^{\circ}$ is often the threshold for referral for radiography to confirm the diagnosis and to quantify the Cobb's angle.

Screening questions and clinical examinations related to neck/ back symptoms should be undertaken at initial consultation and as part of pre-operative workup. Patients should be referred appropriately and attention should be payed when patients are undergoing general anaesthesia.

\section{Recommendations}

- Screening questions and clinical examinations related to neck/back symptoms should be undertaken at initial consultation and as part of pre-operative workup.

- All patients with craniofacial microsomia who have neurologic symptoms (e.g., paraesthesia, numbness, or weakness) or neck pain suggestive of neuronal injury should be evaluated as soon as possible by a (paediatric) neurologist.

- Patients should be referred appropriately and attention to the cervical spine should be payed when patients are undergoing general anaesthesia.

\section{Research gap}

No literature on screening anomalies in patients with CFM is available. Since the incidence of CFM is rare with the knowledge that not every CFM patient has vertebral anomalies, prospective randomised controlled screening studies are difficult but also maybe unethical to perform. Controlled trials that allow for comparison of screened and non-screened patients will provide insight on the effect of screening but are likely to be unethical. Studies on the potential harms of screening and treatment are also needed and more feasible to perform. Registries with information on long-term outcomes would be helpful. For idiopathic scoliosis, a study regarding benefits and harms has been published. The study by the US Preventive Services Task Force concludes that the current evidence is insufficient to assess the balance of benefits and harms of screening for adolescent idiopathic scoliosis in children and adolescents aged 10 to 18 (29). Since RCTs are not very feasible and unethical a multi-centre prospective study with centre-based allocation with and without screening will provide some more insights on screening for vertebral anomalies in patients with CFM. The clinical relevance of vertebral anomalies in CFM remains unclear and should be studied.

4.7.3 What are the indications and policy for treatment of vertebral anomalies in patients with craniofacial microsomia?

The literature search for this question was directed towards more specific research questions related to treatment options, symptoms, requirements, complications, and advantages and disadvantages of treatment of vertebral anomalies (Appendix 1, http://links.lww.com/SCS/B697).

\section{Review of literature}

Connell et al. studied the effect of growth-friendly surgery for early-onset scoliosis associated with CFM (18). The radiographic results and complications of this surgery were evaluated. A total of 17 patients with CFM and scoliosis were included. Ten patients 
were treated with growth-friendly surgery, three patients with fusion of vertebrae, one patient was treated with a brace, and three patients were observed. The preoperative scoliosis of the three patients who had fusion was $69^{\circ}$, which improved to $60^{\circ}$ two years postoperatively. The single patient who had a brace had a scoliosis of $11^{\circ}$ at start of the treatment. And the three patients who were observed had a scoliosis of $38^{\circ}$ (range $30^{\circ}-44^{\circ}$ ) at start, which was unchanged at two-year follow-up with a mean of $41^{\circ}$ (range $36^{\circ}$ $51^{\circ}$ ). The ten patients who received growth-friendly surgery were assessed in more detail. Nine patients had rib-based distraction (including vertical expandable prosthetic titanium rib) and one patient was treated with traditional growth-friendly rods. The mean age of the first surgery was 4.6 years (range 10 months -8 years). The mean scoliosis improved from $64^{\circ}\left(31^{\circ}-98^{\circ}\right)$ to $52^{\circ}(\mathrm{p}=0.15)$ and remained constant over two-year follow-up $\left(50^{\circ}, \mathrm{p}=0.09\right)$. The mean maximum kyphosis increased a bit after treatment from $36^{\circ}$ $\left(13^{\circ}-50^{\circ}\right)$ pre-surgery, to $38^{\circ}(\mathrm{p}=0.40)$ post-surgery and $42.4^{\circ}$ $(p=0.08)$ at two-year follow-up, although the results were not significant. At two-year follow-up post-surgery a slight, non-significant increase in high of the spine $(\mathrm{T} 1-\mathrm{S} 1)$ was seen $(23.6 \mathrm{~cm}$ to $27.3 \mathrm{~cm}, \mathrm{p}=0.06$ ); an increase of convex hemithoracic height $(7.9 \mathrm{~cm}$ to $12.8 \mathrm{~cm}, \mathrm{p}=0.05)$; a non-significant increase in concave hemithoracic height $(8.8 \mathrm{~cm}$ to $9.9 \mathrm{~cm}, \mathrm{p}=0.30)$; a non-significant increase in right hemithoracic width $(7.2 \mathrm{~cm}$ to $7.9 \mathrm{~cm}, \mathrm{p}=0.07)$; and a non-significant increase of left hemithoracic width $(7.2 \mathrm{~cm}$ to $7.9 \mathrm{~cm}, \mathrm{p}=0.43$ ). The average number of lengthening surgeries per patient was 3.0 (range 2-4). Eight of the ten patients had complication of surgery, such as infection $(n=4)$, migration $(n=3)$, pneumonia $(n=2)$, and instrumentation failure $(n=2)$. No severe complications were noted. The authors conclude that these results show that growth-friendly surgical intervention for early-onset scoliosis in CFM tends to improve scoliosis and spine height, but significantly improves convex hemithoracic height.

\section{Conclusions}

Level 3

Policy
Growth-friendly surgical intervention for
early-onset scoliosis in craniofacial
microsomia may improve the scoliosis,
spine height, and hemithoracic width and
height (studied in 17 patients).
Ref (18)

\section{Considerations}

\section{- Overall quality of evidence}

Data on treatment modalities was based on a single retrospective study, with a small patient sample, and without comparison between treatment modalities (18). No strong conclusions on the indications and policy for treatment of vertebral anomalies in CFM can be made. The quality of evidence was graded on level 3.

\section{- Balance of benefits and harms}

An important benefit of surgery is to keep patients mobile and to prevent neurological injury. Later surgery may reduce rib prominence and diminish spinal deformity. It could lead to a cosmetic improvement for some patients. Whenever surgery is indicated the benefit should clearly outweigh the risks of being conservative. Patients undergoing spinal surgery are at significant risk for the development of complications including unplanned readmission and reoperations. These outcomes are unwanted for patients and doctors and will increase costs for the healthcare system in general.
Results of surgery may vary. Mortality and complications like infection, blood loss, nerve damage, wrong screw placement, CSF leakage, and bowel and bladder problems are some of the potential risks of any type of spinal surgery, including spinal fusion for the treatment of scoliosis. Additional complications that may be associated with surgery include loss of proper spinal balance, pseudoarthrosis (no fusion), instrumentation failure and vertebral degeneration in the levels adjacent to the fused section.

Data on risks and benefits are scarce. Recently the database of the American College of Surgeons National Surgical Quality Improvement Program (ACS NSQIP), a multicentre paediatric database that aims to collect short-term data on paediatric patients, was used to determine clinical outcomes in paediatric patients undergoing spinal arthrodesis (30). The objectives were to analyse the short-term mortality rate, complications, reoperation rates and readmission rates and to identify significant patient risk factors associated with these risks. A total of 4420 paediatric patients who underwent spinal fusion were identified. Common indications for surgical intervention included acquired/idiopathic scoliosis or kyphoscoliosis (71.2\%) and genetic/syndromic scoliosis (10.7\%). They found that for paediatric patients undergoing spinal arthrodesis for all causes, there was a 3.6\% unplanned reoperation rate, a $3.96 \%$ unplanned readmission rate, and a $9.0 \%$ complication rate. Furthermore, the presence of pulmonary comorbidities and female sex represent significant risk factors for reoperation. By using risk stratification these data will allow the surgeons to identify high-risk groups to optimise shared decision making and improve outcomes.

The benefit of bracing is that it is easily applied, non-invasive and will prevent surgery in some cases. The potential harms of bracing include pressure ulcers, chest pain, physical and social limitations, anxiety, and depression. Since bracing improves outcomes in patients with idiopathic scoliosis it is likely to have the same effect in patients with CFM.

\section{- Outcome importance}

To achieve optimal outcome in care and treatment of vertebral anomalies in CFM, risk-balanced proper surgical indication and the avoidance of complications are the main goals for physicians and their patients. Not only from a surgical point of view but also from a screening and conservative point of few the goals are comparable. Surgical and non-surgical treatment, even in the best hands, has complications. Benefit to the patient should be the primary concern in the attempt to avoid negative outcomes and legal issues. Complications will have detrimental effects and will always increase the costs of treatment. Hence, a multidisciplinary approach is warranted to optimise outcomes for these patients.

\section{- Costs and resources}

The impact on costs and resources of the given recommendations will vary per member state, depending on the available care providers and facilities. Treatment of spine anomalies may reduce costs by preventing neurological deterioration. The recommendations concern the essential requirements for adequate treatment of patients with CFM and should thus be implemented. The costs of bracing are low and will prevent expensive surgery in some cases. Since the costs of spinal cord injury are high, prevention of neuronal damage is essential and will be cost effective in the long term. Surgical prevention of (neurological) deterioration including spinal cord injury will prevent additional care being necessary, especially when tertraparesis occurs (31). Costs are lowest and resources are most efficiently used when care for craniofacial disorders is centralised in a limited number of expert centres per member state. A general rule that can be applied is one expert centre per 10 million inhabitants. 


\section{- Inequity of the recommendation}

The goal of the European Reference Networks (ERNs) is to eliminate inequality within Europe with regard to care for patients with rare diseases. At present, not every member state offers an expert centre for CFM, or the level of provided care does not (yet) meet all the requirements that are outlined in this guideline. By defining the baseline of required care for CFM, this guideline will help these member states to reach the appropriate level. The ERN on craniofacial anomalies and ENT disorders (ERN-CRANIO) can guide a patient in Europe to the available centres of expertise (www.ern-cranio.eu) and can support care providers with diagnosis and treatment advice.

\section{- Feasibility of the recommendation}

Recommendations refer to the general requirements for delivering optimal healthcare and are discussed with members from participating European countries. Quality of care was paramount in the discussions. Centralisation is proposed as one of the core values. However, in some countries the national organisation of healthcare might impede centralisation. National implementation of the ERNs that fits the situation of each country is necessary. For the member states with the lowest number of inhabitants, the establishment of a craniofacial centre might not be feasible, and collaboration with a craniofacial centre in the surrounding countries can be an option.

\section{- Acceptability of the recommendation}

It is expected that all stakeholders want to apply and will be applying the recommendations, because they are employed in ERNacknowledged institutions. National implementation plans are necessary to ensure that recommendations fit the situation in each country. In addition, not all countries participating in the ERNCRANIO are represented in the guideline development group, and new members will join within the coming years. For these countries, acceptance and implementation of ERN guidelines such as this guideline on CFM is obligatory.

\section{Rationale of the recommendations}

In the most recent recommendation Statement of the USPSTF (29) there is evidence from five studies that treatment with bracing may decrease curvature progression in adolescents with mild or moderate curvature severity (an intermediate outcome). However, no adequate evidence on the association between reduction in spinal curvature in adolescence and long-term health outcomes in adulthood was found. We may extrapolate these findings to scoliosis in patients with CFM.

If the spine is unstable, surgical fusion and/or bracing may be necessary to obtain spinal stability and to prevent secondary injury of the spinal structures, including spinal cord. When conservative treatment fails, surgical intervention is usually necessary. When surgery is considered, adequate radiological imaging including MRI, CT and CT-Angiography is warranted. Surgery is less feasible in children especially at a younger age and smaller size. Awareness of age-related anatomy is indispensable in the surgical treatment of these patients. A multidisciplinary approach and shared decisionmaking should be part of treatment strategies. The choice for a specific surgical technique should be based on its benefits and harms and the surgeon's experience. Every surgical treatment technique has its own harms and benefits which are specific to the individual patient and the level of instability. For example, when cervical stabilisation is considered, detailed information about the vertebral arteries is warranted whereas for scoliosis information about tethering of the spinal cord is more important. The important objective of surgery for vertebral anomalies including scoliosis in patients with CFM is to prevent neurological damage and progression of spinal deformity and to maintain spine, chest wall, and lung development. Intraoperative neuromonitoring is considered indispensable for these types of surgery. Evidence on when, what and how surgery should be performed is often lacking. One of the evidence-based techniques available is the growth-friendly surgical intervention for early-onset scoliosis in patients with CFM. This technique improves convex hemithoracic height significantly and has a tendency to improve scoliosis (18).

\section{Recommendations}

- Surgical fusion and/or bracing in patients with vertebral anomalies may be necessary to obtain spinal stability and to prevent secondary injury of the spinal structures.

- A multidisciplinary approach in treatment and timing is warranted to optimise outcomes for these patients.

\section{Research gap}

Low-quality data make the use of evidence-based medicine in decisions on surgery for vertebral anomalies in CFM difficult. Data on the natural history, imaging, treatment, and outcomes of CFM patients with vertebral paediatric spondylolysis are scarce and treatment is mostly based on expert opinions. In most surgical cases the technique used is mainly dependent on the surgeon's preference rather than based on evidence. Large registries will provide more insight into the best treatment strategies and could be of help with making evidence-based medical decisions.

\section{References}

1. Shands AR, Jr., Eisberg HB. The incidence of scoliosis in the state of Delaware; a study of 50,000 minifilms of the chest made during a survey for tuberculosis. J Bone Joint Surg Am. 1955;37-A(6):1243-9.

2. Alexander PG, Tuan RS. Role of environmental factors in axial skeletal dysmorphogenesis. Birth Defects Res C Embryo Today. 2010;90(2):118-32.

3. Anderson PJ, David DJ. Spinal anomalies in Goldenhar syndrome. Cleft Palate Craniofac J. 2005;42(5):477-80.

4. Barisic I, Odak L, Loane M, Garne E, Wellesley D, Calzolari E, et al. Prevalence, prenatal diagnosis and clinical features of oculo-auriculo-vertebral spectrum: a registry-based study in Europe. Eur J Hum Genet. 2014;22(8):1026-33.

5. Avon SW, Shively JL. Orthopaedic manifestations of Goldenhar syndrome. J Pediatr Orthop. 1988;8(6):683-6.

6. Chan G, Dormans JP. Update on congenital spinal deformities: preoperative evaluation. Spine (Phila Pa 1976). 2009;34(17): 1766-74.

7. Wills BP, Dormans JP. Nontraumatic upper cervical spine instability in children. J Am Acad Orthop Surg. 2006;14(4): 233-45.

8. Tsou PM, Yau A, Hodgson AR. Embryogenesis and prenatal development of congenital vertebral anomalies and their classification. Clin Orthop Relat Res. 1980(152):211-31.

9. Morin B, Poitras B, Duhaime M, Rivard CH, Marton D. Congenital kyphosis by segmentation defect: etiologic and pathogenic studies. J Pediatr Orthop. 1985;5(3):309-14.

10. David L. Skaggs TG, Ron El-Hawary, John Emans, Michael Mendelow, John Smith,. Early Onset Scoliosis Consensus Statement, SRS Growing Spine Committee, 2015,. Spine Deformity,. 2015;3(2):107.

11. Yang S, Andras LM, Redding GJ, Skaggs DL. Early-Onset Scoliosis: A Review of History, Current Treatment, and Future Directions. Pediatrics. 2016;137(1). 
12. Caron C, Pluijmers BI, Wolvius EB, Looman CWN, Bulstrode $\mathrm{N}$, Evans RD, et al. Craniofacial and extracraniofacial anomalies in craniofacial microsomia: a multicenter study of 755 patients'. J Craniomaxillofac Surg. 2017;45(8): 1302-10.

13. Renkema RW, Caron C, Wolvius EB, Rooijers W, Schipper JAM, Dunaway DJ, et al. Vertebral anomalies in craniofacial microsomia: a retrospective analysis of 991 patients. Int J Oral Maxillofac Surg. 2018;47(11):1365-72.

14. Cohen N, Cohen E, Gaiero A, Zecca S, Fichera G, Baldi F, et al. Maxillofacial features and systemic malformations in expanded spectrum Hemifacial Microsomia. Am J Med Genet A. 2017;173(5):1208-18.

15. Renkema RW, Caron C, Mathijssen IMJ, Wolvius EB, Dunaway DJ, Forrest CR, et al. Vertebral anomalies in craniofacial microsomia: a systematic review. Int J Oral Maxillofac Surg. 2017;46(10):1319-29.

16. Pegler JR, Soares DC, Quaio CR, Fernandes N, Oliveira LA, Honjo RS, et al. Clinical description of 41 Brazilian patients with oculo-auriculo-vertebral dysplasia. Rev Assoc Med Bras (1992). 2016;62(3):202-6.

17. Davide B, Renzo M, Sara G, Elisa L, Rodica M, Irene T, et al. Oculo-auriculo-vertebral spectrum: going beyond the first and second pharyngeal arch involvement. Neuroradiology. 2017;59(3):305-16.

18. Connell B, Oore JJ, Pahys JM, Thompson GH, St Hilaire T, Flynn T, et al. Growth-Friendly Surgery Is Effective at Treating Early-Onset Scoliosis Associated With Goldenhar Syndrome. Spine Deform. 2018;6(3):327-33.

19. Beleza-Meireles A, Hart R, Clayton-Smith J, Oliveira R, Reis $\mathrm{CF}$, Venancio $\mathrm{M}$, et al. Oculo-auriculo-vertebral spectrum: clinical and molecular analysis of 51 patients. Eur J Med Genet. 2015;58(9):455-65.

20. Rollnick BR, Kaye CI, Nagatoshi K, Hauck W, Martin AO. Oculoauriculovertebral dysplasia and variants: phenotypic characteristics of 294 patients. Am J Med Genet. 1987;26(2): 361-75.

21. Cohen MS, Samango-Sprouse CA, Stern HJ, Custer DA, Vaught DR, Saal HM, et al. Neurodevelopmental profile of infants and toddlers with oculo-auriculo-vertebral spectrum and the correlation of prognosis with physical findings. Am J Med Genet. 1995;60(6):535-40.

22. Horgan JE, Padwa BL, LaBrie RA, Mulliken JB. OMENSPlus: analysis of craniofacial and extracraniofacial anomalies in hemifacial microsomia. Cleft Palate Craniofac J. 1995;32(5):405-12.

23. Dunn J, Henrikson NB, Morrison CC, Blasi PR, Nguyen M, Lin JS. Screening for Adolescent Idiopathic Scoliosis: Evidence Report and Systematic Review for the US Preventive Services Task Force. JAMA. 2018;319(2):173-87.

24. Frush DP, Donnelly LF, Rosen NS. Computed tomography and radiation risks: what pediatric health care providers should know. Pediatrics. 2003;112(4):951-7.

25. Adelgais KM, Grossman DC, Langer SG, Mann FA. Use of helical computed tomography for imaging the pediatric cervical spine. Acad Emerg Med. 2004;11(3):228-36.

26. Brenner D, Elliston C, Hall E, Berdon W. Estimated risks of radiation-induced fatal cancer from pediatric CT. AJR Am J Roentgenol. 2001;176(2):289-96.

27. Akbarnia BA. Pediatric spine fractures. Orthop Clin North Am. 1999;30(3):521-36, x.

28. Trenga AP, Singla A, Feger MA, Abel MF. Patterns of congenital bony spinal deformity and associated neural anomalies on X-ray and magnetic resonance imaging. J Child Orthop. 2016;10(4):343-52.
29. Force USPST, Grossman DC, Curry SJ, Owens DK, Barry MJ, Davidson KW, et al. Screening for Adolescent Idiopathic Scoliosis: US Preventive Services Task Force Recommendation Statement. JAMA. 2018;319(2):165-72.

30. Abu-Bonsrah N, Goodwin CR, Ortega G, Abdullah F, Cornwell E, De la Garza-Ramos R, et al. Risk factors associated with short-term complications and mortality after pediatric spinal arthrodesis. Neurosurg Focus. 2017;43(4):E7.

31. McDaid D, Park AL, Gall A, Purcell M, Bacon M. Understanding and modelling the economic impact of spinal cord injuries in the United Kingdom. Spinal Cord. 2019.

\section{CHAPTER 4. SCREENING, MONITORING AND INDICATION FOR TREATMENT}

\subsection{Psychosocial difficulties in craniofacial microsomia \\ Introduction}

Atypical facial appearance can lead to poor social acceptance by others and cause psychosocial difficulties $(1,2)$. Social comparison begins in primary school and increases at least into adolescence, with comparisons to peers and those portrayed in the media, as well as the influence of comments from peers and parents, transforming beliefs and stereotypes about attractiveness into self-evaluation concerning appearance (1). Patients with an unusual facial appearance or craniofacial syndromes can have difficulties with psychological adjustment and experience teasing. Various forms of teasing or bullying have been described, such as staring, whispering, making inappropriate comments, aggression, emotional distress, and/or social withdrawal (3-5). Teasing was found to be associated with a higher risk for depression, social difficulties, and aggression in patients with microtia $(6,7)$. Teasing is common in these patients: $30 \%$ to $100 \%$ of patients with microtia reported being teased, starting in early childhood $(8,9)$.

Although patients with craniofacial syndromes are at increased risk for psychosocial problems, parents/caregivers of these patients also experience difficulties. Raising a child with a craniofacial anomaly is demanding and leads to stress in parents due to various reasons, such as multiple medical appointments, functional problems, teasing, psychosocial problems, or learning difficulties $(10-14)$.

Intellectual abilities, language or speech development, or other neurodevelopmental processes are important for adequate psychosocial functioning. Although some craniofacial syndromes are associated with neurodevelopmental delay, this is less clear for patients with craniofacial microsomia (15).

To obtain more knowledge regarding psychosocial difficulties in craniofacial microsomia (CFM) and offer potential recommendations on screening, monitoring, and treatment, the following questions were posed:

4.8.1 What is the type, prevalence and severity of psychosocial difficulties in craniofacial microsomia?

4.8.2 What is the policy for screening and monitoring of psychosocial difficulties in patients with craniofacial microsomia?

4.8.3 What are the indications and policy for treatment of psychosocial difficulties in patients with craniofacial microsomia?

Some characteristics of patients with CFM may increase the risk for psychosocial difficulties. Difficulties in facial expressiveness or eye contact may be caused by facial nerve deficits in some patients 
with CFM (16). Difficulties with hearing, speech or vision have been associated with psychiatric disorders, behavioural problems, and/or social difficulties (17-20). Additionally, the increased risk for teasing or potential neurodevelopmental delays may also cause a higher risk for psychosocial difficulties in patients with CFM.

Literature search

A systematic search of literature was performed to identify all available literature on craniofacial microsomia and synonyms. The search was conducted in Embase, Pubmed/Medline Ovid. The full search strategy is reported in the supplementary material.

Inclusion and exclusion criteria:

Type of studies - Original articles

- Systematic review of sufficient quality:

- The question in the systematic review matches the question of the guideline.

- The search of the systematic review was conducted in at least two relevant databases, such as the Cochrane Library, Medline/Pubmed.

- The full search strategy was reported.

- No relevant items were missing in the search strategy.

Type of patients - Patients with craniofacial microsomia

\begin{tabular}{ll}
\hline Subject & $\begin{array}{l}\text { - Psychosocial functioning/difficulties, } \\
\text { neurodevelopment }\end{array}$ \\
\hline Exclusion criteria & - Original studies with $<10$ included patients \\
& - Articles published before 1980 \\
& - Case reports \\
& - Expert opinion \\
- Letters & - Editorials \\
- Narrative reviews
\end{tabular}

The inclusion and exclusion criteria are reported in Chapter 2 Methodology. Literature was screened on title and abstract. A total of fifteen articles were included in this chapter: eight articles on psychosocial functioning in CFM and seven articles on neurodevelopment of patients with CFM. Of the eight articles on psychosocial difficulties, three were case-control studies: Dufton et al. (21), Khetani et al. (22), and Wallace et al. (23). The other five studies were cross-sectional studies: Johns et al. (24), Luquetti et al. (25), Ongkosuwito et al. (26), Padwa et al. (27), and Volpicelli et al. (28). Seven studies on neurodevelopment of patients with CFM were included. Three case-control studies: Collett et al. (29), Speltz et al. 2017 (30), and Speltz et al. 2018 (31). Of the other four studies, three were cross-sectional studies by Cohen et al. 1995 (32), Cohen et al. 2017 (33), and Johansson et al. (34), and one study was a retrospective cohort study by Strömland et al. (35).

4.8.1 What are the type, prevalence and severity of psychosocial difficulties in craniofacial microsomia?

The literature search for this question was directed towards more specific questions related to the type, prevalence and severity of psychosocial difficulties in CFM (|Appendix 1, http://links.lww.$\mathrm{com} / \mathrm{SCS} / \mathrm{B} 697)$. No literature was available on the severity of psychosocial difficulties, therefore no conclusions were written. Since this question does not relate to interventions or diagnostics, no considerations, rationale or recommendations are provided.

\section{Review of literature Psychosocial}

Dufton et al. (21) aimed to determine the risk for psychosocial difficulties in children with CFM compared to controls. A total of 136 children with CFM and 568 healthy controls, age five to ten, were assessed for social competence, and behavioural and emotional difficulties using questionnaires that were completed by the parents and teachers of the children. Parents scored patients with CFM higher on the social difficulties scale compared to controls, but the effect size was small $(E S=0.30, p=0.002)$. Teachers rated patients with CFM to have slightly more behavioural problems, such as feeling depressed/withdrawn $(E S=0.27)$, social difficulties $(\mathrm{ES}=0.55)$, cognitive difficulties $(\mathrm{ES}=0.29)$, attention problems $(\mathrm{ES}=0.24)$, and aggressive behaviour $(\mathrm{ES}=0.21)$. Teachers rated patients with CFM as having poorer academic skills $(E S=0.21)$, poorer communication skills $(\mathrm{ES}=0.19)$, and as having lower overall social competence ( $\mathrm{ES}=0.24)$, compared to controls. These differences on social competence were largest for female patients $(E S=0.35)$ and for cases of young mothers $(E S=0.70)$. Teachers ranked patients with CFM as having slightly lower peer acceptance in the classroom (difference of 10\%), compared to controls. Especially patients with oral clefts and eye anomalies in combination with jaw and/or ear anomalies were scored as having lower peer acceptance compared to controls. Although teachers rated children with CFM to have more behavioural and social problems than controls, parents of patients and controls did not report a difference regarding these topics. This variance may be caused by a difference in the social context in which the children are observed. However, reporting bias may also have led to this difference, where parents might minimise problems of their own child or teachers might overestimate problems due to the different facial appearance of patients with CFM.

In 2018, Johns et al. (24) asked patients with CFM and parents of patients to describe experiences in their daily life and give suggestions for improving healthcare. A total of 42 caregivers and 9 adult patients with CFM were included. Caregivers $(62 \%)$ and patients (57\%) noted social stigma as a primary concern. In the community setting, $29 \%$ of the patients sometimes hid their diagnosis and $17 \%$ of the caregivers experienced explaining the diagnosis of CFM to others as difficult. Caregivers found support through support websites $(71 \%)$, their partner/spouse $(62 \%)$, family $(57 \%)$, medical professionals $(48 \%)$, friends $(41 \%)$, and communicating with fellow patients $(41 \%)$. Around half of the adult patients $(56 \%)$ and $27 \%$ of the caregivers reported active teasing. Passive social exclusion was also frequently reported $33 \%$ of the patients and $12 \%$ of the caregivers). A fifth $(22 \%)$ of the patients tried to hide their diagnosis, and $11 \%$ experienced issues with social comparison and difficulties in having a romantic relationship.

Khetani et al. (22) aimed to study the health-related quality of life in children with CFM by assessing parents of patients and patients during the elementary school years. More controls $(n=568)$ than patients with CFM $(n=136)$ were included. Both patients and controls had a mean age close to seven years $( \pm$ one year). Parents scored children with CFM lower on measures of physical, social, and school functioning, compared to controls. Although this was a significant difference, the effect sizes were small (ES: 0.26-0.34). On the child-reported assessment, no differences in in physical, social, or emotional functioning between children with CFM and controls were found.

Luquetti et al. (25) studied the diagnostic, treatment-related, and early psychosocial experiences of 9 adult patients with CFM and 42 caregivers of children with CFM. Caregivers experienced feelings of concern/anxiety (79\%), surprise/shock (64\%), sadness $(64 \%)$, guilt $(55 \%)$, and confusion $(31 \%)$ in response to hearing the diagnosis of CFM. Teasing was common for patients with CFM: all adults but one were teased during childhood and caregivers reported that $43 \%$ of the children aged four and older were teased. Most teasing occurred in childhood (age five to ten) with a peak of teasing at a mean age of nine years (SD 2.5). Name calling was the most frequent form of teasing (caregivers $56 \%$, patients $83 \%$ ), 
although questions about their appearance or nonverbal negative reactions of mimicking or running away occurred too (caregivers $22 \%$, patients $17 \%)$. In response to teasing most caregivers $(45 \%)$ and patients $(67 \%)$ said to ignore the teasing. Being stared at was also frequently noted by patients. Although some patients did not care $(13 \%)$, others described feeling shy or having negative emotional responses (caregivers 13\%, patients 38\%). Most patients had adaptive coping responses to staring (caregivers $26 \%$, patients $38 \%$ ), others ignored staring (caregivers $6 \%$, patients $13 \%$ ), or felt reminded about their diagnosis of CFM (caregivers 3\%, patients $25 \%)$.

Stress in parents of children with CFM and their coping styles were studied by Ongkosuwito et al. (26). Questionnaires on parental stress and on cognitive emotion-regulation were completed by 31 parents of patients with CFM. Learning difficulties and psychosocial difficulties were associated with an increase in parental stress $(\mathrm{r}=0.71, \mathrm{r}=0.63$, both $\mathrm{p}=<.001)$. No relation between hearing difficulties $(r=0.32, p=0.08)$, articulation difficulties $(r=0.34$, $\mathrm{p}=0.06)$, or the facial appearance of the child $(\mathrm{r}=0.07, \mathrm{p}=0.72)$ and parental stress was found. The coping strategies that had a positive correlation with parental stress were acceptance and positive reappraisal $(\mathrm{r}=0.50, \mathrm{p}=0.01$ and $\mathrm{r}=0.54, \mathrm{p}=0.002)$. Multivariate regression analysis showed that learning difficulties and the use of acceptance as a coping strategy explained a large proportion of parental stress. Interventions for parents of children that address learning difficulties and coping strategies could potentially be beneficial to lower parental stress.

Padwa et al. (27) studied psychosocial adjustment in children with craniofacial anomalies to examine the relationships between symmetry, functional impairments, and social adaptation. Of the 30 patients included, 11 had CFM. Most patients (80\%) with CFM were able to socialise in a positive way by joining the group, which was higher when compared to other craniofacial anomalies $(52 \%$ $57 \%$ ). Low self-esteem and negative self-concept were reported by $35-40 \%$ of all children; no subgroup data of patients with CFM was reported. Patients with symmetrical craniofacial anomalies had a significantly higher prevalence of low mood. Patients with symmetrical and non-CFM craniofacial anomalies had lower social competences scores. This suggests a higher prevalence of low mood, behavioural problems, and socialising problems were present in this group when compared to patients with asymmetrical craniofacial disorders, and to patients with CFM. The authors debate whether children with facial asymmetry may be viewed as having a normal face with imperfections, while patients with a symmetrical craniofacial anomaly could be seen as having a severe medical condition or syndrome. Patients with an asymmetric deformity may be more acceptable to themselves and experience fewer psychosocial difficulties. The study concludes that early surgery in patients with symmetrical craniofacial deformities may help to prevent psychosocial difficulties. However, no evidence supporting this theory is provided. Additionally, it is important to note the small sample size of patients with CFM in this study.

The timing of paediatric craniofacial surgery and its effect on psychosocial development was further examined by Volpicelli et al. (28). They aimed to study differences in psychosocial outlook in children with craniofacial anomalies under active treatment. A total of 99 patients with a craniofacial disorder such as cleft lip/palate or craniofacial syndrome, age 8-17 years, were included. Of these 99 patients, 22 patients had CFM (21.6\%). Younger patients (age 810 ) were found to have significantly higher anxiety, depression, and anger scores, and lower peer relationship scores, compared to older patients (age 11-13 and/or age 14-17) and to the U.S. average scores. The authors argue that more prevention and intervention methods may be needed for families and schools to help younger patients (age 8-10).
Wallace et al. (23) studied psychosocial adjustment in adolescents with CFM and compared this with controls. A total of 142 patients with CFM and 316 healthy controls were included, with a mean age of 13.4 years (SD 1.4 years, range $10-17$ years). Both patients with CFM and controls reported similar levels of behavioural problems. However, lower social and school functioning scores were reported by patients with CFM and parents of patients due to various reasons such as teasing, not being able to participate with other teens, or missing school due to hospital visits. Although patients with CFM had slightly more difficulties in social functioning compared to controls, fewer problems with externalising behaviour were reported compared to controls. The authors discuss that the higher frequency of social difficulties in patients with CFM may be the result of teasing, rejection, or stigmatisation, instead of a personal trait. No strong evidence for differences in behavioural adaptation or socialisation based on the severity of the facial malformations was found.

\section{Neurodevelopment}

Speltz et al. (2017) (30) studied the intelligence and academic achievements of adolescents with CFM. A total of 142 patients with CFM and 316 controls were included (mean age 13 years, range $11-$ 17 years). Patients with CFM scored significantly lower on IQ and academic achievements ( $\mathrm{ES}=-0.01$ to -0.3 , p-values range 0.01 to 0.92 ). It is important to note that this effect size is relatively small. Learning difficulties occurred in $25 \%$ of the controls and in $38 \%$ of the patients with CFM (adjusted OR $=1.5$, 95\% CI $0.9-$ 2.4). Patients with mandibular hypoplasia and microtia had significantly lower IQ and achievement scores as did patients with isolated microtia, when compared to controls. Although $70 \%$ of the patients with CFM had hearing difficulties compared to $1 \%$ of the controls, an effect of hearing difficulties on IQ and achievements scores was not found. The authors conclude that these findings should be viewed 'primarily as hypothesis-generating, rather than as firm conclusions'. According to the authors, developmental surveillance of children and adolescents with CFM should be undertaken.

In another study by Speltz et al. (2018) (31) the neurodevelopmental profile of infants with CFM was assessed and compared with controls. A total of 108 patients with CFM and 84 controls, all age $12-24$ months, were included. Developmental delay was found in $21 \%$ of the patients with CFM and in $16 \%$ of the controls. However, no differences in terms of developmental delay were present after adjustment for demographic factors $(\mathrm{OR}=0.68$, 95\% CI $0.29-$ 1.61). No differences in development were found based on the CFM phenotype. The authors conclude that no evidence for neurodevelopmental differences between infants with CFM (age one to two) and controls could be found.

Collett et al. (29) aimed to determine the risk for neurodevelopmental delay in 136 children with CFM compared to 568 healthy controls. Lower verbal, nonverbal, and academic skills were seen in patients with CFM compared to controls. Patients with CFM had a significantly higher risk for receptive language delays and visuomotor skills delay compared to controls. Worse outcomes on the neurodevelopmental profile were seen in patients born to mothers who were 25 or younger at time of birth. The authors emphasise the need to carry out routine neurodevelopmental screening for patients with CFM.

A smaller, cross-sectional study by Cohen et al. (1995) (32) included 24 patients with CFM to examine their neurodevelopmental profile. No significant difference in mean IQ compared to the general population was found. A delay was found in gross $(38 \%)$ and fine $(20 \%)$ motor development and in receptive $(32 \%)$ and expressive (37\%) language development compared to the general population. Receptive and expressive language development were significantly lower in patients with bilateral CFM compared to 
unilateral CFM. In a larger cross-sectional study by Cohen et al. (2017) (33) on facial and systemic malformations in a cohort of 89 patients with CFM, $5 \%(n=4)$ were found to have an intellectual disability. No further information on developmental delay was provided in this study.

Johansson et al. (34) aimed to study autism spectrum disorder in 20 patients with CFM (mean age 8 years, range 8 months -17 years). Eleven patients $(55 \%)$ were found to have normal intelligence and nine patients $(45 \%)$ had learning disabilities, which were mild in four patients $(20 \%)$, severe in three patients $(15 \%)$, and profound in two patients (10\%). Autism spectrum disorder was diagnosed in two patients $(10 \%)$, autism-like condition in one patient $(5 \%)$, and autistic traits in five patients $(25 \%)$. Behavioural disturbances, such as aggressive behaviour or hyperactivity, were solely seen in patients with autism spectrum disorders.

Strömland et al. (35) also studied autism spectrum disorder in CFM. One of their eighteen included patients with CFM had autism and two met the criteria for childhood autism/autistic-like disorder. Six other patients had learning disabilities (30\%): three had severe $(17 \%)$ and three had mild (17\%) learning disabilities.

\section{Conclusions}

Level 3 Type
Patients and/or parents of patients with craniofacial
microsomia have a higher risk of experiencing social
stigma, low self-esteem and active or passive bullying,
especially in childhood.
Parental stress in parents of patients with craniofacial
microsomia is linked to their child's learning difficulties,
and parental acceptance. The facial appearance of the
child does not appear to be an important influential factor
in parental stress.
Ref $(24-27)$

\section{Level 3 Risk factors}

Patients with symmetrical craniofacial anomalies may experience more behavioural problems, depressed feelings, and socializing problems compared to patients with an asymmetrical craniofacial anomaly.

Younger children (age 8-10) with a craniofacial anomaly may experience more problems with anxiety, low mood, anger, and peer relationships compared to older children (age 11-17).

$\operatorname{Ref}(27,28)$

\section{Level 3 Type - Neurodevelopmental}

Patients with craniofacial microsomia may have a slightly lower IQ score and more learning difficulties compared to healthy controls.

The neurodevelopmental profile of infants with craniofacial microsomia (age 1-2 years) is not different from healthy controls.

Patients with craniofacial microsomia might have a higher risk for language- and motor skills delays compared to healthy controls.

Ref (29-35)

4.8.2 What is the policy for the screening and monitoring of psychosocial difficulties in patients with craniofacial microsomia?

The literature search for this question was directed towards more specific questions related to the consequences and impact of screening and monitoring, the available screening tests and procedures for monitoring, and responsibilities for screening and monitoring (Appendix 1, http://links.lww.com/SCS/B697). Unfortunately, no literature is available on the policy for screening and/or monitoring of psychosocial difficulties in patients with CFM. Therefore no conclusions were written. Considerations for this question were only based on expert opinion.

\section{Considerations}

\section{- Overall quality of evidence}

Because of the lack of evidence, the recommendations on screening and monitoring of psychosocial difficulties in CFM are based on expert opinion with a low quality of evidence.

\section{- Balance of benefits and harms}

Having access to a psychologist and a team specialising in CFM will have obvious biopsychosocial benefits for patients and their families including providing a high standard of care, improving patient quality of life and potentially reducing hospital stays and communications.

\section{- Outcome importance}

The main reason to perform screening is to help to reduce emotional distress experienced by patients and their families, promote information sharing and a holistic approach, and pre-empt psychological difficulties developing as a result of the patient's physical health condition.

\section{- Costs and resources}

The impact on costs and resources of the given recommendations will vary per member state, depending on the available care providers and facilities. The recommendations concern the essential requirements for adequate treatment of patients with CFM and should thus be implemented. Costs are lowest and resources are most efficiently used when care for craniofacial disorders is centralised in a limited number of expert centres per member state. A general rule that can be applied is one expert centre per 10 million inhabitants.

\section{- Inequity of the recommendation}

The goal of the European Reference Networks (ERNs) is to eliminate inequality within Europe with regard to care for patients with rare diseases. At present, not every member state offers an expert centre for CFM, or the level of provided care does not (yet) meet all the requirements that are outlined in this guideline. By defining the baseline of required care for CFM, this guideline will help these member states to reach the appropriate level. The ERN on craniofacial anomalies and ENT disorders (ERN-CRANIO) can guide a patient in Europe to the available centres of expertise (www.ern-cranio.eu) and can support care providers with diagnosis and treatment advice.

\section{- Feasibility of the recommendation}

Recommendations refer to the general requirements for delivering optimal healthcare and are discussed with members from participating European countries. Quality of care was paramount in the discussions. Centralisation is proposed as one of the core values. However, in some countries the national organisation of healthcare might impede centralisation. National implementation of the ERNs that fits the situation of each country is necessary. For the member states with the lowest number of inhabitants, the establishment of a craniofacial centre might not be feasible, and collaboration with a craniofacial centre in the surrounding countries can be an option. 


\section{- Acceptability of the recommendation}

It is expected that all stakeholders want to apply and will be applying the recommendations, because they are employed in ERNacknowledged institutions. National implementation plans are necessary to ensure that recommendations fit the situation in each country. In addition, not all countries participating in the ERNCRANIO are represented in the guideline development group, and new members will join within the coming years. For these countries, acceptance and implementation of ERN guidelines such as this guideline on CFM is obligatory.

\section{Rationale of the recommendations}

The rationale for having psychological input in a specialist team for CFM is to pre-empt any psychological issues experienced by the child and family that could have an impact on the patient journey, and to ensure that the team approach is holistic (36). It may involve mediating on behalf of the family with medical teams or schools, or between child and parent if there is a difference in opinion regarding intervention. The overall aim is to reduce distress and to enhance and promote psychological wellbeing. The amount of distress does not always seem to be proportionate to objective appearance, as it is a psychological process that determines response to differences in appearance. Regular review by the specialist team offers the patient and family holistic and up-to-date help. Regular contact fosters good relationships between professionals and the family and allows the team to discuss intervention in an age-appropriate manner. It also allows the family to ask about new developments in the field and if necessary, to discuss psychosocial, educational and hearing issues which may arise. Being provided with this information and support promotes parental adjustment and acceptance and enables them to communicate positive themes of normalisation and reassurance, which children mirror in their own responses and adjustment (37). The ICHOM Standard Set for Craniofacial Microsomia advises to see patients and their family at age 2, 5,8 , and 22 to measure psychosocial wellbeing and quality of life using the ClefQ, YP-CORE, HADS, and CFEQ. It is advised to use the Distress Thermometer for Parents to measure family stress.

It is good practise to advise families of active voluntary sector support groups and relevant charities. Support groups and charities can help children and their families deal with difference, and promote confidence, resilience and positive self-image. Healthcare providers play a key role in family adjustment by clarifying misinformation, providing education regarding treatment options, modelling acceptance, screening for psychosocial concerns, providing resources and connecting families to support networks. With regards to visible differences, patient coping and level of concern is related to personality, family responses, school support and developmental stage. Predictors of good psychosocial outcomes are social and family support, perceptions of severity and social interaction skills, and clinicians (with regular contact and working with the system) can to have a positive influence on these factors.

\section{Recommendations}

- All craniofacial microsomia patients should have access to a clinical psychology service with appropriate professional expertise and knowledge of craniofacial microsomia.

- Time points for reviews and screening should observe key life transitions such as birth, starting school, transition to secondary school, etc.
- To measure psychosocial wellbeing and family stress, validated self-reported psychological outcome measures should be obtained from to all craniofacial microsomia patients as a matter of routine to screen for the presence of behavioural, emotional, social and/or learning difficulties. This includes the CleftQ, CFEQ, YP-CORE, HADS and Distress Thermometer for Parents and should be performed at age 2, 5, 8 and 22. Elevated scores should alert clinicians to the potential need for further assessment or support. Standardised measures should assess levels of emotional distress as well as evaluate difficulties related to visible differences.

\section{Research gap}

It is recognised that there is a lack of quantitative and qualitative research looking at the psychological impact of CFM, and a need for longitudinal studies to further inform psychological assessments and interventions. Use of measures pre- and post-operatively provides valuable data for audit and research as well as quickly identifying any psychological difficulties that can potentially be addressed, and themes and issues identified in clinics directly from patients and their families could be assessed using qualitative methods.

4.8.3 What are the indications and policies for the treatment of psychosocial difficulties in patients with craniofacial microsomia?

The literature search for this question was directed towards more specific research questions related to treatment options, symptoms, requirements, complications, and advantages and disadvantages of treatment of psychosocial difficulties (Appendix 1, http:// links.lww.com/SCS/B697). Unfortunately, no literature is available on the policy for treatment of psychosocial difficulties in patients with CFM. Therefore no conclusions were written. Considerations for this question were only based on expert opinion.

\section{Considerations}

\section{- Overall quality of evidence}

Because of the lack of evidence, the recommendations on treatment of psychosocial difficulties in CFM are based on expert opinion with a low quality of evidence.

\section{- Balance of benefits and harms}

Psychological therapy can be time consuming, but interventions are individualised and planned collaboratively with the patient and their family, which increases acceptability and adherence. Managing the patient 'system' can be heavy work for clinicians, requiring communications and meetings with family members, school, other medical teams etc.

\section{- Outcome importance}

Psychosocial support and psychological intervention are designed to promote quality of life, which is important to patients, family, and clinicians. Early psychological intervention would reduce the risk of patients developing long-term mental health difficulties and their associated sequela, thus reducing the strain on healthcare resources in future years.

\section{- Costs and resources}

The impact on costs and resources of the given recommendations will vary per member state, depending on the available care 
providers and facilities. The recommendations concern the essential requirements for adequate treatment of patients with CFM and should thus be implemented. Costs are lowest and resources are most efficiently used when care for craniofacial disorders is centralised in a limited number of expert centres per member state. A general rule that can be applied is one expert centre per 10 million inhabitants.

\section{- Inequity of the recommendation}

The goal of the European Reference Networks (ERNs) is to eliminate inequality within Europe with regard to care for patients with rare diseases. At present, not every member state offers an expert centre for CFM, or the level of provided care does not (yet) meet all the requirements that are outlined in this guideline. By defining the baseline of required care for CFM, this guideline will help these member states to reach the appropriate level. The ERN on craniofacial anomalies and ENT disorders (ERN-CRANIO) can guide a patient in Europe to the available centres of expertise (www.ern-cranio.eu) and can support care providers with diagnosis and treatment advice.

\section{- Feasibility of the recommendation}

Recommendations refer to the general requirements for delivering optimal healthcare and are discussed with members from participating European countries. Quality of care was paramount in the discussions. Centralisation is proposed as one of the core values. However, in some countries the national organisation of healthcare might impede centralisation. National implementation of the ERNs that fits the situation of each country is necessary. For the member states with the lowest number of inhabitants, the establishment of a craniofacial centre might not be feasible, and collaboration with a craniofacial centre in the surrounding countries can be an option.

\section{- Acceptability of the recommendation}

It is expected that all stakeholders want to apply and will be applying the recommendations, because they are employed in ERNacknowledged institutions. National implementation plans are necessary to ensure that recommendations fit the situation in each country. In addition, not all countries participating in the ERNCRANIO are represented in the guideline development group, and new members will join within the coming years. For these countries, acceptance and implementation of ERN guidelines such as this guideline on CFM is obligatory.

\section{Rationale of the recommendations}

We suggest that including a psychologist in the specialist team or having access to paediatric or adult health psychologists improves overall psychosocial outcomes for people with CFM. Interventions can be targeted to the patient, family or the wider system, to enhance psychological wellbeing, acceptance and adjustment. Having a psychologist present enables psychosocial difficulties to be identified, with the aim being early intervention. Support groups and charities play a key role in psychosocial wellbeing, helping children and their families to deal with difference and promote confidence, resilience and positive self-image.

Healthcare providers play a key role in individual coping and adjustment, for example, by promoting positive and assertive strategies when dealing with the reactions of others, focusing on personal achievements, and encouraging participation in social and physical activities, which in turn can lead to greater self-acceptance. They also play a key role in supporting parents/carers of children with CFM in terms of making decisions regarding whether or not to proceed with surgical reconstruction, which can feel like a huge responsibility for a parent/carer to have (38).

In terms of involving the child in decision making surrounding surgery, we suggest that (where possible) there is a strong argument for waiting until the child is old enough, with the necessary level of abstract conceptual thinking and emotional maturity, to enable informed decision making. A study that interviewed adolescents with CFM found that the children were glad that their parents had allowed them to be involved in the decision-making process surrounding surgery (39). They also did not feel that concern regarding current or potential bullying was a good reason to have surgery at a young age, and that promoting self-acceptance and resilience were far more important. They were asked to list positive aspects of growing up with a craniofacial condition (39). Positive changes in personality included being more open-minded, less judgemental, kinder, and more independent. Participants also expressed the belief that they are strong individuals who are able to overcome challenges. When asked what advice they would give younger children growing up with similar conditions, participants said they would advise them to be open and honest and not be ashamed of their visible differences.

If indicated, a psychologist should be involved when proceeding with surgery at preadmission stage to assess psychological readiness for surgery, including identifying any procedural anxiety that will impact on the surgery itself, and psychosocial issues that may affect treatment adherence post-operatively. Assessment of readiness for surgery should occur before or at the preadmission clinic. A semi-structured interview format should be used to establish current emotional state, family functioning, mental and physical health history, significant life events, risk factors for poor psychological adjustment post-surgery including unrealistic expectations, concerns regarding the process of hospital admission, surgery, post-operative pain etc. As well as collating feedback for the medical team, the aim should be to attempt to ascertain if surgical reconstruction will contribute to long-term psychological wellbeing. Post-operative follow-up should occur following completion of surgical reconstruction to assess emotional state, satisfaction with surgical process, and body image issues. It is important that the patient understands the nature of the healing process and its potential impact on their body image. With patients often waiting a long time before the surgery can be performed and with the cosmetic result not fully visible for a number of weeks due to bruising and swelling, expectations must be carefully managed. Whilst it can be several weeks before patients feel fully physically recovered from reconstructive surgery, it could be argued that the bigger challenge at this time will be the need to adapt to the look and feel of their new appearance. Revealing the newly reconstructed area for the first time must be done with sensitivity and in a way that allows them some control over how and when it is seen, as well as who will see it.

\section{Recommendations}

Parental adjustment and support

- Parents of newly diagnosed children with craniofacial microsomia should have access to a specialist clinical psychology service with expertise and knowledge of the condition.

- Information on support groups and organisations should be provided, both at initial contact and at regular review.

\section{Behavioural and/or learning difficulties}

- When appropriate, clinicians should liaise with local services and schools to discuss the child's support needs.

- Cognitive assessment may be offered if warranted 
Coping with visible difference

- Patients with craniofacial microsomia should have access to specialist psychological support, particularly those who are presenting with low self-esteem, depression/low mood, anxiety, appearance- or treatment-related concerns, including adjustment difficulties or trauma as a result of surgical/medical interventions.

- Clinicians with appropriate professional expertise in craniofacial microsomia should consider liaising with local schools to offer advice on how to support children who have visible differences.

- Information about support groups and organisations should be provided.

- Psychological input is required pre- and post- facial surgery to monitor expectation and acceptance.

- The psychologist is part of the coordinated care in the multidisciplinary team. See recommendations in Chapter 6.

\section{Research gap}

As described in Chapter 4.8.2.

\section{References}

1. Dion K, Berscheid, E. Physical attractiveness and peer perception among children. Sociometry. 1974;37(1):1-12.

2. Gertner BL, Rice ML, Hadley PA. Influence of communicative competence on peer preferences in a preschool classroom. J Speech Hear Res. 1994;37(4):913-23.

3. Rumsey N, Harcourt D. Body image and disfigurement: issues and interventions. Body Image. 2004;1(1):83-97.

4. Topolski TD, Edwards TC, Patrick DL. Quality of life: how do adolescents with facial differences compare with other adolescents? Cleft Palate Craniofac J. 2005;42(1):25-32.

5. Masnari O, Landolt MA, Roessler J, Weingaertner SK, Neuhaus K, Meuli M, et al. Self- and parent-perceived stigmatisation in children and adolescents with congenital or acquired facial differences. J Plast Reconstr Aesthet Surg. 2012;65(12):1664-70.

6. Jiamei D, Jiake C, Hongxing Z, Wanhou G, Yan W, Gaifen L. An investigation of psychological profiles and risk factors in congenital microtia patients. J Plast Reconstr Aesthet Surg. 2008;61 Suppl 1:S37-43.

7. Brent B. The pediatrician's role in caring for patients with congenital microtia and atresia. Pediatr Ann. 1999;28(6): 374-83.

8. Johns AL, Lucash RE, Im DD, Lewin SL. Pre and postoperative psychological functioning in younger and older children with microtia. J Plast Reconstr Aesthet Surg. 2015;68(4):492-7.

9. Johns AL, Lewin SL, Im DD. Teasing in younger and older children with microtia before and after ear reconstruction. J Plast Surg Hand Surg. 2017;51(3):205-9.

10. Speltz ML, Endriga MC, Hill S, Maris CL, Jones K, Omnell ML. Cognitive and psychomotor development of infants with orofacial clefts. J Pediatr Psychol. 2000;25(3):185-90.

11. Hunt O, Burden D, Hepper P, Stevenson M, Johnston C. Parent reports of the psychosocial functioning of children with cleft lip and/or palate. Cleft Palate Craniofac J. 2007;44(3):304-11.

12. Collett BR, Speltz ML. A developmental approach to mental health for children and adolescents with orofacial clefts. Orthod Craniofac Res. 2007;10(3):138-48.
13. Sarimski K. Children with Apert syndrome: behavioural problems and family stress. Dev Med Child Neurol. 1998;40(1):44-9.

14. Nelson P, Glenny AM, Kirk S, Caress AL. Parents' experiences of caring for a child with a cleft lip and/or palate: a review of the literature. Child Care Health Dev. 2012;38(1):6-20.

15. Mathijssen IM. Guideline for Care of Patients With the Diagnoses of Craniosynostosis: Working Group on Craniosynostosis. J Craniofac Surg. 2015;26(6):1735-807.

16. Rives Bogart K, Matsumoto D. Facial mimicry is not necessary to recognize emotion: Facial expression recognition by people with Moebius syndrome. Soc Neurosci. 2010;5(2): 241-51.

17. Brown R, Hobson RP, Lee A, Stevenson J. Are there "autisticlike" features in congenitally blind children? J Child Psychol Psychiatry. 1997;38(6):693-703.

18. Fellinger J, Holzinger D, Sattel H, Laucht M, Goldberg D. Correlates of mental health disorders among children with hearing impairments. Dev Med Child Neurol. 2009;51(8):635-41.

19. McAlpine LM, Moore, C. The development of social understanding in children with visual impairments. Journal of Visual Impairment and Blindness. 1993;89:349-58.

20. van Daal J, Verhoeven L, van Balkom H. Behaviour problems in children with language impairment. J Child Psychol Psychiatry. 2007;48(11):1139-47.

21. Dufton LM, Speltz ML, Kelly JP, Leroux B, Collett BR, Werler MM. Psychosocial outcomes in children with hemifacial microsomia. J Pediatr Psychol. 2011;36(7):794-805.

22. Khetani MA, Collett BR, Speltz ML, Werler MM. Healthrelated quality of life in children with hemifacial microsomia: parent and child perspectives. J Dev Behav Pediatr. 2013;34 (9):661-8.

23. Wallace ER, Collett BR, Heike CL, Werler MM, Speltz ML. Behavioral-Social Adjustment of Adolescents with Craniofacial Microsomia. Cleft Palate Craniofac J. 2018;55(5):664-75.

24. Johns AL, Luquetti DV, Brajcich MR, Heike CL, Stock NM. In Their Own Words: Caregiver and Patient Perspectives on Stressors, Resources, and Recommendations in Craniofacial Microsomia Care. J Craniofac Surg. 2018;29(8):2198-205.

25. Luquetti DV, Brajcich MR, Stock NM, Heike CL, Johns AL. Healthcare and psychosocial experiences of individuals with craniofacial microsomia: Patient and caregivers perspectives. Int J Pediatr Otorhinolaryngol. 2018;107:164-75.

26. Ongkosuwito E, van der Vlies L, Kraaij V, Garnefski N, van Neck H, Kuijpers-Jagtman AM, et al. Stress in Parents of a Child with Hemifacial Microsomia: The Role of Child Characteristics and Parental Coping Strategies. Cleft Palate Craniofac J. 2018;55(7):959-65.

27. Padwa BL, Evans CA, Pillemer FC. Psychosocial adjustment in children with hemifacial microsomia and other craniofacial deformities. Cleft Palate Craniofac J. 1991;28(4):354-9.

28. Volpicelli EJ, Pfaff MJ, Hakimi K, Bradley JP, Solem RC, Lee JC. Age-Related Differences in Psychosocial Function of Children with Craniofacial Anomalies. Plast Reconstr Surg. 2017;140(4):776-84.

29. Collett BR, Speltz ML, Cloonan YK, Leroux BG, Kelly JP, Werler MM. Neurodevelopmental outcomes in children with hemifacial microsomia. Arch Pediatr Adolesc Med. 2011;165 (2):134-40.

30. Speltz ML, Wallace ER, Collett BR, Heike CL, Luquetti DV, Werler MM. Intelligence and Academic Achievement of Adolescents with Craniofacial Microsomia. Plast Reconstr Surg. 2017;140(3):571-80. 
31. Speltz ML, Kapp-Simon KA, Johns AL, Wallace ER, Collett BR, Magee L, et al. Neurodevelopment of Infants with and without Craniofacial Microsomia. J Pediatr. 2018;198:22633 e3.

32. Cohen MS, Samango-Sprouse CA, Stern HJ, Custer DA, Vaught DR, Saal HM, et al. Neurodevelopmental profile of infants and toddlers with oculo-auriculo-vertebral spectrum and the correlation of prognosis with physical findings. Am J Med Genet. 1995;60(6):535-40.

33. Cohen N, Cohen E, Gaiero A, Zecca S, Fichera G, Baldi F, et al. Maxillofacial features and systemic malformations in expanded spectrum Hemifacial Microsomia. Am J Med Genet A. 2017;173(5):1208-18.

34. Johansson M, Billstedt E, Danielsson S, Stromland K, Miller M, Granstrom G, et al. Autism spectrum disorder and underlying brain mechanism in the oculoauriculovertebral spectrum. Dev Med Child Neurol. 2007;49(4):280-8.

35. Stromland K, Miller M, Sjogreen L, Johansson M, Joelsson BM, Billstedt E, et al. Oculo-auriculo-vertebral spectrum: associated anomalies, functional deficits and possible developmental risk factors. Am J Med Genet A. 2007;143A(12): $1317-25$

36. UK Care Standards for the Management of Patients with Microtia and Atresia

37. Johns AL, Im DD, Lewin SL. Early Familial Experiences With Microtia: Psychosocial Implications for Pediatric Providers. Clin Pediatr (Phila). 2018;57(7):775-82.

38. Hamlet CaH, D. Adults' experiences of living with Microtia. A report for Microtia UK.

39. Hamilton KV, Ormond KE, Moscarello T, Bruce JS, Bereknyei Merrell S, Chang KW, et al. Exploring the Medical and Psychosocial Concerns of Adolescents and Young Adults With Craniofacial Microsomia: A Qualitative Study. Cleft Palate Craniofac J. 2018;55(10):1430-9.

\section{CHAPTER 5. SURGICAL TREATMENT}

\subsection{Mandible \& Maxilla \\ Introduction}

Mandibular hypoplasia is seen in $73 \%$ to $91 \%$ of patients with CFM with varying severity $(1,2)$. The Pruzansky classification, later modified by Kaban, has been developed to characterise the mandibular deformity in patients with CFM based on radiographic assessment (3-5). A small but normal shaped mandibular ramus and temporomandibular joint (TMJ) is classified as type I. In type IIa, the mandibular ramus is malformed in size and shape but the TMJ is adequately positioned. Type IIb is characterised by an abnormal shape, size, and location of the mandibular ramus and TMJ. In type III, the ramus, condyle and TMJ are absent. Most patients with CFM have a type I or IIa deformity (respectively $41 \%$ and $26 \%$ ), although severe mandibular hypoplasia, i.e. type IIb or III, is also seen in a considerable number of patients (respectively $14 \%$ and $10 \%$ of all patients with CFM) (2).

Maxillary hypoplasia in patients with CFM could be secondary to the mandibular deformity. A deviation of the mandible to the affected side is seen in patients with CFM, causing facial asymmetry and canting of the occlusal plane (6). Treatment of the maxillomandibular complex to achieve facial symmetry and restore occlusion may be needed.

Besides occlusal problems, other functional problems may occur due to the mandibular/maxillary deformity in CFM. The risk for obstructive sleep apnoea (OSA) increases in patients with mandibular hypoplasia due to obstruction of the upper airway at tongue base level (7). Although OSA may be treated non-surgically, as is described in Chapter 4.1 - Breathing problems, surgical correction of the mandibular deformity may be needed. Besides functional problems, psychosocial problems may occur due to aesthetic concerns related to the facial asymmetry, which may require surgical correction.

To study indications for surgical treatment of the mandibular/ maxillary deformity in patients with CFM and offer recommendations for treatment, the following questions were posed:

5.1.1 What is the indication for surgical treatment of mandibular and maxillary deformity in patients with craniofacial microsomia?

5.1.2 What is the most optimal treatment modality and its timing for mandibular/maxillary deformity in craniofacial microsomia regarding severity, breathing problems, occlusal problems and aesthetics?

The variety of indications for treatment, differences in severity, and diversity in types of treatment make it difficult to standardise care. Nevertheless, recommendations for the indications and types of treatment would help to achieve standardisation in the care process and enable surgeons and patients to make better, wellinformed decisions.

Literature search

A systematic search of literature was performed to identify all available literature on CFM and synonyms. The search was conducted in Embase, Pubmed/Medline Ovid. The full search strategy is reported in the supplementary material.

Inclusion and exclusion criteria:

Type of studies - Original articles

- Systematic review of sufficient quality:

- The question in the systematic review matches the question of the guideline.

- The search of the systematic review was conducted in at least two relevant databases, such as the Cochrane Library, Medline/Pubmed.

- The full search strategy was reported.

- No relevant items were missing in the search strategy.

Type of patients - Patients with craniofacial microsomia

\begin{tabular}{ll}
\hline Subject & - Treatment for mandible/maxilla deformity \\
\hline Exclusion criteria & - Original studies with $<10$ included patients \\
& - Articles published before 1980 \\
& - Case reports \\
& - Expert opinion \\
& - Letters \\
& - Editorials \\
- Narrative reviews
\end{tabular}

Literature was screened on title and abstract. A total of 31 studies were included that addressed (surgical) treatment of mandible or maxilla deformity in patients with CFM. Three systematic reviews were included: Nagy et al. (8), Pluijmers et al. (9), and Van de Lande et al. (10). Two case-control studies by Meazzini et al. were included $(11,12)$. Nineteen retrospective cohort studies were included: Ascenco et al. (13), Gui et al. (14), Kearns et al. (15), Ko et al. (16), Caron et al. (17), Lam et al. (18), Pluijmers et al. (2019) (19), Suh et al. (20), Wang et al. (21), Weichman et al. (22), Zhang et al. (23), Bertin et al. (24), Padwa et al. (25), Tahiri et al. (26), Fattah et al. (27), Yamaguchi et al. (28), and Pluijmers et al. (2018) (29). And seven case series were included: Kaban et al. (1986) (4), Kaban et al. (1988) (30), Rachmiel et al. (2000) (31), Rachmiel et al. 2014 (32), Santamaría et al. (33), Liu et al. (34), and Polley et al. (35). 
An additional search on the treatment for mandibular of maxillary hypoplasia in all types of patients was performed to include any additional literature that might be relevant for patients with CFM. All articles that described outcomes or complications of treatment of mandibular/maxillary hypoplasia were included. Only systematic reviews or meta-analysis were included. The full search strategy is shown in the addendum. A total of 53 articles were identified and seven articles met the inclusion criteria. An additional two articles were found trough a reference check, leading to the inclusion of a total of nine systematic reviews: Breik et al. (2016) (36), Breik et al. (2016) (37), Breik et al. (2016) (38), Ow and Cheung (39), Paes et al. (40), Tsui et al. (41), Verlinden et al. (42), Master et al. (43), and Tahiri et al. (44).

5.1.1 What is the indication for surgical treatment of mandibular and maxillary deformity in patients with craniofacial microsomia?

The literature search for this question was directed towards more specific research questions related to clinical problems, symptoms, treatment options, consequences of not treating, and requirements for surgical treatment of mandibular and maxillary deformity (Appendix 1, http://links.lww.com/SCS/B697). This chapter was divided into psychosocial/aesthetic problems, breathing problems, and occlusal/feeding problems.

\section{Review of literature}

The indication for surgical treatment of mandibular/maxillary deformity in patients with CFM is patient specific and is based on the clinical consequences caused by the deformity, which includes psychosocial/aesthetic problems, breathing problems, or occlusal/ feeding problems.

\section{Psychosocial / aesthetic problems}

No comparative studies assessing the outcomes of surgical treatment of mandibular/maxillary deformity in patients with CFM regarding psychosocial problems have been performed. It is debated in literature whether treatment of the mandibular deformity should be performed in children with CFM without functional complaints, solely to prevent potential negative psychosocial effects. Patients with CFM may experience psychosocial problems, as is discussed in 'Chapter 4.8 - Psychosocial problems'. It is unclear whether treatment of the mandibular/maxillary deformity improves or prevents the psychosocial problems related to CFM. Some authors advocate early correction of the mandibular deformity to prevent potential psychosocial problems $(13,23-25)$. The potential beneficial psychosocial effects of treatment should outweigh the risk of complications and reinterventions, which may be the case in patients with severe mandibular hypoplasia $(9,13)$. If the aim of surgery is to restore symmetry throughout growth, it is important to clearly explain the risks of surgery and the need for subsequent procedures (12). The potential beneficial psychosocial effects of surgical treatment should be "evaluated and justified' by psychologists and not by surgeons or orthodontists, according to Meazzini et al. (12).

\section{Breathing problems}

Bilateral mandibular distraction osteogenesis (MDO) is a preferred method for treatment of respiratory distress / obstructive sleep apnoea in patients ( $<18$ years) with mandibular hypoplasia who are unresponsive to non-surgical treatment. Rachmiel et al. (2014) included 37 patients who underwent MDO for the treatment of OSA over a period of nine year. Fourteen of these patients had CFM, fifteen patients had Pierre Robin sequence and eight patients Treacher Collins. Of the 37 patients included, 21 patients had respiratory distress and 16 patients were tracheostomy dependent
(32). No information on other additional therapy, such as CPAP, was provided. The authors found that mandibular distraction led to improved airway patency in all 21 patients with respiratory distress. All 16 patients who were tracheotomy dependent could be decannulated after MDO (32). No specific outcomes of the included patients with CFM could be extracted. Lam et al. performed a retrospective cohort study to assess the success of MDO on airway problems and included 123 patients who underwent MDO (including 11 patients with CFM) (18). The authors studied all patients who underwent initial MDO between 1995 to 2009. The indication for treatment was to improve upper airway obstruction. Surgical success was defined as the avoidance of tracheostomy due to MDO being performed first or as successful decannulation in patients with a tracheostomy after MDO. The median age at time of MDO was 21 months (IQR 2.2-48.2 months). Patients treated with MDO first had a significant lower mean age compared to tracheostomy first (5.1 versus 30 months, $\mathrm{p}<0.001)$. No specific results of the included patients with CFM could be extracted. Two thirds $(n=42,67.7 \%)$ of the patients with a tracheostomy could be decannulated after MDO and $83.6 \%$ of the patients $(n=51)$ did not need a tracheostomy after MDO. The surgical success of MDO in patients who had a tracheostomy first was found to be the lowest in patients with CFM compared to other syndromic conditions including Pierre Robin sequence or Treacher Collins syndrome (OR: 0.05 (CI 0.005-0.43), $\mathrm{p}=0.007$; adjusted for sex and age). Due to these lower odds of success, the authors recommend that patients with CFM and respiratory distress who are unresponsive to non-surgical treatment should have a tracheostomy as an initial procedure and MDO secondarily to achieve decannulation (no specific age for treatment was proposed) (18). MDO as treatment for OSA in patients with CFM and unilateral mandibular hypoplasia was found to have a much lower success rate of $36.4 \%$ as only four of the eleven treated patients had normal PSG after MDO. The reason for this low success rate was not reported (e.g. bad timing or quality of the MDO). It is relevant to be aware that decannulation may not be achieved (17).

The additional search on MDO in non-CFM patients led to the inclusion of five systematic reviews on the treatment of MDO to improve respiratory distress. Breik et al. included 51 articles on MDO outcomes in their meta-analysis and studied 490 patients with micrognathia who underwent bilateral MDO (mean age: 10.4 months, range 5 days to 8 years) (37). All patients were treated for upper airway obstruction and had unsuccessfully undergone non-surgical therapy. A successful outcome of MDO was defined as the relief of upper airway obstruction or prevention of tracheostomy. They found that MDO was successful in $95.5 \%$ of the patients and that $4.5 \%$ (22 patients) required a tracheostomy. Most patients with a failed outcome had undiagnosed lower airway anomalies including laryngomalacia and tracheal or subglottic stenosis, or central apnoeas (19/22). This suggests an incomplete pre-operative work-up as these factors should have been noted before starting MDO. Of the patients with a tracheostomy $(n=152), 80.3 \%$ $(n=122)$ could be decannulated after MDO. The follow-up varied between the studies included, although all studies had a minimum follow-up of one year. The authors state that, due to the variation in follow-up, the outcomes could only be interpreted as short-term (up to one year). All patients considered for MDO should have polysomnography and nasoendoscopy to confirm the upper airway obstruction and rule out lower airway anomalies, according to Breik et al. (37). The success rate of MDO to achieve decannulation is higher in patients age $<24$ months compared to older patients. No differences in age on the success rate of primary MDO were found (37). The rate of successful MDO in patients with micrognathia as prevention of tracheostomy was $89 \%-91.3 \%$; and the rate of decannulation after MDO was $78.4 \%$ - $94 \%$ in the other systematic reviews included $(39,40,44)$. The follow-up after treatment varied 
in the studies included: Tahiri et al. reported a mean follow-up time of 2.4 years, Paes et al. reported a follow-up time $>12$ months in nine of their twelve included articles, and Ow and Cheung did not report the time of follow-up $(39,40,44)$. Tsui et al. found a significant improvement in apnoea-hypoapnoea index scores (AHI) after MDO, which successfully corrected the OSA in 90$100 \%$ of the patients (41).

\section{Occlusal / feeding problems}

The prevalence of feeding problems in CFM is $26 \%$ to $63 \%$ and is further analysed in 'Chapter 4.2 - Feeding problems' $(45,46)$. Many factors may lead to feeding problems, including chewing problems which may be caused by mandibular hypoplasia. However, the precise role of mandibular hypoplasia in feeding problems in CFM is not described in literature. Liu et al. and Fattah et al. emphasise the importance of orthognathic surgery to restore facial symmetry and improve the occlusal cant in patients with $\operatorname{CFM}(27,34)$. Tahiri et al. advises to restore significant occlusal abnormalities in patients with CFM and severe mandibular hypoplasia (Pruzansky IIB, III) with a costochondral graft (26). Nevertheless, no literature is available on the exact indication for surgical treatment of occlusal problems in CFM.

Breik et al. studied 21 articles, including 300 non-CFM patients with micrognathia and feeding problems (mean age seven months, range five days - six years) (38). After treatment with bilateral MDO, $82 \%$ of the children $(n=246)$ were able to feed completely orally within twelve months of treatment (mean follow-up three years, range one to seven years). These patients did no longer need feeding adjunct and could be fed exclusively orally. The authors hypothesise that this is due to an increase in upper airway continuity and possibly due to improved lip closure creating a better-quality suckling reflex. Additionally, four articles on gastro-intestinal reflux disease (GERD) were studied and the authors found that 66 of the 70 patients (94\%) with GERD (based on $\mathrm{pH}$ monitoring) had no reflux after treatment with bilateral MDO. The authors conclude that early MDO is successful in treating feeding problems in patients with micrognathia. However, they note that upper airway obstruction should first be treated with non-surgical management, which could be followed by surgical treatment if non-invasive techniques fail (38).

Conclusions

Level 3 Indications for surgical treatment of the mandibular and/or maxillary
deformity in patients with craniofacial microsomia include:
- Breathing problems
- Feeding / occlusal problems.
$\begin{aligned} & \text { - Psychosocial / aesthetic considerations } \\ & \operatorname{Ref}(9,12,13,17,18,23-27,34)\end{aligned}$

Level 3 Bilateral mandibular distraction osteogenesis (MDO) appears to be an effective treatment in patients with micrognathia, respiratory distress and a tracheostomy (mean age range two months to five years). - Most patients with a tracheostomy can be decannulated after bilateral MDO: 78\% - 94\% ('tracheostomy first' group).

- Some patients still require a tracheostomy after initial treatment with bilateral MDO: $5 \%-11 \%$ ('MDO first' group).

Large differences in outcomes of MDO in patients with craniofacial microsomia for treatment of breathing problems are observed: - The success rate of mandibular distraction osteogenesis for obstructive sleep apnoea in patients with unilateral craniofacial microsomia appears to be low (36.4\%).

- The success rate of decannulation after mandibular distraction osteogenesis may be lower in patients with craniofacial microsomia compared to other craniofacial syndromes including Pierre Robin sequence or Treacher Collins syndrome (reported in a single study including 62 patients ( 7 with CFM) in the tracheostomy first group; OR 0.05 , CI $0.005-0.43)$.

$\operatorname{Ref}(17,18,37,39-41,44)$

Level 3 Bilateral mandibular distraction osteogenesis (MDO)
appears to be an effective treatment in patients with
micrognathia and feeding problems:
- $82 \%$ of the patients $(\mathrm{n}=246 / 300)$ in need of
feeding adjuncts were able to feed exclusively orally
within twelve months after bilateral MDO.
$\operatorname{Ref}(38)$

\section{Considerations}

\section{- Overall quality of evidence}

The conclusions on patients with CFM were based on studies on retrospective cohort studies, case series or a systematic review of these types of studies. Since all studies were non-comparative studies, the quality of evidence was graded on level 3. The conclusions which were based on the systematic reviews (included after an additional search) were graded on level 3, since almost all studies included in the systematic reviews were retrospective cohort studies and other non-comparative studies. In addition, it should be kept in mind that this evidence is based on patients with micrognathia and not specifically on patients with CFM. This indirectness of evidence also leads to a lower quality of evidence.

\section{- Balance of benefits and harms}

No literature on the psychosocial consequence of the mandibular deformity specifically is available. However, some patients experience difficulties due to their different facial appearance compared to other children/persons (47). Treatment of the mandibular/maxillary deformity may help to improve self-esteem or prevent psychosocial problems. Additionally, mandibular hypoplasia can cause breathing and feeding/occlusal problems. The potential harms of these problems and need for therapy are further explained in 'Chapter 4.1 - Breathing problems' and 'Chapter 4.2 - Feeding problems'. The burden of surgical treatment of the mandibular/ maxillary deformity and its potential risks are important to acknowledge. The benefits of (early) treatment should outweigh the risks. The risk for re-surgery, nerve or dental damage, scar formation, insufficient bone quality, infection, etc. should be taken into account. Therefore, conservative treatment for breathing/feeding problems in CFM is considered the first choice of treatment, which can be followed by surgical treatment if conservative therapy fails.

\section{- Outcome importance}

No literature is available on the psychosocial consequences of mandibular and maxillary deformity in patients with CFM. Nevertheless, aesthetic difficulties could cause psychosocial problems which may be prevented or treated by surgical correction of the mandibular/maxillary deformity. Besides aesthetic concerns, breathing or feeding problems could make surgical intervention necessary. Mandibular hypoplasia could lead to obstructive sleep apnoea, which may have severe consequences for growth, daily functioning and (cognitive) development. Feeding problems can lead to impaired growth and development. Presence of these functional problems calls for surgical treatment of the mandibular/maxillary deformity in patients with CFM, especially if conservative treatment fails.

\section{- Costs and resources}

The impact on costs and resources of the given recommendations will vary per member state, depending on the available care 
providers and facilities. The recommendations concern the essential requirements for adequate treatment of patients with CFM and should thus be implemented. Costs are lowest and resources are most efficiently used when care for craniofacial disorders is centralised in a limited number of expert centres per member state. A general rule that can be applied is one expert centre per 10 million inhabitants.

\section{- Inequity of the recommendation}

The goal of the European Reference Networks (ERNs) is to eliminate inequality within Europe with regard to care for patients with rare diseases. At present, not every member state offers an expert centre for CFM, or the level of provided care does not (yet) meet all the requirements that are outlined in this guideline. By defining the baseline of required care for CFM, this guideline will help these member states to reach the appropriate level. The ERN on craniofacial anomalies and ENT disorders (ERN-CRANIO) can guide a patient in Europe to the available centres of expertise (www.ern-cranio.eu) and can support care providers with diagnosis and treatment advice.

\section{- Feasibility of the recommendation}

Recommendations refer to the general requirements for delivering optimal healthcare and are discussed with members from participating European countries. Quality of care was paramount in the discussions. Centralisation is proposed as one of the core values. However, in some countries the national organisation of healthcare might impede centralisation. National implementation of the ERNs that fits the situation of each country is necessary. For the member states with the lowest number of inhabitants, the establishment of a craniofacial centre might not be feasible, and collaboration with a craniofacial centre in the surrounding countries can be an option.

\section{- Acceptability of the recommendation}

It is expected that all stakeholders want to apply and will be applying the recommendations, because they are employed in ERNacknowledged institutions. National implementation plans are necessary to ensure that recommendations fit the situation in each country. In addition, not all countries participating in the ERNCRANIO are represented in the guideline development group, and new members will join within the coming years. For these countries, acceptance and implementation of ERN guidelines such as this guideline on CFM is obligatory.

\section{Rationale of the recommendations}

A patient-specific approach is needed for surgical treatment of mandibular/maxillary deformity in patients with CFM due to the various indications for treatment, including psychosocial/aesthetic, breathing problems and feeding/occlusal problems. Providing information about the treatment options and their benefits and harms is important for patients to make a well-informed decision and have a realistic view of what can be expected, especially in patients with aesthetic concerns. For all patients with aesthetic difficulties, as mentioned in other chapters of this guideline, psychosocial help is important before starting with (surgical) treatment. In patients with functional problems (i.e. breathing, feeding/occlusal), there might be a strong indication for surgical correction of the mandibular/maxillary deformity to prevent worse outcomes, especially if conservative treatment fails. The choice for treatment is based on clinical assessment and should be discussed in a multidisciplinary team, taking all other (future planned) procedures into account.

\section{Recommendations}

- Consider surgical management (tracheostomy, adenotonsillectomy, mandibular and/or maxillary surgery) in patients with craniofacial microsomia for the treatment of breathing problems if non-surgical therapy fails or to end non-surgical therapy.

- Inform patients and parents about of the uncertainty of respiratory outcomes following mandibular and/or maxillary surgery for OSA in patients with CFM.

- If surgical treatment of the mandibular/maxillary deformity in patients with craniofacial microsomia is indicated to prevent or treat psychosocial problems, it is important to inform the patient about the potential benefits and harms and to ensure that the patients/ parents have a realistic view of what can be expected.

- It is advised to integrate the (surgical) treatment of the mandibular/maxillary deformity in patients with craniofacial microsomia in the planning of other surgeries, especially for those that affect facial symmetry, palsy, soft tissue augmentation and treatment of atresia or microtia.

\section{Research gap}

Future studies on the indication for mandibular distraction in patients with CFM could help determine an optimal treatment strategy. Literature on the impact of mandibular/maxillary surgery on psychosocial problems due to aesthetic concerns is missing. Multicentre prospective cohort studies with long-term outcome measures and an assessment of the impact of surgery on psychosocial health could offer insight into the indication for MDO.

5.1.2 What is the most optimal treatment modality and its timing for mandibular/maxillary deformity in patients with craniofacial microsomia regarding severity, breathing problems, occlusal problems and aesthetics?

The literature search for this question was directed towards more specific research questions related to treatment options, complications, advantages and disadvantages, short and long term results and treatment burden for treating mandibular/maxillary deformity in patients with CFM (Appendix 1, http://links.lww.com/SCS/B697). This review of literature addresses the most optimal treatment modalities related to the severity of mandibular/maxillary deformity, breathing problems, occlusal problems and aesthetics. Included patient outcomes were related to breathing problems, occlusal problems and aesthetics. This chapter was subdivided into the type of mandibular/maxillary surgery in patients with CFM, bilateral mandibular distraction osteogenesis (MDO), unilateral MDO, internal or external distractor for MDO, complications of MDO, mandibular reconstruction with bone graft, free bone flap, orthognathic surgery, and temporomandibular joints (TMJ) implants. Additional literature on complications of MDO and distractor type for MDO was included through an additional search on non-CFM patients.

\section{Review of literature Types of surgery}

In a systematic review, Pluijmers et al. studied the outcomes of surgical correction of the mandible in patients with unilateral CFM (9). A total of 19 articles were included with a mean follow-up of 4.3 years after treatment. Mandibular reconstruction (43.9\%) and distraction $(13.7 \%)$ with a costochondral bone graft, or less frequently used iliac crest or rib grafts, were the most common types of 
surgery used in patients with severe mandibular hypoplasia (Pruzansky type IIB and III). These patients often needed reconstruction of the mandibular ramus and/or temporomandibular joint. Patients with mild mandibular hypoplasia (Pruzansky I and IIA) were generally treated with distraction osteogenesis $(30.4 \%)$ or osteotomies $(2.1 \%)$. The indications for surgery were not reported. Most of the studies on mandibular distraction and bone grafts that were included in the systematic review by Pluijmers et al. showed a recurrence in facial asymmetry after treatment in patients with severe mandibular hypoplasia (Pruzansky II-III), which may be due to resorption, relapse, or asymmetric growth (9). More stable results were seen in patients with mild mandibular hypoplasia (Pruzansky I, IIA), receiving distraction osteogenesis or osteotomies. Distraction osteotomies with bone grafts led to the most stable long-term results for both mild and severe mandibular hypoplasia. The authors conclude that the outcomes of mandibular correction in patients with unilateral CFM are 'not so much treatment-dependent, but more patient-dependent'. In patients with mild mandibular hypoplasia, a single-stage surgical correct might be sufficient to correct the facial asymmetry, compared to patients with severe mandibular hypoplasia who benefit from a multi-stage treatment protocol. The psychosocial benefits of treatment must outweigh the risks of treatment. Therefore, Pluijmers et al. advise to perform single-stage correction after the permanent dentition phase or in skeletal mature patients (9).

A large retrospective cohort study of 565 patients with CFM showed that $33.5 \%$ of all patients with CFM had mandibular surgery (19). The percentage of patients that needed mandibular surgery increased with the severity of mandibular hypoplasia: $5 \%$ of the patients with Pruzansky I, 22\% of the patients with Pruzansky IIA, $51 \%$ of the patients with Pruzansky IIB, and $68 \%$ of the patients with Pruzansky III had mandibular surgery. The types of surgery also varied with the severity: osteotomies, genioplasties and MDO were most frequently performed in patients with mild mandibular hypoplasia (Pruzansky I and IIA), whereas MDO followed by mandibular reconstruction with a bone graft or initial reconstruction of the condyle with a bone graft were most often performed in patients with severe mandibular hypoplasia (Pruzansky IIB and III). Complications occurred in $33 \%$ of the patients who had mandibular reconstructions and included graft infections, postoperative hypoesthesia, (graft) ankylosis, infection, or malposition. Additional surgeries were most frequently needed in patients with severe mandibular hypoplasia and often included bimaxillary or sagittal split osteotomies and genioplasties. Correction of the maxilla was needed in $13 \%$ of all patients with CFM and was always performed at the end of puberty. Treatment included bimaxillary osteotomies and Le Fort 1 procedures. Patients with bilateral CFM had a higher number of surgical interventions compared to patients with unilateral CFM (average 2.44 versus $1.85, \mathrm{p}=0.007$ ). The total number of surgeries is influenced by the age at which the first surgical intervention was performed. Linear regression analysis showed a higher number of operations for every year decrease in age, independent of the severity of mandibular hypoplasia. According to the authors this suggests it is beneficial to perform mandibular corrections at an older age, unless functional problems warrant earlier treatment (19).

\section{Bilateral mandibular distraction osteogenesis}

Bilateral MDO is considered to be an effective treatment modality for breathing problems in patients with micrognathia, as is further described in the previous Chapter 5.1.1. - Indications for mandibular surgery. In the literature on MDO, the highest quality of evidence is based on large systematic reviews on patients with retrognathia/micrognathia. Although this evidence is indirect as it is based on patients with micrognathia and not CFM, studies that included patients with CFM showed similar results $(17,18,32)$. As described in the previous chapter, results of MDO for the treatment of OSA in patients with unilateral CFM may be associated with lower success rates (17).

\section{Unilateral mandibular distraction osteogenesis}

The long-term stability of MDO after early osteodistraction in patients with unilateral CFM was studied in a systematic review by Nagy et al. (8). A total of 13 studies (155 patients) on unilateral MDO aimed to correct asymmetry in patients with CFM that was performed before the age of 16 were included. The mean age at the time of MDO was $7.8 \pm 2.6$ years, with a mean follow-up of $4 \pm 3.5$ years. The effectiveness of MDO was based on the long-term stability of mandibular dimensions. Although seven studies showed stable results after MDO and six studies did not, most studies could not prove long-term stability or were based on non-objective evaluation methods. Especially in patients with a Pruzansky IIB or III deformity, the facial asymmetry and affected ramus height are unpredictable after unilateral MDO.

Nagy et al. found no evidence for long-term stability of unilateral MDO in patients with CFM (8). Revisional surgery is often indicated after early MDO to maintain facial symmetry during growth, indicating unstable long-term results $(8,19)$. This was also found in the systematic review by Pluijmers et al., which showed an increase in facial asymmetry after MDO and placement of bone grafts in Pruzansky II-III patients. However, patients with mild mandibular hypoplasia (Pruzansky I-IIA) showed more stable results (9). Ascenco et al. found that $90 \%(n=30)$ of their studied patients had recurrence of their facial asymmetry after unilateral MDO (13).

Nagy et al. concluded that literature shows that CFM is a nonprogressive disease (8). The facial and mandibular asymmetry in patients with CFM does not increase over time. However, some authors advocate early unilateral MDO to stimulate midfacial growth of the affected side, which would lead to better facial symmetry and fewer surgical interventions during development $(4,15,30)$. Kaban et al. showed that creating an open bite on the affected side during childhood could lead to vertical growth of the maxilla and alveolar bone $(4,30)$. Additional surgery was not needed in some patients with mild mandibular hypoplasia (4 of the 17 patients). Early unilateral MDO was also advocated by Weichman et al. (22). They studied the long-term outcomes of unilateral MDO ( $16.5 \pm 4.2$ years) in patients with mild to moderate unilateral CFM (Pruzansky I and IIA). Their retrospective analysis of 19 patients showed satisfactory results (based on randomised photographic judgement by a panel) in 12 patients and unsatisfactory results in 7 patients. Patients with satisfactory results appeared to be treated at a younger age compared to the unsatisfactory group (4.7 years versus 7.5 years, $\mathrm{p}=0.07$ ). Additionally, the average percentage of overcorrection was greater in the satisfactory cohort compared to the unsatisfactory cohort $(41.3 \%$ compared to $1.8 \%)$ (22). However, based on these data it cannot be concluded that overcorrection leads to better outcomes. Overcorrecting has been proposed to compensate for any relapse or lack of growth on the affected side. However, this has its limits since it creates a contralateral crossbite leading to occlusal problems, it may increase facial asymmetry by overcorrecting the position of the chin, and it does not prevent the need for additional surgery (8).

Ko et al. compared the long-term facial growth of 20 patients with unilateral CFM: 9 patients had early MDO (age 5-9 years) and 11 patients without early MDO or other reported treatment (mean follow-up 13.08 years) (16). All patients had a Pruzansky grade II mandible deformity. The indication for MDO was to correct facial 
asymmetry, to treat occlusal problems, or to treat psychological/ aesthetic difficulties. The growth of the mandible was lower on the affected side compared to the unaffected side and showed a similar growth pattern to that of untreated patients. The mandibular ramus length reduced from $90 \%$ direct after MDO to $69 \%$ after follow-up. Nevertheless, early MDO may lead to a smaller chin deviation in the long term $(8.42 \mathrm{~mm}$ versus $12.7 \mathrm{~mm})(16)$. A retrospective study by Suh et al. with 10-year follow-up showed no differences in mandibular, maxillary, and occlusal tilting after early unilateral MDO $(\mathrm{n}=26$ patients) (20). The changes of mandibular dimensions after treatment gradually returned asymmetrical with growth in all treated patients (20). Meazzini et al. showed in a comparative study of 24 patients with unilateral CFM (all Pruzansky I and II) that the results of early MDO were temporary and the ramus length returned to values almost identical as prior to treatment (12). However, adding functional orthodontic stimulation after unilateral MDO may lead to less canting of the occlusal plane although no differences in ramus length were seen (11). This conclusion is based on the results of a study by Meazinni et al. with a very small sample size (ten patients treated with functional orthodontic stimulation and seven without) (11).

Most patients with CFM need orthognathic surgery after early MDO to treat the recurrent facial asymmetry and/or occlusal problems (13). The benefits of early MDO, which may yield positive psychosocial effects, should be balanced with the need for later surgery (13). Zhang et al. compared the need for orthognathic surgery in 17 patients who had early MDO and in 21 patients without MDO (23). The severity of the mandibular deformity in both groups was similar. No differences between the MDO and nonMDO group were seen with regard to the need for orthognathic surgery: $59 \%$ of the MDO group and $38 \%$ of the non-MDO group needed orthognathic surgery $(p=0.203)$. Patients who needed repeated MDO (six patients, $35 \%$ ) appeared to have a higher rate of additional orthognathic surgery $83.3 \%$ ) compared to patients who had single MDO (45.5\%) or no MDO (38.8\%) $(\mathrm{p}=0.162)(23)$.

\section{Internal or external distractor}

The outcomes of the use of an external and internal distractor for bilateral MDO in patients with CFM were studied by Rachmiel et al. in two different case series, including 37 patients in total and 14 patients with CFM $(31,32)$. The age at time of treatment varied from 6 months to 14 years. Outcomes of treatment, defined by mandibular advancement and improved airway, were successful in all patients treated with both external and internal distractors. Placement of external distractors was considered simpler and permitted longer distraction compared to internal distractors (31, 32 ). However, loosening of the pins (22.5\% of the patients), pin tract infections ( $16.7 \%-27.5 \%$ of the patients), and earlier removal of the pins ( $15 \%$ of the patients) was reported in some patients. Relapse after distraction was higher after external versus internal distraction $(23.5 \%$ versus $13.3 \%)$, which may be due to bending of the pins. Additionally, scars on the buccal skin were notable after external distraction. Internal distractors led to stable distraction with precise and predictable vector of lengthening in most patients, although one patient $(5.8 \%)$ had a device failure which led to an additional operation to remove the device. No facial nerve damage was seen after external distraction, although internal distraction led to transient damage to the mandibular branch of the facial nerve in $14.7 \%$ of the patients. Local infection of internal distractors was seen in two patients $(5.8 \%)$. In addition, two patients had a contralateral open bite after internal distraction due to reduced vector control, which had to be corrected with orthodontic treatment. Another important aspect of internal distraction is the need for a second operation under general anaesthesia to remove the distractor. The authors advocate the use of internal distractors whenever possible due to less discomfort and less visible scars, and a lower risk of pin loosening and relapse. Nevertheless, external distraction could be used in patients with severely hypoplastic mandibles or when optimal vector control is necessary $(31,32)$.

Potential differences in outcomes of MDO with internal or external distractors were studied in a systematic review by Breik et al., which was included after our additional search (36). A total of 43 articles on surgical outcomes of bilateral MDO in patients with micrognathia were included. A similar number of patients was treated with internal $(n=207)$ and external $(n=206)$ distractors. The age of treatment and potential differences in timing of treatment with either internal or external distractors was not reported. The number of device-related failures was significantly lower in patients treated with internal distractors (two failures) compared to external distractors (thirteen failures) $(p=0.012)$. Failure of the device was often caused by loosening of the pins during distraction. A higher number of complications occurred in patients treated with external distractors (33\%) compared to internal distractors $(23 \%)$, which was also observed in a systematic review by Tahiri et al. (22.1\% external distractor versus $8.3 \%$ internal distractors) (44). Significant scarring $(9.4 \%$ versus $0.61 \%, \mathrm{p}=0.006)$ and technical failures $(8.72 \%$ versus $3.05 \%), p=0.39)$ were more frequently seen in patients treated with external distractors compared to internal distractors (36). However, all cases with infections that needed surgical drainage $(n=3)$ were seen in the internal distractor group.

\section{Complications of mandibular distraction osteogenesis}

Wang et al. studied complications of MDO in 71 patients with unilateral CFM (21). All distractors were internal and placed in patients aged 5 to 14 years (mean follow up: 2.75 years). The overall complication rate was $36.6 \%$. Minor complications, which could be resolved without invasive treatment, were seen in $18.3 \%$ of the patients and included local soft tissue infections $(\mathrm{n}=11)$ and neurapraxia $(\mathrm{n}=2)$. Moderate complications were reported in $12.7 \%$ of all patients and required invasive treatment. This included temporary resorption of the temporal bone $(n=5)$, loosening of the device $(n=3)$, and hypertrophic scars $(n=1)$. Temporary resorption of the bone was considered a remarkable complication and may have been caused by an elongated distractor, which led to the formation of a hole in the temporal bone. Major complications, which not could be resolved with invasive therapy, were seen in 4 patients $(5.6 \%)$. This included tooth or tooth germ damage $(n=2)$ and long-term limitations of mouth opening due to ankylosis $(n=1)$ and coronoid process hyperplasia $(\mathrm{n}=1)$. Tooth germ damage was expected to occur and was considered inevitable due to an extreme short mandibular ramus. Complications of MDO were seen in 4 $(23.5 \%)$ of the 17 patients with CFM studied by Zhang et al. and included ankylosis, distractor replacement due to malfunction, and re-surgery due to premature fusion of the mandible (23).

The additional search to identify literature on distraction osteogenesis in non-CFM patients led to the inclusion of four systematic reviews that described the risk of complications after bilateral MDO. Verlinden et al. found an overall complication rate of $34.4 \%$ in 1,258 treated patients (42). Again, the age at which treatment was initiated was not reported. Most complications resolved spontaneously or could be treated (15\% of all patients). This included temporary hypoesthesia, local infection, dehiscence, pin loosening, or incorrect vector. Technical complications, such as device failure, pin loosening or incomplete osteotomy were seen in in $4 \%$ of the patients. Permanent disabling complications or unsatisfactory results were reported in $7.9 \%$ of all patients and included permanent teeth damage or facial nerve palsy, TMJ ankylosis, non-union, hypertrophic scars, and a 
skeletal open bite. In another systemic review on complications of MDO, Master et al. reported a complication rate of $20.5 \%$ to $25.6 \%$ (43). Most seen complications were: relapse (64.8\%), tooth injury (22.5\%), hypertrophic scars $(15.6 \%)$, nerve injury $(11.4 \%)$, infection $(9.5 \%)$, incorrect vector $(8.8 \%)$, device failure $(7.9 \%)$, non/malunion $(2.4 \%)$, and TMJ injury (0.7\%) (43).

Tahiri reported a slightly lower complication rate of $23.8 \%$ in a total of 711 patients, which mainly included abscess formation, nerve injury, hypertrophic scar formation, and a skeletal open bite (44). Interestingly, the authors found that complications mainly occurred in patients who were treated at an older age. The mean age of patients who experienced complications was 1.5 years older than the mean age of all patients (36.9 months versus 18.1 months).

Breik et al. found in their systematic review that patients treated with a higher distraction rate of $2 \mathrm{~mm} /$ day or 1.1 - to $1.9 \mathrm{~mm} /$ day, had a lower incidence of complications compared to $1 \mathrm{~mm}$ /day distraction (respectively $28.6 \%$ and $26.5 \%$ versus $36.7 \%$ complications, number of patients per distraction rate respectively 199,113 , and 143) (36). The mean age of patients treated with a distraction rate of $2 \mathrm{~mm}$ /day was significantly younger (9.9 months) compared to patients with a $1 \mathrm{~mm} /$ day distraction rate (38.6 months). The types of complications, which included infection, ankylosis, scarring, dental injury, and premature ossification were similar between the three distraction rates groups. However, facial nerve injuries were less frequently observed in patients with a distraction rate of $>2 \mathrm{~mm} /$ day compared to $1 \mathrm{~mm} /$ day $(\mathrm{p}<0.018)(36)$. The risk for transient hypoesthesia of the inferior alveolar nerve was found be greater in patients with a distraction rate of $>1 \mathrm{~mm} /$ day $(19.5 \%$ versus $2.4 \%$ in patients with $1 \mathrm{~mm} /$ day distraction) $(43,48)$. The authors concluded that rapid distraction with $2 \mathrm{~mm} /$ day is favourable and safe in children younger than 12 months of age, due to the shorter period of treatment (36).

\section{Mandibular reconstruction with bone graft}

The systematic review of Pluijmers et al. on mandibular reconstruction in CFM showed that patients with severe mandibular hypoplasia often need reconstruction of the ramus and TMJ (9). The most popular graft was the costochondral graft, follow by iliac crest, rib graft, and fibular free flap. In patients with mild mandibular hypoplasia (Pruzansky I and IIA), bone grafts were sometimes used in combination with MDO or as interposed bone grafts for mandibular lengthening (9). More than $50 \%$ of the patients treated with bone grafts showed a recurrence of facial asymmetry, which often led to additional surgery. In a retrospective cohort study of 33 patients with CFM treated with costochondral grafts, Padwa et al. showed that patients with a lower O.M.E.N.S. score had better outcomes of treatment (25). These patients had symmetrical and equivalent growth of the reconstructed ramus compared to the healthy side, in contrast to more severely affected patients who did not (25).

Tahiri et al. studied 22 patients with CFM who had mandibular reconstruction with a costochondral graft (26). The mean age at treatment was 7 years (range 2 - 15 years) and follow-up was 6.75 years (range 7 months - 15 years). No failures of the bone grafts, no malunion or non-union, and no donor-site complications were reported. Adequate, symmetrical growth was seen in $81.8 \%$ of the hemimandibles, whereas $18.2 \%(n=6)$ of the costochondral grafts grew insufficiently. These patients required additional distraction osteogenesis to correct mandibular asymmetry. Ankylosis of a rib graft was seen in one patient, which was in retrospect the only patient who was treated with an osseous rib graft without cartilage. This led to a modification of the technique by always using a costochondral graft with a cartilage cap. The authors advised to perform mandibular reconstruction with rib grafts from the age of five, since the quality of the rib is sufficient at this time (26), or during mid-mixed dentition phase (8 to 10 years) according to Nagy et al. and Padwa et al. $(8,25)$.

Mandibular occlusal canting was improved in all patients after treatment with a costochondral graft in a retrospective cohort study of 39 patients with CFM (treated at a mean age of 13 years) (24). However, a slight recurrence of mandibular asymmetry was seen at follow-up. Aesthetic outcomes were reported to be good. Complications of costochondral grafting included post-surgical infections $(n=2)$, lower labial facial nerve paresis $(n=2)$, hypertrophic scarring $(\mathrm{n}=1)$, and costochondral graft overgrowth $(\mathrm{n}=2)$. Secondary surgery due to occlusal canting relapse or chin deviation was needed in $23 \%$ of the patients (24).

Complications after mandibular reconstruction occurred in $33.3 \%(n=21)$ of the 63 patients retrospectively studied by Pluijmers et al. (19). This included autologous graft infections $(n=12)$, postoperative hypoesthesia $(n=9)$, graft ankylosis $(n=8)$, ankylosis $(n=8)$, wound infection $(n=6)$, malunion/non-union $(n=5)$, rib overgrowth $(n=3)$, and graft dehiscence and hypertrophic scars $(n=1)$.

\section{Free bone flap}

Mandibular reconstruction with a free flap may be an option for patients with severe mandibular hypoplasia (Pruzansky IIB and III) in which MDO is not feasible. Santamaría et al. reported successful mandibular reconstruction with a vascularised fibula flap in 9 of the 10 included patients (mean age 7.2 years, range $3-10$ ) (33). Facial symmetry, mastication and patient satisfaction improved after treatment (mean follow-up 3.75 years, range 1-8 years). Two patients had minor donor site complications including dehiscence and partial skin paddle necrosis. No extremity weakness, ankle stiffness or gait disturbances were observed. Half of the patients had prior reconstruction with bone grafts that failed. The ideal age of reconstruction was considered to be between 5 and 7 years, since harvesting of the fibula and the microsurgical procedure is more challenging in younger patients.

\section{Orthognathic surgery}

Orthognathic surgery, including bimaxillary osteotomies, Le Fort I osteotomies, sagittal split osteotomies, and genioplasties, is often indicated in patients with CFM. This may be as additional treatment in patients with severe mandibular hypoplasia or bilateral CFM, or as initial treatment in patients with mild mandibular hypoplasia (19). The aim of treatment is to level the occlusal cant, achieve a good occlusal relationship, and align the dental midline to the facial midline $(19,27)$. Since CFM is considered to be a nonprogressive disorder, treatment may be delayed until skeletal maturity to achieve stable results (27). Facial symmetry and occlusal outcomes improved after orthognathic surgery in 10 patients with CFM studied by Fattah et al. and remained stable at long-term follow-up (3 years, range $1.1-7.75$ years). Bertin et al. reported that all their 39 included patients who had mandibular reconstruction needed restoration of the occlusal canting at skeletal maturity (24). Orthognathic surgery may be combined with fat grafting when performed in adulthood, to improve the facial deformity in patients with CFM (28). Especially in patients with a notable soft tissue deficiency, orthognathic surgery alone may not be sufficient to achieve facial symmetry (28).

Surgical correction of the maxillary deformity in patients with unilateral CFM was studied in a systematic review by Van de Lande et al. (10). Seven articles including a total of 57 patients, with at least 6 months follow-up after treatment were included. Patients had either a bimaxillary osteotomy (20 patients, mean age of treatment 19.7 years) or a Le Fort I osteotomy with MDO (37 patients, mean 
age of treatment 19.9 years). No surgical correction of exclusively the maxilla was reported. Bimaxillary osteotomies were more frequently performed in patients with severe mandibular hypoplasia, whereas a Le Fort I osteotomy with MDO was more often reported in patients with mild mandibular hypoplasia. Similar results were seen in a retrospective cohort study by Pluijmers et al., who showed that nearly half of the patients who had a bimaxillary osteotomy had prior mandibular surgery, especially patients with severe mandibular hypoplasia (Pruzansky III) (29).

All of the included studies in the systematic review by Van de Lande et al. showed improvement of the occlusal cant after maxillary surgery, although four articles reported a slight residual cant of a maximum of 2.3 degrees (10). Improvement of facial symmetry and aesthetic outcomes, based on patients' or surgeon's opinion, was reported in four studies. Most studies advised to surgically correct the maxillo-mandibular asymmetry combined with orthodontic pre-treatment at skeletal maturity or permanent dentition, on the condition that no functional or psychological problems are present $(10,24,27,29)$.

\section{Temporomandibular joint (TMJ) implants}

Polley et al. presented a case series of ten patients with CFM and a Pruzansky III mandibular deformity who had custom-made titanium TMJ implants placed at skeletal maturity (35). The follow-up was six months to four years. Treatment led to stabilisation of the occlusal relationship, maintained or improved mouth opening, and improved facial aesthetics. No permanent facial nerve paresis, no infection, and no implant incidents or dislocation was recorded. Placement of TMJ implants at skeletal maturity appears to be a solution for patients with failed autogenous mandibular reconstructions (35).

\section{Conclusions}

Level 3 Mandibular surgery
A third of all patients with craniofacial microsomia require
mandibular surgery.
Mandibular and facial asymmetry in patients with craniofacial
microsomia is non-progressive.
Most stable long-term results of mandibular surgery in patients
with craniofacial microsomia are seen in patients with mild-
moderate mandibular hypoplasia (Pruzansky I-IIA) compared
to severe mandibular hypoplasia (Pruzansky IIB-III).
The total number of mandibular/maxillary surgeries increases if
patients are treated at a younger age, independent of the
severity of the mandibular hypoplasia.
Ref $(4,8,9,19,30)$

\section{Level 3 Mandibular distraction osteogenesis (MDO)}

MDO is often (approximately 30\%) performed in craniofacial microsomia patients with mild-moderate mandibular hypoplasia (Pruzansky I - IIA).

Bilateral MDO in patients with micrognathia and respiratory distress has a high success rate and may prevent tracheostomy or lead to decannulation. Success rates may be lower in patients with unilateral CFM. (See Chapter 5.1.1)

The outcomes of early unilateral MDO in patients with craniofacial microsomia and severe mandibular hypoplasia (Pruzansky IIB-III) regarding facial asymmetry and affected ramus height are unpredictable.

The long-term stability of early unilateral MDO (performed $<16$ years) in patients with CFM appears to be poor. Facial asymmetry often reoccurs, necessitating secondary (orthognathic) surgery.

$\operatorname{Ref}(8,9,12,13,16,19,20,22,23)$

Level 2 Internal or external distractor for bilateral MDO (based on studies on patients with micrognathia)

Internal distractors are, compared to external distractors, associated with a significantly lower number of complications ( $22 \%$ to $33 \%$ versus $8 \%$ to $23 \%$; $\mathrm{p}=0.006-0.012$ )

External distractors may, compared to internal distractors, be associated with more relapse, a higher number of technical failures, and scarring of the skin.

More optimal vector control is seen when using external distractors, which may especially be needed in patients with severe mandibular hypoplasia.

$\operatorname{Ref}(31,32,36,44)$

Level 3

Complications of mandibular distraction osteogenesis (based on studies on patients with micrognatia)

The overall complication rate of mandibular distraction osteogenesis is $20 \%$ to $34 \%$, which is similar for patients with craniofacial microsomia $(24 \%-37 \%)$.

Minor, temporary complications occurred in 15\%-18\% of the patients and included hypoesthesia, local infections, pin loosening, dehiscence, or incorrect vector.

Moderate complications which required invasive treatment occurred in $4 \%-12 \%$ of the patients and included technical complications such as loosening of the device or pins.

Major, permanent complications occurred in 5\% - 8\% of the patients and included teeth damage, facial nerve palsy, TMJ ankylosis, non/malunion, hypertrophic scars, or a skeletal open bite.

Treatment with MDO at a younger age ( $<2$ years) may be associated with a lower risk of complications (mean follow up 2 years).

A higher distraction rate ( $>1 \mathrm{~mm} /$ day) is associated with a lower risk of complications compared to a conventional distraction rate of $1 \mathrm{~mm} /$ day, and is applied in significantly younger patients (10 months compared to 39 months).

$\operatorname{Ref}(21,23,36,42-44)$

\begin{tabular}{c}
\hline Level 3 Mandibular reconstruction with bone grafts \\
Mandibular reconstruction with non-vascularised bone \\
grafts is most commonly performed in craniofacial \\
microsomia patients with severe mandibular \\
hypoplasia (Pruzansky IIB - III). \\
Secondary surgery to restore the remaining or recurrence \\
of mandibular asymmetry after mandibular \\
reconstruction with costochondral grafts is needed in \\
18\% - 23\% of the patients. \\
Complications occur in 0-33\% of the patients with \\
craniofacial microsomia who had mandibular \\
reconstructions with bone grafts. \\
Complications of mandibular reconstruction in \\
craniofacial microsomia include graft infections, \\
postoperative hypoesthesia, (graft) ankylosis, \\
infection, malposition, or graft overgrowth. \\
Mandibular reconstruction with a free vascularised fibula \\
flap may be an option for patients with craniofacial \\
microsomia and severe mandibular hypoplasia who \\
have no other therapeutic options. \\
Ref $(9,19,24-26,33)$ \\
\hline
\end{tabular}




\begin{tabular}{ll}
\hline Level 3 & $\begin{array}{l}\text { Orthognathic surgery } \\
\text { Orthognatic surgery is often performed as primairy } \\
\text { treatment in patients with mild mandibular hypoplasia } \\
\text { or as secondary treatment in patients with severe } \\
\text { mandibular hypoplasia or bilateral craniofacial } \\
\text { microsomia. } \\
\text { Orthognathic surgery is performed to improve occlusal } \\
\text { canting and facial symmetry at skeletal maturity. } \\
\text { Ref }(9,10,19,24,27-29)\end{array}$ \\
\hline Level 3 & $\begin{array}{l}\text { Temporomandibular joint implants } \\
\text { Placement of custom-made TMJ implants at skeletal } \\
\text { maturity may be a good solution for patients with } \\
\text { failed autogenous mandibular reconstructions. } \\
\text { Improvement in occlusal relationship, mouth opening } \\
\text { and aesthetics without complications were seen in ten } \\
\text { studied patients with craniofacial microsomia (follow } \\
\text { up six months to four years). } \\
\text { Ref (35) }\end{array}$ \\
\end{tabular}

\section{Considerations}

- Overall quality of evidence

All conclusions that are based on literature that included patients with CFM are graded as level 3 evidence as all papers, besides two articles by Meazzini et al. $(11,12)$, are non-comparative studies. Conclusions on the type of distractors are based on large metaanalysis that showed consistent results and are based on comparative studies. This leads to a level 2 quality of evidence. Complications of mandibular distraction osteogenesis were also based on large meta-analysis, showing consistent results. Results are based on descriptive data and no comparison can be made. Therefore, this conclusion was graded as level 3 evidence.

\section{- Balance of benefits and harms}

The balance of benefits and harms is hard to assess due to the variance in indications for surgical treatment of mandibular/maxillary deformity in patients with CFM. In addition, the indication for surgical treatment may be strong in some cases, such as in patients with severe OSA, but less strong in other patients, such as patients with aesthetic concerns. Patients and their parents should be able to make a well-informed decision for surgical treatment. Therefore, it is important to inform the patients on the potential benefits and harms of treatment. This may include treatment of OSA, better occlusion or mouth opening leading to fewer or no feeding problems, better aesthetics, or fewer psychosocial problems. Using an internal distractor necessitates secondary surgery under general anaesthesia to remove the device, whereas the extraoral pins of an external distractor can be removed under local anaesthesia. Equally important is the need to inform patients on the risks of treatment, the burden of treatment, the risks of complications, and the need for potential additional surgeries in the future. In some cases, the benefits easily outbalance the potential harm. However, in other cases, such as in patients with aesthetic problems, the potential benefits and harms may be more difficult to assess. Patients and their parents should be well informed to optimise the process of shared decision making.

\section{- Outcome importance}

The importance of treatment for OSA is further delineated in Chapter 4.1 - Breathing problems. Occlusal problems or a limited mouth opening may lead to feeding problems. Besides, occlusal problems should be treated to minimise oral health damage. The severity of occlusal problems determines the need for and timing of treatment. Treatment of the mandibular/maxillary deformity to reduce psychosocial problems that are related to aesthetic concerns is based on the balance of benefits and harms. Treatment should only be considered after psychosocial support by a psychologist.

\section{- Costs and resources}

The impact on costs and resources of the given recommendations will vary per member state, depending on the available care providers and facilities. The recommendations concern the essential requirements for adequate treatment of patients with CFM and should thus be implemented. The use of custom-made TMJ implants is expensive. Additionally, developments in 3D planning could lead to an increase in costs. Costs are lowest and resources are most efficiently used when care for craniofacial disorders is centralised in a limited number of expert centres per member state. A general rule that can be applied is one expert centre per 10 million inhabitants.

\section{- Inequity of the recommendation}

The goal of the European Reference Networks (ERNs) is to eliminate inequality within Europe with regard to care for patients with rare diseases. At present, not every member state offers an expert centre for CFM, or the level of provided care does not (yet) meet all the requirements that are outlined in this guideline. By defining the baseline of required care for CFM, this guideline will help these member states to reach the appropriate level. The ERN on craniofacial anomalies and ENT disorders (ERN-CRANIO) can guide a patient in Europe to the available centres of expertise (www.ern-cranio.eu) and can support care providers with diagnosis and treatment advice.

\section{- Feasibility of the recommendation}

Recommendations refer to the general requirements for delivering optimal healthcare and are discussed with members from participating European countries. Quality of care was paramount in the discussions. Centralisation is proposed as one of the core values. However, in some countries the national organisation of healthcare might impede centralisation. National implementation of the ERNs that fits the situation of each country is necessary. For the member states with the lowest number of inhabitants, the establishment of a craniofacial centre might not be feasible, and collaboration with a craniofacial centre in the surrounding countries can be an option.

\section{- Acceptability of the recommendation}

It is expected that all stakeholders want to apply and will be applying the recommendations, because they are employed in ERNacknowledged institutions. National implementation plans are necessary to ensure that recommendations fit the situation in each country. In addition, not all countries participating in the ERNCRANIO are represented in the guideline development group, and new members will join within the coming years. For these countries, acceptance and implementation of ERN guidelines such as this guideline on CFM is obligatory.

\section{Rationale of the recommendations}

Treatment of mandibular/maxillary deformity may be indicated for various reasons, including obstructive sleep apnoea, occlusal problems, and aesthetics. The preferred treatment option is based on several factors, including the severity of mandibular hypoplasia, age of the patient, risk of complications, other optional treatment modalities, and so forth. There are various treatment options for correction of mandibular/maxillary deformity in patients with CFM. 
Nevertheless, based on the available literature, conclusions and recommendations can be made to improve care for patients with CFM. Recommendations on the optimal types of treatment for the previously reported indications for treatment (in Chapter 5.1.1.) were provided.

Obstructive sleep apnoea should be treated with non-surgical therapy if possible, to prevent the risks that are associated with surgery, which includes complications and a high burden for the patient. In patients with severe OSA, tracheostomy or mandibular distraction osteogenesis may be indicated. Primary mandibular distraction could prevent placement of a tracheostomy, but may not be suitable for all patients. Mandibular distraction could also be performed after placement of a tracheostomy for decannulation. The selection of a type of treatment is patient specific and should be based on multidisciplinary assessment. MDO may be indicated to end non-surgical treatment for sleep apnoea (e.g. CPAP), to allow decannulation, or to prevent tracheostomy. There is a difference in preference of timing of MDO. If possible, it is advised to perform mandibular distraction after the age of six because at this age, the first permanent teeth start to erupt and occlusal problems could be treated with a combined surgical and orthodontic approach. The benefits of early mandibular distraction should outweigh the need for additional surgery later in life, since Pluijmers et al. found that the total number of surgeries in patients with CFM is influenced by the age at which the first surgical intervention was performed (19).

Mandibular distraction osteogenesis could be performed in young, infant patients, which is associated with a lower risk of complications (44), but a bigger likelihood of repeat surgery (19). Additionally, a rapid distraction rate could be used in young patients under the age of twelve months, which is also associated with a lower risk of complications (36). Literature shows that the use of an internal distractor is preferable in most patients due to fewer relapses, lower complications rates, fewer technical failures and less scarring compared to an external distractor. However, secondary surgery under general anaesthesia is needed in patients with an internal distractor to remove the device. The use of an external distractor is preferred in patients with severe mandibular hypoplasia, when a high level of vector control is needed. Besides these aspects, the surgeon should be experienced with both treatment modalities.

Severe occlusal problems in patients with CFM could be treated with mandibular surgery/distraction during childhood. The orthodontist should be included in the team to optimise the treatment plan. If surgery can be postponed, it is advised to perform orthognathic surgery with or without mandibular distraction in patients who are skeletally mature to ensure stable results and to limit the number of procedures with the associated complication risks (27). Early surgery in patients with CFM to achieve facial symmetry is frequently debated in literature. Today, literature shows that CFM is a non-progressive disorder, meaning the facial and mandibular asymmetry in CFM does not increase over time (8). Psychosocial problems may be present due to aesthetic issues. Therefore, it is essential to start with psychosocial support during childhood. Surgical correction of the facial asymmetry with unilateral mandibular distraction or other treatment options could be helpful to prevent or treat psychosocial problems. However, the positive psychosocial effects of treatment should outweigh the risks of surgery and the risks of the need for additional surgeries (13). If surgical treatment is considered, the patients should be seen by a psychologist pre- and post-operatively to monitor expectation and acceptance. The risk for additional surgeries is important, since literature shows that most patients with CFM need additional surgery if they are treated at a young age (19). Therefore, it is advised to postpone mandibular/maxillary surgery for correction of facial asymmetry until skeletal maturity.
Mandibular reconstruction with a bone graft may be indicated in patients with severe mandibular hypoplasia and absence of the temporal mandibular joint. Various types of bone grafts have been reported in literature, but the costochondral graft is the most frequently used graft (19). It is advised to perform mandibular reconstruction with a costochondral graft during mid-dentition phase (after the age of six) to ensure good quality of the rib. In selected cases there might be an indication for free vascularised bone grafts. Reconstruction with a temporomandibular joint prosthesis shows promising results in a small group in a single centre and could be implanted in patients before or after skeletal maturity. This may be indicated in patients with failed previous mandibular reconstruction or (graft) ankylosis.

$3 \mathrm{D}$ planning is gaining in popularity and results in improved outcomes in orthognatic surgery (49). Therefore, it might help to improve planning and outcomes of mandibular/maxillary surgeries in patients with CFM. However, it requires facilities and imposes additional costs and might therefore not be applicable in all countries. The steering group considers access to 3D planning for treatment of the mandibular/maxillary deformity in patients with CFM as obligatory to ensure good quality of care.

\section{Recommendations}

\section{Obstructive sleep apnoea}

- Start with non-surgical treatment for the management (e.g. oxygen, CPAP) of mild-moderate OSA in infants with craniofacial microsomia. See Chapter 4.1 Breathing difficulties for recommendations.

- Perform a tracheostomy or mandibular distraction osteogenesis in infants with mandibular hypoplasia and severe OSA who do not respond to non-surgical treatment.

- If the aim of surgical treatment is to end non-surgical treatment (e.g. CPAP), perform elective mandibular distraction osteogenesis.

- Mandibular reconstruction with costochondral bone grafts should be performed after the age of six.

\section{Occlusal problems}

- For patients with craniofacial microsomia and severe occlusal problems, perform mandibular distraction osteogenesis in mixed dentition phase.

- A combined orthodontic and orthognathic surgery plan is mandatory to achieve and optimise stable longterm outcomes.

- Perform secondary orthognathic surgery to correct occlusion at skeletal maturity.

\section{Aesthetic problems}

- Postpone surgical correction of the mandibular/maxillary deformity for aesthetic reasons in patients with craniofacial microsomia until skeletal maturity.

- The implications of early surgery (i.e. repeat surgery) for psychosocial reasons should be discussed within the multidisciplinary team and with patient and caregivers.

- Psychological input is required pre- and post-operatively to monitor expectation and acceptance. 


\section{$3 D$ planning}

- Use 3D planning to optimise surgical outcome of mandibular and maxillary surgery in patients with CFM.

\section{Research gap}

Future studies on the ideal timing and long-term effects of mandibular distraction in patients with CFM could help determine an optimal treatment strategy. Breathing and feeding problems resulting from mandibular hypoplasia are probably more strongly related clinical issues in CFM than in other diagnoses. Studies on the optimal timing of MDO, choice for type of distractor, TMJ prosthesis, and safety of early MDO are needed. Multicentre prospective cohort studies with long-term outcome measures could offer insights into ideal timing and choice of treatment.

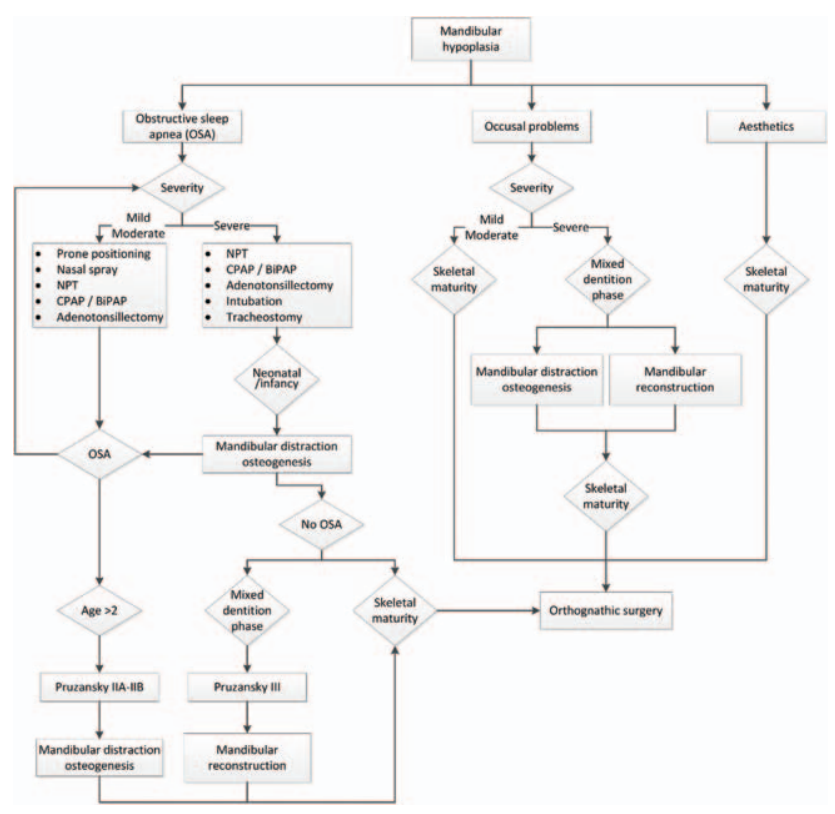

\section{References}

1. Heike CL, Wallace E, Speltz ML, Siebold B, Werler MM, Hing AV, et al. Characterizing facial features in individuals with craniofacial microsomia: A systematic approach for clinical research. Birth Defects Res A Clin Mol Teratol. 2016;106(11):915-26.

2. Caron C, Pluijmers BI, Wolvius EB, Looman CWN, Bulstrode $\mathrm{N}$, Evans RD, et al. Craniofacial and extracraniofacial anomalies in craniofacial microsomia: a multicenter study of 755 patients'. J Craniomaxillofac Surg. 2017;45(8):130210.

3. Kaban LB, Mulliken JB, Murray JE. Three-dimensional approach to analysis and treatment of hemifacial microsomia. Cleft Palate J. 1981;18(2):90-9.

4. Kaban LB, Moses MH, Mulliken JB. Correction of hemifacial microsomia in the growing child: a follow-up study. Cleft Palate J. 1986;23 Suppl 1:50-2.

5. S P. Not all dwarfed mandibles are alike. Birth defects: Original Article Series. 1969(5):120-9.
6. Grayson BH, Boral S, Eisig S, Kolber A, McCarthy JG. Unilateral craniofacial microsomia. Part I. Mandibular analysis. Am J Orthod. 1983;84(3):225-30.

7. Caron CJ, Pluijmers BI, Joosten KF, Mathijssen IM, van der Schroeff MP, Dunaway DJ, et al. Obstructive sleep apnoea in craniofacial microsomia: a systematic review. Int $\mathrm{J}$ Oral Maxillofac Surg. 2015;44(5):592-8.

8. Nagy K, Kuijpers-Jagtman AM, Mommaerts MY. No evidence for long-term effectiveness of early osteodistraction in hemifacial microsomia. Plast Reconstr Surg. 2009;124 (6):2061-71.

9. Pluijmers BI, Caron CJ, Dunaway DJ, Wolvius EB, Koudstaal MJ. Mandibular reconstruction in the growing patient with unilateral craniofacial microsomia: a systematic review. Int $\mathbf{J}$ Oral Maxillofac Surg. 2014;43(3):286-95.

10. van de Lande LS, Pluijmers BI, Caron C, Wolvius EB, Dunaway DJ, Koudstaal MJ, et al. Surgical correction of the midface in craniofacial microsomia. Part 1: A systematic review. J Craniomaxillofac Surg. 2018;46(9):1427-35.

11. Meazzini MC, Mazzoleni F, Bozzetti A, Brusati R. Does functional appliance treatment truly improve stability of mandibular vertical distraction osteogenesis in hemifacial microsomia? J Craniomaxillofac Surg. 2008;36(7):384-9.

12. Meazzini MC, Mazzoleni F, Bozzetti A, Brusati R. Comparison of mandibular vertical growth in hemifacial microsomia patients treated with early distraction or not treated: follow up till the completion of growth. J Craniomaxillofac Surg. 2012;40(2):105-11.

13. Ascenco AS, Balbinot P, Junior IM, D’Oro U, Busato L, da Silva Freitas R. Mandibular distraction in hemifacial microsomia is not a permanent treatment: a long-term evaluation. J Craniofac Surg. 2014;25(2):352-4.

14. Gui L, Zhang Z, Zang M, Liu W, Niu F, Yu B, et al. Restoration of facial symmetry in hemifacial microsomia with mandibular outer cortex bone grafting combined with distraction osteogenesis. Plast Reconstr Surg. 2011;127(5):1997-2004.

15. Kearns GJ, Padwa BL, Mulliken JB, Kaban LB. Progression of facial asymmetry in hemifacial microsomia. Plast Reconstr Surg. 2000;105(2):492-8.

16. Ko EW, Chen PK, Lo LJ. Comparison of the adult threedimensional craniofacial features of patients with unilateral craniofacial microsomia with and without early mandible distraction. Int J Oral Maxillofac Surg. 2017;46(7):811-8.

17. Caron C, Pluijmers BI, Maas B, Klazen YP, Katz ES, Abel F, et al. Obstructive sleep apnoea in craniofacial microsomia: analysis of 755 patients. Int J Oral Maxillofac Surg. 2017;46 (10):1330-7.

18. Lam DJ, Tabangin ME, Shikary TA, Uribe-Rivera A, Meinzen-Derr JK, de Alarcon A, et al. Outcomes of mandibular distraction osteogenesis in the treatment of severe micrognathia. JAMA Otolaryngol Head Neck Surg. 2014; 140(4):338-45.

19. Pluijmers BI, Caron C, van de Lande LS, Schaal S, Mathijssen IM, Wolvius EB, et al. Surgical Correction of Craniofacial Microsomia: Evaluation of Interventions in 565 Patients at Three Major Craniofacial Units. Plast Reconstr Surg. 2019; 143(5):1467-76.

20. Suh J, Choi TH, Baek SH, Kim JC, Kim S. Mandibular distraction in unilateral craniofacial microsomia: longitudinal results until the completion of growth. Plast Reconstr Surg. 2013;132(5):1244-52.

21. Wang X, Feng S, Tang X, Shi L, Yin L, Liu W, et al. Incidents of Mandibular Distraction Osteogenesis for Hemifacial Microsomia. Plast Reconstr Surg. 2018;142(4):1002-8. 
22. Weichman KE, Jacobs J, Patel P, Szpalski C, Shetye P, Grayson B, et al. Early Distraction for Mild to Moderate Unilateral Craniofacial Microsomia: Long-Term Follow-Up, Outcomes, and Recommendations. Plast Reconstr Surg. 2017;139(4):941e-53e.

23. Zhang RS, Lin LO, Hoppe IC, Swanson JW, Taylor JA, Bartlett SP. Early Mandibular Distraction in Craniofacial Microsomia and Need for Orthognathic Correction at Skeletal Maturity: A Comparative Long-Term Follow-Up Study. Plast Reconstr Surg. 2018;142(5):1285-93.

24. Bertin H, Mercier J, Cohen A, Giordanetto J, Cohen N, Lee $\mathrm{SH}$, et al. Surgical correction of mandibular hypoplasia in hemifacial microsomia: A retrospective study in 39 patients. J Craniomaxillofac Surg. 2017;45(6):1031-8.

25. Padwa BL, Mulliken JB, Maghen A, Kaban LB. Midfacial growth after costochondral graft construction of the mandibular ramus in hemifacial microsomia. J Oral Maxillofac Surg. 1998;56(2):122-7; discussion 7-8.

26. Tahiri Y, Chang CS, Tuin J, Paliga JT, Lowe KM, Taylor JA, et al. Costochondral grafting in craniofacial microsomia. Plast Reconstr Surg. 2015;135(2):530-41.

27. Fattah AY, Caro C, Khechoyan DY, Tompson B, Forrest CR, Phillips JH. Cephalometric outcomes of orthognathic surgery in hemifacial microsomia. J Craniofac Surg. 2014;25(5): 1734-9.

28. Yamaguchi K, Lonic D, Ko EW, Lo LJ. An integrated surgical protocol for adult patients with hemifacial microsomia: Methods and outcome. PLoS One. 2017;12(8):e0177223.

29. Pluijmers BI, van de Lande LS, Caron C, Wolvius EB, Dunaway DJ, Padwa BL, et al. Part 2: Is the maxillary canting and its surgical correction in patients with CFM correlated to the mandibular deformity? J Craniomaxillofac Surg. 2018;46(9):1436-40.

30. Kaban LB, Moses MH, Mulliken JB. Surgical correction of hemifacial microsomia in the growing child. Plast Reconstr Surg. 1988;82(1):9-19.

31. Rachmiel A, Aizenbud D, Eleftheriou S, Peled M, Laufer D. Extraoral vs. intraoral distraction osteogenesis in the treatment of hemifacial microsomia. Ann Plast Surg. 2000;45(4): 386-94.

32. Rachmiel A, Nseir S, Emodi O, Aizenbud D. External versus Internal Distraction Devices in Treatment of Obstructive Sleep Apnea in Craniofacial Anomalies. Plast Reconstr Surg Glob Open. 2014;2(7):e188.

33. Santamaria E, Morales C, Taylor JA, Hay A, Ortiz-Monasterio F. Mandibular microsurgical reconstruction in patients with hemifacial microsomia. Plast Reconstr Surg. 2008;122(6): 1839-49.

34. Liu H, Zhang X, Liu L, Chen Q, Shao J, Luo E. Combined Bimaxillary Distraction Osteogenesis Associated with Orthognathic Surgery for Hemifacial Microsomia in Adults. Aesthetic Plast Surg. 2017;41(3):650-60.

35. Polley JW, Girotto JA, Fahrenkopf MP, Dietze-Fiedler ML, Kelley JP, Taylor JC, et al. Salvage or Solution: Alloplastic Reconstruction in Hemifacial Microsomia. Cleft Palate Craniofac J. 2019;56(7):896-901.

36. Breik O, Tivey D, Umapathysivam K, Anderson P. Does the Rate of Distraction or Type of Distractor Affect the Outcome of Mandibular Distraction in Children With Micrognathia? J Oral Maxillofac Surg. 2016;74(7):1441-53.

37. Breik O, Tivey D, Umapathysivam K, Anderson P. Mandibular distraction osteogenesis for the management of upper airway obstruction in children with micrognathia: a systematic review. Int J Oral Maxillofac Surg. 2016;45(6):769-82.
38. Breik O, Umapathysivam K, Tivey D, Anderson P. Feeding and reflux in children after mandibular distraction osteogenesis for micrognathia: A systematic review. Int $\mathrm{J}$ Pediatr Otorhinolaryngol. 2016;85:128-35.

39. Ow AT, Cheung LK. Meta-analysis of mandibular distraction osteogenesis: clinical applications and functional outcomes. Plast Reconstr Surg. 2008;121(3):54e-69e.

40. Paes EC, Mink van der Molen AB, Muradin MS, Speleman L, Sloot F, Kon M, et al. A systematic review on the outcome of mandibular distraction osteogenesis in infants suffering Robin sequence. Clin Oral Investig. 2013;17(8):1807-20.

41. Tsui WK, Yang Y, Cheung LK, Leung YY. Distraction osteogenesis as a treatment of obstructive sleep apnea syndrome: A systematic review. Medicine (Baltimore). 2016;95(36):e4674.

42. Verlinden CR, van de Vijfeijken SE, Jansma EP, Becking AG, Swennen GR. Complications of mandibular distraction osteogenesis for congenital deformities: a systematic review of the literature and proposal of a new classification for complications. Int J Oral Maxillofac Surg. 2015;44(1):37-43.

43. Master DL, Hanson PR, Gosain AK. Complications of mandibular distraction osteogenesis. J Craniofac Surg. 2010;21(5):1565-70.

44. Tahiri Y, Viezel-Mathieu A, Aldekhayel S, Lee J, Gilardino M. The effectiveness of mandibular distraction in improving airway obstruction in the pediatric population. Plast Reconstr Surg. 2014;133(3):352e-9e.

45. Caron C, Pluijmers BI, Joosten KFM, Dunaway D, Padwa BL, Wolvius EB, et al. Feeding difficulties in craniofacial microsomia: A multicenter retrospective analysis of 755 patients. J Craniomaxillofac Surg. 2018;46(10):1777-82.

46. Caron CJ, Pluijmers BI, Joosten KF, Mathijssen IM, van der Schroeff MP, Dunaway DJ, et al. Feeding difficulties in craniofacial microsomia: a systematic review. Int $\mathrm{J}$ Oral Maxillofac Surg. 2015;44(6):732-7.

47. Dion K B, E. Physical attractiveness and peer perception among children. Sociometry. 1974;37(1):1-12.

48. Mofid MM, Manson PN, Robertson BC, Tufaro AP, Elias JJ, Vander Kolk CA. Craniofacial distraction osteogenesis: a review of 3278 cases. Plast Reconstr Surg. 2001;108(5):110314; discussion 15-7.

49. Hsu SS, Gateno J, Bell RB, Hirsch DL, Markiewicz MR, Teichgraeber JF, et al. Accuracy of a computer-aided surgical simulation protocol for orthognathic surgery: a prospective multicenter study. J Oral Maxillofac Surg. 2013;71(1):128-42.

\section{CHAPTER 5. SURGICAL TREATMENT}

\subsection{Facial nerve}

\section{Introduction}

Palsy of the facial nerve can be seen in patients with craniofacial microsomia (CFM). The prevalence of facial palsy in patients with CFM is $22 \%-53 \%$ and may be unilateral or bilateral (1-4). In the O.M.E.N.S. classification, which is commonly used to grade the severity of patients with CFM, the Nerve score categorises the loss of function of the upper (N1), lower (N2), or total facial nerve (N3) (5).

After exiting the brain stem, the facial nerve progresses through the skull and branches to the chorda tympani, posterior auricular nerve and to the digastric and stylohyoid muscles. The main branches of the facial nerve originate in the parotid and include the frontal, zygomatic, buccal, marginal mandibular, and cervical 
branch. Palsy of the facial nerve, which is due to congenital underdevelopment in patients with CFM, may cause problems with eye closure, articulation of speech, oral continence, or asymmetric facial mimics and smile (6).

Since a considerable number of patients with CFM experience facial palsy, recommendations on the indications for treatment and the most optimal treatment modalities are needed. Therefore, the following questions were posed:

5.2.1 What is the indication for surgical treatment of facial nerve anomaly in patients with craniofacial microsomia? 5.2.2 What is the most optimal treatment modality for facial nerve anomaly in patients with craniofacial microsomia related to functional deficits and aesthetics?

The answers to these questions are important to patients since various functional and aesthetic problems may occur due to facial palsy. For example, palsy of the upper branches of the facial nerve can lead to an inability to close the eye (lagophthalmos). Awareness of the consequences of lagophthalmos is important, since corneal exposure can lead to keratitis, ulceration, and eventually endophthalmitis which can cause blindness. Palsy of the lower eyelid may also lead to ptosis and lagophthalmos.

Besides insufficient eye closure, ptosis of the eyebrow due to paralysis of the frontalis muscle may also be seen in patients with facial palsy. Since ptosis may lead to vision loss, surgical treatment may be indicated. In addition, facial palsy may also cause oral related problems, such as drooling, dysphagia, or speech- or feeding problems, due to lack of lower lip support and oral sphincteric incompetence (7).

Inadequate functioning of facial mimics can lead to social problems and psychological stress (8). Although patients with CFM commonly experience more facial differences besides facial palsy, the impact on psychosocial functioning may be significant.

\section{Literature search}

A systematic search of literature was performed to identify all available literature on CFM and synonyms. The search was conducted in Embase, Pubmed/Medline Ovid. The full search strategy is reported in the supplementary material.

Inclusion and exclusion criteria:

Type of studies - Original articles

- Systematic review of sufficient quality:

- The question in the systematic review

matches the question of the guideline.

- The search of the systematic review was conducted in at least two relevant databases, such as the Cochrane Library, Medline/Pubmed.

- The full search strategy was reported.

- No relevant items were missing in the search strategy.

Type of patients - Patients with craniofacial microsomia

- Patients with facial palsy

Subject

- Facial palsy

Exclusion criteria

- Original studies with $<10$ included patients

- Articles published before 1980

- Case reports

- Expert opinion

- Letters

- Editorials

Literature was screened on title and abstract. No articles on the indication or outcomes of treatment of facial nerve deformity in patients with CFM were available. Therefore, the Dutch Guideline on peripheral facial palsy was included in this chapter (9). Since this guideline was published in 2009 and included literature until 2007, an additional search, based on the search strategy used in the Dutch Guideline, was performed. The full search strategy is shown in the appendix. All literature on indication and/or treatment outcomes of facial palsy from 2007 to the present was included. A total of 888 articles was screened on title and abstract. Since the quality of evidence of the identified literature was considered to be low, narrative reviews were also included. Besides the Dutch Guideline on peripheral facial palsy, 27 other articles were included: two systematic reviews/meta-analyses by Luijmes et al. (10) and Bos et al. (11); eleven retrospective-cohort studies by Hontanilla et al. (12), Loyo et al. (13), Terzis et al. (2010) (14), Chen et al. (15), Lindsay et al. (16), Roy et al. (7), Chuang et al. (17), Gousheh et al. (18), Harrison et al. (19), Hontanilla et al. (20), and MacQuillan et al. (21); seven case series by Baheerathan et al. (22), Banks et al. (23), Bianchi et al. (24), Panossian et al. (25), Terzis et al.(2009) (26), and Terzis et al. (2009) (27); and seven narrative reviews by Heike et al. (5), Reddy et al. (6), Volk et al. (28), Rahman et al. (29), Majid et al. (30), Barr et al. (31), and Terzis et al. (2008) (32).

5.2.1 What is the indication for surgical treatment of facial nerve anomaly in patients with craniofacial microsomia?

The literature search for this question was directed towards more specific research questions related to clinical problems, symptoms, treatment options, consequences of not treating, and requirements for treating facial nerve anomalies (Appendix 1, http://links.lww.$\mathrm{com} / \mathrm{SCS} / \mathrm{B} 697)$. Unfortunately, no literature on the indication for treatment of lagophthalmos in patients with CFM was found. Therefore, the Dutch Guideline on peripheral facial palsy was included in this question.

Review of literature

The Dutch guideline on peripheral facial palsy advises to start lubrication of the eye if the patient has complaints of the eye (dryness, irritation, tears, photophobia), difficulties with full closure of the eyelid, a positive Bell's phenomenon, or irritation of the conjunctiva. Surgical treatment of the eyelid is, according to the Dutch guideline, indicated if conservative treatment (lubrication) is no longer sufficient. To prevent blindness, eyelid surgery is indicated in patients with a corneal ulcer (9). Surgical treatment of facial palsy in patients with CFM may also be indicated to restore facial animations and improve aesthetics. Various techniques have been described in literature, static or dynamic, to restore the smile in patients with facial palsy. However, no literature on the indication for surgical treatment to restore facial function, eyebrow ptosis or oral incompetence was found.

Birgfeld and Heike state that the timing of facial reanimation surgery in patients with CFM should be based on patient's preferences and other surgical interventions. Microtia reconstruction and major craniofacial or orthognathic surgery should ideally be performed prior to facial reanimation surgery (5).

In their systematic review on the quality of life in patients with peripheral facial palsy, Luijmes et al. found that patients had a significant increase of the quality of life after (surgical) treatment of their facial palsy (mean FaCE score 43.38; 95\% CI: $40.48-$ 46.27; $\mathrm{p}<0.001)(10)$. Quality of life was measured using various questionnaires and various treatment modalities, such as botulinum toxin injections, free flaps, or static procedures, were included. It should be noted that patients with CFM have multiple, often complex, facial deformities, making comparison of the outcomes of treatment on the quality of life of other patient groups difficult. 


\section{Conclusions}

\begin{tabular}{l} 
Level 4 Indication \\
$\begin{array}{l}\text { Surgical treatment of the upper or lower eyelid in patients } \\
\text { with facial palsy to protect against prolonged corneal } \\
\text { exposure is indicated if conservative treatment is no } \\
\text { longer sufficient. } \\
\text { Surgical treatment of the upper or lower eyelid in patients } \\
\text { with facial palsy is indicated in patients with a corneal } \\
\text { ulcer. } \\
\text { Ref (9) }\end{array}$ \\
\hline
\end{tabular}

\begin{tabular}{l} 
Level $4 \quad \begin{array}{l}\text { Indication } \\
\text { The timing of facial reanimation surgery should be based } \\
\text { on patients' preferences and other surgical } \\
\text { interventions. Microtia reconstruction and major } \\
\text { craniofacial or orthognathic surgery should ideally be } \\
\text { performed prior to facial reanimation surgery. } \\
\operatorname{Ref}(5)\end{array}$ \\
\hline
\end{tabular}

\section{- Outcome importance}

A considerable number of patients with CFM experience facial palsy. If functional problems occur, treatment is essential to prevent further harm such as blindness. In case of aesthetic problems, treatment may still be of great importance to patients. Treatment should be considered after psychosocial support by a psychologist.

\section{- Costs and resources}

The impact on costs and resources of the given recommendations will vary per member state, depending on the available care providers and facilities. The recommendations concern the essential requirements for adequate treatment of patients with CFM and should thus be implemented. Costs are lowest and resources are most efficiently used when care for craniofacial disorders is centralised in a limited number of expert centres per member state. A general rule that can be applied is one expert centre per 10 million inhabitants.

\section{- Inequity of the recommendation}

The goal of the European Reference Networks (ERNs) is to eliminate inequality within Europe with regard to care for patients with rare diseases. At present, not every member state offers an expert centre for CFM, or the level of provided care does not (yet) meet all the requirements that are outlined in this guideline. By defining the baseline of required care for CFM, this guideline will help these member states to reach the appropriate level. The ERN on craniofacial anomalies and ENT disorders (ERN-CRANIO) can guide a patient in Europe to the available centres of expertise (www.ern-cranio.eu) and can support care providers with diagnosis and treatment advice.

\section{Considerations \\ - Overall quality of evidence}

Literature on the indications for treatment of facial nerve problems in patients with CFM is very limited. Therefore, indirect literature on facial palsy in general was also included. The Dutch guideline on peripheral facial palsy advised treatment in case of corneal exposure. Since this was based on agreement among the guideline committee, the level of evidence was graded on level 4. The advice of Birgfeld and Heike to wait to perform facial reanimation surgery after other major surgery was also based on expert opinion and therefore graded on level 4 (5). The conclusion on quality of life was based on a meta-analysis of prospective and retrospective cohort studies and case series. The quality of evidence is graded on level 1, since it was a well-designed meta-analysis of fourteen articles that used different predetermined questionnaires and the results were all positive for quality of live.

\section{- Balance of benefits and harms}

Since the prevalence of facial palsy in patients with CFM is high (22\%-53\%) and can have functional consequences, treatment may be indicated. Especially for problems with eye closure, which may result in a corneal ulcer, endophthalmitis, and eventually blindness, it is essential to treat timely. In case of aesthetic problems, the indication for treatment may be less strong, but nevertheless some patients may experience negative psychosocial consequences that may make treatment necessary. Since patients with CFM do often have additional facial deformities besides the facial nerve deficits, psychosocial support and treatment by a psychologist is of major importance. If the indication for treatment of the facial nerve deformity is purely aesthetic, the potential complications of surgery (such as hematomas, abscesses, flap failure) are important to keep in mind. The patients should be well informed about the risks of treatment to make a well-considered decision.

\section{- Feasibility of the recommendation}

Recommendations refer to the general requirements for delivering optimal healthcare and are discussed with members from participating European countries. Quality of care was paramount in the discussions. Centralisation is proposed as one of the core values. However, in some countries the national organisation of healthcare might impede centralisation. National implementation of the ERNs that fits the situation of each country is necessary. For the member states with the lowest number of inhabitants, the establishment of a craniofacial centre might not be feasible, and collaboration with a craniofacial centre in the surrounding countries can be an option.

\section{- Acceptability of the recommendation}

It is expected that all stakeholders want to apply and will be applying the recommendations, because they are employed in ERNacknowledged institutions. National implementation plans are necessary to ensure that recommendations fit the situation in each country. In addition, not all countries participating in the ERNCRANIO are represented in the guideline development group, and new members will join within the coming years. For these countries, acceptance and implementation of ERN guidelines such as this guideline on CFM is obligatory.

\section{Rationale of the recommendations}

The indications for treatment of facial nerve palsy vary from functional to aesthetic. In patients unable to achieve full closure of the eye treatment with lubricants is indicated, although the cause of problems is not treated. Surgical interventions should be considered in these patients. Other functional problems, such as eyebrow ptosis or oral incompetence may also occur in patients with facial palsy and should be treated to improve the quality of life. Referral to an ophthalmologist is needed in all patients with lagophthalmos. 
Aesthetic problems are considered to be another reason to treat facial nerve palsy in patients with CFM, although no literature on this topic is available. The involvement of a psychologist is essential during childhood for patients with CFM to learn to cope with their facial differences. The ICHOM Standard Set for Craniofacial Microsomia advises to assess smile and facial movement by using the CleftQ appearance questionnaire at age 8, 12, and 22. Treatment of facial nerve palsy for aesthetic reasons should be based on wellinformed patient preferences and be integrated with other planned surgeries.

\section{Recommendations}

\section{Indications for treatment}

- Provide all patients with craniofacial microsomia with psychosocial support.

- Refer all craniofacial microsomia patients with lagophthalmos to an ophthalmologist.

- Surgical treatment of the upper or lower eyelids should be considered in patients with craniofacial microsomia and loss of function of the upper facial nerve branches.

- Coordinate the timing of facial reanimation surgery in patients with craniofacial microsomia in the planning of other major surgeries.

- Facial movement should by assessed with the CleftQ Appearance at age 8, 12, and 22 .

\section{Research gap}

No literature on the indication for treatment of facial palsy in CFM was available. Literature on the (psychological) consequences of facial palsy in CFM could help determine the indication for treatment.

\subsubsection{What is the most optimal treatment modality for facial} nerve anomaly in patients with craniofacial microsomia related to functional deficits and aesthetics?

The literature search for this question was directed towards more specific research questions related to treatment options, complications, advantages and disadvantages, short and long term results and treatment burden for treating facial nerve anomalies (Appendix 1, http://links.lww.com/SCS/B697). Patient outcomes that are relevant for this chapter are functional deficits and aesthetics. This review of literature addresses the most optimal treatment modalities related to the functional and aesthetic consequences of facial palsy. The functional problems and their treatment modalities in this chapter are subdivided into problems with eye closure, oral problems, and dynamic facial reanimation.

\section{Review of literature Eye}

Various treatment modalities are available to treat the inability to close the eye in patients with facial palsy. The guideline on peripheral facial palsy advises to start conservative treatment using lubrication in all patients with facial palsy and complaints of the eye. In patients with CFM, the facial palsy is congenital and more permanent solutions should be striven for. The goal of surgery of the upper eyelid is to improve vision, prevent corneal erosion and maintain or improve aesthetics (20).

The upper eyelid can be lowered using botulinum toxin, muscle transfers, or placing a gold weight. Injection of botulin toxin has been found to be effective by establishing a ptosis by temporarily paralysing the levator palpebrae superioris muscle (28). The duration of the effect is approximately three months and there is a chance that therapy is unsuccessful due to injecting too little or too much botulinum toxin (9).

Lengthening of the levator palpebrae superioris muscle by aponeurosis interposition could be performed to achieve full eye closure, although this technique is less frequently described in literature. The technique is complex compared to botulinum toxin or placement of a gold weight, but may be have a better cosmetic outcome compared to gold weights (9).

Placement of a gold weight in the upper eyelid is a popular technique to correct lagophthalmos. A predetermined gold weight is implanted in the eyelid that supports closure of the eye. Other materials such as a platinum chain are currently used too (13). Although placement of gold weights is reported to be the preferred method for several surgeons due to the predictable results, good aesthetic outcome, reversibility, relatively easy technique the fact that and it can be placed under local anaesthesia in adults, various complications may occur (20). This includes migration or extrusion of the gold weight, soreness, or persistent lagophthalmos (22). The incidence of extrusion of the gold weight was $2.6 \%$ in 2,000 studied patients, which may be prevented by attaching the gold weight on the tarsal plate using sutures $(22,29)$. In a case series by Baheerathan that described the experience with gold weights in 16 patients, one patient reported extrusion of the gold weight after five months and one patient needed a heavier gold weight due to residual lagophthalmos (22). No infections, implant migration, visual problems or keratopathy occurred. All patients had adequate eyelid closure at the last follow-up and fifteen of the sixteen patients were satisfied with the outcome (22). Surgical revision after placement of a upper eyelid weight was needed in 18 of the 95 patients (19\%) studied by Loyo et al. (13). One patient had implant extrusion, two patients bulging/superficial implants, two patients implant migration, five patients had persistent lagophthalmos, and nine patients eyelid ptosis. In a retrospective cohort study by Hontanilla et al. 58 patients had gold weights implanted (20). All patients had complaints of dry eyes. Five patients $(8.6 \%)$ had extrusion of the gold weight, which was due to material intolerance in two patients and exchanged for a different weight in the other three patients without complications. The complaints of eye dryness improved in $90.7 \%$ of the patients $(n=49)$. Corneal ulcers, which were present in 14 patients, resolved without further treatment in $85.7 \%$ of the patients $(n=12)(20)$. It is important to note that all these studies included adult patients only and no literature on children is available.

Facial palsy may also lead to laxity or ectropion of the lower eyelid which disrupts physiological tear drainage $(20,28)$. This inability to achieve full closure of the eye may again lead to keratitis or corneal erosion. The aim of surgery of the lower eyelid is to improve tear drainage and correct the ectropion or ptosis (20). Tarsorrhaphy has been the golden standard to achieve this, but other techniques such as lateral tarsal suspension or the use of a fascia lata strip, which also provide corneal coverage but produce better aesthetic appearance, have replaced this (20). In addition, tarsorrhaphy may cause loss of peripheral vision and its use is therefore discouraged (29). In the retrospective study by Loyo et al. lower eyelid surgery was performed in $47 \%$ of the patients with paralytic lagophthalmos (47/101 patients) (13). All patients had procedures that addressed the lateral canthal tendon and 10 of the 47 patients underwent medial canthopexy. Of these patients, 29\% (14/47 patients) required additional lower eyelid procedures. Hontanilla et al. retrospectively studied 43 patients with lower eyelid suspension with a tendon sling (20). Successful correction of the ectropion/ ptosis and epiphora was achieved in respectively $93.9 \%$ and $91.9 \%$. Eight patients $(18.6 \%)$ needed additional surgery to increase tendon tension. No complications that required surgical intervention occurred. The authors discourage the use of a dynamic technique 
to reanimate the lower eyelid since there is little movement of the lower eyelid during physiological closure of the eye.

Terzis et al. studied paediatric patients who underwent dynamic reanimation procedures to restore the blink reflex (14). A total of 42 patients underwent nerve transfers such as cross-facial nerve grafting or minihypoglossal nerve transfers, and 14 patients underwent eye sphincter substitution techniques, using the temporalis, platysma, frontalis, or pectoralis minor muscle. A statistically significant improvement in blink scores, calculated as the reduction of the palpebral fissure during blinking, was seen in all patients who underwent dynamic procedures $(\mathrm{p}=<0.05)$. Improvement in blinking was especially seen in patients who underwent direct orbicularis oculi muscle neurotisation. Regarding the various types of muscle transfers, the frontalis transfer was found to achieve the highest level of improvement in blinking. Complications occurred in a single patient, who had a severe postoperative infection in a pedicled frontalis, which was successfully treated.

Besides difficulties in eye closure, brow ptosis may also lead to visual problems or cosmetic concerns. A brow lift was performed in 47 of the 101 patients studied by Loyo et al.(13). Revision of surgery was needed in $17 \%$ of the patients $(8 / 47)$, which included a direct brow lift ( $5 / 8$ patients), temporal brow lift ( $2 / 8$ patients), and pretrichial approach (1/8 patients).

\section{Oral problems}

Facial palsy may also cause problems in oral competence, speech, or feeding due to weakened lower lip support. Asymmetry caused by the labial depressors of the face can be restored by injecting botulinum toxin or by myomectomy of the contralateral depressor labii inferioris. Injection of botulinum toxin in the contralateral depressor labii inferioris can be used as a temporary approach to evaluate the effect which can be followed by myomectomy of the muscle. Chen et al. found good to fair results of dynamic balance after myomectomy and injections with botulinum toxin in their studied cohort of 76 patients (15). No significant differences in posttreatment results between the two groups were seen. Although no complications occurred, a recurrence rate of $24 \%$ was seen in the patients who had surgical myomectomy. This was further treated with botulinum toxin injections.

A different, potentially superior approach is to restore lip depression with dynamic techniques. This includes transposition of the ipsilateral anterior belly of the digastric muscle or the use of a fascia lata sling to the ipsilateral platysma muscle $(6,28,31)$. Interestingly, the anterior belly of the digastric muscle may be underdeveloped in $40 \%$ of the patients with CFM, and in $55 \%$ of the patients with CFM and marginal mandibular nerve palsy (21). Therefore, imaging to check the presence of the digastric muscle should be performed before lower lip reanimation surgery starts (21). Lindsay et al. retrospectively studied the outcomes of treatment of an asymmetric lower lip and proposed a treatment algorithm (16). A total of 58 patients were included. Of the 57 patients treated with botulinum toxin injections in the contralateral depressor labii inferioris, 48 were satisfied with the result. No poor aesthetic outcome, oral incompetence, or speech problems were seen after injection with botulinum toxin. Four patients were treated with a digastric muscle transfer; three were satisfied with the result and one patient needed additional debulking. The authors' proposed treatment algorithm starts with injecting lidocaine into the contralateral depressor labii inferioris. If this results in improvement, injection of botulinum toxin could be offered. This can be repeated every four to six months and after two to three treatments a more definitive treatment by performing myomectomy of the depressor labii inferioris can be considered. If the botulinum toxin injections do not lead to satisfactory results, alternative treatments such as digastric muscle transfer should be considered (16).

\section{Dynamic facial reanimation}

Dynamic surgical techniques can be used in children with congenital facial palsy for facial reanimation. The aim of surgery is to achieve a symmetric face at rest and reanimate a spontaneous, symmetric smile (18). Cross-facial nerve grafting with a gracilis transfer is considered to be the most favourable option in literature, although the use of other nerves or of local regional flaps can also be considered $(6,31)$. The sural nerve is often used as a graft and attached to a cranial nerve for the procedure of cross-facial nerve grafting. The selection of the nerve transfer is based on patientspecific characteristics, such as the type of palsy (uni- or bilateral, complete or incomplete), the availability of the preferred or neighbouring nerve(s), or the rehabilitation needs $(23,32)$. The contralateral facial nerve is considered to be the most favourable nerve, due to its ability to induce spontaneous contraction of the innervated muscle and thereby create a natural smile $(9,11,32)$. If the contralateral facial nerve can't be used to innervate the free muscle on the paralytic side, alternative motor nerves such as the masseter, hypoglossal and accessory nerves can be used (32). For rare cases, other motor nerves such as the ipsilateral $\mathrm{C} 7$ root or the cervical plexus can be used for facial reanimation (32).

Although the contralateral facial nerve is considered to be the most favourable nerve transfer, the masseter nerve is also frequently used, especially in patients with bilateral facial palsy due to its direct connection and relatively good outcomes $(7,12,32)$. In a case series by Bianchi et al. the aesthetic results of masseter nerve transfer were considered to be moderate in $17 \%$, good in $51 \%$, and excellent in $32 \%$ of the patients (24). Major complications not seen and minor complications included hematomas, abscesses, or postoperative thrombosis $(7,18,23,24)$. In patients with a masseter nerve transposition compared to cross-facial nerve graft, movement was found to be more symmetrical, and displacement and contraction velocity of the commissure were significantly greater $(11,12)$. However, spontaneous movement of the innervated free flap after masseter nerve transfer is hard to achieve and the rates of a spontaneous smile are $0 \%$ in some studies, although other studies report higher rates of $55-100 \%(11,12,17,18)$. In addition, outcomes of masseter nerve transfer were found to be more successful ( $>2 \mathrm{~mm}$ change in smile excursion) in patients with flaccid facial paralysis ( $88 \%$ success) compared to patients with postparalysis facial palsy ( $20 \%$ success) $(23)$. The masseter nerve transfer is considered to be useful in patients with bilateral facial palsy, patients with limited facial movement on the healthy side, patients with major asymmetry at rest, or patients who want to avoid a two-stage procedure $(11,23,24,32)$.

Six to twelve months after this first nerve grafting procedure, the free muscle is harvested and transferred to the donor site $(6,31)$. The gracilis muscle is frequently preferred because of the low donor-site morbidity, re-innervation potential, reliability of the flap, and ease of dissection $(6,31)$. Other donor flaps such as the latissimus dorsi or pectoralis minor have also been described in literature (18). Harrison et al. described outcomes of 528 patients who were treated with a pectoralis minor muscle transfer and 28 patients with a latissimus dorsi transfer (19). Results were excellent in $63 \%$, good in $28 \%$, poor in $5 \%$, and no improvement was seen in $3 \%$ of the patients (19). The authors prefer the use of the pectoralis minor because of its size and shape (19).

Complications after dynamic facial reanimation are not very common. Gousheh et al. reported complications in twelve of the 655 patients (2\%). All complications were minor and all grafts survived (18). Chuang et al. reported failure of four gracilis muscles in 362 
patients $(1 \%)$, which were successfully replaced in all cases (17). The long-term outcomes of free-muscle transfer were studied by Terzis et al. $(26,27)$. A total of 32 children with a follow-up of at least five years were included (26). Almost all patients (91\%) needed additional surgical revisions, which included debulking, defatting, reanchoring the muscle graft, or a mini-temporalis transfer to the oral commissure. All flaps survived and all patients showed improved function and symmetry at two-year follow-up $(\mathrm{p}=<0.001)$. This improvement remained over time. Muscle function and aesthetic outcomes showed further improvement over time $(\mathrm{p}=<0.001)$. These results were also seen in a different study by the same authors that addressed the long-term outcomes of freemuscle transfers in adults (27). The authors conclude that these data support the use of free-muscle transfer in children and the technique is effective to restore a coordinated functional and symmetric smile $(26,27)$.

The use of regional flaps can be considered in patients in whom a cross-facial nerve graft or free-muscle transfers is not an option (18). Regional flaps cause shorter hospital stay and operative times, and limited donor-side morbidity compared to cross-facial nerve grafting (25). Regional temporalis or masseter flaps are less commonly used in children due to the bulky aspect of the masseter, temporal hollowing, and the inability to respond to emotions and form a natural smile $(28,31)$. Although no spontaneous smile can be achieved, commissure displacement after lengthening temporalis myoplasty was found to be $8.2 \mathrm{~mm}$ (range $5-13 \mathrm{~mm}$ ) in the 14 patients studied by Panossian et al. (25).

\section{Conclusions}

Level 3 Eye
Lowering of the upper eyelid to prevent keratitis or
corneal erosion can be achieved by injection of
botulinum toxin, lengthening of the levator palpebrae
superioris muscle or placement of a gold weight in the
upper eyelid.
Placement of a gold or platinum weight to correct
lagophthalmos is a preferred method due to good
aesthetic outcome, predictable results, reversibility,
easy technique, and the fact that it can be placed under
local anaesthesia in adults.
Surgical revision after placement of a gold weight is
needed in up to 19\% of patients, due to extrusion or
migration of the implant, persistent lagophthalmos, or
ptosis.
Ref $(9,13,20,22,29)$

\section{Level 3}

Eye

Eye sphincter substitution techniques by using muscle transfers or cross-facial nerve grafting can lead to a significant improvement in blinking $(\mathrm{p}=<0.05)$.

The highest level of improvement in blinking is seen after transfer of the frontalis muscle compared to the use of the platysma, frontalis, or pectoralis minor muscle.

Surgical correction of ptosis of the eyebrow in patients with facial palsy may be indicated to treat visual or cosmetic problems.

$\operatorname{Ref}(13,14)$

\begin{tabular}{|c|c|}
\hline Level 3 & $\begin{array}{l}\text { Oral } \\
\text { Symmetric lip depression may be restored by repeated } \\
\text { injections of botulinum toxin or myomectomy of the } \\
\text { non-affected depressor labii inferioris, or by dynamic } \\
\text { reconstruction. } \\
\text { Results after botulinum toxin injections or myomectomy } \\
\text { of the non-affected depressor labii inferioris are } \\
\text { considered to be fair to good and no significant } \\
\text { difference in post treatment results of the two } \\
\text { treatment options are seen. } \\
\text { Dynamic reconstruction of lower lip depression includes } \\
\text { transposition of the ipsilateral anterior belly of the } \\
\text { digastric muscle or the use of a fascia lata sling to the } \\
\text { ipsilateral platysma muscle. } \\
\text { Due to the high prevalence of agenesis of the anterior } \\
\text { belly of the digastric muscle in patients with } \\
\text { craniofacial microsomia (55\%), imaging of the muscle } \\
\text { should be performed before reanimation surgery of the } \\
\text { lower lip is undertaken. } \\
\text { Ref }(6,9,15,16,21,28,31)\end{array}$ \\
\hline
\end{tabular}

Level 4

$\underline{\text { Oral }}$

Proposed treatment algorithm for the asymmetric lower lip:

- Start with injection of botulinum toxin in contralateral depressor

- Repeat treatment if results are satisfactory

- After 2-3 treatments and good results, myomectomy can be considered

- No satisfactory results: consider digastric muscle transfer

$\operatorname{Ref}(16)$

\begin{tabular}{c}
\hline Level 4 Dynamic facial reconstruction / aesthetics \\
To restore facial animation and a spontaneous smile, \\
cross-facial nerve grafting is considered to be the \\
preferred treatment for unilateral craniofacial \\
microsomia. \\
Other motor nerves, such as the masseter, hypoglossal, \\
accessory or cervical nerves can be used if cross-facial \\
nerve grafting is not possible. \\
The gracilis muscle is often preferred due to the low \\
donor-site morbidity, re-innervation potential, \\
reliability of the flap, and ease of dissection. Other free \\
flaps, such as the latissimus dorsi or pectoralis minor \\
could also be used. \\
$\operatorname{Ref}(6,9,11,18,19,23,31,32)$
\end{tabular}

Correction of the lower eyelid can be achieved by tendon sling, lateral tarsal suspension, or by a fascia lata strip. Successful correction can be achieved in $>90 \%$ of patients.

The use of tarsorrhaphy is discouraged due to poor cosmetic outcome and risk of peripheral vision loss.

The rate of surgical revisions after a tendon sling or medial canthopexy to correct paralytic lagophthalmos varies from 18 to $29 \%$.

$\operatorname{Ref}(9,13,20,29)$

$\operatorname{Ref}(6,9,11,18,19,23,31,32)$ 


\begin{tabular}{c}
\hline Devel $3 \quad$ Dynamic facial reconstruction / aesthetics \\
Regional flaps, such as temporalis muscle transfers, can \\
be considered for dynamic facial reanimation if cross- \\
facial nerve grafting or free-muscle transfers are not \\
possible. \\
The use of regional flaps leads to shorter hospital stay and \\
operative times and limited donor-side morbidity \\
compared to free flaps. But no spontaneous smile can \\
be achieved and temporal hollowing may occur. \\
$\operatorname{Ref}(18,25,28,31)$ \\
\hline
\end{tabular}

\begin{tabular}{l}
\hline Devel 3 Dynamic facial reconstruction / aesthetics \\
The masseteric nerve transfer is considered to be useful in \\
patients with bilateral facial palsy, patients with \\
limited facial movement on the healthy side, patients \\
with major asymmetry at rest, or patients who want to \\
avoid a two-stage procedure. \\
Displacement, velocity, and symmetrical movement of \\
the commissure is greater in patients with a masseteric \\
nerve transposition compared to a cross-facial nerve \\
graft. \\
Spontaneous movement of free flap reconstruction using \\
masseteric nerve reanimation is not observed in most \\
patients, although some studies report a high rate of \\
spontaneous movement. \\
Ref $(7,11,12,17,18,23,24,32)$
\end{tabular}

\begin{tabular}{l}
\hline Level $3 \quad$ Dynamic facial reconstruction / aesthetics \\
Complications of nerve grafting with flap transfers \\
include hematomas, abscesses and free flap failure \\
$(1-2 \%)$. \\
Most patients (91\%) needed additional surgical revisions \\
after free flap transfers, such as debulking or \\
reanchoring of the muscle graft. \\
Long-term results of free-muscle transfer for smile \\
restoration in children and adults show good muscle \\
function and aesthetic outcomes that improve over \\
time. \\
$\operatorname{Ref}(7,17,18,23,24,26,27)$ \\
\hline
\end{tabular}

\section{Considerations}

\section{- Overall quality of evidence}

Most conclusions were based on case series or retrospective cohort studies without controls. This type of non-comparative research is graded as level 3. The proposed treatment algorithm by Lindsay et al. (16) and the advice to achieve facial animation with cross-facial nerve grafting were based on expert opinion and are therefore graded on level 4 .

\section{- Balance of benefits and harms}

The preferred method of treatment for the various functional and aesthetic problems related to facial nerve palsy in patients with CFM is based on the balance of the potential benefits and harms and the selection of a certain type of treatment should therefore be made together with the patient. Some procedures, such as free muscle transfer, involve a high burden for the patient, long surgery, donor-side morbidity, and risk of complications. Nevertheless, the final results may be better compared to less burdensome treatment. It is essential to inform patients about the risk and potential benefits for optimal shared decision making.

\section{- Outcome importance}

A considerable number of patients with CFM experience facial palsy. If functional problems occur, treatment is essential to prevent further harm such as blindness. In case of aesthetic problems, treatment may still be of great importance to patients. Treatment should be considered after psychosocial support by a psychologist.

\section{- Costs and resources}

The impact on costs and resources of the given recommendations will vary per member state, depending on the available care providers and facilities. The recommendations concern the essential requirements for adequate treatment of patients with CFM and should thus be implemented. Costs are lowest and resources are most efficiently used when care for craniofacial disorders is centralised in a limited number of expert centres per member state. A general rule that can be applied is one expert centre per 10 million inhabitants.

\section{- Inequity of the recommendation}

The goal of the European Reference Networks (ERNs) is to eliminate inequality within Europe with regard to care for patients with rare diseases. At present, not every member state offers an expert centre for CFM, or the level of provided care does not (yet) meet all the requirements that are outlined in this guideline. By defining the baseline of required care for CFM, this guideline will help these member states to reach the appropriate level. The ERN on craniofacial anomalies and ENT disorders (ERN-CRANIO) can guide a patient in Europe to the available centres of expertise (www.ern-cranio.eu) and can support care providers with diagnosis and treatment advice.

\section{- Feasibility of the recommendation}

Recommendations refer to the general requirements for delivering optimal healthcare and are discussed with members from participating European countries. Quality of care was paramount in the discussions. Centralisation is proposed as one of the core values. However, in some countries the national organisation of healthcare might impede centralisation. National implementation of the ERNs that fits the situation of each country is necessary. For the member states with the lowest number of inhabitants, the establishment of a craniofacial centre might not be feasible, and collaboration with a craniofacial centre in the surrounding countries can be an option.

\section{- Acceptability of the recommendation}

It is expected that all stakeholders want to apply and will be applying the recommendations, because they are employed in ERNacknowledged institutions. National implementation plans are necessary to ensure that recommendations fit the situation in each country. In addition, not all countries participating in the ERNCRANIO are represented in the guideline development group, and new members will join within the coming years. For these countries, acceptance and implementation of ERN guidelines such as this guideline on CFM is obligatory.

\section{Rationale of the recommendations}

Treatment of lagophthalmos is important in patients with facial palsy to prevent severe corneal pathology, endophthalmitis or even blindness. For all different indications, various techniques have been described, all with their own benefits and harms. Figure 1 shows a flowchart of the recommendations on the various treatment modalities that can be performed to treat the facial nerve deformity based on the multiple indications. This makes the choice for a certain type of treatment very patient specific and the choice for treatment should be made together with the patient. In patients with bilateral CFM, the masseter nerve could be used for dynamic 
reanimation. Although good displacement, velocity, and symmetrical movement of the commissure can be achieved, most patients won't be able to smile naturally. Therefore, cross-facial nerve grafting is regarded as the most optimal treatment. Cross-facial nerve grafting with functional muscle transfer can be offered from the age of four to five onwards $(33,34)$. To achieve optimal results a facial physical therapist is considered an integral part of the management of facial palsy. It is important to provide information on the various treatment modalities help patients to make a wellconsidered choice. Another important aspect is the involvement of psychosocial support by a psychologist prior to and after treatment, especially in patients with aesthetic concerns.

\section{Recommendations}

\section{Eye}

- Correct lagophthalmos due to facial palsy in patients with craniofacial microsomia with placement of a gold weight or platinum chain, muscle transfers and/or tendon slings, or cross-facial nerve grafting.

- Tarsorrhaphy as a treatment for lagophthalmos in patient with craniofacial microsomia is discouraged.

\section{Oral}

- Start with the injection of botulinum toxin in the nonaffected depressor labii inferioris muscle if therapy is indicated in patients with craniofacial microsomia and asymmetrical lip depression due to facial palsy.

- Consider myomectomy of the non-affected depressor labii inferioris muscle if the outcome of treatment with botulinum toxin injections are satisfactory.

- Consider the use of dynamic techniques such as digastric muscle transfers if the outcome of treatment with botulinum toxin injections are not satisfactory.

- Perform imaging of the digastric muscle prior to surgical muscle transfer due to the high prevalence of agenesis of the anterior belly of the digastric muscle.

\section{Aesthetics}

- Psychological input is required pre- and post-operatively to monitor expectation and acceptance.

- Strive for spontaneous facial animations by using a cross-facial nerve graft with a free flap.

- Consider functional muscle transfer from the age of four onwards.

- Reserve the use of the masseteric nerve to innervate the free muscle transfer for patients in whom cross-facial nerve grafting is not favourable, in bilateral cases, or as a babysit procedure.

- Consider the use of regional muscle transfers to achieve facial animation in patients with craniofacial microsomia and facial palsy if cross-facial nerve grafting with free muscle transfers is not preferred.

- A facial physical therapist is part of the multidisciplinary team.

- Collect clinician- and patient-reported outcome measures pre- and posttreatment.
Figure 1. Flowchart recommendations treatment facial palsy in craniofacial microsomia

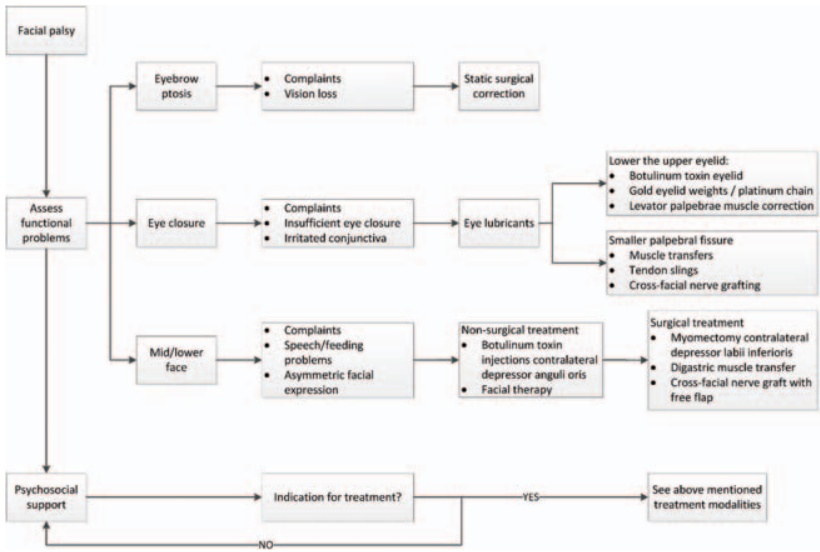

\section{Research gap}

Future studies on the timing of treatment of facial nerve deformity in patients with CFM that addresses psychosocial, aesthetic and functional concerns, and other facial surgeries in these patients, could help establish a protocol on optimal timing of treatment.

\section{References}

1. Caron C, Pluijmers BI, Wolvius EB, Looman CWN, Bulstrode $\mathrm{N}$, Evans RD, et al. Craniofacial and extracraniofacial anomalies in craniofacial microsomia: a multicenter study of 755 patients'. J Craniomaxillofac Surg. 2017;45(8):1302-10.

2. Bassila MK, Goldberg R. The association of facial palsy and/ or sensorineural hearing loss in patients with hemifacial microsomia. Cleft Palate J. 1989;26(4):287-91.

3. Manara R, Brotto D, Ghiselli S, Mardari R, Toldo I, Schifano $\mathrm{G}$, et al. Cranial Nerve Abnormalities in Oculo-AuriculoVertebral Spectrum. AJNR Am J Neuroradiol. 2015;36(7): 1375-80.

4. Carvalho GJ, Song CS, Vargervik K, Lalwani AK. Auditory and facial nerve dysfunction in patients with hemifacial microsomia. Arch Otolaryngol Head Neck Surg. 1999;125(2): 209-12.

5. Birgfeld CB, Heike C. Craniofacial microsomia. Semin Plast Surg. 2012;26(2):91-104.

6. Reddy S, Redett R. Facial paralysis in children. Facial Plast Surg. 2015;31(2):117-22.

7. Roy M, Klar E, Ho ES, Zuker RM, Borschel GH. Segmental Gracilis Muscle Transplantation for Midfacial Animation in Mobius Syndrome: A 29-Year Experience. Plast Reconstr Surg. 2019;143(3):581e-91e.

8. Fattah A, Borschel GH, Manktelow RT, Bezuhly M, Zuker RM. Facial palsy and reconstruction. Plast Reconstr Surg. 2012;129(2):340e-52e.

9. Hoofd-Halsgebied NVvK-heHvh. Richtlijn Idiopathische Perifere Aangezichtsverlamming (IPAV). 2009.

10. Luijmes RE, Pouwels S, Beurskens CH, Kleiss IJ, Siemann I, Ingels KJ. Quality of life before and after different treatment modalities in peripheral facial palsy: A systematic review. Laryngoscope. 2017;127(5):1044-51.

11. Bos R, Reddy SG, Mommaerts MY. Lengthening temporalis myoplasty versus free muscle transfer with the gracilis flap for long-standing facial paralysis: A systematic review of outcomes. J Craniomaxillofac Surg. 2016;44(8):940-51. 
12. Hontanilla B, Olivas J, Cabello A, Marre D. Cross-Face Nerve Grafting versus Masseteric-to-Facial Nerve Transposition for Reanimation of Incomplete Facial Paralysis: A Comparative Study Using the FACIAL CLIMA Evaluating System. Plast Reconstr Surg. 2018;142(2):179e-91e.

13. Loyo M, Jones D, Lee LN, Collar RM, Molendijk J, Boahene $\mathrm{KD}$, et al. Treatment of the periocular complex in paralytic lagophthalmos. Ann Otol Rhinol Laryngol. 2015;124(4): 273-9.

14. Terzis JK, Karypidis D. The outcomes of dynamic procedures for blink restoration in pediatric facial paralysis. Plast Reconstr Surg. 2010;125(2):629-44.

15. Chen CK, Tang YB. Myectomy and botulinum toxin for paralysis of the marginal mandibular branch of the facial nerve: a series of 76 cases. Plast Reconstr Surg. 2007;120(7): 1859-64.

16. Lindsay RW, Edwards C, Smitson C, Cheney ML, Hadlock TA. A systematic algorithm for the management of lower lip asymmetry. Am J Otolaryngol. 2011;32(1):1-7.

17. Chuang DC, Lu JC, Chang TN, Laurence VG. Comparison of Functional Results After Cross-Face Nerve Graft-, Spinal Accessory Nerve-, and Masseter Nerve-Innervated Gracilis for Facial Paralysis Reconstruction: The Chang Gung Experience. Ann Plast Surg. 2018;81(6S Suppl 1):S21-S9.

18. Gousheh J, Arasteh E. Treatment of facial paralysis: dynamic reanimation of spontaneous facial expression-apropos of 655 patients. Plast Reconstr Surg. 2011;128(6):693e-703e.

19. Harrison DH, Grobbelaar AO. Pectoralis minor muscle transfer for unilateral facial palsy reanimation: an experience of 35 years and 637 cases. J Plast Reconstr Aesthet Surg. 2012;65(7):845-50.

20. Hontanilla B, Marre D. Eyelid reanimation with gold weight implant and tendon sling suspension: evaluation of excursion and velocity using the FACIAL CLIMA system. J Plast Reconstr Aesthet Surg. 2013;66(4):518-24.

21. MacQuillan A, Biarda FU, Grobbelaar A. The incidence of anterior belly of digastric agenesis in patients with hemifacial microsomia. Plast Reconstr Surg. 2010;126(4):1285-90.

22. Baheerathan N, Ethunandan M, Ilankovan V. Gold weight implants in the management of paralytic lagophthalmos. Int J Oral Maxillofac Surg. 2009;38(6):632-6.

23. Banks CA, Jowett N, Iacolucci C, Heiser A, Hadlock TA. FiveYear Experience with Fifth-to-Seventh Nerve Transfer for Smile. Plast Reconstr Surg. 2019;143(5):1060e-71e.

24. Bianchi B, Ferri A, Ferrari S, Copelli C, Salvagni L, Sesenna E. The masseteric nerve: a versatile power source in facial animation techniques. Br J Oral Maxillofac Surg. 2014;52(3): 264-9.

25. Panossian A. Lengthening Temporalis Myoplasty for SingleStage Smile Reconstruction in Children with Facial Paralysis. Plast Reconstr Surg. 2016;137(4):1251-61.

26. Terzis JK, Olivares FS. Long-term outcomes of free muscle transfer for smile restoration in children. Plast Reconstr Surg. 2009;123(2):543-55.

27. Terzis JK, Olivares FS. Long-term outcomes of free-muscle transfer for smile restoration in adults. Plast Reconstr Surg. 2009;123(3):877-88.

28. Volk GF, Pantel M, Guntinas-Lichius O. Modern concepts in facial nerve reconstruction. Head Face Med. 2010;6:25.

29. Rahman I, Sadiq SA. Ophthalmic management of facial nerve palsy: a review. Surv Ophthalmol. 2007;52(2):121-44.

30. Majid OW. Clinical use of botulinum toxins in oral and maxillofacial surgery. Int J Oral Maxillofac Surg. 2010;39(3): 197-207.
31. Barr JS, Katz KA, Hazen A. Surgical management of facial nerve paralysis in the pediatric population. J Pediatr Surg. 2011;46(11):2168-76.

32. Terzis JK, Konofaos P. Nerve transfers in facial palsy. Facial Plast Surg. 2008;24(2):177-93.

33. Ghali S, MacQuillan A, Grobbelaar AO. Reanimation of the middle and lower face in facial paralysis: review of the literature and personal approach. J Plast Reconstr Aesthet Surg. 2011;64(4):423-31.

34. Leckenby J, Grobbelaar A. Smile restoration for permanent facial paralysis. Arch Plast Surg. 2013;40(5):633-8.

\section{CHAPTER 5. SURGICAL TREATMENT}

\subsection{Soft tissues \\ Introduction}

One of the relevant factors leading to asymmetry in patients with craniofacial microsomia (CFM) is soft tissue deficiency. This is mainly characterised by a lack of subcutaneous fat or a deficiency of the musculature. A large retrospective study of 755 patients with CFM reported a soft tissue deficiency in $82 \%$ of all patients: mild (S1) in $43 \%$, moderate (S2) in $30 \%$, and severe (S3) in $9 \%$ of patients (1). In the O.M.E.N.S. classification, which is often used to categorise patients with CFM, the soft tissue score (S) is originally graded from $\mathrm{S} 0$ - no obvious deficiency, to $\mathrm{S} 1$ - minimal subcutaneous/muscle deficiency, S2 - moderate, between S1 and S3, and S3 - severe soft tissue deficiency due to subcutaneous and muscular hypoplasia (2). The phenotypic assessment tool by Birgfeld et al. translated this to $\mathrm{S} 0$ - normal, $\mathrm{S} 1$ - minimal soft tissue deficiency, S2 - moderate soft tissue deficiency, and S3 - severe soft tissue deficiency (3).

The aim of treatment is to restore a normal shape of the face and strive for symmetry (4). Various types of treatment are currently used to treat soft tissue deficiency, including fat grafting, pedicles flaps, free tissue transfers, or alloplastic implants. The main factors in the selection of a type of treatment are the severity of the soft tissue deficiency, the presence of other (bony) facial deformities, and the patient's age.

To make recommendations on the indications for treatment and the optimal treatment strategy to address soft tissue deficiency in patients with CFM, the following questions were posed:

5.3.1 What is the indication for surgical treatment of soft tissue deficiency in patients with craniofacial microsomia?

5.3.2 What is the most optimal treatment modality for soft tissue deficiency in patients with craniofacial microsomia related to severity and its timing?

Recommendations on the indications and optimal treatment strategy for soft tissue deficiency in patients with CFM are important to optimise outcomes. It is essential to inform the patient on the potential harms and benefits of treatment and to ensure that the patient has a realistic view on what can be expected, especially if the indication for treatment is patient specific and based on aesthetic concerns. Shared decision making is only possible if the patient is well informed. The aim of these questions is to guide surgeons and to help patients in the choice for treatment.

\section{Literature search}

A systematic search of literature was performed to identify all available literature on CFM and synonyms. The search was conducted in Embase, Pubmed/Medline Ovid. The full search strategy is reported in the supplementary material. 
Inclusion and exclusion criteria:

Type of studies - Original articles

- Systematic review of sufficient quality:

- The question in the systematic review

matches the question of the guideline.

- The search of the systematic review was conducted in at least two relevant

databases, such as the Cochrane Library,

Medline/Pubmed.

- The full search strategy was reported.

- No relevant items were missing in the search strategy.

Type of patients - Patients with craniofacial microsomia

\begin{tabular}{ll}
\hline Subject & - Treatment of soft tissue deficiency \\
\hline Exclusion criteria & - Original studies with $<10$ included patients \\
& - Articles published before 1980 \\
& - Case reports \\
& - Expert opinion \\
& - Letters \\
& - Editorials \\
\hline
\end{tabular}

Literature was screened on title and abstract. Ten studies that addressed (surgical) treatment of soft tissue deficiency in patients with CFM were included. Seven of these articles were included in a systematic review by Sinclair et al. (4). The other two studies included were a narrative review by Birgfeld and Heike (5) and a prospective cohort study on autologous free fat grafting by Denadai et al. (6). The systematic review of Sinclair et al. studied the surgical techniques used to treat soft tissue deficiency in patients with CFM (4). Case reports, case control studies, retrospective cohort studies, and non-randomised prospective studies that addressed treatment of soft tissue deficiency in patients with CFM were included. A total of 38 articles were included. Although 27 of the 38 original articles in this systematic review included fewer than 10 patients, each treatment modality described in the review included $>10$ patients due to combining of data. Therefore, the full systematic review was included in this chapter.

5.3.1 What is the indication for surgical treatment of soft tissue deficiency in patients with craniofacial microsomia?

The literature search for this question was directed towards more specific research questions related to clinical problems, symptoms, treatment options, consequences of not treating, and requirements for treating soft tissue deficiency (Appendix 1, http://links.lww. $\mathrm{com} / \mathrm{SCS} / \mathrm{B} 697)$. Unfortunately, no literature on the indication for surgical treatment of soft tissue deficiency in patients with CFM is available. Therefore no conclusions were written. Considerations were only based on expert opinion.

\section{Considerations}

\section{- Overall quality of evidence}

Because there is no literature available the recommendations are based on expert opinion with a low quality of evidence.

\section{- Balance of benefits and harms}

A considerable number of patients with CFM have a deficiency of the soft tissue. This deficiency, in combinations with other (aesthetic) concerns that may be present in patients with CFM, may lead to psychosocial problems. The aesthetic differences with other, 'healthy' persons can lead to low self-esteem, bullying, or negative feelings. This underlines the importance of psychosocial support for all patients with CFM, which should start during childhood.

\section{- Outcome importance}

No literature is available on the importance of correcting soft tissue deficiency in patients with CFM. Patients with CFM often experience multiple difficulties and the facial deficiency/asymmetry is usually not solely due to soft tissue deficiency. Nevertheless, a deficiency of the facial soft tissue, mild or severe, can have a big impact on the patient. Aesthetic concerns could lead to psychosocial problems in some patients. Treatment of soft tissue deficiency may help patients to feel less 'atypical'.

\section{- Costs and resources}

The impact on costs and resources of the given recommendations will vary per member state, depending on the available care providers and facilities. The recommendations concern the essential requirements for adequate treatment of patients with CFM and should thus be implemented. Costs are lowest and resources are most efficiently used when care for craniofacial disorders is centralised in a limited number of expert centres per member state. A general rule that can be applied is one expert centre per 10 million inhabitants.

\section{- Inequity of the recommendation}

The goal of the European Reference Networks (ERNs) is to eliminate inequality within Europe with regard to care for patients with rare diseases. At present, not every member state offers an expert centre for CFM, or the level of provided care does not (yet) meet all the requirements that are outlined in this guideline. By defining the baseline of required care for CFM, this guideline will help these member states to reach the appropriate level. The ERN on craniofacial anomalies and ENT disorders (ERN-CRANIO) can guide a patient in Europe to the available centres of expertise (www.ern-cranio.eu) and can support care providers with diagnosis and treatment advice.

\section{- Feasibility of the recommendation}

Recommendations refer to the general requirements for delivering optimal healthcare and are discussed with members from participating European countries. Quality of care was paramount in the discussions. Centralisation is proposed as one of the core values. However, in some countries the national organisation of healthcare might impede centralisation. National implementation of the ERNs that fits the situation of each country is necessary. For the member states with the lowest number of inhabitants, the establishment of a craniofacial centre might not be feasible, and collaboration with a craniofacial centre in the surrounding countries can be an option.

\section{- Acceptability of the recommendation}

It is expected that all stakeholders want to apply and will be applying the recommendations, because they are employed in ERNacknowledged institutions. National implementation plans are necessary to ensure that recommendations fit the situation in each country. In addition, not all countries participating in the ERNCRANIO are represented in the guideline development group, and new members will join within the coming years. For these countries, acceptance and implementation of ERN guidelines such as this guideline on CFM is obligatory.

\section{Rationale of the recommendations}

The indication for treatment of soft tissue deficiency in patients with CFM is based on aesthetic problems. The aim of treatment is to strive for facial symmetry. Since the indication for treatment is largely aesthetic and psychosocial with few functional problems, it is essential that the patient is well-informed about the potential risks 
of therapy. Psychosocial help or therapy may help patients to learn to cope with their aesthetic differences. Additionally, psychosocial help enhances the ability to make a well-considered decision to choose whether or not to have treatment. All patients with CFM should be provided with psychosocial support, as is recommended in Chapter 5.3.2. The ICHOM Standard Set for Craniofacial Microsomia advises to assess any difficulties with facial form/asymmetry by using the CleftQ appearance questionnaire at age 8,12 , and 22 .

\section{Recommendations}

- The indication for surgical treatment of soft tissue deficiency in patients with craniofacial microsomia is mainly aesthetic. Inform the patient about the potential benefits and harms to ensure that the patient has a realistic view of what can be expected.

- Patients' difficulties with facial form/asymmetry should be assessed with the CleftQ Appearance at age 8, 12, and 22 .

\section{Research gap}

There is a lack of knowledge on the effectiveness of and patients' reported satisfaction with soft tissue augmentation in patients with CFM. Future research on this topic could help doctors to support patients in the decision-making process for potential soft tissue augmentation. Patient outcome measures, such as FaceQ kids, could be used to evaluate patients perspective on their soft tissue deficiency.

5.3.2 What is the most optimal treatment modality for soft tissue deficiency in patients with craniofacial microsomia related to severity and its timing?

The literature search for this question was directed towards more specific research questions related to treatment options, complications, advantages and disadvantages, short and long term results and treatment burden for treating soft tissue (Appendix 1, http:// links.lww.com/SCS/B697). The surgical treatment modalities were divided into five groups: pedicled flaps, functional reconstruction, alloplastic reconstruction, free flaps, and structural fat grafting. Timing refers to the age of the patient. Included patient outcomes were (psychological) impact of the treatment, complications and satisfaction with the result. Literature on alloplastic reconstructions was scarce and no conclusions on this treatment modality could be drawn.

\section{Review of literature}

In 2019, a systematic review by Sinclair et al. was published that studied the surgical techniques used to treat soft tissue deficiency in patients with CFM (4).

\section{Pedicled flap}

Five articles described a total of thirteen patients with CFM who were treated with a pedicled flap to correct the facial soft tissue deficiency. The mean age of treatment with a pedicled flap was 12.3 years (range 3-27 years). Various pedicled flaps were used: submental flap $(n=3)$, a composite osteomyocutaneous flap based off of the thoracodorsal artery $(n=4)$, a pedicled parietal osteofascial flap $(n=2)$, and a pedicled temporoparietal fascial flap $(n=4)$. The outcomes of treatment were specified in three articles that were included in the review, and were reported to be 'acceptable', 'satisfactory' or a 'good result'. No quantitative outcomes or complication rates were specified and no information about the need for revisional surgery to improve final aesthetics was available.

\section{Alloplastic reconstruction}

The systematic review by Sinclair et al. included one article by Liu et al. that assessed the use of alloplastic materials for soft tissue augmentation, which included thirteen patients with CFM (7). However, no outcomes, complications, severity of the soft tissue deficiency, or location of the implant were reported.

\section{Free flaps}

The use of free flaps was studied in 24 articles including 129 patients with CFM. The severity of the soft tissue deficiency was specified in only one of the 24 studies, which reported a 'severe soft tissue deficiency' in the treated patients. Therefore, the indication for treatment was unknown. The mean age of treatment was 19.0 years (range 2.5-29 years). Most frequently used flaps were the parascapular fasciocutaneous flap $(n=67)$ and the inframammary extended circumflex scapular flap $(n=18)$, although in more recent literature perforator flaps were often preferred. The parascapular fasciocutaneous flap is often used due to its larger size, reliable harvest, and long pedicle (4). Follow-up varied from seven months to seven years and most studies did not report the length of followup. Outcomes were satisfactory or positive in most studies. Most frequently reported complications included hematoma $(n=7)$, partial flap loss $(n=2)$ or donor site infection $(n=1)$. One patient had a total flap loss. Debulking procedures were needed in 28 flaps $(20 \%)$. Two studies mentioned that fat grafting was indicated after free flap reconstruction. The indication for fat grafting in these patients, which may be to correct a low volume of the free flap or sagging of the flap, was not provided in the manuscript.

According to Birgfeld and Heike (5), patients with severe soft tissue deficiencies can best be treated with an adipofascial free flap, such as the scapula, parascapular, groin, omentum, anterolateral thigh, and deep inferior epigastric perforator flap. Drawbacks of the use of free flaps are the need for microsurgical skills, length of the procedure, possible need for debulking, and the donor-site morbidity and scarring (5). Free tissue flaps may sag over time or disrupt normal facial movement.

\section{Fat grafting}

Structural fat grafting to correct the soft tissue deficiency in patients with CFM was addressed in eight articles included in the systematic review. The mean age of treatment was 16.3 years (range 3 - 25 years) and patients needed a mean of 2.7 fat grafting sessions (range 1-6 sessions). Three studies used quantitative analysis to study the outcomes of fat grafting and all three studies reported a (statistically significant) increase in facial symmetry (facial symmetry from $75-86 \%$ to $95-99 \%$, $\mathrm{p}<0.003$ ). Complications of treatment were specified in one study, which reported a $5 \%$ complication rate which included infections and contour irregularities.

Birgfeld and Heike (5) argued that the advantages of fat grafting include minimal scarring and donor-site morbidity, precision of the treated location, and it is less likely to droop or disrupt normal facial movement compared to free tissue grafts. However, multiple sessions may be needed to achieve optimal results. Birgfeld and Heike prefer to use fat grafting to improve facial symmetry during childhood and adolescence and combine fat grafting with other procedures.

To assess fat graft retention in patients with craniofacial contour deformities, a prospective cohort study was undertaken by Denadai et al. (6). Pre- and postoperative soft tissue thickness was measured using ultrasound linear measurements. A total of 142 patients were included; 46 of these patients had CFM. No complications were reported. After the first three months of treatment, a significant and progressive reduction of soft tissue thickness was found 
$(p=>0.05)$. However, these rates stabilised from three to twelve months postoperatively $(\mathrm{p}=>0.05)$. Confounding factors such as postoperative swelling and persistent inflammation may have contributed to the reduction of soft tissue thickness in the first three months. The retention rate of the fat graft was $67.7 \%$. Multivariate analysis showed that patients' age, previous craniofacial bone surgery at the site of fat grafting, and the grafted volume are all independent negative predictors of fat graft retention $(p=<0.05)$. The higher retention rates in paediatric patients compared to adults advocate fat grafting in young patients to achieve facial symmetry, according to the authors (6).

In the systematic review by Sinclair et al., the complication rate of free tissue transfers was compared to fat grafting (4). Patients who had a free tissue transfer had significantly more complications compared to patients who underwent fat grafting $(27.1 \%$ vs $4.2 \%, \mathrm{p}=<0.001$, odds ratio 6.7). However, the authors state that it is difficult to compare the effectiveness of different reconstructive modalities due to differences in reported data, soft tissue deficiency, volume of the flap reconstruction, or quantity of fat grafted. One of the included studies, by Tanna et al. (8), compared free tissue transfer to serial fat grafting and included 31 patients with CFM. Patients with CFM who had fat grafting reported fewer complications ( $5 \%$ vs $12 \%$ ) and had a posttreatment symmetry score of $99 \%( \pm 5.4 \%)$ compared to $121 \%( \pm 7.9 \%)$ in patients with a free tissue transfer. The postreconstruction symmetry score was significantly closer to $100 \%$ in patients with CFM who had fat grafting compared to a free tissue transfer $(p=0.05 \%)$. However, no statistically significant difference was found in patient and physician satisfaction between the two groups. The symmetry analysis and patient and physician satisfaction were measured in the follow-up period, the length of which was not specified. Free tissue transfers achieved a higher (in fact too high) amount of volume augmentations compared to fat grafting $(\mathrm{p}=0.05)$. Tanna et al. concluded that free tissue transfers are considered to be the appropriate management in adolescent patients with significant soft tissue hypoplasia and that fat grafting can be used in growing patients combined with other surgical interventions to improve the craniofacial contour (8). This conclusion was underlined by the authors of the systematic review. Sinclair et al. (4) stated that a minor to moderate soft tissue deficiency can be treated by serial fat grafting, but major soft tissue deficiencies should be treated with a fasciocutaneous free tissue transfer. This may be followed with subsequent fat grafting if indicated. Additionally, surgeons should be aware of the possibility of alterations in vessel anatomy in case of free tissue transfers in patients with CFM.

\section{Conclusions}

Level 3

Pedicled flaps

Pedicled flaps have been used to treat soft tissue deficiency in patients with craniofacial microsomia in a very small number of patients $(n=13)$ and therefore $\operatorname{Ref}(4)$ it is difficult to comment on results.

Level 3

\section{Free flaps}

The results of free tissue transfers to treat soft tissue deficiency in patients with craniofacial microsomia are considered to be satisfactory or positive in most studies.

Complications after free tissue transfer occur in $27 \%$ of patients and include hematoma, partial or total flap loss, infections, or sagging.

Debulking procedures of the free tissue graft are needed in $20 \%$ of the patients with craniofacial microsomia. $\operatorname{Ref}(4,5)$
Level 3
Free tissue transfer versus fat grafting
The severity and rate of complications of free tissue transfers is significantly higher compared to fat grafting in patients with craniofacial microsomia $(27 \%$ vs $4 \%, p=<0.001)$.
Fat grafting leads to a higher level of post-treatment symmetry in patients with craniofacial microsomia compared to free tissue transfers.
$\operatorname{Ref}(4,8)$

\begin{tabular}{|c|c|}
\hline Level 3 & $\begin{array}{l}\text { Fat grafting } \\
\text { Serial fat grafting to correct soft tissue deficiency in } \\
\text { patients with craniofacial microsomia causes a } \\
\text { statistically significant increase in facial symmetry } \\
\text { (facial symmetry from } 75-86 \% \text { to } 95-99 \% \text {, } \\
\text { p }<0.003 \text { ). } \\
\text { The complication rate of fat grafting in patients with } \\
\text { craniofacial microsomia is } 4-5 \% \text { and includes } \\
\text { infections and contour irregularities. } \\
\text { The retention rate of the fat graft is } 68-82 \% \text {, which } \\
\text { stabilises three months after treatment. } \\
\text { Paediatric patients have a higher fat graft retention rate } \\
\text { compared to adults. } \\
\text { Ref }(4-6)\end{array}$ \\
\hline
\end{tabular}

\section{Considerations \\ - Overall quality of evidence}

The conclusions from the literature are mainly based on the systematic review by Sinclair et al. (4), which included 38 articles. The studies included were case reports, case series, or retrospective reviews, all with a level of evidence of 3 to 4 (according to the PRISMA statement (9)). The quality of evidence of conclusions based on this systematic review were therefore graded as level 3 . Denadai et al. (6) performed a prospective cohort study without controls which was graded as level 3 . The study by Tanna et al. (8) was a retrospective cohort study that compared the outcomes of two different groups. The indication for a type of treatment and the length of follow-up were unclear in this study. The quality of evidence was graded as level 3.

\section{- Balance of benefits and harms}

The preferred method of treatment for the aesthetic problems related to soft tissue deficiency in patients with CFM is based on the balance of the potential benefits and harms, and the selection of a certain type of treatment should therefore be made together with the patient. Some procedures, such as free tissue transfers, involve a high burden for the patient, long surgery, donor-side morbidity, and a higher of complications compared to fat grafting. Other drawbacks of this surgery that remain underreported in literature are sagging of the flap in the long run and loss of facial expression. In addition, if complications occur after free tissue transfer these are often severe and involve a high burden for the patient. Free tissue transfers are a suitable solution for patients with a severe soft tissue deficiency, but a substantial volume augmentation can also be achieved with multiple sessions of fat grafting. Limited literature is available on the use of alloplastic materials for soft tissue augmentation in patients with CFM. Complication rates of alloplastic implants such as silicone or polyethylene are similar (4.7\% and $4.8 \%)$ and include infection, hematoma, displacement or prominence $(10,11)$. It is essential to inform patients about the risk and potential benefits for optimal shared decision making. 


\section{- Outcome importance}

It is important to consider timing of treatment and optimal treatment strategy to correct soft tissue deficiency in patients with CFM, because the indication for treatment may vary depending on the patient's age. It is important that patients who prefer treatment to correct the soft tissue deficiency during childhood, adolescence, or adulthood know what types or treatments are available, how these methods might interfere with each other, and what the benefits and harms of these various treatment modalities are. True shared decision making between doctor and patients is only possible if the patient is well informed.

\section{- Costs and resources}

The impact on costs and resources of the given recommendations will vary per member state, depending on the available care providers and facilities. The recommendations concern the essential requirements for adequate treatment of patients with CFM and should thus be implemented. Costs are lowest and resources are most efficiently used when care for craniofacial disorders is centralised in a limited number of expert centres per member state. A general rule that can be applied is one expert centre per 10 million inhabitants.

\section{- Inequity of the recommendation}

The goal of the European Reference Networks (ERNs) is to eliminate inequality within Europe with regard to care for patients with rare diseases. At present, not every member state offers an expert centre for CFM, or the level of provided care does not (yet) meet all the requirements that are outlined in this guideline. By defining the baseline of required care for CFM, this guideline will help these member states to reach the appropriate level. The ERN on craniofacial anomalies and ENT disorders (ERN-CRANIO) can guide a patient in Europe to the available centres of expertise (www.ern-cranio.eu) and can support care providers with diagnosis and treatment advice.

\section{- Feasibility of the recommendation}

Recommendations refer to the general requirements for delivering optimal healthcare and are discussed with members from participating European countries. Quality of care was paramount in the discussions. Centralisation is proposed as one of the core values. However, in some countries the national organisation of healthcare might impede centralisation. National implementation of the ERNs that fits the situation of each country is necessary. For the member states with the lowest number of inhabitants, the establishment of a craniofacial centre might not be feasible, and collaboration with a craniofacial centre in the surrounding countries can be an option.

\section{- Acceptability of the recommendation}

It is expected that all stakeholders want to apply and will be applying the recommendations, because they are employed in ERNacknowledged institutions. National implementation plans are necessary to ensure that recommendations fit the situation in each country. In addition, not all countries participating in the ERNCRANIO are represented in the guideline development group, and new members will join within the coming years. For these countries, acceptance and implementation of ERN guidelines such as this guideline on CFM is obligatory.

\section{Rationale of the recommendations}

The outcomes of fat grafting in literature are considered to be good with relatively low complication rates and a low burden for the patient. This makes fat grafting the first choice of treatment for most patients in which treatment of soft tissue deficiency is indicated. Especially patients with a mild soft tissue deficiency and all children or adolescents are preferred to be treated with fat grafting. Free tissue transfers are considered to be a good treatment modality for patients with severe soft tissue deformities due to the high volume of soft tissue augmentation. Nevertheless, this type of treatment has more impact on the patient: higher complication rates compared to fat grafting (27\%), a longer procedure, and donor-site morbidity and scarring. Patients often need additional debulking procedures due to overcorrection or fat grafting to optimise the results. The risk for drooping or other long-term effects of free tissue grafts is not reported in literature. This suggests the use of free tissue grafts should be limited; they should solely be used in the treatment of patients with CFM and a severe soft tissue deficiency, particularly if the patients have insufficient fat at the donor sites. Although literature on alloplastic implants for soft tissue augmentation in patients with CFM is very limited, alloplastic implants such as silicone or polyethylene, could be a preferred method to treat soft tissue deficiency in patients with CFM, especially for the mandibular angle. The long-term effects of alloplastic implants for soft tissue augmentation are unknown due to the limited follow-up in most studies. Therefore, alloplastic implants for soft tissue augmentation should not be used in growing patients and placement should be postponed until the patient is eighteen years or older. If placement of alloplastic implants is considered, it is advised to postpone fat grafting procedures after placement of alloplastic implants since it changes facial symmetry. The flowchart of the recommendations for treatment of soft tissue deformity in patients with CFM is shown in Figure 1.

\section{Recommendations}

- Psychological input is required pre- and post-operatively to monitor expectations and acceptance.

- Reconstruct soft tissue deficiencies in patients with craniofacial microsomia with fat grafting from childhood.

- Free tissue transfer is only considered in patients with a very severe soft tissue deficiency.

- Alloplastic implants to correct soft tissue deficiency in patients with craniofacial microsomia are ideally performed at skeletal maturity.

- The use of pedicled flaps for correction of soft tissue deficiency in patients with craniofacial microsomia is strongly discouraged.

- Coordinate the timing of surgical treatment of soft tissue deficiency in patients with craniofacial microsomia with the planning of other surgeries, especially for surgeries that affect facial symmetry such as mandibular surgeries or placement of facial implants.

\section{Research gap}

Future studies on the long-term effects of soft tissue augmentation in patients with CFM could help determine an optimal treatment strategy. Especially treatment with alloplastic implants and free tissue transfers should be studied with special attention to the long-term outcomes. Patient outcome measures, such as FaceQ kids, could be used to evaluate of the patients' perspective on their soft tissue deficiency. 3D stereometry is a good tool to study outcomes of treatment. 
Figure 2. Flowchart recommendations of treatment for the soft tissue deformity in patients with craniofacial microsomia

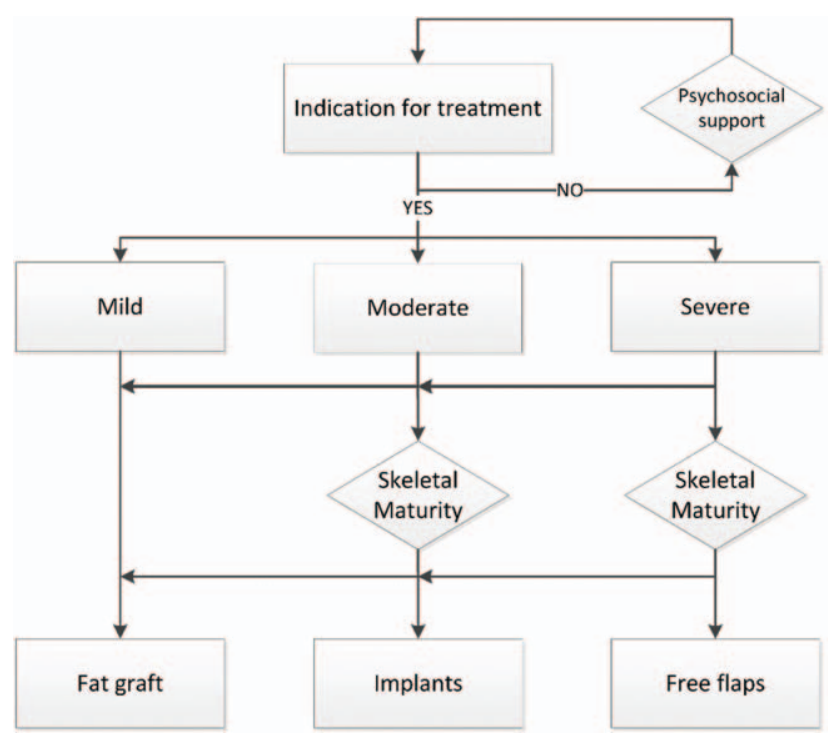

\section{References}

1. Caron C, Pluijmers BI, Wolvius EB, Looman CWN, Bulstrode $\mathrm{N}$, Evans RD, et al. Craniofacial and extracraniofacial anomalies in craniofacial microsomia: a multicenter study of 755 patients'. J Craniomaxillofac Surg. 2017;45(8):130210.

2. Vento AR, LaBrie RA, Mulliken JB. The O.M.E.N.S. classification of hemifacial microsomia. Cleft Palate Craniofac J. 1991;28(1):68-76; discussion 7.

3. Birgfeld CB, Luquetti DV, Gougoutas AJ, Bartlett SP, Low DW, Sie KC, et al. A phenotypic assessment tool for craniofacial microsomia. Plast Reconstr Surg. 2011;127(1): 313-20.

4. Sinclair N, Gharb BB, Papay F, Rampazzo A. Soft Tissue Reconstruction in Patients With Hemifacial Microsomia: A Systematic Review of the Literature. J Craniofac Surg. 2019;30(3):879-87.

5. Birgfeld CB, Heike C. Craniofacial microsomia. Semin Plast Surg. 2012;26(2):91-104.

6. Denadai R, Raposo-Amaral CA, Pinho AS, Lameiro TM, Buzzo CL, Raposo-Amaral CE. Predictors of Autologous Free Fat Graft Retention in the Management of Craniofacial Contour Deformities. Plast Reconstr Surg. 2017;140(1):50e$61 \mathrm{e}$.

7. Liu X, Zhang Y, Wang S, Lei Z, Li X, Fan D. The use of expanded polytetrafluoroethylene in depressed deformities of the face. Exp Ther Med. 2016;12(5):3151-4.

8. Tanna N, Wan DC, Kawamoto HK, Bradley JP. Craniofacial microsomia soft-tissue reconstruction comparison: inframammary extended circumflex scapular flap versus serial fat grafting. Plast Reconstr Surg. 2011;127(2):802-11.

9. Moher D, Liberati A, Tetzlaff J, Altman DG, Group P. Preferred reporting items for systematic reviews and metaanalyses: the PRISMA statement. PLoS Med. 2009;6(7): e1000097.
10. Rojas YA, Sinnott C, Colasante C, Samas J, Reish RG. Facial Implants: Controversies and Criticism. A Comprehensive Review of the Current Literature. Plast Reconstr Surg. 2018;142(4):991-9.

11. Oliver JD, Eells AC, Saba ES, Boczar D, Restrepo DJ, Huayllani MT, et al. Alloplastic Facial Implants: A Systematic Review and Meta-Analysis on Outcomes and Uses in Aesthetic and Reconstructive Plastic Surgery. Aesthetic Plast Surg. 2019;43(3):625-36.

\section{CHAPTER 5. SURGICAL TREATMENT}

\subsection{Microtia \\ Introduction}

Microtia is one of the most common characteristics of patients with CFM as it is seen in $83 \%$ to $88 \%$ of the patients $(1,2)$. Besides external ear malformations, patients may have other malformations such as middle ear malformations or atresia, or the presence of branchial remnants (3). These malformations may lead to hearing problems which are further described in Chapter 4.4 - Hearing difficulties.

In the management of microtia and atresia improving hearing is the most important functional goal, followed by external ear reconstruction if the patient and family feel that is required. The first option for microtia is 'no reconstruction'; however, if the patient and family are keen to proceed, then there are a number of options for treatment, including an external silicone prosthesis attached with either adhesives or an osseointegrated implant, a porous polyethylene buried prosthesis, and ear reconstruction with autologous rib graft. The porous polyethylene prosthesis is implanted by the surgeon and covered by a flap and skin graft (4). Ear reconstruction with rib can be performed using different techniques established by Brent, Firmin and Nagata (4). The Nagata and Firmin technique is often used and involves a two-stage approach. Rib cartilage is harvested and constructed into a detailed ear framework, which is placed in a pocket at the site of the ear. After six months, a second operation is performed to elevate the ear from the head and to create a sulcus.

To offer recommendations on the indications for treatment and the optimal treatment strategy to restore ear deformity in patients with CFM, the following questions were posed:

5.4.1 What is the indication for surgical treatment of ear deformity in patients with craniofacial microsomia?

5.4.2 What is the most optimal treatment modality for ear deformity in patients with craniofacial microsomia related to its timing?

It is essential to inform the patient on the potential harms and benefits of treatment and to ensure that the patient has a realistic view on what can be expected, especially if the indication for treatment is patient specific and based on aesthetic concerns. Shared decision making is only possible if the patient and family are well informed. The aim of these questions is to guide surgeons and to help patients in the choice for treatment.

\section{Literature search}

A systematic search of literature was performed to identify all available literature on CFM and synonyms. The search was conducted in Embase, Pubmed/Medline Ovid. The full search strategy is reported in the supplementary material. 


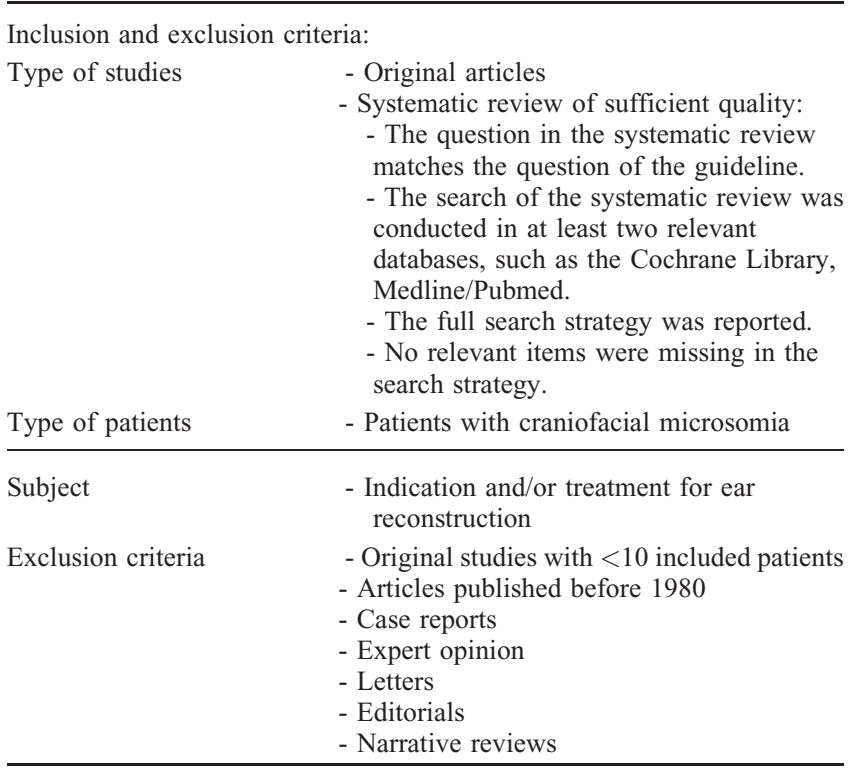

Only literature that described indications, outcomes or complications of ear reconstruction was included. The aim of this chapter is to provide patients and clinicians with guidance on the available treatment options for ear reconstruction in patients with CFM. Therefore, articles that studied surgical techniques in detail were excluded, as this is beyond the scope of this chapter.

Literature was screened on title and abstract. A total of three retrospective cohort studies were included that addressed outcomes on ear reconstruction in patients with CFM: by Mandelbaum et al. (5), Park et al. (6), and Qian et al. (7)

The 'UK Care Standards for the Management of Patients with Microtia and Atresia' (2018) was also included in this chapter (4). This UK guideline on microtia/atresia makes clinically relevant recommendations to improve care, but is not been based on the same methodological criteria as this guideline on CFM. Hence the UK guideline on microtia/atresia did not provide information on the literature search, the inclusion/exclusion criteria, and quality of evidence of the individual studies and conclusions. Nevertheless, the guideline is considered to be clinically relevant, especially since the general quality of evidence of literature on auricular reconstruction is low (8).

An additional literature search was performed to include literature on auricular reconstruction in patients with microtia or atresia. All articles that described indications, outcomes or complications on treatment modalities for auricular reconstruction were included. The full search strategy is shown in the addendum. A total of 369 articles were identified and ten articles met the inclusion criteria and were included in this chapter. One systematic review by Long et al. was included (9), and two cross-sectional studies by Younis et al. (10) and Braun et al. (11), five retrospective cohort studies by Constantine et al. (12), Fu et al. (13), Roos et al. (14), Al Kadah et al. (15), and Si et al. (16), and two case series by Vijverberg et al. (17) and Wright et al. (18)

\subsubsection{What is the indication for surgical treatment of ear} deformity in patients with craniofacial microsomia?

Auricular deformities in patients with CFM are often accompanied by other congenital facial malformations. These deformities can cause aesthetic or psychosocial concerns and may be an indication for surgical reconstruction. As patients with CFM often have multiple facial malformations, the indication for treatment may be more complex compared to patients with isolated microtia/ anotia. Therefore, only literature on patients with CFM was included in this chapter.

\section{Review of literature}

The characteristics of patients with CFM and external ear and hearing problems were studied by Mandelbaum et al. (5) Their retrospective analysis included 68 patients with CFM. Most of these patients had auricular abnormalities (91\%), which were most frequently characterised as grade III microtia (in $75 \%$ of the patients). Data on complications, surgical revisions, aesthetics, and psychosocial function were collected. Psychosocial problems or deficits in school functioning were seen in $30.7 \%$ of the patients. However, no control group was included, which makes this percentage hard to interpret. Data on psychosocial problems were based on retrospective review of medical records and were graded as 'good' or 'poor'. Regression analysis showed that any treatment of hearing loss (which led to significantly better hearing results after treatment) was a significant predictor of good psychosocial outcome (OR, 4.889; 95\% CI, 1.459-16.381; $p=0.010)$. No statistically significant influences of auricular reconstruction on psychosocial functioning were found.

\section{Conclusion}

\begin{tabular}{l}
\hline Level 3 One study did not find a difference in psychosocial \\
functioning after ear reconstruction in patients with \\
craniofacial microsomia $(\mathrm{n}=68)$. \\
$\operatorname{Ref}(5)$
\end{tabular}

\section{Considerations \\ - Overall quality of evidence}

The quality of evidence on the indication for treatment of auricular deformity in patients with CFM was graded on level 3, since the study of Mandelbaum et al. was a retrospective cohort study (5). It is important to keep in mind the retrospective nature of the study and the imperfect method of determining psychosocial problems (by chart review) in assessing the quality of the conclusion. Due to this methodological weakness, the conclusion is considered less strong.

\section{- Balance of benefits and harms}

Microtia can have negative psychosocial consequences due to aesthetic difficulties and the visibility of the disorder. Patients often cover their disfigurement by wearing their hair down, avoiding wet hair or windy weather, avoiding physical activities, social situations, mirrors, photographs and so on (4). A higher prevalence of social problems, aggressive behaviour, and mood disorders are seen in patients with microtia compared to healthy controls (19). The aesthetic differences with other, 'healthy' persons could lead to low self-esteem, bullying, or negative feelings. This underlines the importance of psychosocial support for all patients with CFM, which should start during childhood. Ear reconstruction may be beneficial for patients to feel less 'atypical' and prevent the previously mentioned potential harms.

\section{- Outcome importance}

No literature is available on the importance of correction of the auricular deformity in patients with CFM. Patients with CFM often experience multiple facial differences compared to healthy controls. Nevertheless, it has been shown that the management of microtia or 
anotia has significant positive impact for the patient (20). Aesthetic concerns could lead to psychosocial problems in some patients. Auricular reconstruction may help patients feel less 'atypical'. To assess the benefits of ear reconstruction, the use of a new PROM Ear-Q was agreed at the International Society for Auricular Reconstruction ISAR congress in Beijing in 2017. All surgeons should use the Ear-Q pre- and post operatively.

\section{- Costs and resources}

The impact on costs and resources of the given recommendations will vary per member state, depending on the available care providers and facilities. Treatment with porous polyethylene prosthesis (Medpore) especially could contribute to an increase in costs. Health insurance reimbursement and the willingness to cover these costs may differ between countries. The recommendations concern the essential requirements for adequate treatment of patients with CFM and should thus be implemented. Costs are lowest and resources are most efficiently used when care for craniofacial disorders is centralised in a limited number of expert centres per member state. A general rule that can be applied is one expert centre per 10 million inhabitants.

\section{- Inequity of the recommendation}

The goal of the European Reference Networks (ERNs) is to eliminate inequality within Europe with regard to care for patients with rare diseases. At present, not every member state offers an expert centre for CFM, or the level of provided care does not (yet) meet all the requirements that are outlined in this guideline. By defining the baseline of required care for CFM, this guideline will help these member states to reach the appropriate level. The ERN on craniofacial anomalies and ENT disorders (ERN-CRANIO) can guide a patient in Europe to the available centres of expertise (www.ern-cranio.eu) and can support care providers with diagnosis and treatment advice.

\section{- Feasibility of the recommendation}

Recommendations refer to the general requirements for delivering optimal healthcare and are discussed with members from participating European countries. Quality of care was paramount in the discussions. Centralisation is proposed as one of the core values. However, in some countries the national organisation of healthcare might impede centralisation. National implementation of the ERNs that fits the situation of each country is necessary. For the member states with the lowest number of inhabitants, the establishment of a craniofacial centre might not be feasible, and collaboration with a craniofacial centre in the surrounding countries can be an option.

\section{- Acceptability of the recommendation}

It is expected that all stakeholders want to apply and will be applying the recommendations, because they are employed in ERNacknowledged institutions. National implementation plans are necessary to ensure that recommendations fit the situation in each country. In addition, not all countries participating in the ERNCRANIO are represented in the guideline development group, and new members will join within the coming years. For these countries, acceptance and implementation of ERN guidelines such as this guideline on CFM is obligatory.

\section{Rationale of the recommendations}

The indication for treatment of microtia is primarily aesthetic but has been shown to have large psychosocial benefits in patients with isolated microtia (21). The aim of treatment is to improve the patient's quality of life. Since the indication for treatment is aesthetic, it is essential that the patient is well-informed about the potential risks of therapy. Hearing problems should be assessed and treated, as is explained in Chapter 4.4 - Hearing difficulties. Psychosocial help or therapy may help patients learn to cope with their different appearance. In an even earlier phase, parents of patients should receive information on the possible treatment options for the ear deformity. The multidisciplinary craniofacial team should assess the psychosocial problems and reconstructive options in an individualised manner (4). If treatment is considered, it is essential to inform patients and parents on the expected outcomes and the burden of treatment such as the frequency and length of hospital stays, and risk of complications.

Recommendations

- The indication for auricular reconstruction in patients with craniofacial microsomia is aesthetic and psychosocial. Inform the patient about the potential benefits and harms to ensure that the patient has a realistic view of what can be expected.

- Provide all patients with craniofacial microsomia with psychosocial support.

- Use the PROM Ear-Q pre- and postoperatively to assess benefit of treatment.

\section{Research gap}

There is a lack of knowledge on the effectiveness of and patients reported satisfaction with auricular reconstruction in patients with CFM. Future research on this topic could help doctors support patients in the decision-making process. It is hoped that the use of the new PROM Ear-Q will reduce this gap.

5.4.2 What is the most optimal treatment modality for ear deformity in patients with craniofacial microsomia related to its timing?

The literature search for this question was directed towards more specific research questions related to treatment options, complications, advantages and disadvantages, short and long term results and treatment burden for treatment of microtia/anotia in patients with CFM (Appendix 1, http://links.lww.com/SCS/B697). Besides literature on CFM, the 'UK Care Standards for the Management of Patients with Microtia and Atresia' (2018) and literature identified from the additional search on auricular reconstruction was included (4). Treatment modalities were divided into four groups: external silicone prosthesis attached with adhesives, external silicone prosthesis attached on implants, porous polyethylene implants (Medpore), and auricular reconstruction with autologous rib grafts. Included patient outcomes were (psychological) impact of the treatment, complications and satisfaction with the result.

\section{Review of literature \\ Craniofacial microsomia}

Mendelbaum et al. retrospectively studied 68 patients with CFM, all age $>13$ (5). Microtia was present in $91 \%$ of the patients, and was characterised as type III microtia in $75 \%$ of the patients $(\mathrm{n}=58)$. Total auricular reconstruction with rib grafts was performed in 56 ears, starting at a mean age of 8.5 years (range 5.5-18.7 years). Aesthetic outcomes were assessed by three independent observers on a scale of 1 to 3 . The mean aesthetic outcome score was 5.56 (range 3-9). Patients with severe microtia required more surgeries to achieve optimal results and had lower aesthetic outcome ratings after reconstruction compared to less severe cases (respectively IRR, 4.350; 95\% CI, 2.140-8.843; p < 0.001, and 
IRR, 0.763; 95\% CI, 0.658-0.886; p < 0.001). Complications occurred in $18 \%$ of the patients and were all secondary to wound complications. Most patients $(\mathrm{n}=46)$ needed revisions after completion of ear reconstruction such as deepening of the helical root, tragus, or concha, revision of the lobule, or projection of the new ear. No correlation between the complication rate and the severity of microtia was observed $(\mathrm{p}=0.193)$.

Microtia in patients with CFM is difficult to treat due to additional abnormalities such as facial asymmetry, a low hairline, depression of the temporomandibular joint or temporal bone, and low ear vestige. These characteristics can lead to a deficiency in the skin volume, making covering of the auricular reconstruct difficult (6). Three types of coverage techniques in microtia reconstruction in patients with CFM were studied by Park et al., who included 52 patients with CFM with a mean follow-up of 40 months (range 6 months to 10 years) (6). All patients had auricular reconstruction with rib and were treated with three types of coverage techniques. Results on the comparison of these coverage techniques were considered to be too surgically detailed for this chapter and were not included. However, results on aesthetic outcomes and complications were considered relevant and extracted from the article. The aesthetic outcome of treatment was rated on a 1-10 scale, based on senior author assessment. The mean aesthetic score varied from 6.30 to 8.26. Poor postoperative outcomes were due to large size of the reconstructed ears $(n=11)$, partial resorption of the cartilage framework $(n=11)$, mismatched skin colour $(n=8)$, different ear axis $(n=7)$ or shape $(n=5)$, ear convolution $(n=2)$, or bad-looking scars $(n=2)$. The severity of microtia had no effect on the aesthetic outcome (6).

Qian et al. studied 111 patients with CFM to study the effect of the expanded two-flap method (7). Results on this specific surgical technique were considered to be too detailed for this chapter and were not included. General outcomes of ear reconstruction with rib graft were included. The mean age of the patients was 9 years (range 5 to 27 years) and mean follow-up was 8.3 months (range 5 to 20 months). Aesthetic outcomes of treatment were rated on a $0-15$ scale, and were satisfactory in $92.8 \%$ of the patients, partially satisfactory in $6.3 \%$ of the patients, and unsatisfactory in one patient $(0.9 \%)$. Nine patients $(8.1 \%)$ had surgical complications: tissue expander-related complications and hematoma (1.8\%). Three patients had complications in the second treatment stage: cartilage framework exposure in two patients $(1.8 \%)$ and poor skin graft survival in one patient $(0.9 \%)$.

\section{External silicone prosthesis attached with adhesives}

The UK Guideline Microtia and Anotia considers external silicone ear prosthesis as a good treatment modality for microtia, which can achieve an excellent shape and colour (4). The prosthesis may be attached with adhesives or with implants. No literature on the outcomes or complications of treatment with external silicone prosthesis with adhesives was identified. The benefits and disadvantages of the use of this type of external prosthesis are discussed in the considerations.

\section{External silicone prosthesis attached on osseointegrated implants}

The additional search on non-CFM patients with microtia led to the inclusion of five articles on external prosthesis. Vijverberg et al. studied the outcomes and patient satisfaction of osseointegrated auricular (VXI) implants (17). A total of 31 implants were placed in 11 patients, with a mean age of 44 years (range $13-85$ years) and a minimal follow-up of 7 months (mean follow-up 2.6 years). Soft tissue reaction was observed in $32.2 \%$ of the implants, which all resolved after topical treatment with antibiotic/corticosteroid ointment. All patients reported an improvement in quality of life after receiving the implants with prosthesis, although two patients $(18 \%)$ reported a slight physical disadvantage using the prosthesis (17). Si et al. also reported the troublesome complication of skin reaction (16). All their studied patients $(n=24,25$ ears) who received osseointegrated auricular implants for an external prosthesis had skin reaction. The degree of skin redness, moist, or granulation tissue gradually decreased over time. Almost all patients $(95.8 \%)$ wore the prosthesis for more than eight hours per day (16).

Wright et al. studied the survival rate of auricular implants in 16 patients and 39 implants. The average age at placement was 40 years (range 6 to 76 years) and the mean follow-up was 45 months (range 6 months to 17 years). The survival rate was $100 \%$ as no implant failures occurred. Most patients $(n=13)$ had soft tissue inflammation, which resolved in $54 \%$ of the patients with hygiene support or soft tissue reduction (18).

Younis et al. examined the patient satisfaction in a crosssectional study of 20 patients who completed prosthetic ear reconstruction (average age 37 years, range $17-56$ years) (10). The indication for ear prosthesis was due to a congenital deformity $(\mathrm{n}=10)$, trauma $(\mathrm{n}=9)$ or malignancy $(\mathrm{n}=1)$. Most patients $(\mathrm{n}=14)$ had previous failed autologous reconstruction. Skin problems, such as granulation tissue $(n=14)$ or skin infection $(n=10)$, were often reported. Nevertheless, 14 patients wore the prosthesis daily. The aesthetic appearance of the prosthesis was considered excellent or very good by 17 of the 20 patients. Only 12 of the 20 patients were satisfied with their prosthesis. The authors consider the high rate of skin complications to be the reason for the low overall satisfaction rate (10).

\section{Osseointegrated implant-supported external silicone prosthesis versus porous polyethylene implants (Medpore)}

$\mathrm{Al}$ Kadah et al. reported their experience with silicone or porous polyethylene ear prosthesis in 39 patients (43 implants) with a minimal follow-up of two years (15). Eighteen patients had porous polyethylene implants placed (mean age 19 years, range 8-56 years) and 21 patients had silicone ear protheses fixed on implants (mean age 41 years, range 6-84 years). None of the 18 patients with a porous polyethylene implant saw the implant rejected, although four patients needed revisions due to keloid $(n=1)$, retroauricular adhesion $(\mathrm{n}=2)$, and local infection leading to partial resection of the implant $(\mathrm{n}=1)$. In the silicone prosthesis group, 18 patients $(85 \%)$ wore the prosthesis for at least 10 hours a day. No extrusion of the implants for the external prosthesis was observed. Revision surgeries were needed in five patients due to: exposure of the implants $(n=2)$, infection $(n=1)$, loose screw $(n=1)$, tumour recurrence $(n=1)$. Local skin reaction at the abutment was seen in $71.4 \%$ of the patients $(n=15)$ during follow-up. No skin reactions in the porous polyethylene implant group were reported.

\section{Porous polyethylene implants (Medpore)}

Porous polyethylene implants could yield good aesthetic results with lower burden for the patient compared with autologous reconstruction, although complications such as extrusion or fracture of the implant could be seen in up to $13-15 \%$ of the patients according to the UK microtia/anotia guideline (4). Unfortunately, no literature was provided to validate these numbers and the methodological quality of the articles.

In the study by Al Kadah et al. (described above), no extrusion of the alloplastic implants was observed, although one of the eighteen patients needed partial resection of the implant after local infection (15). 
Braun et al. studied the patient-reported outcome after ear reconstruction with porous polyethylene implants (Medpore) in a crosssectional study (11). A total of 65 patients were included, who all received a questionnaire. Most patients $(84.6 \%)$ were treated due to congenital microtia and 15 patients were found to have CFM. The median age at time of treatment was 18 years (range 4-68 years), and the mean follow-up was 2.9 years (range $0.5-6.0$ years). Adult patients $(n=45)$ reported an overall increase in quality of life after auricular reconstruction $(\mathrm{p}<0.0001)$. Most of these patients $(72.7 \%)$ were satisfied with the aesthetic outcome. Of the $27.3 \%(n=12)$ of the patients who were not satisfied, eight would again decide to have the operation. A negative score, indicating an adverse effect, was reported by $17.8 \%$ of the adults. Most frequently reported complaints by patients include difficulties with scars $(\mathrm{n}=18,40 \%)$, feeling $(\mathrm{n}=17,37 \%)$, shape of the ear $(\mathrm{n}=16,36 \%)$, numbness $(\mathrm{n}=11$, $24 \%)$, skin colour $(n=8,18 \%)$ or position of the ear $(n=4,9 \%)$. No failures leading to removal of the prosthesis were reported. Two thirds of the patients were not limited in their physical activities due to the reconstructed ear and $62.8 \%$ of the patients could sleep on the reconstructed ear (11). Similar results were seen in the paediatric cohort $(n=20)$. An increase in the health-related quality of life was observed $(\mathrm{p}=0.01)$. Most patients $(85 \%)$ and parents of patients $(73.7 \%)$ were satisfied with the aesthetic results, and all patients and parents who were not satisfied would again decide to have the operation. The main complaints were similar to the adults, including scars and feeling. Complaints regarding the position, shape and colour were less frequently reported compared to the adult population (no numbers were reported). One patient psychologically rejected the new ear. Most children had no physical limitations (75\%) and could sleep on the reconstructed ear (85\%) (11).

\section{Porous polyethylene implants (Medpore) versus ear reconstruction with autologous rib graft}

The outcomes of porous polyethylene implants and autologous ear reconstruction with rib were compared by Constantine et al (12). Their retrospective analysis included 35 patients (36 ears) who had complete microtia repair: 17 polyethylene implants (mean age 6.9 years, SD 3.0), 17 reconstructions with rib (mean age 8.0 years, SD 3.8 ), and 2 with both. The follow-up ranged from 2 to 11 years. Two blinded observers rated the aesthetic outcomes of treatment. The mean number of operations was higher in the rib reconstruction group (4.88, SD 1.54) compared to the polyethylene implant group (3.35, $\mathrm{SD} 1.27, \mathrm{p}=0.004)$. One complication occurred in the rib reconstruction group: cartilage exposure. Two complications occurred in the implant group: one infection and one extrusion of the implant. And in the two patients who had both types of reconstruction, two infections and two extrusions of the polyethylene implants occurred. A trend toward more infections and extrusions in the polyethylene group compared to the rib reconstruction group may be present. Concerning the aesthetic outcomes, the rib reconstruction group had significantly better outcomes regarding the definition, shape, and colour match compared to polyethylene implants $(\mathrm{p}=0.05, \mathrm{p}=0.08$, $\mathrm{p}=0.05$ respectively). The size of the ear was better in the implant group $(\mathrm{p}=0.05)$. No statistically significant differences in ear protrusion and location were observed.

\section{Ear reconstruction with autologous rib graft}

Ear reconstruction with autologous rib cartilage has been considered the golden standard in microtia reconstruction for years (4). In the first operation, performed at age nine to ten, the rib cartilage is harvested and constructed into a framework, which is then placed in a pocket at the site of the ear. Six months later, the second procedure is performed in which the ear is elevated from the head. This reconstruction is considered to be durable, showing no softening or shrinkage of the cartilage and having minimal long-term complications (4). The newly reconstructed ears with rib cartilage were found to grow as much as normal ears after reconstruction (after a median follow-up of 5.6 years, range 2.1-10.6 years) (14).

A systematic review on the complications of autologous ear reconstruction with rib was conducted by Long et al. in 2013 (9). A total of 60 articles, including 9,415 patients, were included in the analysis. The overall complication incidence was $16.2 \%$ (range $0-72.9 \%$ ). Complications included: dissatisfying final results $(8.5 \%)$, hypertrophic scar $(6.3 \%)$, asymmetry $(2.1 \%)$, wire or suture extrusion (1.6\%), flap venous congestion (1.6\%), cartilage absorption (1.3\%), facial nerve injury $(1.1 \%)$, frame exposure $(1.0 \%)$, infection $(0.9 \%)$, hematoma $(0.3 \%)$, and grafted skin necrosis $(0.4 \%)$. Leakage or exposure of the tissue expander has an incidence of respectively $1.4 \%$ and $3.5 \%$. Complications at the donor site included atelectasis, pleural tear, chest wall deformity, thoracic scoliosis, and hypertrophic scar. The overall incidence of these complications varied from $5.6 \%$ to $36.1 \%$.

A recent retrospective cohort study of 470 ear reconstructions with rib grafts by Fu et al. (13) reported a complication rate of $9.43 \%$. All patients included $(n=429)$ were treated in the same centre, by the same surgeon. The mean follow-up was 3.7 years ( \pm 2.5 years). Age, sex, and laterality were not associated with the complications $(p>0.05)$. The risk of complications was significantly higher in patients treated with the Nagata technique (12.2\%, 38/311) compared with the Brent technique $(2.98 \%, 4 / 134)$ (OR 6.14, 95\% CI $1.63-$ $23.19 ; \mathrm{p}<0.01)$. The authors suggest that this is presumably the result of a more complicated and stereoscopic technique in the Nagata technique, with a higher risk for vascular compromise of the skin flap compared to the Brent technique (13). Despite the higher risk of complications when using the Nagata technique, the authors advise the use of this technique since it requires fewer surgical stages (two stages) compared to the Brent technique (three to four stages).

Conclusions

Level 3 External silicone prosthesis
Non-craniofacial microsomia patients who wear an
osseointegrated implant- supported external silicone
prosthesis are often satisfied with the result (placed at a
mean age of $37-44$ years).
Implants for an external prosthesis often lead to local skin
reactions (in $32-100 \%$ of patients), which may decrease
the satisfaction with the prosthesis. Treatment with
antibiotic/corticosteroid ointment or increased hygiene is
often successful.
No conclusion on revision- or failure rates of implants can be
drawn, as studies were based on small patient samples
with limited follow-up.
Implants for external silicone prosthesis were placed at a
mean age of 19-44 years (range $8-85$ years) in non-
craniofacial microsomia patients.
Ref $(4,10,15-18)$

Level 3 Porous polyethylene implants (Medpore) (inserted at mean age of $6-18)$

Most patients $(73 \%)$ report an increase in quality of life and are satisfied with the aesthetic result (73-75\%) after ear reconstruction with porous polyethylene implants.

Dissatisfying results were mainly due to complaints regarding scars, hair loss, and numbness.

Extrusion or fracture of the implant was seen in up to 13 $15 \%$ of patients.

Polyethylene implants have better aesthetic outcomes regarding the size of the ear compared to reconstruction with rib, whereas rib-reconstructed ears show better outcomes regarding definition, shape, and colour match. $\operatorname{Ref}(4,11,12,15)$ 


\begin{tabular}{c}
\hline Level $3 \quad \frac{\text { Ear reconstruction with rib in patients with craniofacial }}{\text { microsomia }}$ \\
Patients with craniofacial microsomia and severe \\
microtia required more operations to achieve optimal \\
results compared to patients with less severe microtia. \\
Evidence on the effect of the severity of microtia on the \\
aesthetic outcome of ear reconstruction in patients \\
with craniofacial microsomia is contradictory and no \\
conclusions can be drawn. \\
Reasons for poor postoperative outcomes after ear \\
reconstruction in patients with craniofacial \\
microsomia include: mismatch in size, skin colour, ear \\
axis, shape, or partial resorption of the cartilage \\
framework. \\
Ref $(5,6)$
\end{tabular}

Level 3 Covering techniques for ear reconstruction with rib in
patients with craniofacial microsomia
Various covering techniques can be used for ear
reconstruction in patients with craniofacial
microsomia:
- The embedded technique is associated with good
aesthetic outcomes.
- The fascia flap technique is associated with good
aesthetic outcomes and is often used in patients with
severe craniofacial microsomia. A mismatch in skin
colour is more frequently observed in patients treated
with this technique compared to others.
- The expansion technique is associated with good
aesthetic outcomes, but has worse outcomes compared
to the other techniques.
Ref $(6,7)$

Level 3

Ear reconstruction with rib

Ear reconstruction with rib is considered a durable treatment option with minimal long-term complications. The reconstructed ears show a similar growth pattern to the normal ear.

Ear reconstruction with rib is usually performed at age eight to ten.

The overall complications incidence of ear reconstruction with rib is $16 \%$.

Reported complications include dissatisfying final results (9\%), hypertrophic scar $(6 \%)$, asymmetry $(2 \%)$, wire or suture extrusion (2\%), flap venous congestion ( $2 \%)$, cartilage absorption (1\%), facial nerve injury (1\%).

Donor site complications include pain, atelectasis, pleural tear, chest wall deformity, thoracic scoliosis, and hypertrophic scar.

$\operatorname{Ref}(4,9,13,14)$

\section{Considerations}

\section{- Overall quality of evidence}

The levels of evidence were considered to be level 3 due to the non-comparative nature of most studies. The systematic review by Long et al. on complications of ear reconstruction with rib excluded case reports and case series with fewer than 15 patients. This study is descriptive and non-comparative, leading to a level 3 quality of evidence. Al Kadah et al. and Constantine et al. made a retrospective comparison between two groups: polyethylene implants versus reconstruction with rib (12) and polyethylene implants versus silicone prosthesis (15). Park et al. retrospectively compared three different covering methods for the reconstructed ear (6). Although these studies are comparative, the level of evidence is graded as level 3 since the studies were not randomised and no other comparative studies could be included that supported the conclusions.

\section{- Balance of benefits and harms}

External silicone prosthesis on adhesives

The prosthesis can be attached with adhesives at a young age, although this is controversial. Early use of the prosthesis to let the child get used to it, could make the child feel less-conscious of the prosthesis when school starts. However, no evidence is available that supports this theory. Additionally it may prevent teasing, although no literature is available to support this theory either. Early treatment is likely to be a reaction to parental anxiety rather than clinical need. The use of an external prosthesis in young children could also imply that the deformed ear needs to be camouflaged and could lead to the association of negative feelings (4).

External silicone prosthesis on implants

Advantages of an external silicone prosthesis are the realistic looks and its relatively simple use/placement. Disadvantages include the need for replacement of the implants, cleaning of the pin sites, risk of losing the prosthesis during physical activities, skin colour changes, or problems with the implants (e.g. infection or over-granulation) (4).

If implants are placed for prosthesis attachment, the microtic remnant often needs to be removed. It is important that the patient and family are aware that other forms of ear reconstruction are impaired after this treatment (4).

Alloplastic implants

Reconstruction with Medpore implants is quicker and has a lower burden for the patient compared to autologous ear reconstruction, as no rib cartilage has to be harvested using this technique. In addition, reconstruction with Medpore is less technically challenging compared to autologous reconstruction, although technical expertise is still required to achieve good results (4).

Medpore implants can be placed at a young age (three to five years), although the size of the implants has to be bigger to account for future growth of the contralateral ear (4). At this age, the child is not aware of its facial differences, which makes the indication for this invasive treatment debatable. Also, if an implant is placed, future autologous reconstruction with rib is impaired given the scarring of the local soft tissues. Another important aspect is the risk of complications, especially if the implant is placed in children and if the child engages in contact sports. The complications, such as extrusions or fractures of the implants, are serious and make future reconstruction with other methods challenging. Therefore, it is advised to be cautious in treating children with Medpore implants. If reconstruction with Medpore is indicated, it is advised to postpone treatment until after the age of six since at this age the width of the normal ear is approaching its mature size (22).

Ear reconstruction with rib

The aesthetic results of ear reconstruction with autologous rib grafts are considered to be good in the hands of experienced surgeons $(4,12)$. The main benefit of this treatment is the longterm outcome. The cartilage responds naturally to trauma and no changes in the cartilage over time are observed. Long-term complications are minimal, leading to lower costs and burden for the patient compared to other techniques.

The surgical technique of ear reconstruction with rib grafts is technically challenging with a steep learning curve. Treatment by specialised surgeons who perform this type of surgery on a regular basis is preferred to achieve the best results. Other drawbacks of this surgery include the burden for the patient, which is higher compared to other treatments due to harvesting the rib graft $(4,5)$. 


\section{- Outcome importance}

The choice for one of the treatment options for ear reconstruction is based on the potential outcomes, benefits and harms of treatment. Patients and parents should be able to make their choice based on the available information and weigh the advantages and disadvantages of treatment options and should be supported if they decide that no reconstruction is the correct option for them. The conclusions and recommendations in this chapter aim to help for patients and doctors in the process of choosing a type of treatment.

\section{- Costs and resources}

The impact on costs and resources of the given recommendations will vary per member state, depending on the available care providers and facilities. Treatment with porous polyethylene prosthesis (Medpore) could contribute to an increase in costs. Health insurance reimbursement and the willingness to cover these costs may differ between countries. Costs are lowest and resources are most efficiently used when care for craniofacial disorders is centralised in a limited number of expert centres per member state. A general rule that can be applied is one expert centre per 10 million inhabitants.

\section{- Inequity of the recommendation}

The goal of the European Reference Networks (ERNs) is to eliminate inequality within Europe with regard to care for patients with rare diseases. At present, not every member state offers an expert centre for CFM, or the level of provided care does not (yet) meet all the requirements that are outlined in this guideline. By defining the baseline of required care for CFM, this guideline will help these member states to reach the appropriate level. The ERN on craniofacial anomalies and ENT disorders (ERN-CRANIO) can guide a patient in Europe to the available centres of expertise (www.ern-cranio.eu) and can support care providers with diagnosis and treatment advice.

\section{- Feasibility of the recommendation}

Recommendations refer to the general requirements for delivering optimal healthcare and are discussed with members from participating European countries. Quality of care was paramount in the discussions. Centralisation is proposed as one of the core values. However, in some countries the national organisation of healthcare might impede centralisation. National implementation of the ERNs that fits the situation of each country is necessary. For the member states with the lowest number of inhabitants, the establishment of a craniofacial centre might not be feasible, and collaboration with a craniofacial centre in the surrounding countries can be an option.

\section{- Acceptability of the recommendation}

It is expected that all stakeholders want to apply and will be applying the recommendations, because they are employed in ERNacknowledged institutions. National implementation plans are necessary to ensure that recommendations fit the situation in each country. In addition, not all countries participating in the ERNCRANIO are represented in the guideline development group, and new members will join within the coming years. For these countries, acceptance and implementation of ERN guidelines such as this guideline on CFM is obligatory.

\section{Rationale of the recommendations}

There is no strongly preferred treatment option to treat microtia in patients with CFM. The selected treatment should be based on patients' preferences, which is only possible if the surgeon informs the patient (and parents) on all the advantages and disadvantages of the various options. Autologous reconstruction with rib grafts is considered the most durable treatment options with good long-term outcomes. This is especially relevant in patients with congenital microtia, since these patients need a life-long treatment solution. The timing of treatment depends on the psychosocial development of the child, the availability of cartilage for reconstruction with rib, the size of the natural ear, and the ability of the child to be part of the decision-making process and to cooperate with the surgical management. Ear reconstruction with an autologous rib graft can be performed from the age of eight since by then enough cartilage can be harvested to reconstruct the ear. It is advised to place Medpore implants after the age of six, because by then, the width of the ear approaches its mature size (22). Treatment with external silicone prosthesis attached with adhesives may be initiated in children. However, treatment at a young age is discouraged since this emphasises that the child is 'different from normal'. Treatment of the ear deformity at a young age is therefore often done to comfort the parents, which makes the indication for treatment debatable. Their worries about their child being bullied should be taken seriously and support should be provided, but surgery will not be the solution. Psychosocial support by a psychologist prior to and after treatment is important, especially in patients with aesthetic concerns. Given all the advantages and disadvantages of all options, ear reconstruction with rib grafts is considered to be the gold standard. An external prosthesis on osseointegrated implants is ideally used as a secondary option for salvage cases. Treatment should be discussed within the multidisciplinary team to ensure optimal timing in the planning of other surgeries. Figure 1 shows a flowchart of the recommendations on the various treatment modalities that can be performed to treat microtia in patients with CFM.

\section{Recommendations}

- Patients should be treated within a multidisciplinary team setting.

- Discuss the advantages and disadvantages of the various treatment modalities with the patient and base the choice for treatment on patients' preferences.

- Psychological input is required pre- and postoperatively to monitor expectation and acceptance.

- Ear reconstruction with rib grafts is the first choice of treatment.

- Perform ear reconstruction with rib from the age of eight onwards.

- Treatment before the age of eight is not recommended, but if chosen, use external silicone prosthesis attached with adhesives.

- If chosen, place polyethylene implants (Medpore) from the age of six onwards.

- Osseointegrated implants are an option for salvage procedures.

- Outcome measures should be obtained pre- and postoperatively with all techniques and interventions.

\section{Research gap}

Measurement of an agreed set of outcomes should be done before and after surgery in order to assess and compare the benefit of the procedure to allow benchmarking. 
Figure 3. Flowchart of recommendations for treatment of microtia in craniofacial microsomia

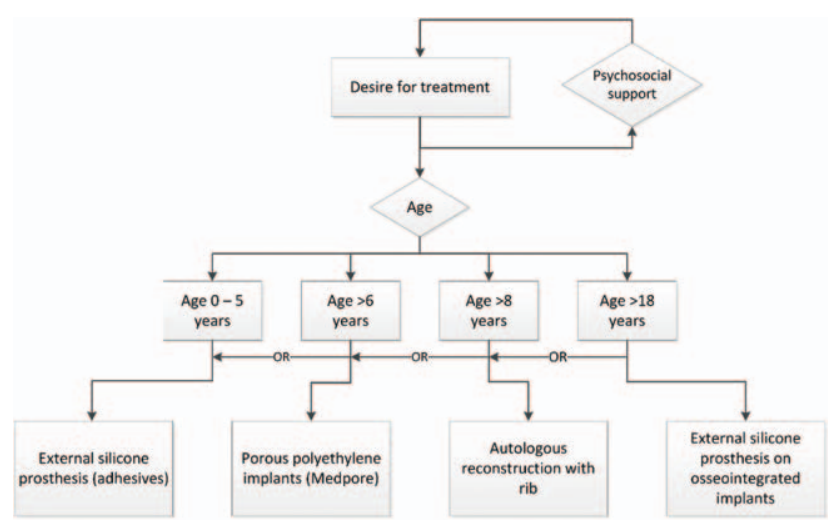

\section{References}

1. Caron C, Pluijmers BI, Wolvius EB, Looman CWN, Bulstrode $\mathrm{N}$, Evans RD, et al. Craniofacial and extracraniofacial anomalies in craniofacial microsomia: a multicenter study of 755 patients'. J Craniomaxillofac Surg. 2017;45(8): 1302-10.

2. Tuin J, Tahiri Y, Paliga JT, Taylor JA, Bartlett SP. Distinguishing Goldenhar Syndrome from Craniofacial Microsomia. J Craniofac Surg. 2015;26(6):1887-92.

3. Birgfeld CB, Heike C. Craniofacial microsomia. Semin Plast Surg. 2012;26(2):91-104.

4. UK Care Standards for the Management of Patients with Microtia and Atresia.

5. Mandelbaum RS, Volpicelli EJ, Martins DB, Park SH, Dubina E, Ishiyama A, et al. Evaluation of 4 Outcomes Measures in Microtia Treatment: Exposures, Infections, Aesthetics, and Psychosocial Ramifications. Plast Reconstr Surg Glob Open. 2017;5(9):e1460.

6. Park JY, Park C. Microtia Reconstruction in Hemifacial Microsomia Patients: Three Framework Coverage Techniques. Plast Reconstr Surg. 2018;142(6):1558-70.

7. Qian J, Li Z, Liu T, Zhou X, Zhang Q. Auricular Reconstruction in Hemifacial Microsomia with an Expanded Two-Flap Method. Plast Reconstr Surg. 2017;139(5):1200-9.

8. Li Y, Zhang R, Li D. A Systematic Review of Levels of Evidence in Auricular Defects Literature: How Far Has It Been During the Past Three Decades? J Craniofac Surg. 2015;26(7):2062-6.

9. Long X, Yu N, Huang J, Wang X. Complication rate of autologous cartilage microtia reconstruction: a systematic review. Plast Reconstr Surg Glob Open. 2013;1(7):e57.

10. Younis I, Gault D, Sabbagh W, Kang NV. Patient satisfaction and aesthetic outcomes after ear reconstruction with a Branemark-type, bone-anchored, ear prosthesis: a 16 year review. J Plast Reconstr Aesthet Surg. 2010;63(10):1650-5.

11. Braun T, Gratza S, Becker S, Schwentner I, Stelter K, Patscheider M, et al. Auricular reconstruction with porous polyethylene frameworks: outcome and patient benefit in 65 children and adults. Plast Reconstr Surg. 2010;126(4): 1201-12.

12. Constantine KK, Gilmore J, Lee K, Leach J, Jr. Comparison of microtia reconstruction outcomes using rib cartilage vs porous polyethylene implant. JAMA Facial Plast Surg. 2014;16(4): 240-4.
13. Fu YY, Li CL, Zhang JL, Zhang TY. Autologous cartilage microtia reconstruction: Complications and risk factors. Int $\mathbf{J}$ Pediatr Otorhinolaryngol. 2019;116:1-6.

14. Roos EW, S.O. Öberg, M. Growth of ears reconstructed from autologous rib cartilage. Eur J Plast Surg. 2015(38):443-8.

15. Al Kadah B, Naumann A, Schneider M, Schick B, Linxweiler M, Papaspyrou G. Auricular reconstruction with polyethylene implants or silicone prosthesis: A single institution experience. J Craniomaxillofac Surg. 2018;46 (12):2150-6.

16. Si Y, Fan SC, Sun W, Chen YB, Zhang ZG. Osseointegration technique in patients with acquired auricular deformities and failed previous reconstruction: a retrospective study of longterm follow-up and Chinese experience. ORL J Otorhinolaryngol Relat Spec. 2012;74(3):129-35.

17. Vijverberg MA, Verhamme L, van de Pol P, Kunst HPM, Mylanus EAM, Hol MKS. Auricular prostheses attached to osseointegrated implants: multidisciplinary work-up and clinical evaluation. Eur Arch Otorhinolaryngol. 2019;276 (4):1017-27.

18. Wright RF, Zemnick C, Wazen JJ, Asher E. Osseointegrated implants and auricular defects: a case series study. J Prosthodont. 2008;17(6):468-75.

19. Li D, Chin W, Wu J, Zhang Q, Xu F, Xu Z, et al. Psychosocial outcomes among microtia patients of different ages and genders before ear reconstruction. Aesthetic Plast Surg. 2010;34(5):570-6.

20. Akter F, Mennie JC, Stewart K, Bulstrode N. Patient reported outcome measures in microtia surgery. J Plast Reconstr Aesthet Surg. 2017;70(3):416-24.

21. Soukup B, Mashhadi SA, Bulstrode NW. Health-related quality-of-life assessment and surgical outcomes for auricular reconstruction using autologous costal cartilage. Plast Reconstr Surg. 2012;129(3):632-40.

22. Farkas LG, Posnick JC, Hreczko TM. Anthropometric growth study of the ear. Cleft Palate Craniofac J. 1992;29(4):324-9.

\section{CHAPTER 6. ORGANISATION OF CARE}

\section{Minimal care standards and monitoring outcomes}

\section{Introduction}

Craniofacial microsomia (CFM) is a condition involving various congenital disorders which requires the involvement of multiple healthcare professionals. A multidisciplinary team is needed to provide and align the complex, long-lasting care for patients with CFM.

Since CFM is a relatively rare congenital disorder, with an incidence of 1:3,000 to 1:5,000 live births, centralisation of care in specialised craniofacial centres is preferred since this makes it possible to build experience in treating these patients, improve the quality of care, and to perform clinically relevant scientific research.

Multidisciplinary care requires good coordination and communication among healthcare professionals, but also with patients and parents of patients. The responsibility and division of tasks of all professionals should be clear for all team members. Centralisation of care and formation of multidisciplinary craniofacial teams makes it possible to perform comparative studies between centres. Comparing outcomes of the various craniofacial centres would have a positive effect on the quality of care.

To make recommendations on the minimal care standards for the treatment of patients with CFM, the following question was posed: 
3.1 What are the minimal care standards to treat patients with craniofacial microsomia and how should outcomes of care be monitored?

Defining the minimal care standards to treat patients with CFM is important to guarantee good quality of treatment for all patients with CFM. Patients of the hospitals participating in the ERNCRANIO were asked to identify current problems in the care process (patient bottlenecks). Patients reported problems in getting referred to a specialised centre, the lack of a multidisciplinary approach and long travel distance. Additionally, patients advised appointing a care provider as point of contact within the craniofacial team, who can be contacted by patients, parents of patients and healthcare professionals.

Literature search

A systematic search of literature was performed to identify all available literature on CFM and synonyms. The search was conducted in Embase, Pubmed/Medline Ovid. The full search strategy is reported in the supplementary material.

Inclusion and exclusion criteria:

Type of studies

- Original articles

- Systematic review of sufficient quality:

- The question in the systematic review

matches the question of the guideline.

- The search of the systematic review was conducted in at least two relevant

databases, such as the Cochrane Library, Medline/Pubmed.

- The full search strategy was reported.

- No relevant items were missing in the search strategy.

Type of patients $\quad$ - Patients with craniofacial microsomia Subject

- Organisation of care

- Patient reported bottleneck in care process - Outcome measures

Exclusion criteria

- Original studies with $<10$ included patients

- Articles published before 1980

- Case reports

- Expert opinion

- Letters

- Editorials

- Narrative reviews

Literature was screened on title and abstract. One article, by Johns et al., was included which discussed the experiences of patients with CFM during medical care (1).

6.1 What are the minimal care standards to treat patients with craniofacial microsomia and how should outcomes of care be monitored?

The literature search for this chapter was directed towards bottlenecks mentioned by patients regarding communication, information, referral, and collaboration. Results of the bottleneck analysis that was performed by the authors of this guideline are displayed in the appendix. The review of literature and conclusions will be based on the article of Johns et al (1). The considerations and recommendations in this chapter will be based on both expert opinion, patient preferences and literature.

\section{Review of literature}

Johns et al. approached patients with CFM and caregivers of patients with CFM from the USA to share their views and experiences regarding their situation at home, school, community and medical care. A total of 42 caregivers and 9 adult patients were included. When asked about the difficulties in medical care, $25 \%$ of the caregivers reported a lack of knowledge about CFM among healthcare professionals along the whole healthcare pathway. Additionally, $16 \%$ of the caregivers and $20 \%$ of the patients reported that there was little guidance during treatment. The coordination of care was troublesome for some patients: $13 \%$ of the caregivers reported difficulties accessing treatment; unfortunately it was not clarified what the reasons for these difficulties were (e.g. organisational or insurance problems). Another $13 \%$ of the patients received conflicting medical recommendations, and $9 \%$ found it difficult to coordinate care with the multiple healthcare providers.

A third of the caregivers and half of the adult patients suggested improving treatment communication and coordination during the care process. Caregivers recommended providing clear information about the diagnosis CFM (16\%) and stated they would like to be more informed about CFM (13\%). Appropriate referral to specialist care $(13 \%)$, parent support $(10 \%)$, help with insurance coverage $(6 \%)$ and support of patient organisations (17\%) were other issues that caregivers and patients mentioned. A patient-centred approach and involving the family in decision making regarding treatment was recommended by the patients.

\section{Conclusions}

Level 3

The most frequently reported difficulties in care for patients and parents of patients with craniofacial microsomia include healthcare professionals' lack of knowledge regarding the diagnosis craniofacial microsomia, lack of guidance during treatment and unclear coordination of care. $\operatorname{Ref}(1)$

Level 3

Communication about and coordination of the treatment
during the care process should be improved to
optimise treatment for patients with craniofacial
microsomia. A patient-centred approach and
involvement of the family is recommended by
patients.
Ref (1)

\section{Considerations}

- Overall quality of evidence

The quality of evidence of the conclusions was graded as level 3 since the study by Johns et al. is a cross-sectional, noncomparable study.

\section{- Balance of benefits and harms}

Information

Patients mentioned that they would prefer to have more knowledge about the condition, about possible treatments, and about specialised professionals and institutions in their own or neighbouring country. Information about CFM is scarce in most countries. In addition, patients mentioned that they were forced to find their own information on the internet and in books. CFM might result in lifelong medical treatment or disability of the patient. Information is rarely available throughout the healthcare process. Similarly, doctors in urban hospitals are not always aware where CFM is best treated in their country. Clear protocols and patient information are necessary. 
Developing information for patients, parents and professionals takes effort, but it will eventually help both patients, parents and professionals. This guideline will point out gaps in knowledge on which further research can focus.

Communication

Patients mentioned that different teams in various hospitals might come up with different treatment options. This is quite difficult for patients and parents to understand. Patients prefer to have a contact person each centre; a care coordinator who will function as a point of reference for the patient, parents and professionals and will clarify and facilitate communication between different institutions and within her/his own institution. This role could be played, for example, by a (specialist) nurse.

Communication between and within teams is necessary to facilitate the best possible treatment and help patients and parents in the best way possible. This might require an extra effort from the team but it will make communication for parents, patients and professionals much better.

\section{Referral}

Patients mentioned that they were often referred after many months. In addition, there was unclearness about the city or country of specialists. Timely referral to the correct centre of expertise facilitates the whole healthcare process for patients and parents. Optimal referral will result in less overtreatment, receiving the wrong treatment, and less waiting time for the patient and the professional. It will make the healthcare path smoother and will result in less unnecessary healthcare.

Collaboration and conditions

Patients mentioned that it sometimes felt as if people from different disciplines worked individually instead of functioning as a multidisciplinary team. Specialists often worked in different hospitals, which would not be an issue if optimal communication could be arranged. Multidisciplinary care requires good coordination and communication within the team itself, towards practitioners involved outside the center and with the patient and parents. This can be difficult. The responsibility and division of tasks for the various care providers within the team should therefore be clearly described. This is a responsibility of the centre of expertise.

Treating patients with CFM requires input from several experts; therefore, coordination of healthcare is important. A multidisciplinary team should meet the following requirements:

1. Composition of and collaboration within a multidisciplinary team

2. Experience with treatment of CFM

3. Access to the necessary facilities:
a. (3D)photography, roentgen, CT, MRI, 3D-planning facility
b. Paediatric ICU
c. Sleep study facility
d. Audiological evaluation
e. Dental lab
Good accessibility of care

4.

5. Core team working in one facility:
a. Maxillofacial surgeon
b. Plastic surgeon
c. ENT/audiology
d. Psychology
e. Orthodontics
f. Ophthalmologist
g. Paediatric anaesthesiologists
h. Team coordinator

Access to a wider team (even outside the hospital):

6. a. Paediatrician

b. Clinical geneticist

c. Paediatric intensivist

d. Neurosurgeon and/or orthopaedic surgeon for spinal anomalies

e. Paediatric radiologist

f. Social worker

g. Speech therapists

h. Pedagogical worker

i. (Facial) physical therapist

j. Prosthetist

k. Respiratory team

Continuity of care for patients who reach adulthood 7. (transitional care)

8. Systematic evaluation of outcomes and implementing changes that are the result of these evaluations

9. Innovation and scientific research (congresses and publications)

10. Additional and continuing training of all team members

11. Updated information for patients and caregivers

\section{Centralisation}

Healthcare for patients with CFM requires a multidisciplinary approach, given the complex care these patients need. Centralisation results in more expertise, higher quality of care and more possibilities for scientific research to improve care.

Healthcare for patients with CFM is centralised in expertise centres in different countries in Europe. However, patients are not always aware of this. In addition, not every European country will have an specialist centre for CFM. Centralisation might result in less expertise being present in other institutions and a longer travel distance for patients. Because a large part of the healthcare for CFM concerns elective care this rarely leads to medically undesirable situations. Centralisation is the only way to achieve good evaluation of new treatment methods and comparative studies.

Professionals treating CFM are already connected in European Reference Networks (ERNs) with a focus on complex craniofacial anomalies and ear, nose and throat (ENT) disorders (ERN-CRANIO).

European Reference Networks (ERNs) are virtual networks of healthcare providers from across Europe. The networks aim to pool together expertise on complex and rare diseases and concentrate knowledge and resources. There are 24 ERNs, each focusing on a particular disease area. A general rule of thumb is that one expertise centre is required for a specific group of diagnoses for every 10 million inhabitants.

ERN-CRANIO seeks to facilitate cooperation between multidisciplinary experts across Europe to support the provision of high-quality care. Initially there are 29 European hospitals involved in ERN-CRANIO, from 11 member states. It is a multidisciplinary network of highly specialised healthcare professionals. Further information can be found on the website https://ern-cranio.eu.

By encouraging cooperation between countries, patients in need of specialised treatment or patients with rare diseases are offered the possibility to choose from a wider range of healthcare providers and to access alternative or specialised treatment abroad more easily.

Monitoring

Current practice varies in different European countries. In addition to developing a guideline with standards of care, it is relevant to monitor the outcome of these standards of care in the 
future with the participating institutions. Monitoring standards of care should encourage institutions to change their current practice if results of treatment are falling short. In addition, institutions can learn from each other and can help each other by implementing optimal organisation of care. It is important to mention that monitoring standards of care will not be set up to criticise each other but to improve the quality of healthcare together.

Monitoring standards of care might require some effort from the team but can result in many valuable (international) results that will improve quality of care.

\section{- Outcome importance}

Patients mentioned in the bottleneck analysis that they prefer a care coordinator, clear referral, a multidisciplinary approach, more cooperation between several specialists, and more knowledge about the disease, about possible treatments, and about specialised professionals and institutions in their own neighbouring country.

\section{- Costs and resources}

The impact on costs and resources of the given recommendations will vary per member state, depending on the available care providers and facilities. The recommendations concern the essential requirements for adequate treatment of patients with CFM and should thus be implemented. Costs are lowest and resources are most efficiently used when care for craniofacial disorders is centralised in a limited number of expert centres per member state. A general rule that can be applied is one expert centre per 10 million inhabitants.

\section{- Inequity of the recommendation}

The goal of the European Reference Networks (ERNs) is to eliminate inequality within Europe with regard to care for patients with rare diseases. At present, not every member state offers an expert centre for CFM, or the level of provided care does not (yet) meet all the requirements that are outlined in this guideline. By defining the baseline of required care for CFM, this guideline will help these member states to reach the appropriate level. The ERN on craniofacial anomalies and ENT disorders (ERN-CRANIO) can guide a patient in Europe to the available centres of expertise (www.ern-cranio.eu) and can support care providers with diagnosis and treatment advice.

\section{- Feasibility of the recommendation}

Recommendations refer to the general requirements for delivering optimal healthcare and are discussed with members from participating European countries. Quality of care was paramount in the discussions. Centralisation is proposed as one of the core values. However, in some countries the national organisation of healthcare might impede centralisation. National implementation of the ERNs that fits the situation of each country is necessary. For the member states with the lowest number of inhabitants, the establishment of a craniofacial centre might not be feasible, and collaboration with a craniofacial centre in the surrounding countries can be an option.

\section{- Acceptability of the recommendation}

It is expected that all stakeholders want to apply and will be applying the recommendations, because they are employed in ERN-acknowledged institutions. National implementation plans are necessary to ensure that recommendations fit the situation in each country. In addition, not all countries participating in the
ERN-CRANIO are represented in the guideline development group, and new members will join within the coming years. For these countries, acceptance and implementation of ERN guidelines such as this guideline on CFM is obligatory.

\section{Rationale of the recommendations}

The guiding principle of the recommendations is to offer the most optimal care to children with CFM and their parents. The assessment and treatment of this condition is complex and has a lifelong impact on the patients. This necessitates a dedication from healthcare providers to continue training in all aspects of this care.

\section{Information}

- The multidisciplinary team should provide information regarding the condition and treatment options based on the present craniofacial microsomia guideline in their own language.

\section{Recommendations}

\section{Referral}

- Patients should be referred to the multidisciplinary craniofacial microsomia team in a timely manner.

\section{Collaboration}

- Care for patients with craniofacial microsomia should be delivered by the multidisciplinary team.

- The clinical pathway based on this guideline should be followed.

\section{Communication}

- Communication between and within teams (also in different hospitals) should be initiated to facilitate the best possible treatment. A contact person in each centre - a care coordinator - clarify and facilitate communication between different institutions and within her/his own institution.

\section{Conditions}

- A craniofacial centre has the following care providers:

- Maxillofacial surgeon

- Plastic surgeon

- ENT/audiology

- Psychology

- Orthodontics

- Ophthalmologist

- Paediatric anaesthesiologists

- Team coordinator 
- Paediatrician

- Clinical geneticist

Paediatric intensivist

- Neurosurgeon and/or orthopaedic surgeon for spinal anomalies

- Paediatric radiologist

- Social worker

- Speech therapists

- Pedagogical worker

(Facial) physical therapist

Prosthetist

- Respiratory team

A craniofacial centre has access to the following care - facilities:

○ (3D)photography, roentgen, CT, MRI, 3D-planning facility

- Paediatric ICU

- Sleep study facility

- Audiological evaluation

- Dental lab

\section{Transitional care}

- Continuity of care should be ensured for patients with craniofacial microsomia who reach adulthood.

\section{Centralisation}

- Patients with craniofacial microsomia are only treated for craniofacial microsomia-related difficulties in a centres that meets the criteria (including volume of care) defined by the ERN-CRANIO.

\section{Monitoring}

- Patient measure should be performed as stated in each chapter.

- Adhere to the ERN-CRANIO registry.

\section{Research gap}

Research can be directed towards the following gaps: distribution of information on CFM for patients, parents and professionals; optimising referral to specialised care centres; optimising coordination and communication between specialist within and between different hospitals; and optimising the role of psychosocial care for patients with CFM. All patient reported outcome measures should be studied for patients with CFM and be translated and validated in all ERN-CRANIO languages.

\section{References}

1. Johns AL, Luquetti DV, Brajcich MR, Heike CL, Stock NM. In Their Own Words: Caregiver and Patient Perspectives on Stressors, Resources, and Recommendations in Craniofacial Microsomia Care. J Craniofac Surg 2018;29:2198-205. 